\title{
Evaluierung des Förderschwerpunkts Talente
}

Endbericht

Wien, Dezember 2020

www.kmuforschung.ac.at 
Diese Studie wurde im Auftrag des Bundesministeriums für Klimaschutz, Umwelt, Energie, Mobilität, Innovation und Technologie (BMK) durchgeführt.

\section{$\mathcal{L}$ KMU Forschung Austria}

Verfasserlnnen der Studie
Andrea Dorr
Eva Heckl (Projektleitung)
Joachim Kaufmann

Die vorliegende Studie wurde nach allen Maßstäben der Sorgfalt erstellt.

Die KMU Forschung Austria übernimmt jedoch keine Haftung für Schäden oder Folgeschäden, die auf diese Studie oder auf mögliche fehlerhafte Angaben zurückgehen.

Für Rückfragen zur Studie

Eva Heckl

Tel.: +43150597 61-36

e.heckl@kmuforschung.ac.at www.kmuforschung.ac.at
Internes Review / Begutachtung

Peter Kaufmann

Dieses Werk ist urheberrechtlich geschützt. Jede Art von Nachdruck, Vervielfältigung, Verbreitung, Wiedergabe, Übersetzung oder Einspeicherung und Verwendung in Datenverarbeitungssystemen, und sei es auch nur auszugsweise, ist nur mit ausdrücklicher Zustimmung des Auftraggebers der Studie / der KMU Forschung Austria gestattet.

Mitglied bei:

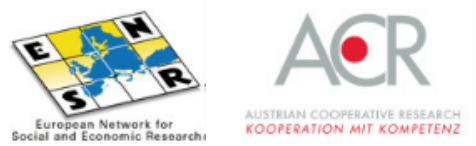




\section{Inhaltsverzeichnis}

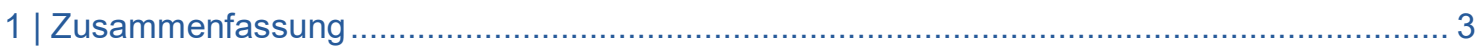

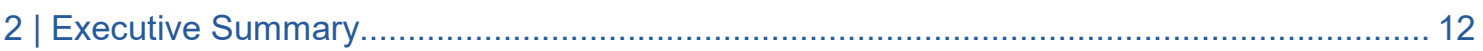

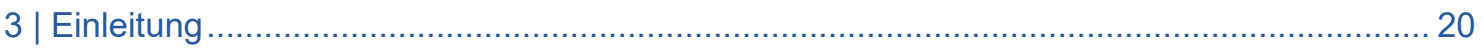

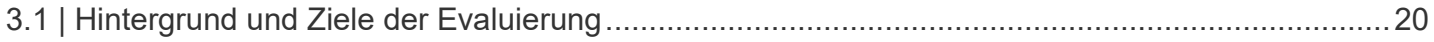

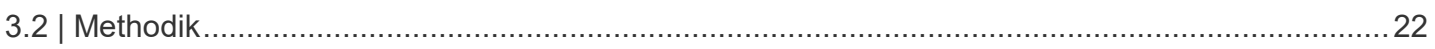

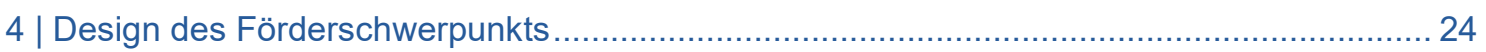

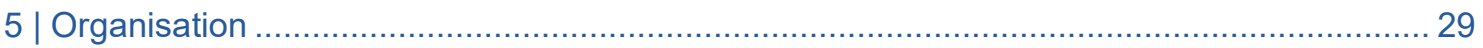

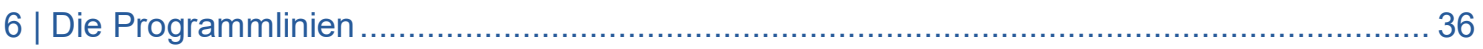

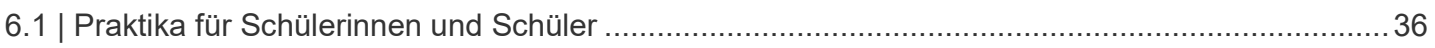

6.1.1 | Struktur der geförderten Praktikumsplätze und Organisationen ........................................37

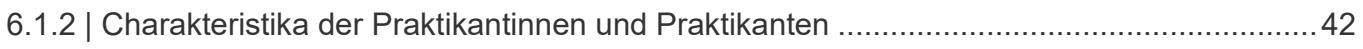

6.1.3 Beurteilung und Wirkungen der Praktika für Schülerinnen und Schüler.............................46

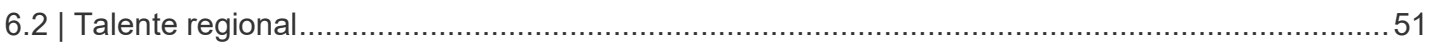

6.2.1 | Struktur der geförderten Talente regional Projekte …….............................................. 52

6.2.2 | Beschreibung der ProjektteilnehmerInnen ……….................................................... 55

6.2.3 | Wirkungen der Talente regional Projekte und Beurteilung der Programmlinie...................58

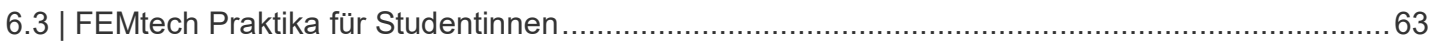

6.3.1 | Struktur der geförderten Praktikumsplätze für Studentinnen............................................63

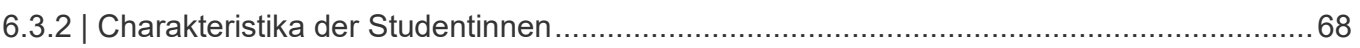

6.3.3 Beurteilung und Wirkungen der FEMtech Praktika für Studentinnen ................................70

6.4 | FEMtech Karriere und FEMtech Karriere-Check für KMU ...................................................76

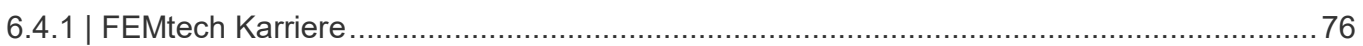

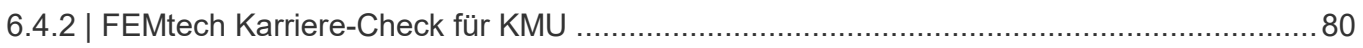

6.4.3 | Projektanalyse FEMtech Karriere und FEMtech Karriere-Check für KMU .........................81

6.4.4 | Ergebnisse und Wirkungen sowie Beurteilung der Programmlinien................................. 85

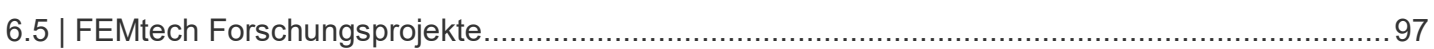

6.5.1 I Struktur der geförderten FEMtech Forschungsprojekte und der Projekt-
teilnehmerlnnen

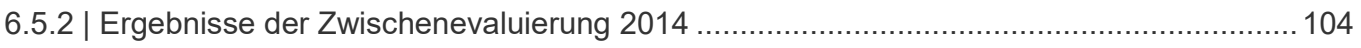

6.5.3 Ergebnisse des Reviews der FEMtech Forschungsprojekte 2016 und abschließende Beurteilung der Programmlinie ..................................................... 104

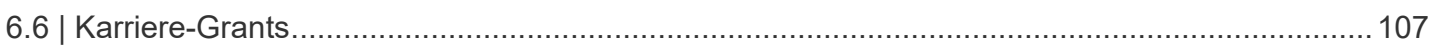

6.6.1 Ansprache der Zielgruppe, Struktur der Einreichungen und vergebene Förderungen ..... 108

6.6.2 | Inanspruchnahme der Grant-Arten durch die FördernehmerInnen ............................. 112

6.6.1 | Wirkungen und Bewertung der Karriere-Grants ............................................. 117

6.7 | Die österreichische Jobbörse für Forschung, Entwicklung und Innovation............................... 121

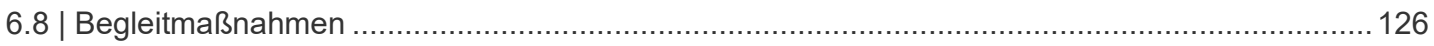




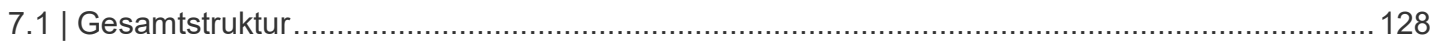

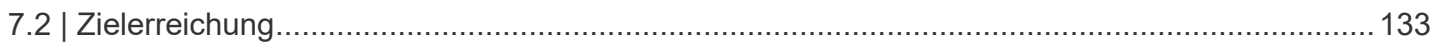

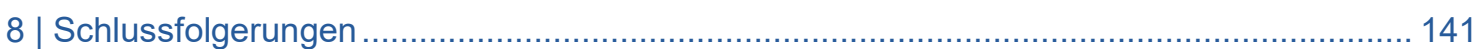

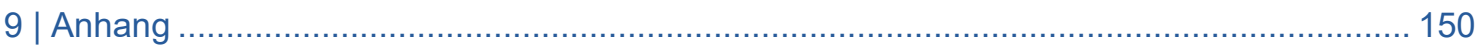

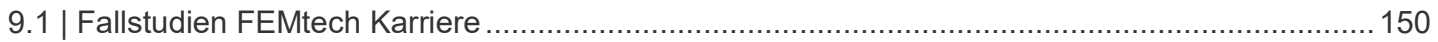

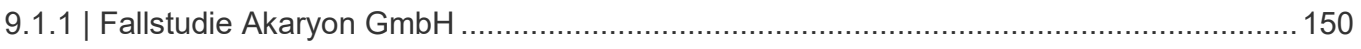

9.1.2 | Fallstudie BEST - Bioenergy and Sustainable Technologies GmbH ............................ 152

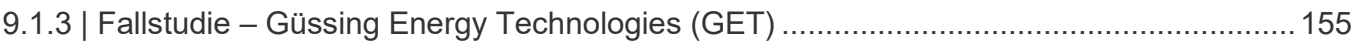

9.1.4 | Fallstudie im-plan-tat Raumplanungs-GmbH \& Co KG .................................................. 158

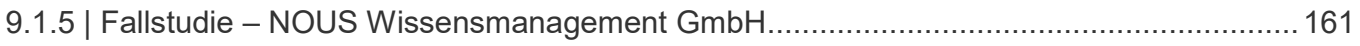

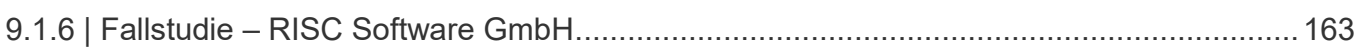

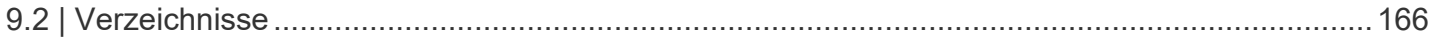




\section{1 | Zusammenfassung}

Mit dem Förderschwerpunkt Talente fördert das Bundesministerium für Klimaschutz, Umwelt, Energie, Mobilität, Innovation und Technologie (BMK) Menschen in der angewandten Forschung über den gesamten Karriereverlauf. Übergeordnetes Ziel ist die erhöhte Ausschöpfung des Humanpotentials im anwendungsorientierten, naturwissenschaftlich-technischen FTI-Bereich. Die drei Zielsetzungen sind

- junge Menschen für Forschung und Entwicklung zu begeistern,

- Forscherinnen und Forscher mit der Wirtschaft zu vernetzen und

- gleiche Chancen für alle zu garantieren.

Im Rahmen dreier Interventionsfelder finden sich verschiedenste Programmlinien:

- Interventionsfeld Talente entdecken: Nachwuchs mit den Programmlinien Praktika für Schülerinnen und Schüler sowie Talente regional

- Interventionsfeld Talente nützen: Chancengleichheit mit den Programmlinien FEMtech Praktika für Studentinnen, FEMtech Karriere und FEMtech Karriere-Check für KMU (2015 und 2016), FEMtech Forschungsprojekte

- Interventionsfeld Talente finden: Forscherinnen und Forscher mit den Programmlinien Karriere-Grants und der Jobbörse

Nach einer Zwischenevaluierung im Jahr 2014 wurde nun am Ende der Programmlaufzeit (Ende 2020) eine Endevaluierung durchgeführt. Im Rahmen der Evaluierung wurde das Programm in Hinblick auf Konzeption, Umsetzung, Zielerreichung und Wirkungen analysiert und darauf aufbauend Schlussfolgerungen und Empfehlungen für die Weiterentwicklung des Förderschwerpunkts Talente formuliert. Methodisch stützt sich die Evaluierung auf einer Dokumentanalyse, Sekundärdatenanalyse (FFG-Monitoringdaten), Interviews mit Expertinnen und Experten, OnlineBefragungen von FördernehmerInnen (FEMtech Karriere / FEMtech Karriere-Check für KMU und Karriere-Grants), Fallstudien (FEMtech Karriere Projekte) und Workshops.

\section{Praktika für Schülerinnen und Schüler}

Im Bereich der Nachwuchsförderung ermöglicht die Programmlinie Praktika für Schülerinnen und Schüler Jugendlichen ab 15 Jahren erste Praxiserfahrungen in der naturwissenschaftlichen und technischen Forschung zu sammeln. Im Zeitraum von 2011 bis 2019 wurden insgesamt mehr als 12.900 Praktika von Schülerinnen und Schülern gefördert. Etwa die Hälfte dieser Praktikumsplätze (52 \%) wurde in Unternehmen und ein Drittel (32 \%) in hochschulischen Einrichtungen zur Verfügung gestellt, wobei diese oftmals nicht nur eine/n PraktikantIn, sondern mehrere PraktikantInnen aufnahmen. Rd. ein Fünftel der Praktika für SchülerInnen konzentriert sich auf 10 AnbieterInnen aus der Wirtschaft, dem universitären Bereich und der Forschung. Von den geförderten Praktika können immer mehr Mädchen profitieren, im Jahr 2019 waren es bereits $49 \%$. Vor allem die Vorgabe zur Forcierung von Schülerlnnen aus nicht-technischen Schulen trug zu einer maßgeblichen Steigerung der Praktikantinnen bei. Dadurch konnte auch die Beteiligung von Schülerlnnen aus den allgemeinbildenden höheren Schulen (AHS) auf 53 \% gesteigert werden. AHS- 
SchülerInnen absolvieren wesentlich häufiger ihre Praktika in Forschungseinrichtungen und hier vor allem in Universitäten, dies wird jedoch als wenig problematisch eingestuft, da hier die Praktika den Anstoß für die Bildungsentscheidung nach der Schule geben können, und weniger einen direkten Einstieg ins Berufsleben darstellen.

Sowohl die Schülerlnnen als auch die teilnehmenden Organisationen können von den positiven Wirkungen des geförderten Praktikumsangebots profitieren. Die Fördernehmerlnnen hatten dadurch die Möglichkeit, jungen Menschen einen Einblick in ihr Berufsfeld zu gewähren, das Interesse der SchülerInnen für naturwissenschaftliche und technische Berufe zu wecken und frühzeitig Kontakte zu potentiellen Arbeitskräften herzustellen. Die Schülerlnnen wiederum konnten sich neues Wissen aneignen, erste Erfahrungen in der Arbeitswelt sammeln und einen Einblick in neue Berufsfelder und Forschungsbereiche gewinnen, wobei vor allem AHS-Schülerlnnen von dieser Möglichkeit begeistert waren. Die SchülerInnen haben im Rahmen der Praktika vorwiegend anspruchsvolle Tätigkeiten ausgeübt und ihr Interesse an naturwissenschaftlich-technischen Berufen ist gestiegen. Die Praktika haben sich somit als wichtige Orientierungshilfe für den weiteren Bildungs- und Berufsweg erwiesen.

Handlungsbedarf wird aus ExpertInnensicht lediglich in Hinblick auf eine verstärkte Informationsarbeit im schulischen Bereich, insbesondere bei den AHS, gesehen. Der derzeitige Multiplikatoreffekt der Schulen ist zurzeit aber gering. Hier besteht also noch ein relativ hohes Potential, das bei erfolgreicher Umsetzung eine entsprechend höhere Dotierung der Programmlinie nach sich ziehen sollte. Kontrovers wird die Tatsache gesehen, dass im Rahmen von Talente Pflichtpraktika der BHS gefördert werden. ExpertInnen halten dies für kontraproduktiv, ebenso wie die Tatsache, HTL-SchülerInnen zu fördern, die ja schon eine Ausbildungsentscheidung in Richtung Technik gemacht haben. Ein Ausschluss aller Pflichtpraktika würde aber auch Schülerlnnen der nichttechnischen BHS treffen, ein Ausschluss der HTL würde zudem dazu führen, dass das Interesse der Unternehmen an den Praktika stark sinkt und ihnen die Möglichkeit nimmt, im Rahmen geförderter Praktika zukünftige MitarbeiterInnen, die direkt nach der Schule einsetzbar sind, kennenzulernen.

\section{Talente regional}

Um mehr junge Menschen für die Forschung zu begeistern, werden im Rahmen von Talente regional Projekte mit mindestens 2 Unternehmenspartnerlnnen, einer $/ \mathrm{m}$ wissenschaftlichen Partnerln und 5 Bildungseinrichtungen vom Kindergarten bis zu höheren Schulen gefördert. Darüber hinaus können 10 weitere Bildungseinrichtungen (durch das Angebot von pauschalen Kooperationszuschüssen in der Höhe von $€ 1$ 1.000) einbezogen und die regionalen Netzwerke erweitert werden. Im Zeitraum von 2011 bis 2019 wurden insgesamt 141 Talente regional Projekte mit Fokus auf Themen im Bereich Energie- und Umwelt, Life Sciences, IT und Mobilität realisiert. Dabei setzten sich die Kinder und Jugendlichen mit spannenden Themen auseinander, experimentierten, forschten und lernten Tätigkeiten in Forschung, Technologie und Innovation kennen. 
Die Talente regional Projekte bewirken in erster Linie, dass der Forschungsgeist und die Begeisterung der Kinder und Jugendlichen für Naturwissenschaft und Technik geweckt werden. Kenntnisse und Interesse für diese Bereiche sind gestiegen und der Zielgruppe wurde ein realitätsnaher Einblick in die Tätigkeit von ForscherInnen und TechnikerInnen ermöglicht. Die beteiligten PädagogInnen profitieren vor allem dadurch, dass sie neue pädagogische Konzepte und innovative Unterrichtsmethoden kennenlernen und auf Ebene der Schulen kommt es zu einer Stärkung des Stellenwerts von Naturwissenschaft und Technik sowie zu Verbesserungen der technischen Infrastruktur. Ein weiterer Effekt der Talente regional Projekte ist, dass nachhaltige regionale Kooperationen zwischen Bildung, Wissenschaft und Wirtschaft entstehen, was bei $72 \%$ der befragten ProjektteilnehmerInnen nach Projektabschluss der Fall war.

Die geforderten Kooperationen bringen jedoch auch einen hohen Organisationsaufwand bei der Projektabwicklung mit sich. Auch der Innovationsanspruch der Programmlinie und die zusätzlichen Schwerpunkte auf Geschlechterausgewogenheit und der Integration von Kindern und Jugendlichen unabhängig ihrer sozialen oder geographischen Herkunft werden als herausfordernd wahrgenommen. Hier könnte eine stärkere Flexibilisierung des Instruments Abhilfe schaffen. Beispiele dafür sind eine noch klarere Fokussierung auf bestimmte Altersstufen (z. B. nur Vorschulund Volksschulkinder, nur Sekundarstufe I) sowie eine Aufweichung des Innovationsanspruchs dahingehend, dass erprobte Projekte in adaptierter Form in einem anderen Setting für eine neuerliche Förderung zugelassen werden. Dies könnte auch der Nachhaltigkeit der Projekte zuträglich sein, da erprobte Konzepte neuerlich zur Anwendung kommen.

Da das Instrument insgesamt als erfolgreich eingestuft werden kann und die Nachfrage äußerst hoch ist, könnten mit einer jährlichen Ausschreibung mit entsprechender Mittelerhöhung und der damit größeren Zahl an geförderten Projekten mehr Bildungseinrichtungen und Kinder/Jugendliche erreicht werden. Damit könnten zum einen mehr Regionen von den positiven Effekten profitieren und zum anderen eine größere Kontinuität bei den Interventionen sichergestellt werden beides förderliche Faktoren in Hinblick auf strukturelle Veränderungen.

\section{FEMtech Praktika für Studentinnen}

FEMtech Praktika für Studentinnen unterstützen gezielt Nachwuchswissenschaftlerinnen dabei, in der angewandten Forschung in Naturwissenschaft und Technik Fuß zu fassen. Die Studentinnen lernen in den bis zu 6 Monaten dauernden Praktika berufliche Ein- und Aufstiegswege kennen und erhalten einen Einblick in die angewandte Forschung. Im Zeitraum von 2011 bis 2019 wurden rd. 3.600 geförderte Praktikumsplätze für Studentinnen genehmigt. Die Mehrheit der geförderten Praktikumsstellen wird in außeruniversitären Forschungseinrichtungen (58 \%) angeboten, $42 \%$ finden in Unternehmen statt. Unternehmen bieten vorwiegend einen Praktikumsplatz für Studentinnen an, während Forschungseinrichtungen oftmals mehrere Studentinnen gleichzeitig als Praktikantinnen aufnehmen.

Sowohl Fördernehmerlnnen als auch Studentinnen können von den geförderten Praktika profitieren. Studentinnen haben die Möglichkeit, in das Berufsfeld hinein zu schnuppern und Berufserfahrungen zu sammeln und gleichzeitig profitieren die Unternehmen / Forschungseinrichtungen, indem sie potentielle Mitarbeiterinnen vorab kennenlernen können. Das Praktikum hat bei rd. drei Viertel der Studentinnen zu einem gestiegenen Interesse an einer Tätigkeit in Forschung, 
Naturwissenschaft und Technik geführt. Zudem erleichtern die Praktika den Berufseinstieg für Studentinnen. Im Jahr 2018 konnte rd. ein Viertel der Studentinnen im Anschluss an die Praktikumszeit in der jeweiligen Organisation weiterbeschäftigt werden. Die geförderten Praktika erleichtern somit den Sprung in die Arbeitswelt und helfen den Studentinnen auch längerfristig im Bereich von Forschung, Naturwissenschaft und Technik beruflich Fuß zu fassen.

Einziger Wermutstropfen im Zusammenhang mit dem Instrument ist die schnelle Ausschöpfung der Fördermittel und die meist vorzeitige Schließung der Ausschreibung. Hier sind in den meisten Fällen Unternehmen benachteiligt, weil Forschungseinrichtungen meist gleich zu Beginn mehrere Förderungsansuchen für Praktikantinnen stellen. Um die Praktikumsstellen in Unternehmen zu forcieren, wird daher eine Deckelung der geförderten Praktikumsstellen pro Organisation vorgeschlagen, sodass mehr Organisationen von der Förderung profitieren können und das Instrument breiter gestreut wird. Zudem könnte eine Ausschreibung in zwei zeitlich unabhängigen Phasen, wie sie im Jahr 2019 einmal erfolgte, zu einer weiteren Streuung bei den FördernehmerInnen führen, womit die Praktika noch besser an den Bedarf der Fördernehmerlnnen angepasst werden könnten.

\section{FEMtech Karriere - FEMtech Karriere-Check für KMU}

Zur Förderung der Chancengleichheit wird die Programmlinie FEMtech Karriere eingesetzt, die Organisationen bei der verstärkten Beschäftigung von Frauen in naturwissenschaftlich-technischen Berufen sowie bei der Schaffung guter und fairer Rahmenbedingungen für alle MitarbeiterInnen unterstützt. In den Jahren 2015 und 2016 wurde darüber hinaus der FEMtech KarriereCheck für KMU angeboten, um dieser Unternehmensgruppe die Durchführung einer Genderanalyse zu ermöglichen und sie auf ein mögliches nachfolgendes FEMtech Karriere Projekt vorzubereiten. Im Zeitraum von 2011 bis 2019 wurden 34 FEMtech Karriere Projekte und 17 FEMtech Karriere-Checks für KMU durchgeführt. Der FEMtech Karriere-Check für KMU bot den Unternehmen einen guten Einstieg in die Thematik, denn die Mehrheit dieser Unternehmen (12 von 17 bzw. $71 \%$ ) reichte im Anschluss ein FEMtech Karriere Projekt erfolgreich ein. Im Rahmen der FEMtech Karriere Projekte (Laufzeit 6 bis 24 Monate) werden in den teilnehmenden Organisationen verschiedene Module aus den Bereichen Genderkompetenz, Chancengleichheit und Frauenförderung erarbeitet. Auch die Steigerung der Attraktivität der Organisation als Arbeitgeberln stellt eine wichtige Zielsetzung der Projekte dar. An den Projekten nehmen tendenziell eher Organisationen teil, die bereits mit der Genderthematik und den Förderungen der FFG vertraut sind.

Die Wirkungen der FEMtech Karriere Projekte zeigen sich vor allem in einer Erhöhung der Genderkompetenz sowie in der gestiegenen Akzeptanz und Awareness für das Thema in den beteiligten Organisationen. Auch die Nominierung von Genderbeauftragten, die in 11 von 17 befragten Organisationen erfolgte, stellt eine langfristige Berücksichtigung der Genderthematik sicher. Weitere beobachtbare Effekte sind eine optimierte Außendarstellung und die Steigerung der Attraktivität als Arbeitgeberln, die Verbesserung der Arbeitsbedingungen und der Work-Life-Balance sowie die erfolgreichen Qualifizierungs- und Karriereplanungsaktivitäten von Frauen. Konkrete Steigerungen der Frauen- und Forscherinnenanteile zeigen sich hingegen nur selten. Das Instrument ist in erster Linie als Maßnahme zur Bewusstseinsbildung zu sehen. 
Allerdings wird das Instrument wenig nachgefragt und die AntragstellerInnen haben meist schon eine erhöhte Sensibilität für die Thematik. Für NeueinsteigerInnen sind die Anforderungen oft zu komplex. Um wirkliche strukturelle Änderungen der Rahmenbedingungen für mehr Chancengleichheit in Naturwissenschaft und Technik zu schaffen, müssten FEMtech Karriere Projekte in wesentlich mehr Unternehmen und Forschungseinrichtungen durchgeführt werden, um eine kritische Masse an „gegenderten“ Organisationen und in weiterer Folge Spillover-Effekte zu erzeugen. Die größte Herausforderung ist daher, mehr Unternehmen und Forschungseinrichtungen für das Instrument zu gewinnen. Dafür müssten breite Mobilisierungsmaßnahmen sorgen, die weit über die „Talente-Klientel“ hinausgehen und die typischen FFG-FördernehmerInnen umfassen. Dies bedeutet aber auch eine entsprechende substantielle Erhöhung der finanziellen Mittel für PR-Maßnahmen und Zielgruppenmobilisierung für ein Instrument, das erst mittel- bis langfristig seine Wirkung entfaltet. Selbst wenn diese Mittel nicht bereitgestellt werden, sollten in jedem Fall Good Practice Beispiele und Erfolgsgeschichten bereits geförderter Projekte allen FFG-Kundlnnen breitenwirksamer zugänglich gemacht werden. Zudem sollten mögliche Synergieeffekte mit der Initiative FEMtech des BMK zur Zielgruppen-Mobilisierung geprüft werden.

Neben allgemeinen Mobilisierungsmaßnahmen schlagen Expertlnnen eine Namensänderung vor. „FEMtech Karriere“ impliziere, dass das Programm nur Frauen helfen soll, Karriere zu machen. Im Fokus des Instruments steht aber die Veränderung der Unternehmenskultur, damit die Organisation sich für die Zukunft rüstet und im Kampf um Fachkräfte einen Wettbewerbsvorteil erzielt, da es faire und gute Arbeitsbedingungen für alle schafft. Der Name müsste dies widerspiegeln. Zudem sollte die Möglichkeit forciert werden, im Rahmen des Projekts einen Gender Equality Plan zu entwickeln, der zunehmend bei (internationalen) Förderprogrammen gefordert werden wird bzw. eine gute Referenz darstellt. Und schließlich ist eine Neueinführung eines Instruments für EinsteigerInnen zu diskutieren, um genderunerfahrene Organisationen an das Thema heranzuführen. Damit können diese die für einen erfolgreichen Antrag für FEMtech Karriere benötigte Genderkompetenz erwerben.

\section{FEMtech Forschungsprojekte}

FEMtech Forschungsprojekte ist sowohl innerhalb Österreichs als auch international gesehen eine einzigartige Programmlinie und genießt innerhalb der Genderforschungscommunity mittlerweile auch über Österreich hinausgehend Ansehen. Das Alleinstellungsmerkmal von FEMtech Forschungsprojekte gegenüber anderen Projektförderungen besteht in der starken Aufwertung der Genderdimension, so werden ausschließlich Projekte mit genderrelevanten Inhalten gefördert. Auf diese Weise trägt die Programmlinie zum Ziel von Talente bei, FTI-Vorhaben mit genderrelevanten Inhalten zu initiieren. Die Programmlinie ist bei den FördernehmerInnen sehr beliebt, die Nachfrage entsprechend hoch: von 2011 bis 2018 wurden nur 23 \% (52 Projekte) der 231 eingereichten Projekte für eine Förderung genehmigt. Die FFG hat auf das Review aus dem Jahr 2016 reagiert, so wurden die Ausschreibungsleitfäden ab der 5. Ausschreibung entsprechend den Empfehlungen überarbeitet. 
Da die Programmlinie den Anspruch erhebt, durch die Einbindung der Genderdimension auch die Qualität von (zu entwickelnden) Produkten zu erhöhen, können die Projektergebnisse und Projektmanagementprozesse in den geförderten Projekten für den Bereich Forschung, Entwicklung und Innovation insgesamt von Interesse sein. Daher kann die aus vorhergehenden Analysen geäußerte Empfehlung einer Forcierung der Disseminationsaktivitäten nochmals wiederholt werden. Um eine breitenwirksamere Vermarktung wirtschaftlich erfolgreicher Projekte und eine Reflexion des Forschungs- und Entwicklungsprozesses sowie strukturelle Veränderungen in Organisationen und der Forschungs- und Entwicklungsarbeit zu bewirken, muss die Dissemination aber über das Programm Talente bzw. die Programmlinie hinausgehen und auch andere Veranstaltungen und thematische Plattformen nützen. Hier wird eine verstärkte Vernetzung mit anderen Programmen in der FFG angeregt, um geeignete Disseminationsmöglichkeiten anzuregen. Eine andere Möglichkeit wäre es, im Rahmen anderer Programme der Forschungsförderung den Bereich Gender stärker zu integrieren, beispielsweise über eine höhere Gewichtung der Genderkriterien im Rahmen von Bewertungsverfahren oder der Reservierung eines Teils der Fördermittel für Projekte mit explizitem Genderfokus.

\section{Karriere-Grants}

Die Programmlinie Karriere-Grants ist insofern einzigartig innerhalb des FFG-Förderportfolios, als dass die Zielgruppe der Förderung EinzelforscherInnen sind. Das Ziel der Förderung besteht darin, mittels finanzieller Unterstützungsleistungen Forscherlnnen aus dem Ausland für den Bereich Forschung, Technologie und Entwicklung in Österreich zu gewinnen. Gefördert werden Kosten für Jobinterviews in Österreich sowie Umzugs- und Integrationskosten für Forscherlnnen sowie deren PartnerInnen. Insgesamt gab es im Beobachtungszeitraum 2.241 Einreichungen, davon wurden 1.903 gefördert. Insgesamt waren bis zur Ausschreibung 201957 \% der geförderten Einreichungen Relocation Grants, 38 \% Interview Grants, und 5 \% Dual Career Grants. Forscher nehmen Karriere-Grants häufiger in Anspruch als Forscherinnen. Der überwiegende Anteil der Personen hielt sich zum Zeitpunkt der Antragstellung in Europa auf.

Insgesamt betrachtet zeigten sich die meisten geförderten EinzelforscherInnen sehr zufrieden mit der Förderung, und auch die über die Jahre gestiegenen Antragszahlen weisen auf eine entsprechende Nachfrage hin. Hinsichtlich der Zielerreichung kann auf Basis der verfügbaren Informationen festgehalten werden, dass die Förderung allerdings eine sehr geringe Wirkung erzielt. Das Antreten einer Stelle in Österreich wird so gut wie gar nicht von den Relocation Grants beeinflusst, für mehr als $90 \%$ der Befragten ist dies nicht der Fall. Am ehesten zeigen noch die Interview Grants Wirkung. So gaben etwa rd. ein Viertel (23\%) der Befragten an, sie wären ohne Interview Grant nicht oder wahrscheinlich nicht für das Jobinterview nach Österreich gekommen. Von diesen hätten allerdings rd. $36 \%$ das Interview ersatzweise online abgehalten.

Daher muss das Instrument grundsätzlich in Frage gestellt werden, zumal die Gewinnung von Forscherlnnen aus dem Ausland primär von der Attraktivität des Forschungsstandorts und den Rahmenbedingungen abhängt und ein finanzieller Beitrag für Interview- oder Umzugskosten keine Rolle spielt. Die Maßnahme einzustellen wird auch vor dem Hintergrund der Überzeichnung anderer erfolgreicher Programmlinien innerhalb von Talente empfohlen. Sollte am Instrument dennoch festgehalten werden, ist eine Einschränkung der Zielgruppe des Relocation Grants auf 
Forscherlnnen in geographisch weiter entfernt liegenden Regionen, ev. gekoppelt mit sozioökonomischen Indikatoren (wie z. B. dem Medianeinkommen von ForscherInnen im Herkunftsland) anzudenken. Im Zuge dessen sollte dann auch die maximale Förderhöhe angepasst, d. h. erhöht bzw. entsprechend gestaffelt werden. Auch beim Dual Career Grant stellt sich die Frage, worauf die Förderung abzielt und ob das Ziel erreicht werden kann, denn für die Entscheidung, mit der Partnerin oder dem Partner umzuziehen, spielt dieser keine Rolle.

\section{Die österreichische Jobbörse für Forschung, Entwicklung und Innovation}

Die Jobbörse ist ein Service, das die Suche nach (hoch-)qualifizierten Fachkräften durch Unternehmen bzw. Forschungseinrichtungen und die Suche nach Stellen im Bereich Forschung-, Technologie und Innovation durch Fachkräfte erleichtern soll. Im Zeitraum von 2013 bis 2018 zählte die Website pro Quartal durchschnittlich rd. 5.300 Besucherlnnen, der angestrebte Zielwert konnte hier somit nicht erreicht werden. Im 4. Quartal 2019 wurde der Zielwert von 6.000 Besucherlnnen erreicht, ebenso wie im 1. Quartal 2020, gefolgt von einem Rückgang im 2. und 3. Quartal 2020 (Corona-Krise). Die Zunahme an Ausschreibungen im Beobachtungszeitraum deutet auf eine gestiegene Nutzung durch Organisationen hin. Im Rahmen dieser Evaluierung konnte auf Basis der verfügbaren Informationen nicht abschließend geklärt werden, ob die Jobbörse sowohl für Suchende als auch Jobanbieterlnnen einen Zusatznutzen bringt, der von anderen Dienstleistungsangeboten nicht bereits abgedeckt wird. Es wird daher empfohlen, mittels Userlnnenbefragung zu eruieren, ob die Jobbörse einen entsprechenden Nutzen entfaltet. Dazu könnten zum einen die registrierten Unternehmen befragt werden, deren Websites regelmäßig nach Ausschreibungen gescreent werden, sowie die Besucherlnnen der Jobbörse nach Nutzung der Seite, beispielsweise mittels einer Onsite-Befragung.

\section{Begleitmaßnahmen}

Im Rahmen des Förderschwerpunkts werden verschiedene Begleitmaßnahmen umgesetzt: Prämierungsveranstaltungen in der Programmlinie SchülerInnenpraktika, Netzwerkveranstaltungen im Rahmen von Talente regional, Infoveranstaltungen für FEMtech Forschungsprojekte. Diese dienen in erster Linie der Mobilisierung der Zielgruppen sowie der Sichtbarmachung und Dissemination der Aktivitäten. Zudem gibt es die FEMtech Netzwerktreffen.

Im Rahmen der Prämierungsveranstaltungen in der Programmlinie SchülerInnenpraktika werden die 20 SchülerInnen mit den besten Praktikum-Reports prämiert. Die Praktikum-Reports könnten - wenn der Veröffentlichung zugestimmt wird - ebenfalls online gestellt werden und zur Informationsstreuung und Kundlnnenakquise genützt werden. Zur Stärkung der Netzwerkveranstaltungen von Talente regional wird empfohlen, die Vorstellung von Good Practice Beispielen und die Möglichkeit, sich mit erfahrenen Fördernehmerlnnen auszutauschen, noch stärker in den Fokus der Veranstaltung zu rücken sowie ein Online-Format anzubieten, um zusätzlichen Organisationen eine Teilnahme zu ermöglichen. Die zwei Infoveranstaltungen für FEMtech Forschungsprojekte 2017 und 2018 hatten regen Zulauf, was für das hohe Informationsbedürfnis der potentiellen Fördernehmerlnnen spricht. Corona-bedingt fand die dritte Infoveranstaltung zur 7. Ausschreibung online statt. Es wird angeregt, ein solches Online-Format weiterhin (zusätzlich) anzubieten, um mehr InteressentInnen die Möglichkeit zur Teilnahme zu geben und mehr FEMtech Forschungsprojekte vorstellen zu können. 
Die FEMtech Netzwerktreffen werden veranstaltet, um über Neuigkeiten aus dem Förderschwerpunkt zu informieren und zeigen aktuelle FEMtech Themen auf. Es gibt die Möglichkeit zum informellen Austausch und zur Förderberatung durch die FFG. An den Treffen nehmen vorwiegend Personen teil, die sich mit Chancengleichheit in Forschung, Naturwissenschaft und Technik beschäftigen. Damit kann keine breite Streuung der Thematik in die Forschungscommunity erwartet werden. Um das Thema Chancengleichheit in der Forschung auf die allgemeine Agenda zu setzen und zur breiteren Mobilisierung von AntragstellerInnen kann über eine Öffnung des Formats, von der Einladungspolitik bis zur Streuung der Dokumentation, versucht werden, den InteressentInnenkreis zu erweitern. Dann könnten die Treffen auch besser zur Dissemination der Information über die Förderformate, insbesondere über FEMtech Karriere, genützt werden.

\section{Gesamtbetrachtung und Fazit}

Das Design des Programms wird seinem Anspruch, den gesamten Karriereverlauf zu unterstützen, gerecht und definiert diesen auch breit, da eine sehr weit gefasste Zielgruppe adressiert wird, die potentielle ForscherInnen und damit alle Kinder und Jugendlichen miteinschließt. Es adressiert neuralgische Phasen in der Karriere (z. B. Berufseinstieg) bzw. versucht durch Interventionen diese zu beeinflussen (Bildungsentscheidungen, Berufswahl). Zudem zielt das Programm auf Strukturveränderungen, die den Boden für eine bessere Ausschöpfung des Humanpotentials im anwendungsorientierten, naturwissenschaftlich-technischen FTI-Bereich aufbereiten sollen. Der Förderschwerpunkt Talente stellt als Humanpotentialprogramm im FFG-Portfolio nicht nur eine Ausnahme dar, sondern verbessert indirekt und langfristig gesehen über die Unterstützung von Forscherlnnen auch die Wirkung anderer Forschungsförderungsprogramme, in denen diese ForscherInnen ihre Talente in unterschiedlichen Projekten einsetzen.

Die Breite des Programms ist ein wichtiges Charakteristikum, birgt aber die Gefahr der Zielüberfrachtung sowie einer mangelnden Fokussierung vor dem Hintergrund begrenzter Ressourcen. Insbesondere das Interventionsfeld "Talente finden“ bedarf aus Sicht des Evaluierungsteams einer kritischen Analyse. So scheinen die Instrumente nur teilweise zur Zielerreichung geeignet und die Wirkungen sind sehr gering bzw. nicht umfassend zu beurteilen. Es wird daher empfohlen, dieses Interventionsfeld hinsichtlich seiner Zielsetzungen und der eingesetzten Instrumente grundlegend zu hinterfragen. Auf Basis der Diskussion sollte entweder das Interventionsfeld neu aufgesetzt oder aber zugunsten der anderen Interventionsfelder aufgegeben werden. Mit dem Fokus auf Nachwuchsförderung und Chancengleichheit könnten die Ressourcen konzentrierter eingesetzt werden, das Programm müsste allerdings Abstriche in Hinblick auf seinen umfassenden Ansatz machen.

Essentiell für ein "nicht-klassisches“ Forschungsförderungsprogramm ist die Mobilisierung der Zielgruppen. Zum einen sollen in hohem Maße FFG-EinsteigerInnen für das Programm gewonnen werden, zum anderen gilt es, typische FFG-Klientel für Instrumente zu gewinnen, die eher mittel- bis langfristige Wirkungen entfalten und oftmals nicht direkt sichtbar sind. Der Informationsstreuung kommt daher große Bedeutung zu. Zur Förderung der Breitenwirksamkeit des Programms sollten daher die Mobilisierungsmaßnahmen ausgeweitet werden. 
Der Breitenwirksamkeit steht auch entgegen, dass das Programm in seiner Gesamtheit und unter dem Namen „Talente“ noch immer nicht als solches bekannt ist, im Gegensatz zu den einzelnen Programmlinien bzw. Interventionsfeldern (z. B. FEMtech), die für viele FördernehmerInnen und für die meisten Expertinnen und Experten ein Begriff sind. Hier steht zur Diskussion, das „Branding“ zu verstärken, wobei die Bezeichnung der Programmlinien den Programmnamen enthalten sollten. Auch ist bei der Namensgebung der Programmlinien darauf Bedacht zu nehmen, dass dieser die Förderung adäquat beschreibt (siehe FEMtech Karriere). Wenn das Programm zur Marke wird und an Bekanntheit gewinnt, könnte dies zur Unterstützung seiner Mission, der Entwicklung von Humanpotential für die angewandte Forschung, beitragen. Das Rebranding des gesamten Programms, durch das die Zugehörigkeit aller Programmlinien zum Programm deutlich sichtbar wird, sollte durch einen öffentlichkeitswirksamen Relaunch begleitet werden.

Abschließend muss festgehalten werden, dass ein Programm wie Talente, dass an den Strukturen und Rahmenbedingungen ansetzt und nichts weniger als Veränderungen von tradierten Einstellungen und Rollen bewirken will, bei begrenzten Mitteln immer nur einen kleinen Beitrag leisten kann. Die Wichtigkeit der Förderung des Humanpotentials muss auch innerhalb der Forschungscommunity wirklich „ankommen“ und mehr als ein Lippenbekenntnis darstellen. Denn die Zahlen sind v. a. in Hinblick auf die Repräsentanz von Frauen im Forschungssektor - und hier insbesondere im industriellen Sektor - nach wie vor ernüchternd. Daher bedarf es einer Aufwertung und eines Mainstreaming des Themas innerhalb der FFG und des BMK. Die Themen Humanressourcen und Chancengleichheit müssen als Querschnittsthemen begriffen werden und entsprechende Berücksichtigung in den anderen Programmen finden sowie deren Informationskanäle auch für das Programm Talente genützt werden. 


\section{2 | Executive Summary}

With the funding programme Talents, the Federal Ministry for Climate Action, Environment, Energy, Mobility, Innovation and Technology (BMK) supports people in applied research throughout their entire career. The overarching goal is to increase the utilisation of human potential in the application-oriented, scientific and technical RTI sector. The programme objectives are

- to inspire young people for research and development,

- to connect researchers with the economic sector,

- to guarantee equal opportunities for all.

Within the framework of three fields of intervention, there are various programme lines:

- Intervention field Young Talents with the programme lines Internships for Students and Talents Regional,

- Intervention field Female Talents with the programme lines FEMtech Internships for Female Students, FEMtech Career and FEMtech Career Check for SMEs (2015 and 2016), as well as FEMtech Research Projects,

- Intervention field Professional Talents with the programme lines The Austrian Job Exchange for Research, Development and Innovation as well as Career Grants for Interviews, Relocation and Dual Careers in Applied Research.

After an interim evaluation in 2014, a final evaluation took place at the end of the programme period (end of 2020). The programme was analysed with regard to its conception, implementation, achievement of objectives and impact. Furthermore, conclusions and recommendations for the further development of the Talents programme have been drawn. The methodological basis of the evaluation is a document analysis, secondary data analysis (FFG monitoring data), interviews with experts, online surveys of funding recipients (FEMtech Career / FEMtech Career Check for SMEs and Career Grants), case studies (FEMtech Career projects) and workshops.

\section{Internships for Students}

The programme line Internships for Students - Four Weeks in Science and Technology enables young people aged 15 and older to gain initial practical experience in research in the fields of science and technology. In the period from 2011 to 2019, more than 12,900 internships for students were funded. About half of these internships (52\%) were provided in companies and a third $(32 \%)$ in higher education institutions, which often hosted not just one but several internships. About one fifth of the internships for students are concentrated in 10 organisations from the business sector as well as the university and research sector. More and more girls benefit from the funded internships; in 2019, the share of female participants was already $49 \%$. The requirement to push the participation of students from non-technical schools contributed particularly to a significant increase in the number of female interns. As a result, the participation of students from general secondary schools (AHS) also increased to $53 \%$. AHS students complete their internships much more frequently in research institutions, especially in universities. This is not considered to be a problem, as the internships can provide the impetus for the educational decision after school, and do not represent a direct entry into professional life. 
Both, the students and the participating organisations can benefit from the positive effects of the funded internships. The beneficiaries had the opportunity to give young people an insight into their professional field, to raise the students' interest in professions in the fields of science and technology and to establish contacts with potential employees at an early stage. The students, in turn, were able to acquire new knowledge, gain initial experience in a working environment and gain insight into new occupational fields and research areas. AHS pupils were particularly enthusiastic about this latter opportunity. During the internships, the pupils mainly performed demanding activities and their interest in scientific and technical professions increased. The internships have thus proven to be an important orientation aid for the further educational and career path.

From the interviewed experts' point of view, there is only a need for action with regard to intensified information work in schools, especially in the AHS. However, the current multiplier effect of schools is currently low. There is therefore still a relatively high potential here, which, if successfully implemented, should result in a correspondingly higher funding of the programme line. The fact that compulsory internships at BHS (vocational colleges) are funded within the framework of Talents is seen as controversial. Experts consider this as counterproductive as well the fact that HTL (technical vocational college) students are funded who have already made a decision in the direction of a technical education. An exclusion of all compulsory internships would also affect students of non-technical BHS, and an exclusion of HTL would lead to a strong decrease in the interest of companies in internships and deprive them of the opportunity to get to know future employees who can be employed directly after school.

\section{Talents Regional}

In order to raise the interest of more young people in research, projects with at least two companies, one scientific partner and five educational institutions from kindergarten to secondary schools are funded within the framework of Talents Regional. In addition, 10 further educational institutions can be included (by offering flat-rate cooperation grants of $€ 1,000$ ) and the regional networks can be expanded. In the period from 2011 to 2019, 141 Talents Regional projects were realised with a focus on topics in the fields of energy and environment, life sciences, IT and mobility. The children and young people participating in the projects explored exciting topics, experimented, researched and learned about activities in research, technology and innovation.

The main effect of the Talente Regional projects is to initiate the children's and young people's spirit of research and enthusiasm for science and technology. Knowledge and interest in these areas have increased, and the target group could gain a realistic insight into the work of researchers and technicians. The teachers involved benefit above all from learning new pedagogical concepts and innovative teaching methods. At the level of schools, the importance of science and technology is strengthened and the technical infrastructure is improved. Another effect of the Talents Regional projects is that sustainable regional cooperation between education, science and the business sector is created, which was the case for $72 \%$ of the surveyed project participants after the end of the projects.

However, the required cooperation activities also entail a high organisational effort in the project implementation. The programme line's claim to innovation as well as the additional focus on gen- 
der balance and the integration of children and young people regardless of their social or geographical origin are also perceived as challenging. This could be remedied by making the instrument more flexible, e.g. by setting a clearer focus on certain age groups (e.g. only preschool and primary schoolchildren, only lower secondary school) as well as by softening the innovation requirement. Thus, successful projects could be permitted for renewed funding in an adapted form in another setting. This could also be beneficial to the sustainability of the projects, as successful and already tested concepts could be implemented again.

The instrument can be assessed a success and the demand is extremely high. Thus, an annual call for proposals with a corresponding increase in funding and the resulting larger number of funded projects could reach more educational institutions and children / young people. This would allow more regions to benefit from the positive effects and ensure greater continuity in the interventions - both are supporting factors with regard to structural changes.

\section{FEMtech Internships for Female Students}

FEMtech Internships for Female Students specifically support young female scientists in gaining ground in applied research in science and technology. During the internships, which last up to 6 months, the students learn about career entry and advancement paths and gain an insight into applied research. In the period from 2011 to 2019, around 3,600 funded internships for female students were approved. The majority of the funded internships are offered in non-university research institutions (58 \%) and $42 \%$ take place in companies. Companies predominantly offer a single internship for female students, while research institutions often accept several female students as interns at the same time.

Both, funding recipients and students can benefit from the funded internships. Female students have the opportunity to gain insight in the professional field and gain professional experience, and at the same time, the companies / research institutions benefit by getting to know potential female employees in advance. After the internship, about three quarters of the female students showed an increased interest in working in research, science and technology. The internships also make it easier for female students to start their careers. In 2018, around a quarter of the female students were able to continue working for the respective organisation after the internship period has ended. The funded internships thus facilitate the entry into the working world for female students and help them to gain ground in a profession in the field of research, science and technology in the long term.

The only drawback in connection with the instrument is the rapid exhaustion of funding and the usually early closure of the call for proposals. In most cases, companies are at a disadvantage here because research institutions usually submit several applications for funding for female interns right at the beginning of the call period. In order to support internships in companies, it is therefore proposed to cap the number of funded internships per organisation, so that more organisations can benefit from the funding and the instrument is spread more widely. In addition, a call for proposals in two independent phases, as was done in 2019, could lead to a further spread among the funding recipients, and would allow the internships to be even better adapted to the needs of the funding recipients. 


\section{FEMtech Career and FEMtech Career Check for SMEs}

To promote equal opportunities, the FEMtech Career programme line supports organisations in employing more women in the fields of science and technology and in creating good and fair framework conditions for all employees. In 2015 and 2016, the FEMtech Career Check for SMEs was available to enable this group of companies to conduct a gender analysis and prepare them for a possible subsequent FEMtech Career project. In the period from 2011 to 2019, 34 FEMtech Career projects and 17 FEMtech Career Check projects for SMEs were implemented. The FEMtech Career Check for SMEs provided companies with a good introduction to the topic, as the majority of these companies (12 out of 17 or $71 \%$ ) subsequently submitted a FEMtech Career Project successfully. Within the framework of the FEMtech Career projects (duration 6 to 24 months), participating organisations deal with various modules in the fields of gender competence, equal opportunities and the advancement of women. Increasing the attractiveness of the funded organisation as an employer is also an important objective of the projects. Organisations that are already familiar with gender issues and the FFG funding tend to participate more likely in the projects.

The effects of the FEMtech Career projects can be seen above all in an increase in gender competence and in the increased acceptance and awareness of the topic in the participating organisations. The nomination of gender equality officers, which took place in 11 of the 17 organisations surveyed, also ensures a sustainable implementation of the gender issue. Further observable effects are an optimised external image and the increase of the attractiveness as an employer, the improvement of working conditions and the work-life balance in the organisations as well as the successful qualification and career planning activities of women. Increases in the proportion of women and female researchers, on the other hand, are only rarely evident. The instrument can primarily be seen as a measure to raise awareness.

However, there is little demand for the instrument and the applicants usually already have a high awareness for the topic. The requirements are often too complex for newcomers. To create real structural changes in the framework conditions for equal opportunities in science and technology, FEMtech Career projects would have to be carried out in considerably more companies and research institutions in order to generate a critical mass of "gendered" organisations and subsequently spillover effects. The biggest challenge is therefore to attract more companies and research institutions to the instrument. This would require broad mobilisation measures that go far beyond the "Talents clientele", and would include the typical FFG funding recipients. This would also need a corresponding substantial increase in financial resources for PR measures and target group mobilisation for an instrument that only unfolds its effect in the medium to long term. Even if these funds are not made available, good practice examples and success stories of funded projects should in any case be made more widely accessible to all FFG clients. In addition, possible synergy effects with the BMK's FEMtech initiative for mobilising target groups should be examined.

In addition to general mobilisation measures, experts suggest changing the name of the programme line. The name "FEMtech Career" suggests that the programme only helps women to make a career. However, the aim of the instrument is to change the corporate culture so that the 
organisation prepares itself for the future and achieves a competitive advantage in the competition for skilled workers by creating fair and good working conditions for all. The name should reflect this aim. In addition, the possibility of developing a Gender Equality Plan within the project should be fostered, which will increasingly be required in (international) funding programmes, or be a good reference. Finally, the introduction of a new tool for newcomers should be discussed in order to introduce gender-inexperienced organisations to the topic. This will enable them to acquire the gender competence needed for a successful FEMtech Career application.

\section{FEMtech Research Projects}

FEMtech Research Projects is a unique programme line, both within Austria and internationally, and enjoys good reputation within the (international) gender research community. The USP of FEMtech Research Projects compared to other project funding programmes is the strong emphasis on the gender dimension; only projects with gender-relevant content are funded. Therefore, the programme line contributes to the goal of Talents to initiate RTI projects with gender-relevant content. The programme line is very popular among funding recipients, and demand is correspondingly high: from 2011 to 2018 , only $23 \%$ (52 projects) of the 231 projects submitted were approved for funding. The Austrian Research Promotion Agency (FFG) has reacted to the recommendations of the review from 2016, and the call guidelines were revised from the fifth call onwards.

Since the aim of the programme line is also to increase the quality of products (to be developed) by integrating the gender dimension, the project results and project management processes can be of interest to the field of RTI as a whole. As stated in the recommendations of previous analyses, we emphasise the need to foster dissemination activities of the programme line. In order to achieve a more effective marketing of economically successful projects and a reflection of the research and development process, as well as structural changes in organisations and the research process, dissemination must go beyond the Talents programme and the programme line respectively and also make use of other events and thematic platforms. We suggest increasing networking with other programmes in the FFG in order to stimulate suitable dissemination opportunities. Another possibility would be to integrate the gender dimension in the context of other research funding programmes to a greater extent, for example by giving greater weight to gender criteria in the context of evaluation procedures or reserving part of the funding for projects with an explicit gender focus.

\section{Career Grants}

The Career Grants programme line is unique within the FFG funding portfolio as the target group for funding are individual researchers. The aim of the funding is to attract researchers from abroad to the field of RTI in Austria by means of financial support. Funding is provided for the costs of job interviews in Austria as well as relocation and integration costs for researchers and their partners. Out of the 2,241 submissions made in the period under review, 1,903 were funded. Overall, up to the 2019 call, $57 \%$ of funded submissions were Relocation Grants, 38\% Interview Grants, and 5\% Dual Career Grants. Male researchers are more likely to make use of Career Grants than female researchers. The majority of researchers were in Europe at the time of their application. 
Overall, most of the funded individual researchers were very satisfied with the funding, and the increase in the number of applications over the years indicates a corresponding demand. However, with regard to the achievement of objectives, the funding has a very low impact. Taking up a job in Austria is hardly influenced at all by the Relocation Grants; for more than $90 \%$ of the interviewees, this is not the case. The Interview Grants are most likely to have an effect. About a quarter $(23 \%)$ of the respondents stated that they would not have or probably would not have come to Austria for the job interview without the Interview Grant. Of these, however, around 36\% would have held the interview online alternatively.

Therefore, the instrument must be called into question in principle, especially since attracting researchers from abroad depends primarily on the attractiveness of the research location and the general framework conditions, and a financial contribution for interview or relocation costs has practically no influence on the decision. To stop the funding is also recommended against the background of the oversubscription of other successful programme lines within Talents. Should the instrument nevertheless be sustained, a restriction of the target group of the Relocation Grant to researchers in geographically more distant regions, possibly coupled with socio-economic indicators (such as the median income of researchers in the country of origin), should be considered. In this case, the maximum funding amount should also be adjusted, i.e. increased or differentiated accordingly. Concerning the Dual Career Grant, the question arises about the objective of the funding and whether it can be achieved, as the grant plays no role in the decision to move with the partner.

\section{The Austrian Job Exchange for Research, Development and Innovation}

The Job Exchange is a service that should facilitate the search for (highly) qualified specialists by companies or research institutions and the search for jobs in the field of research, technology and innovation by specialists. In the period from 2013 to 2018, the website had an average of around 5,300 visitors per quarter, so the target value was not achieved. In the fourth quarter of 2019 , the target of 6,000 visitors was reached. The same was true in the first quarter of 2020 , followed by a decline in the second and third quarter of 2020 (Corona crisis). The increase in posted job advertisements on the Job Exchange website during the observation period indicates an increased use by organisations. Based on the available information, it could not be conclusively clarified whether the Job Exchange provides an additional benefit for both job seekers and job providers that is not already covered by other service offers. Therefore, it is recommended to conduct a user survey to find out whether the Job Exchange has a benefit for its users and contributes to the goals of the Talents programme. For this purpose, the registered companies whose websites are regularly screened for job advertisements could be surveyed, as well as the visitors of the job exchange after using the site, for example by means of an onsite survey.

\section{Support measures}

Various support measures are implemented as part of the Talents programme: award events in the programme line Internships for Students, networking events as part of Talents Regional, information events for FEMtech Research Projects. These measures primarily support the mobilisation of the target groups and the visibility and dissemination of activities. Additionally, there are the FEMtech network meetings. 
In the programme line Internships for Students, each year the 20 students with the best internship reports receive a prize at the award ceremony. In case the organisation and the student agree with the publication, the internship reports could also be published online, and could be used for information dissemination and customer acquisition as well. In order to strengthen the networking events of Talents Regional, we recommend to focus on the presentation of good practice examples and on the exchange with experienced funding recipients, as well as to offer an online format to enable more organisations to participate in the event. The two information events for FEMtech research projects 2017 and 2018 have been very well attended, which speaks for the high information needs on the part of potential funding recipients. Due to the Corona pandemic, the third information event for the seventh call was held online. We suggest to offer such an online format (additionally) in the future in order to give more interested organisations the opportunity to participate and to be able to present more FEMtech research projects.

The FEMtech network meetings are organised to inform about news from the Talents programme and to highlight current FEMtech topics. There is the possibility for informal exchanges and funding advice from the FFG. People involved in equal opportunities in research, science and technology mainly attend the meetings. Thus, a broad dissemination of the topic in the research community cannot be expected. In order to put the topic of equal opportunities in research on the general agenda and for a broader mobilisation of applicants, we suggest broadening the circle of interested parties by opening up the format (with view to e.g. invitation policy, dissemination of event reports etc.). The meetings could then better serve to disseminate information about the funding formats, especially about FEMtech Career.

\section{Summary and conclusion}

The design of the programme meets its objective to support the entire career path and defines this in terms of a very broad target group that includes potential researchers and thus all children and young people. It addresses neuralgic phases in the career (e.g. educational decisions, career choice, career entry) and tries to influence them through interventions. In addition, the programme aims at structural changes to prepare the ground for better utilisation of human potential in the application-oriented, scientific and technical RTI sector. As a programme for human potential, the Talents programme is not only an exception in the FFG portfolio, but also improves the impact of other research funding programmes indirectly and in the long run by supporting researchers, who then can make better use of their talents in various projects.

The broad scope of the programme is an important characteristic, but it bears the risks of overloading with objectives and a lack of focus against the background of limited resources. In particular, the field of intervention "Professional Talents" requires a critical analysis from the evaluation team's point of view. The instruments seem only partially suitable for achieving the goals and the impact is very low or cannot be comprehensively assessed. We therefore recommend questioning this field of intervention with regard to its objectives and the instruments used fundamentally. Based on the discussion, the field of intervention should be either redesigned or abandoned in favour of the other fields of intervention. With the focus on the promotion of young researchers and equal opportunities, the resources could be used in a more concentrated way, but the programme would have to make concessions with regard to its comprehensive approach. 
Mobilising the target groups is essential for a "non-classical" research funding programme. On the one hand, FFG newcomers should be recruited to the programme to a large extent; on the other hand, it is important to attract typical FFG clientele for instruments that tend to have effects mainly in the medium- to long-term run and are often not directly visible. The dissemination of information is therefore of great importance. Thus, the expansion of mobilisation measures is crucial to support a broad impact of the programme.

Broad impact is also hindered by the fact that the entire programme under its name "Talents" is still not known as such, in contrast to the individual programme lines or fields of intervention (e.g. FEMtech), which are a familiar term for many funding recipients and for most experts. It could be considered to strengthen the "branding", whereby the name of the programme lines should include the programme name. When naming the programme lines, it should be taken into account that the name adequately describes the instrument (see FEMtech Career). If the programme gains brand recognition, this could help support its mission of developing human potential for applied research. The rebranding of the entire programme, through which the affiliation of all programme lines to the programme becomes clearly visible, should be accompanied by a public relaunch.

In conclusion, it must be stated that a programme like Talents, which addresses the structures and framework conditions and wants to change nothing less than established attitudes and roles, can only make a small contribution with its limited resources. The importance of promoting human potential must also find real acceptance in the research community and be more than just lip service. The figures are still sobering, in particular with regard to the representation of women in the research sector - and especially in the industrial sector. Therefore, a revaluation and mainstreaming of the topic within the FFG and the BMK is needed. The topics of human resources and equal opportunities must be understood as cross-cutting issues and be taken into account accordingly in other programmes, and their information channels should be used for the Talents programme as well. 


\section{3 | Einleitung}

\section{1 | Hintergrund und Ziele der Evaluierung}

Die Entwicklung von Humanpotentialen in Forschung, Technologie und Innovation (FTI) ist eine der größten Herausforderungen sowohl in Österreich als auch im europäischen Raum, um die Forschungs- und Innovationsaktivitäten zu gewährleisten. Dabei gilt es - vor dem Hintergrund des steigenden Bedarfs an Fachkräften insbesondere im naturwissenschaftlich-technischen Bereich, die verfügbaren Humanpotentiale quantitativ zu erweitern und ihre Qualität zu steigern.

Unter der Annahme, dass die Talente grundsätzlich vorhanden sind, wurden die Aktivitäten zur Förderung der Humanpotentiale im Bundesministerium für Klimaschutz, Umwelt, Energie, Mobilität, Innovation und Technologie (vormals BMVIT) gebündelt und verstärkt und 2011 unter dem Förderschwerpunkt Talente subsumiert. Im Rahmen dieses Förderschwerpunkts werden die Menschen in der angewandten Forschung über den gesamten Karriereverlauf, vom Kind zur/zum etablierten Forscherln unterstützt. Übergeordnetes Ziel ist die erhöhte Ausschöpfung des Humanpotentials im anwendungsorientierten, naturwissenschaftlich-technischen FTI-Bereich, die drei Zielsetzungen sind

- junge Menschen für Forschung und Entwicklung zu begeistern,

- Forscherinnen und Forscher mit der Wirtschaft vernetzen und

- gleiche Chancen für alle garantieren.

Zielgruppe sind die (potentiellen) Forscherlnnen in ihrem gesamten Karriereverlauf (unmittelbare Zielgruppe), die über einreichberechtigte FörderungswerberInnen angesprochen werden. Die einreichberechtigten FörderungswerberInnen - Unternehmen, Universitäten und Fachhochschulen, außeruniversitäre Forschungseinrichtungen, Intermediäre und Einrichtungen des Technologietransfers, jeweils mit Standort in Österreich - können als erweiterte Zielgruppe ${ }^{1}$ verstanden werden.

Der Förderschwerpunkt Talente hat drei Interventionsfelder, in denen jeweils mehrere Instrumente zur Anwendung kommen:

Talente entdecken: Nachwuchs

- Praktika für Schülerinnen und Schüler: Hochwertige Sommerpraktika im Bereich Naturwissenschaft und Technik sollen als Bildungs- und Orientierungsangebot sowie als Impulsgeber für eine entsprechende Studien- und Berufswahl dienen.

- Talente regional: In einer Region bieten (vor-)schulische Bildungseinrichtungen und Organisationen aus Forschung und Wirtschaft gemeinsame Aktivitäten für Kinder und Jugendliche (von Kindergartenalter bis zur Matura) zur Auseinandersetzung mit FTI-Themen (Forschung, Technologie, Innovation) an.

${ }^{1}$ Ausnahme sind die Karriere-Grants - hier sind Einzelforscherlnnen auch Fördernehmerlnnen. 
- FEMtech Praktika für Studentinnen: In geförderten hochwertigen Praktika und aktiver Mitarbeit an Forschungsprojekten in Unternehmen und außeruniversitären Forschungseinrichtungen im naturwissenschaftlich-technischen Bereich sollen Studentinnen praxisbezogenes Know-how vermittelt und an die angewandte Forschung herangeführt werden.

- FEMtech Karriere: Strukturelle und nachhaltige Maßnahmen zur Chancengleichheit von Frauen und Männern in Unternehmen und außeruniversitären Forschungseinrichtungen im naturwissenschaftlich-technischen Bereich werden gefördert.

- FEMtech Karriere-Check für KMU (2015-2018): Kleine und mittlere Unternehmen (KMU) wurden bei der Erstellung einer von externen Expertinnen und Experten durchgeführten Genderanalyse sowie daraus abgeleiteter Empfehlungen und Maßnahmen zur Verbesserung der Chancengleichheit im Unternehmen gefördert.

- FEMtech Forschungsprojekte: Forschungsprojekte mit Genderrelevanz, deren Forschungsgegenstand die unterschiedlichen Lebensrealitäten und Bedürfnisse von Frauen und Männer berücksichtigt, werden gefördert und damit Innovationen und die Erschließung neuer Marktpotentiale unterstützt.

\section{Talente finden: Forscherinnen und Forscher}

- Karriere-Grants: Durch die gezielte Übernahme von Kosten, die bei einer Standortveränderung entstehen, werden EinzelforscherInnen, die im Ausland leben und am Forschungsstandort Österreich arbeiten wollen, unterstützt.

- Jobbörse für Forschung, Entwicklung und Innovation: in der frei zugänglichen und kostenlos nutzbaren Jobbörse sind freie Stellen in der österreichischen Forschung und Entwicklung und mit Innovationsbezug veröffentlicht.

Zudem finden im Rahmen von Talente diverse Begleitmaßnahmen, wie FEMtech Netzwerktreffen, Prämierungsveranstaltungen etc. statt. Der Förderschwerpunkt wird von der Österreichischen Forschungsförderungsgesellschaft mbH (FFG) abgewickelt.

Im Jahr 2013/2014 wurde eine Zwischenevaluierung durchgeführt und ein neues Programmdokument erstellt, in der für 2020 eine Endevaluierung avisiert ist. Diese umfasst eine Analyse der Konzeption, Umsetzung, Zielerreichung und Wirkungen des Programms sowie Schlussfolgerungen und Empfehlungen für die Weiterentwicklung des Förderschwerpunkts Talente.

Der vorliegende Evaluierungsbericht ist wie folgt aufgebaut: Zunächst wird das Design des Programms analysiert (Kap. 3) sowie die Organisation und Abwicklung (Kap. 4). Die einzelnen Programmlinien und ihre Wirkungen werden in Kap. 5 beschrieben. Kap. 6 bietet eine Gesamtbetrachtung des Programms. Basierend darauf werden Schlussfolgerungen und Empfehlungen zur Weiterentwicklung des Förderschwerpunkts Talente formuliert (Kap. 7). Wörtliche Zitate stammen aus den Feedback-Fragebögen der FördernehmerInnen sowie der SchülerInnen und Studentinnen bei den Praktika für Schülerinnen und Schüler und den FEMtech Praktika für Studentinnen, aus Interviews und Fokusgruppen, die im Rahmen der Fallstudien über FEMtech Karriere 
Projekte erstellt wurden. Auch Antworten auf offene Fragen aus der Online-Befragung der FEMtech Karriere Projekte, werden wörtlich zitiert, um die Projektabwicklung anschaulich darzustellen.

\subsection{Methodik}

Die Evaluierung des Förderschwerpunkts Talente stützt sich auf eine Kombination aus quantitativen und qualitativen Methoden. Dabei wurde darauf Wert gelegt, auf verfügbare Datenquellen zurückzugreifen und diese optimal zu nützen und Primärerhebungen ergänzend zur Generierung zusätzlicher Informationen einzusetzen. Die folgenden Methoden fanden im Rahmen der Evaluierung Anwendung:

\section{Dokumentenanalyse}

Im ersten Schritt wurden vorhandene Dokumente gescreent und analysiert sowie die für die Evaluierung relevanten Informationen herausdestilliert. Untersucht wurden folgende Dokumente:

- Programmdokument

- Jahresberichte des Programms

- Schlussauswertungen einzelner Programmlinien (Praktika für Schülerinnen und Schüler, FEMtech Praktika für Studentinnen)

- Endberichte bzw. Zwischenberichte der Projekte (Talente regional, FEMtech Karriere, FEMtech Forschungsprojekte)

- Reviews der Programmlinien Talente regional (ibw, 2018) und FEMtech Forschungsprojekte (IHS, 2016)

\section{Datenanalyse}

Es erfolgte eine Analyse der Projektdaten aus der FFG-Datenbank. Darüber hinaus beinhaltete die Datenanalyse auch die Untersuchung der Auswertungen der Feedbackfragebögen der FördernehmerInnen und PraktikantInnen von Praktika für Schülerinnen und Schüler sowie FEMtech Praktika für Studentinnen.

\section{Interviews mit Expertinnen und Experten}

Mit Expertinnen und Experten wurden qualitative Interviews durchgeführt. Die Interviews erfolgten persönlich oder telefonisch auf Basis eines semi-strukturierten Interviewleitfadens. Folgende Personenkreise wurden interviewt:

- Verantwortliche Personen im BMK (2 Personen)

- Verantwortliche Personen in der FFG (7 Personen)

- FachexpertInnen zum Thema Humanressourcen in Forschung und Technik, insbesondere im Bereich Gender und Nachwuchsförderung sowie Jurymitglieder (10 Personen) 


\section{Quantitative Online-Befragungen von FördernehmerInnen}

Fördernehmerlnnen folgender Programmlinien wurden online befragt:

- FEMtech Karriere / FEMtech Karriere-Check für KMU: Eine Einladung zur Befragung wurde per E-Mail Ende August 2020 an 35 FördernehmerInnen versandt, davon konnte eine Organisation nicht mehr erreicht werden. Am Mitte September 2020 erfolgte ein Erinnerungsschreiben per E-Mail an diejenigen, die den Fragebogen bis dahin noch nicht ausgefüllt hatten. Um die Rücklaufquote zu erhöhen, wurden die Organisationen auch per telefonischer Nachfrage gebeten, die Umfrage noch auszufüllen. Insgesamt konnten so 20 ausgefüllte Fragebögen verwertet werden, was einer Rücklaufquote von 59 \% entspricht.

- Karriere-Grants: Eine englischsprachige Einladung zur Befragung wurde Mitte August 2020 an 1.647 Fördernehmerlnnen auf Basis der von FFG zu Verfügung gestellten Kontaktdaten (Ausschreibungen von 2011 bis 2019) versandt. Ende August 2020 erfolgte eine E-Mail-Erinnerung an diejenigen Fördernehmerlnnen, die den Fragebogen bis dahin noch nicht ausgefüllt hatten. Die Befragung wurde am 09.09.2020 geschlossen. Insgesamt konnten 1.433 FördernehmerInnen erreicht werden (214 E-Mai-Adressen erwiesen sich als ungültig), 740 Fragebögen wurden vollständig ausgefüllt, was einer Rücklaufquote von rd. $52 \%$ entspricht.

\section{Fallstudien}

Zur Analyse der Programmlinien FEMtech Karriere wurden sechs Fallstudien (siehe Anhang) von geförderten Projekten durchgeführt, wobei eine Organisation drei Karriere-Projekte (eines laufend) durchgeführt hat, zwei Organisationen zwei Karriere-Projekte und drei Organisationen vor dem Karriere-Projekt einen Karriere-Check für KMU. Für die Fallstudien wurden die Projektberichte analysiert und Interviews mit den ProjektleiterInnen, der Geschäftsführung sowie Interviews/Fokusgruppen mit MitarbeiterInnen durchgeführt, wobei InterviewpartnerInnen teilweise mehrere Rollen eingenommen haben (z. B. Geschäftsführung und Projektleitung, Projektleitung und Beschäftigte/r). Insgesamt wurden 18 Personen telefonisch oder online befragt.

Die Durchführung der Fallstudien hat sich als sehr herausfordernd erwiesen, da aufgrund von Personalfluktuationen einige ProjektleiterInnen, und besonders bei kleinen Organisationen teilweise die MitarbeiterInnen, die das Karriere-Projekt „miterlebt“ haben, gar nicht mehr in der Organisation tätig oder gerade in Karenz waren.

\section{Workshops}

Zur Diskussion der vorläufigen Ergebnisse und zur Erarbeitung von Schlussfolgerungen und Empfehlungen zur Weiterentwicklung des Programms wurden zwei Online-Workshops durchgeführt. Um die Diskussion zu fokussieren und konkrete Handlungsoptionen zu erarbeiten, hatten die Workshops verschiedene Schwerpunkte. Einer befasste sich mit der Zielgruppe Kinder und Jugendliche, der andere mit dem Thema Chancengleichheit. An den Workshops teilgenommen haben Verantwortliche aus dem BMK und der FFG (zwei Personen je Workshop) sowie externe ExpertInnen (jeweils drei Personen). 


\section{4 | Design des Förderschwerpunkts}

Auf strategischer Ebene soll das Programm Talente zur Entwicklung von Humanpotentialen in Forschung, Technologie und Innovation beitragen. Den Rahmen bildet hierfür die FTI-Strategie des Bundes (2011), die sich zum Ziel setzt, Österreich als einen Innovation Leader innerhalb Europas zu positionieren. Rechtlicher Rahmen hierfür sind die beiden FTI-Richtlinien „ThemenFTI-Richtlinie“ und „Humanressourcen-FTI-Richtlinie“.

Der Förderschwerpunkt Talente ist ein umfassendes Humanressourcenprogramm, dessen strategisches Ziel die Unterstützung von (potentiellen) ForscherInnen über den gesamten Karriereverlauf ist. Damit soll es zu einer bestmöglichen Nutzung und Ausschöpfung des Humanpotenzials im anwendungsorientierten, naturwissenschaftlich-technischen FTI-Bereich kommen. Wie dieses strategische Ziel in operative Ziele und Instrumente mit entsprechendem Output übertragen wurde, und welche Wirkungen erzielt werden sollen, ist in der Interventionslogik (siehe unten) dargestellt.

Dabei wird ersichtlich, dass das Programm drei Hauptzielgruppen hat:

- Kinder und Jugendliche, die als potentielle ForscherInnen an das Feld herangeführt und für diesen Karriereweg begeistert werden sollen.

- NachwuchsforscherInnen, insbesondere Frauen, die über ihre Studienwahl bzw. Berufswahl schon einen ersten Schritt ins Feld gemacht haben und nun langfristig für die naturwissenschaftlich-technische Forschung gewonnen werden sollen.

- ForscherInnen aus dem Ausland, die für die anwendungsorientierte Forschung in Österreich gewonnen und an diese gebunden werden sollen.

Damit wurde dem Anspruch, den gesamten Karriereverlauf der Forscherlnnen zu unterstützen, nicht nur gerecht, der Ansatz ist auch sehr breit gewählt, da neben etablierten Forscherlnnen und NachwuchsforscherInnen auch Kinder und Jugendliche als potentielle ForscherInnen adressiert werden, die noch vor der Bildungs- und Berufswahl stehen. Neben diesen Individuen als Zielgruppe richtet sich das Programm auch an Organisationen (Unternehmen, Forschungseinrichtungen, Bildungseinrichtungen), die auch die Fördernehmerlnnen sind (Ausnahme: KarriereGrants). Entsprechend breit ist das Instrumentenportfolio. Es reicht von der Förderung von Praktika über die Förderung von Projekten zur Bewusstseinsbildung und Organisationsentwicklung sowie von Einzelforscherlnnen bis zur „klassischen“ Förderung von Forschungsprojekten.

Das Programm fokussiert auf die neuralgischen Phasen in der Forschungskarriere bzw. setzt auch schon vor der eigentlichen Karriere bewusstseinsbildende Maßnahmen. Neben dieser Förderung und Stärkung des Individuums, setzt das Programm mit einzelnen Instrumenten gleichzeitig an den Strukturen an und zielt auf eine Veränderung der Organisationskultur in der angewandten Forschung, um das Feld attraktiver für potentielle ForscherInnen zu machen. Dies macht das Programm zu einem sehr breiten, umfassenden und vielseitigen Programm. Dieser holistische Ansatz manifestiert sich auch in der Verankerung von Gender- und Diversity-Kriterien im Interventionsfeld Nachwuchs sowie in der Integration der FEMtech Forschungsprojekte im Programm. Dieses Instrument stellt zwar eine klassische Forschungsprojektförderung dar, da die 
Projekte aber Genderinhalte im Fokus haben müssen, tragen sie einem sehr umfassenden Bild von Gender Mainstreaming in der Forschung Rechnung. ${ }^{2}$ Zudem muss im Projektteam Genderexpertise nachgewiesen werden.

Das Programm Talente stellt im FFG-Portfolio als Humanpotentialprogramm somit eine Ausnahme dar. Der Mensch und nicht der Forschungsinhalt (mit Ausnahme von FEMtech Forschungsprojekte) steht im Mittelpunkt. Somit sind die unmittelbaren Wirkungen auch in weiten Teilen auf individueller Ebene zu verorten, wie ein gesteigertes Interesse an FTI und die Entscheidung zu einer entsprechenden Ausbildung oder die praktisch gemachten Erfahrungen im Feld. Für ForscherInnen liegt der Output im vermittelten Job in Österreich. Aber auch auf Ebene der Organisationen sollen Wirkungen erzielt werden, indem ein gendersensibles und förderliches Karriereumfeld geschaffen wird. Dieses kommt dann wieder den Individuen, die im FTI-Bereich arbeiten zugute.

Durch eine Steigerung der Anzahl der MINT-AbsolventInnen und mehr Diversität unter diesen soll das Programm in weiterer Folge dazu beitragen, dass das Potential an Menschen, die in der naturwissenschaftlich-technischen Forschung arbeiten können, steigt und sich mehr Menschen für eine Karriere in der anwendungsorientierten Forschung entscheiden. Diese sollen dann auf ein förderliches Umfeld mit guten Entwicklungsmöglichkeiten treffen - auf Unternehmen und Forschungseinrichtungen, in denen Chancengleichheit gelebt wird und die ihre Rekrutierungsmöglichkeiten verbreitern.

Die eingesetzten Instrumente können hier einen Beitrag leisten, aber es ist zu bedenken, dass Bildungs- und Berufsentscheidungen von vielen Einflussfaktoren abhängig sind und z. B. die Teilnahme an einem Praktikum oder einem Workshop nur einen wichtigen Impuls erzeugen kann. Je früher in der Bildungskarriere die Interventionen ansetzen, desto unsicher sind die konkreten Auswirkungen zudem. Auch können in einem FEMtech Karriere Projekt wichtige strukturelle Weichen in einem Unternehmen gestellt werden, aber eine Änderung der Unternehmenskultur erfolgt durch die gelebte Praxis, die wiederum von den äußeren Rahmenbedingungen beeinflusst wird.

2 Gemäß dem Prozess der "gendered innovations“ und den Instrumenten "fixing the numbers“, ,fixing the institutions und „fixing the knowledge“ (Schiebinger/Schraudner, 2011, http://genderedinnovations.stanford.edu/ISR 07 Schiebinger.pdf) 


\section{Programm Talente - Strategische Ziele}

- Unterstützung von Menschen in der angewandten Forschung über den gesamten Karriereverlauf

- Erhöhte Ausschöpfung und bestmōgliche Nutzung des Humanpotentials im anwendungsorientierten, naturvissenschaftlich-technischen FTI- Bereich

- Förderung junger Nachwuchsforscherinnen und deren Vernetzung mit der Wirtschaft, Chancengleichheit

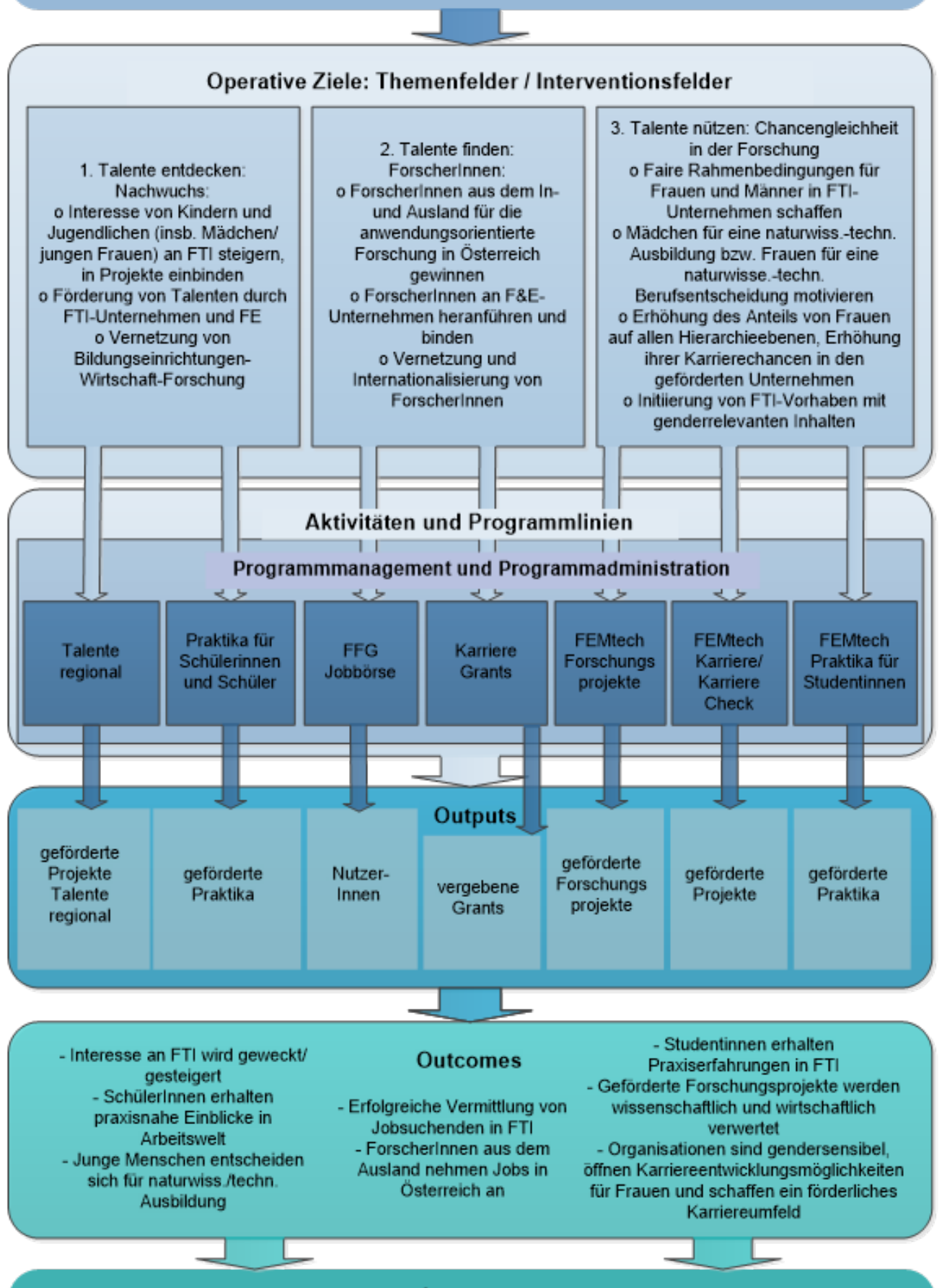

\section{Impacts}

- Anzahl an MINT-Absolventinnen steigt

- Höherer Anteil Frauen an MINT-Absolventinnen

- Mehr Personen entscheiden sich für eine Karriere in der Forschung

- Bessere Entwicklungsmöglichkeiten junger Menschen und effektivere Verwertung von Talenten in der Forschung - Bessere Rekrutierungsmōglichkeiten von Personen im FTI-Bereich

- Entwicklung innovativer Produkte, Services und Prozesse mit Gender-Bezug

Innovationskraft am Standort Österreich wird erhöt

- Bildung neuer sozialer Praktiken und Strukturen auf Meso- und Makroebene, welche Chancengleichheit fordern 
Grundsätzlich gibt es in Österreich kein Programm in dieser Breite, dass die gesamte Karriere der ForscherInnen im Fokus hat und die Zielgruppe (potentieller) ForscherInnen so umfassend definiert. Damit grenzt sich Talente von vergleichbaren Programmen mit überschneidenden Zielgruppen ab. Es gibt Programme und Initiativen, die die Heranführung von Kindern und Jugendlichen an die Forschung zum Ziel haben, aber in erster Linie in den Schulen ansetzen und Projekte in den Schulen fördern (z. B. Sparkling Science, Vienna Open Lab von Open Science). Mit den Praktika für Schülerinnen und Schüler und Talente regional erfolgt eine Einbindung von Unternehmen sowie Forschungseinrichtungen und die Kinder und Jugendlichen können praktische Erfahrung im Unternehmenskontext sammeln.

Die Zielgruppe Frauen in Forschung und Technologie teilt sich Talente z. B. mit w-fFORTE, das mehr Frauen in gestaltenden Rollen in der standortrelevanten Forschung und Innovation sichtbar machen will. In dessen Rahmen sollte mit dem Impulsprogramm Laura Bassi Centres of Expertise eine neue Forschungskultur in der kooperativen Forschung implementiert und die strukturelle Verankerung von Chancengleichheit in der Community unterstützt werden. FEMtech Karriere fördert die nachhaltige Implementierung von Chancengleichheit in der angewandten naturwissenschaftlich-technischen Forschung durch die Förderung umfassender Maßnahmen in einzelnen Unternehmen und Forschungseinrichtungen auf Basis einer umfassenden Genderanalyse (diese wurde auch im Rahmen von FEMtech Karriere-Check für KMU gefördert). Auch fördert FEMtech Praktika für Studentinnen Unternehmen und Forschungseinrichtungen, die Studentinnen einen Praktikumsplatz anbieten. Damit ist das Instrument nicht nur für Frauen reserviert, sondern auch wesentlich niederschwelliger als z. B. die Kostenunterstützung Junge ForscherInnen, das den KMU einen Kostenzuschuss für Diplomandlnnen, DissertantInnen, Bachelors, Masters, Junior Researcherlnnen und Post Docs innerhalb eines Forschungs- und Entwicklungsprojekts zugesteht.

Ein anderes Programm zur Förderung von Nachwuchsforscherlnnen ist Forschungspartnerschaften - industrienahe Dissertationen, das Dissertationsprojekte in Unternehmen und außeruniversitären Forschungseinrichtungen fördert und die angewandte Forschung für Berufseinsteigerlnnen öffnen soll sowie die Höherqualifizierung im Unternehmen forciert. Der systematische Aufbau und die Höherqualifizierung des bestehenden Forschungspersonals ist Förderungsgegenstand des Programms Forschungskompetenzen für die Wirtschaft. Talente dagegen konzentriert sich darauf, potentielle ForscherInnen für die angewandte Forschung zu begeistern und zu gewinnen bzw. mit der Wirtschaft zu vernetzen sowie durch die Förderung geeigneter Rahmenbedingungen die Karriereentwicklung voranzutreiben.

Karriere-Grants ist eine Individualförderung, die eine Kostenunterstützung für ForscherInnen für ein Vorstellungsgespräch bzw. den Umzug nach Österreich bei Dienstantritt bietet. Damit grenzt sich das Instrument von vielen Individualförderungen ab, die meistens Reisekosten, die im Rahmen eines Forschungsprojekts bzw. der Dissertation etc. entstehen, abdecken (z. B. Stipendienprogramme des ÖAD, ÖAW, FWF). Oftmals gibt es hier auch eine Fokussierung auf Fachdisziplinen und engere Zielgruppen. 
Als Besonderheiten des Programms können daher festgehalten werden: die Breite des Programms mit einem entsprechend ausdifferenzierten Instrumentenportfolio und einer umfassenden Definition der Zielgruppe, die Betrachtung verschiedenster Phasen der Karriere von (potentiellen) ForscherInnen, Individuen und Organisationen sensibilisierende Instrumente, strukturverändernde Maßnahmen. Diese Merkmale stellen die Stärken des Programms dar, bedeuten aber auch besondere Herausforderungen.

Die Breite des Programms und die verschiedenen Zielgruppen bergen die Gefahr der Überfrachtung in Hinblick auf die Zielsetzungen. Zudem müssen die Ressourcen (Budget und Abwicklung in der FFG) auf das breite Instrumentenportfolio aufgeteilt werden, mit der Gefahr, dass keines der Instrumente die entsprechenden Ressourcen und die Aufmerksamkeit hat, um breitenwirksam zu werden und es bei eher punktuellen Interventionen bleibt. Instrumente, die sehr früh in bzw. vor der Karriere ansetzen, wirken langfristig und im Zusammenspiel mit einer Vielzahl anderer Einflussfaktoren. Und schließlich ist ein solches Programm, das auf Strukturveränderungen abzielt, besonders von gesamtgesellschaftlichen Rahmenbedingungen im Allgemeinen und den Rahmenbedingungen im FTI-Bereich im Besonderen abhängig. So kann ein Programm wie Talente auf "fruchtbaren“ Boden und ein veränderungswilliges Umfeld treffen, wodurch die Programmwirkungen schneller bzw. stärker hervortreten können oder im Gegenteil auf großen Widerstand und Beharrungsvermögen stoßen, was die Wirksamkeit des Programms entsprechend negativ beeinflusst. Allerdings kann das Programm - wenn auch aufgrund der Größe und budgetären Ausstattung in eingeschränktem Maße - zu einer Veränderung der Rahmenbedingungen beitragen.

Das Programm Talente kann daher einen Beitrag zur Verbesserung der Nutzung des Humanpotentials und der Chancengleichheit im FTI-Bereich führen, eine spürbare Veränderung der Strukturen wird aber nur im Zusammenspiel mit anderen Maßnahmen erzielbar sein. 


\section{5 | Organisation}

Der Förderschwerpunkt Talente wurde im Jahr 2011 vom Bundesministerium für Klimaschutz, Umwelt, Energie, Mobilität, Innovation und Technologie (BMK) gestartet und wird von der Österreichischen Forschungsförderungsgesellschaft mbH (FFG) im Rahmen des Bereichs Strukturprogramme (SP) durchgeführt. Ein Rahmenvertrag regelt die Aufgaben und Berichtspflichten der FFG. In Austausch- und Abstimmungsmeetings werden zusätzliche Dinge besprochen, wie der Besuch von Messen oder um Werbekampagnen zu planen. Immer wieder gibt es Anfragen an die FFG z. B. bezüglich konkreter „Stories“ und Projekte, die zur Illustration dienen können.

Neben der Programmleitung, die seit Jahren unverändert ist, gibt es ein Programmmanagement für jede Programmlinie, wobei die MitarbeiterInnen flexibel in mehreren Linien mitarbeiten und es immer wieder zu Veränderungen in der Mitarbeit gekommen ist. Die Instrumente, die mit Ausnahme von FEMtech Forschungsprojekte keine Forschungsförderung im engeren Sinn darstellen, bedeuten neben den hohen Fallzahlen auch viel Beratungsaufwand für das Team. So sind die unmittelbaren Fördernehmerlnnen eher untypische FFG-Kundlnnen, z. B. setzen in den Unternehmen oft die Personalabteilung die Projekte um und nicht die mit der FFG vertraute F\&EAbteilung. Zudem werden andere Zielgruppen wie z. B. Jugendliche, Eltern, PädagogInnen, die keine Förderung bekommen, mitbetreut. Selbst bei den FEMtech Forschungsprojekten als klassische Forschungsförderung ist aufgrund der verpflichteten Genderrelevanz eine intensive Beratung nötig. Die Abwicklung des Programms beinhaltet daher viel Aufklärungsarbeit und diverse Begleitmaßnahmen (siehe Kap. 5.8). Neben den unterschiedlichen Bedürfnissen der Zielgruppen wird der enge Kundlnnenkontakt als sehr bereichernd empfunden und die meist sehr positiven Rückmeldungen der KundInnen (siehe unten). Aufgrund dieser Besonderheiten verwundert es auch nicht, dass die administrativen Kosten rd. $10 \%$ des Fördervolumens des Gesamtprogramms ausmachen und damit deutlich über denen in großvolumigen Programmen mit reiner Projektförderung liegen.

Der administrative Aufwand in den einzelnen Programmlinien variiert ebenso wie die Herausforderungen, die bei der Ansprache der Zielgruppe auftreten. Auch kommen entsprechend der Heterogenität der Instrumente im Programm verschiedene Ausschreibungs- und Auswahlverfahren zum Tragen, die von den AkteurInnen (BMK, FFG, GutachterInnen) überwiegend als adäquat beurteilt werden.

- Praktika für Schülerinnen und Schüler: Es erfolgt eine jährliche Ausschreibung bei laufender Einreichung. Die Praktika werden formal und inhaltlich geprüft und im Vier-Augen-Prinzip bewertet. Die Abwicklung birgt einige Herausforderungen. Oftmals sind die Personalabteilungen der Organisationen involviert und nicht die F\&E-Abteilung. Zusätzliche Zielgruppen, die gar keine Förderung bekommen, werden „,mitbetreut“, in diesem Fall die Schülerlnnen (und deren soziales Umfeld, wie Eltern etc.). Zudem werden Feedbackfragebögen an die FördernehmerInnen und PraktikantInnen ausgesandt und ausgewertet und es erfolgt ein vierzehntägiges Reporting an das BMK. Die spezielle Ansprache der Zielgruppen erfolgt über Multiplikatorlnnen, Jugend- und Mädchenzentren (z. B. Amazone) sowie Organisationen, in denen Päda- 
goginnen und Pädagogen vernetzt sind, etc. Die Werbung für SchülerInnenpraktika wird zeitweise automatisch in jedes FFG-E-Mail eingepflegt. Es gibt die Praktikabörse, jugendgerechte Videos und Texte finden sich auf der Webseite. PraktikumsplatzanbieterInnen werden jedes Jahr online gestellt, damit sich Schülerlnnen initiativ bewerben können.

- Talente regional: Es handelt sich um ein Wettbewerbsverfahren nach Ausschreibung, die im Herbst beginnt und für drei bis vier Monate geöffnet ist. Nach der Formalprüfung erfolgt die detaillierte Prüfung der Anträge und wirtschaftliche Aufbereitung sowie die Vorbereitung der Jury. Eine externe Jury bewertet die Anträge und reiht diese. In der Folge sind sehr viele Anfragen und Beratungsgespräche durchzuführen. Herausforderung in diesem Zusammenhang ist, dass die FörderwerberInnen nicht zur klassischen „FFG-Klientel“ gehören und keine Erfahrung mit dem e-Call-System haben. Die Ansprache der Zielgruppe und die Bewerbung der Ausschreibung erfolgt über eine Netzwerk- bzw. Informationsveranstaltung (siehe Kap. 5.8), wobei eine Mobilisierung hier kaum (mehr) erforderlich ist, da im Vorfeld schon viele Anfragen kommen. Hier erschweren die unregelmäßigen Ausschreibungen eine kontinuierliche Kundlnnenpflege.

- FEMtech Praktika für Studentinnen: In der Regel erfolgt eine jährliche Ausschreibung bei laufender Einreichung, 2019 gab es eine zweiteilige Einreichphase. Die Praktika werden formal und inhaltlich geprüft und im Vier-Augen-Prinzip bewertet. Auch hier werden Feedbackfragebögen an die FördernehmerInnen und Praktikantinnen ausgesandt und ausgewertet und es erfolgt ein monatliches Reporting.

- FEMtech Karriere / FEMtech Karriere-Check für KMU: Die Einreichung ist laufend möglich. Es erfolgt eine Beurteilung durch zwei externe GutachterInnen. Bei einem Patt wird ein drittes Gutachten eingeholt. Es gibt keine Jurysitzung, die Entscheidung fällt die FFG-Geschäftsführung. Bei FEMtech Karriere-Check wurden die Anträge anhand der im Ausschreibungsleitfaden angeführten Kriterien im Vier-Augen-Prinzip FFG-intern begutachtet. Die Programmlinie ist äußerst beratungsintensiv, es gibt Fälle wo Organisationen im Vorfeld über ein Jahr beraten wurden. Bei FEMtech Karriere ist die Akquisetätigkeit essentiell, um FördernehmerInnen zu gewinnen. So werden verschiedenste Veranstaltungen für die Informationsverbreitung genützt, allen voran das FEMtech Netzwerktreffen. Es gibt laufende Kontakte zu GenderexpertInnen. Im August bis November 2015 und im November 2016 gab es zudem intensive Mobilisierungsphasen, wo gezielt FördernehmerInnen aus anderen Programmlinien telefonisch über FEMtech Karriere informiert wurden, für solche Aktivitäten sind aber die Ressourcen begrenzt.

- FEMtech Forschungsprojekte: Es handelt sich um ein Wettbewerbsverfahren nach Ausschreibung. Pro Antrag werden zwei Fachgutachten eingeholt und anschließend in einer Jurysitzung die Anträge bewertet und gereiht. Die Ausschreibung erfolgt ebenfalls im Herbst und ist drei bis vier Monate geöffnet. Die Ansprache der Zielgruppe und die Bewerbung der Ausschreibung erfolgt $u$. a. über eine Informationsveranstaltung (siehe Kap. 5.8). Die Beratungen gestalten sich auch hier als sehr intensiv, da die Genderrelevanz der Projekte ein K.o.-Kriterium darstellt und der Beratungsaufwand deshalb selbst bei bestehenden Kundlnnen höher ist. 
- Karriere-Grants: Die Einreichung ist laufend möglich. Die Bewertung erfolgt im Vier-AugenPrinzip. Die Ansprache der EinzelforscherInnen gestaltet sich als herausfordernd, da der Zeitpunkt für die Förderung für die/den Einzelne/n nur punktuell relevant ist. Informationen über die Förderung gibt es auf der Plattform Euraxess. Ebenso werden Unternehmen und Forschungseinrichtungen dahingehend beraten, die Informationen an potentielle BewerberInnen weiterzugeben, z. B. auf Karrieremessen. Universitäten kennen die Förderung in der Regel bereits und informieren die Bewerberlnnen im Zuge des Bewerbungsprozesses entsprechend.

- Jobbörse: Die Hauptaufgabe der FFG in Hinblick auf die Jobbörse ist die Qualitätskontrolle der gelieferten Daten. Hier wird seit Mitte 2020 einmal im Monat anhand einer Stichprobe die Vollständigkeit überprüft und einmal im Quartal die Qualität der Anzeigen (hinsichtlich Zuordnung etc.). Hinzu kommt die Abstimmung mit den externen DienstleisterInnen (DatenlieferantIn und BetreiberIn der FFG-Website) und das Vergabeverfahren (alle 3 Jahre). Laufend werden Unternehmen betreut, die ihr Inserat auf der Jobbörse platzieren wollen. Im Jahr 2019 erfolgte eine PR-Offensive, um mehr Jobsuchende zu gewinnen und auch Unternehmen, um sich für die Jobbörse registrieren zu lassen. Auch wurden die Alumni-Klubs der Universitäten gewonnen, auf die Jobbörse zu verlinken.

Für die FördernehmerInnen stellen sich die Verfahren, insbesondere bei den Kleinstförderungen, als sehr einfach dar. So schätzen diese die schnelle, einfache und unkomplizierte Abwicklung der Förderung von Praktika für SchülerInnen. Durch das Online-Portal wird der administrative Aufwand für die FördernehmerInnen auf ein Minimum beschränkt. Die FördernehmerInnen müssen bei der Erstellung des Förderantrags keine komplizierten Formulare ausfüllen. Das Verhältnis zwischen Formalaufwand und Förderleistung wird im Jahr 2019 (ähnlich wie in den Jahren davor) von $39 \%$ als sehr ausgewogen und von $57 \%$ als ausgewogen beschrieben.

Der administrative Aufwand beschränkt sich auf ein sehr angenehmes Minimum.

Die Tatsache, dass ich alles online in einer einheitlichen Plattform abwickeln kann, ganz ohne komplizierte Formulare ausfüllen zu müssen.

Unkomplizierte Förderung mit nur sehr geringem organisatorischem Aufwand für das Unternehmen.

Bei der Abwicklung der FEMtech Praktika für Studentinnen zeigen sich ein ähnliches Bild und sehr zufriedene Kundlnnen. Das Verhältnis zwischen Aufwand und Förderhöhe wird von 99 \% als (sehr) ausgewogen beschrieben. Die Antragstellung und die Abwicklung der Förderung gestaltet sich für die teilnehmenden Organisationen einfach und auch die rasche Förderabwicklung wird gelobt.

Der einfache, relativ unbürokratische Ablauf und der geringe Zeitaufwand bei der Antragstellung und die schnelle Abwicklung des Antrags sind besonders hervorzuheben.

Die Einfachheit der Abwicklung hilft, dass man diese Anträge gerne einreicht. 
Und auch die Karriere-Grants-FördernehmerInnen zeigen sich mit der Abwicklung zufrieden. Die Zufriedenheit mit den verschiedensten Aspekten im Rahmen der Programmabwicklung ist unter den EmpfängerInnen der Interview-Grants sehr hoch (siehe Grafik 1). Mehr als $80 \%$ der Befragten sind mit der Verfügbarkeit von Informationen und der Betreuung und Unterstützungsleistungen durch die FFG während der Projektlaufzeit (sehr) zufrieden, ebenso wie mit dem Aufwand für die Antragsstellung und Berichterstattung im Verhältnis zum Nutzen durch die Förderung - wobei sich rd. zwei Drittel sehr zufrieden zeigen. Auch bezüglich der Höhe der Förderung und der förderbaren Kostenarten ist die Zufriedenheit hoch, allerdings sind "nur" rd. die Hälfte der Befragten sehr zufrieden und (knapp) jeder Zehnte eher unzufrieden.

Grafik 1 | Zufriedenheit der befragten Interview Grants EmpfängerInnen mit der Förderung bzw. Abwicklung der Förderung, in Prozent

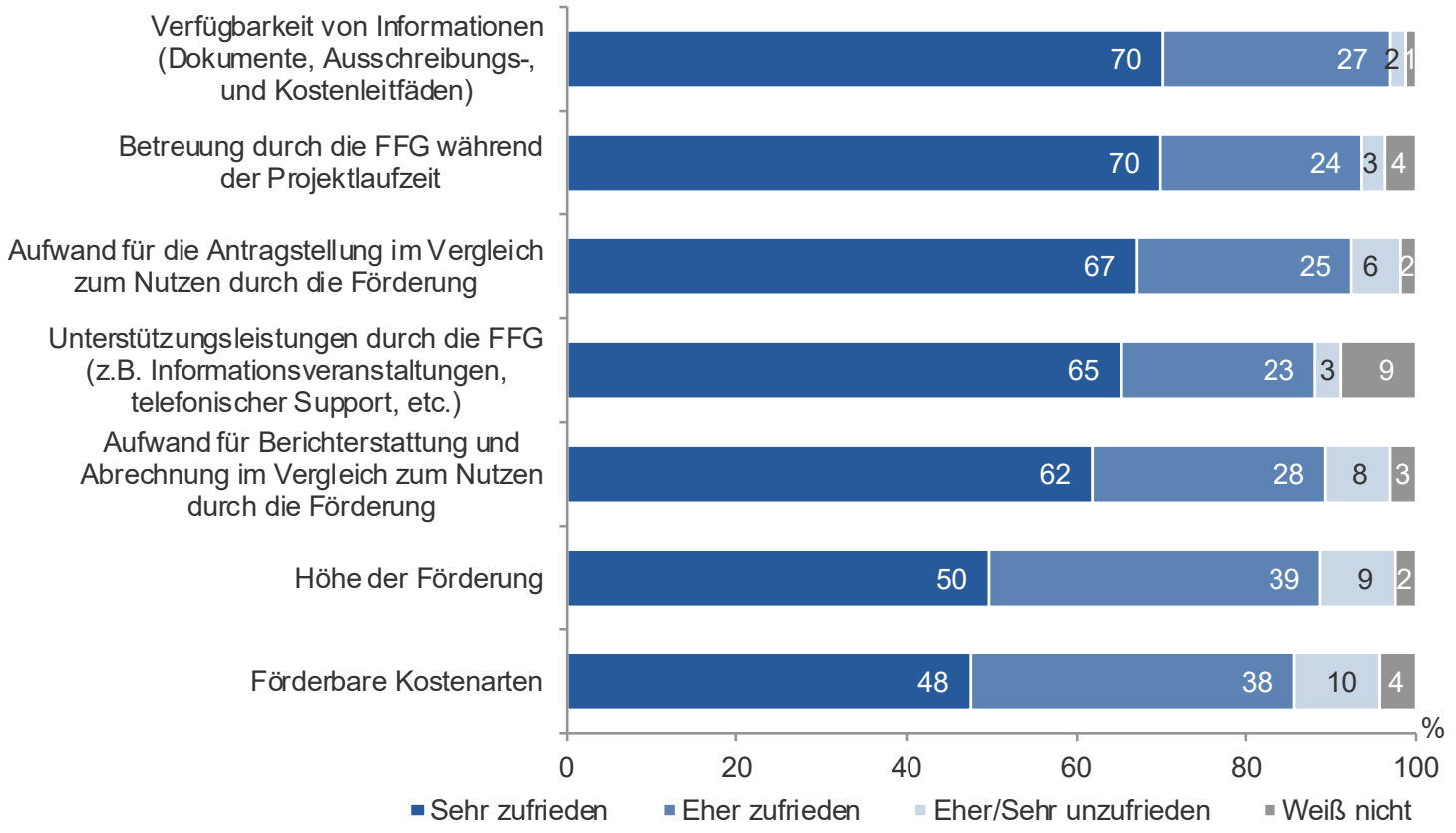

Quelle: Befragung Karriere-Grants Fördernehmerlnnen, KMU Forschung Austria; n=160-161

Die EmpfängerInnen der Relocation-Grants zeigen sich noch zufriedener in Hinblick auf die Abwicklung, wobei die Anteile der sehr Zufriedenen noch größer sind und (mehr als) $90 \%$ mit den meisten Aspekten der Abwicklung (eher) zufrieden sind (siehe Grafik 2). Ein ähnliches Bild zeigt sich bei den Personen, die einen Dual Career Grant in Anspruch genommen haben (siehe Grafik 3). Hier gibt es lediglich in Hinblick auf die förderbaren Kostenarten mit $14 \%$ einen etwas höheren Anteil an Befragten, die (eher) unzufrieden damit sind. 
Grafik 2 | Zufriedenheit der befragten Relocation Grants Empfängerlnnen mit der Förderung bzw. Abwicklung der Förderung, in Prozent

Aufwand für die Antragstellung im Vergleich zum Nutzen durch die Förderung

Betreuung durch die FFG während der Projektlaufzeit

Unterstützungsleistungen durch die FFG (z.B. Informationsveranstaltungen, telefonischer Support, etc.)

Verfügbarkeit von Informationen (Dokumente, Ausschreibungs-, und Kostenleitfäden)

Aufwand für Berichterstattung und Abrechnung im Vergleich zum Nutzen durch die Förderung

Höhe der Förderung

Förderbare Kostenarten

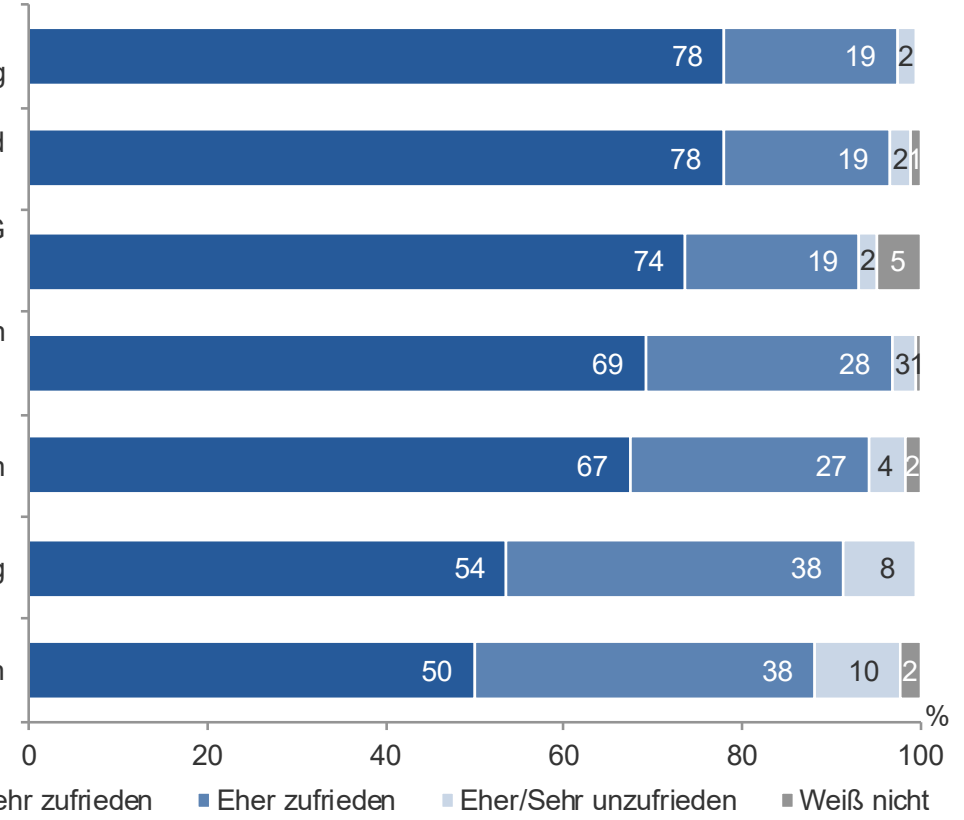

Quelle: Befragung Karriere-Grants Fördernehmerlnnen, KMU Forschung Austria; n=496-498

Grafik 3 | Zufriedenheit der befragten Dual Career Grants EmpfängerInnen mit der Förderung bzw. Abwicklung der Förderung, in Prozent

Aufwand für die Antragstellung im Vergleich zum Nutzen durch die Förderung

Betreuung durch die FFG während der Projektlaufzeit

Aufwand für die Berichterstattung und Abrechnung im Vergleich zum Nutzen durch die Förderung

Verfügbarkeit von Informationen (Dokumente, Ausschreibungs-, und Kostenleitfäden)

Unterstützungsleistungen durch die FFG

(z.B. Informationsveranstaltungen, telefonischer Support, etc.)

\section{Höhe der Förderung}

Förderbare Kostenarten

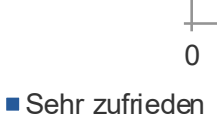

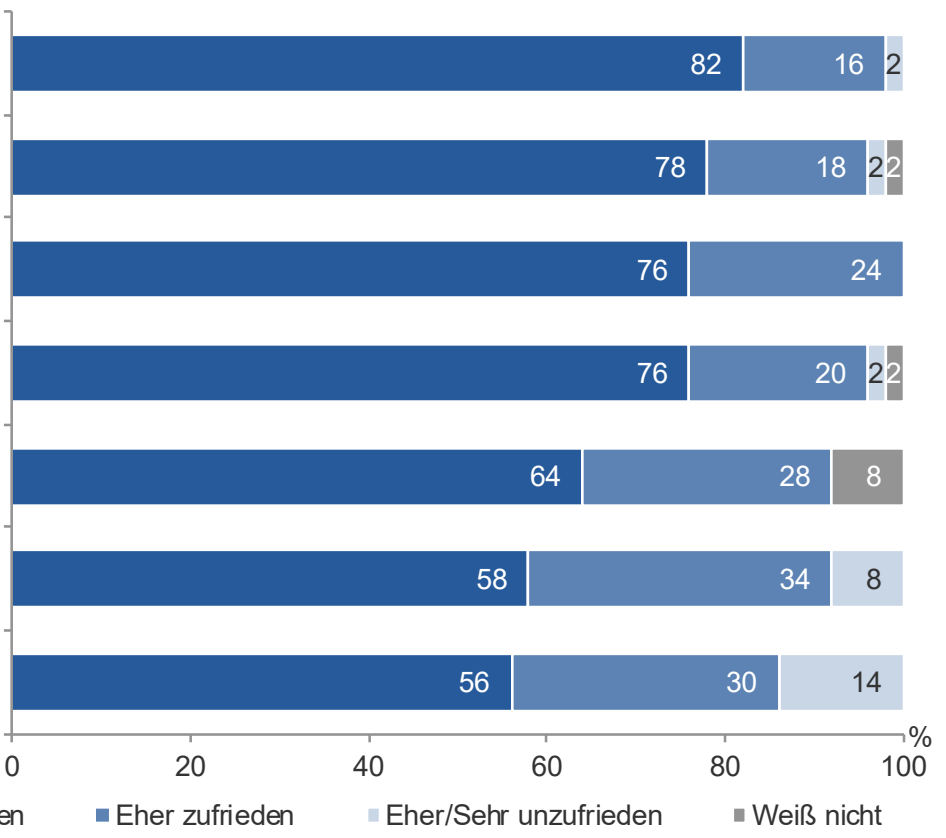

Quelle: Befragung Karriere-Grants FördernehmerInnen, KMU Forschung Austria; n=50 
Aber auch bei den komplexeren Programmlinien wie Talente regional gibt es überwiegend positive Resonanz in Hinblick auf die Programmabwicklung und die laufende Optimierung der Ausgestaltung. Von Seiten der befragten ExpertInnen wird beispielsweise die Verlängerung der Projektlaufzeit auf 24 bis 36 Monate ab dem Jahr 2015 begrüßt, um mehr Kontinuität bei der Abwicklung zu gewährleisten. Bei der Befragung der Projektteilnehmerlnnen durch das ibw im Rahmen des Review Talente regional (2018) zeigt sich eine hohe Zufriedenheit in Hinblick auf die Gestaltung der Ausschreibung und die konkrete Abwicklung der Projekte. Mindestens $85 \%$ sind mit den verschiedenen Vorgaben der Ausschreibung und Projektabwicklung zufrieden. Nach Meinung der Befragten ist die formelle Abwicklung der Projekte im Zeitverlauf einfacher geworden, jedoch ist die Komplexität der zu berücksichtigenden Aspekte durch die Hinzunahme der Gender- und Migrationsthematik gestiegen. Die PädagogInnen sind hier verunsichert, wie sie diese Aspekte integrieren sollen, da teilweise projektspezifische Anknüpfungspunkte fehlen.

Auch in Bezug auf die Abwicklung von FEMtech Karriere Projekten bzw. des FEMtech KarriereChecks für KMU ist die Zufriedenheit mit der Förderabwicklung hoch (siehe Grafik 4). Die befragten Projektteilnehmerlnnen sind mit der Verfügbarkeit von Informationen, wie Dokumenten, Ausschreibungs- und Kostenleitfäden, sowie mit der Laufzeit der Projekte von 6 bis 24 Monate bzw. 12 Monate im Falle des FEMtech Karriere-Checks für KMU sehr zufrieden. Auch die Unterstützungsleistungen durch die FFG bei Informationsveranstaltungen, der telefonische Support und die Betreuung während der Projektlaufzeit werden sehr geschätzt. Die zeitlichen Aspekte von der Einreichung bis zum Vertrag, die Auswahl- und Bewertungskriterien sowie die förderbaren Kostenarten werden ebenfalls positiv beurteilt. Auch in Hinblick auf den Aufwand für die Antragstellung, Berichterstattung und Abrechnung im Vergleich zum Nutzen sowie bezüglich der Höhe der Förderung herrscht mehrheitlich eine große Zufriedenheit. Lediglich die Möglichkeit für Änderungen während der Projektlaufzeit lässt sich für viele nicht beurteilen, da sie nicht davon Gebrauch machen mussten. 
Grafik 4 | Zufriedenheit mit verschiedenen Aspekten der Förderung und Förderabwicklung von FEMtech Karriere und des FEMtech Karriere-Checks für KMU, Anzahl der befragten Organisationen

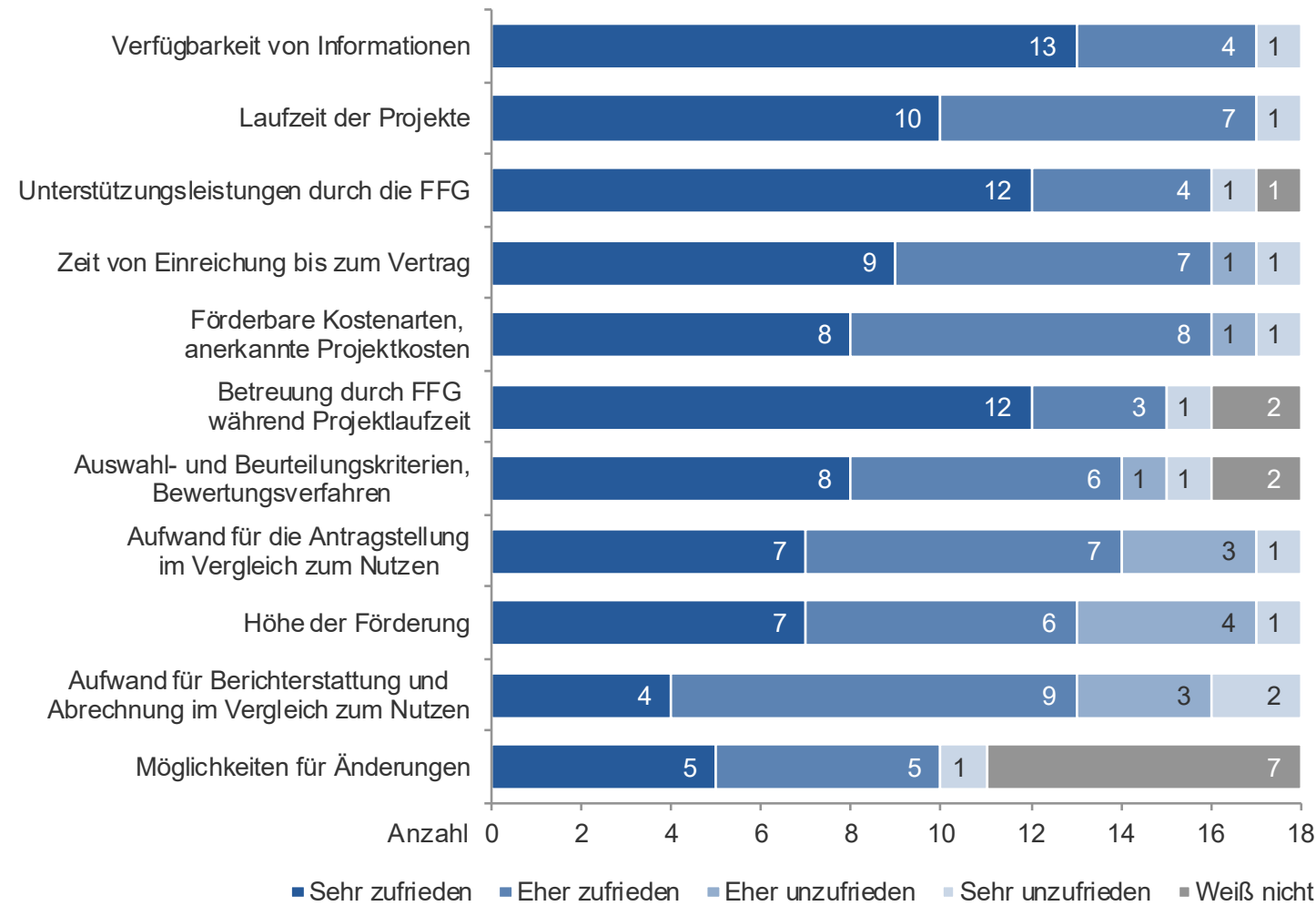

Quelle: Befragung FEMtech Karriere 2020, KMU Forschung Austria, n=18

Auch im Rahmen der Fallstudien ist die Zufriedenheit mit der Abwicklung der FEMtech Karriere Projekte und der Betreuung durch die FFG ersichtlich. Lediglich die Vorgaben für die Module werden eher verkomplizierend erlebt und könnten daher noch weiter vereinfacht werden.

In der Programmschiene war es sehr flott im Vergleich zu anderen Förderungen.

Über den e-Call kann man immer schnell Fragen stellen und bekommt schnell eine Rückmeldung.

Der Antrag war im Vergleich zu anderen Förderungen, größeren Projekten relativ unaufwändig auch in der Abwicklung, es war ja kein Zwischenbericht erforderlich, nur der Endbericht.

Die Anforderungen waren klar verständlich. Bei den Modulen könnte man die Anforderungen etwas reduzieren. 


\section{6 | Die Programmlinien}

Im Folgenden wird auf die einzelnen Programmlinien des Förderschwerpunkts Talente im Detail eingegangen. Neben einer kurzen Darstellung der Anforderungen an die Projekte wird ein Überblick über die Struktur der geförderten Projekte und deren Wirkungen im Zeitraum von 2011 bis 2019 gegeben.

\section{1| Praktika für Schülerinnen und Schüler}

Mit Hilfe der Programmlinie Praktika für Schülerinnen und Schüler gilt es, Talente zu entdecken und zu entwickeln. Die Praktika ermöglichen Jugendlichen ab 15 Jahren erste Praxiserfahrungen im naturwissenschaftlich-technischen Bereich zu sammeln und bieten Unternehmen, Universitäten, Fachhochschulen, außeruniversitären Forschungseinrichtungen, etc. die Möglichkeit, junge Menschen zu fördern und frühzeitig an ihre Organisation zu binden.

Tabelle 1 | Eckpunkte der Praktika für Schülerinnen und Schüler

\begin{tabular}{|c|c|}
\hline $\begin{array}{l}\text { Praktika für Schü- } \\
\text { lerlnnen }\end{array}$ & Kurzbeschreibung \\
\hline Ziele & Junge Menschen für Forschung und Entwicklung zu begeistern \\
\hline Zielgruppen & $\begin{array}{l}\text { Unternehmen, Universitäten, Fachhochschulen, außeruniversitäre Forschungsein- } \\
\text { richtungen, Technologietransfer-Einrichtungen/Innovationsmittlerlnnen etc. und } \\
\text { sonstige nicht-wirtschaftliche Einrichtungen (Fördernehmerlnnen) } \\
\text { Schülerlnnen, die mindestens } 15 \text { Jahre alt sind und eine österreichische Schule } \\
\text { besuchen. }\end{array}$ \\
\hline $\begin{array}{l}\text { Quotenregelun- } \\
\text { gen }\end{array}$ & $\begin{array}{l}\text { 2012-2014: In allen Anträgen mit } 3 \text { oder mehr Praktika muss zumindest ein Drittel } \\
\text { der Praktikumsplätze an Mädchen vergeben werden (2012: nur in Großunterneh- } \\
\text { men). } \\
\text { 2015-2019: Mindestens } 50 \% \text { der Praktika in einem Antrag müssen an Schülerln- } \\
\text { nen nicht-technischer Schulen vergeben werden }\end{array}$ \\
\hline Inhalt & $\begin{array}{l}\text { Praktika für Jugendliche im FTI-Bereich, mit naturwissenschaftlichem oder techni- } \\
\text { schem Bezug im Zeitraum von Juni bis September. Diese dienen als Bildungs- und } \\
\text { Orientierungsangebot sowie als Impulsgeber für eine entsprechende Studien- oder } \\
\text { Berufswahl. }\end{array}$ \\
\hline Einreichverfahren & $\begin{array}{l}\text { Laufende Einreichung nach Ausschreibung (Jänner bis Juli 2011, 2012, } 2013 \text {, } \\
\text { 2014, 2015, 2016, 2017, 2018, 2019), bei Mittelausschöpfung vorzeitiges Schließen } \\
\text { der Ausschreibung }\end{array}$ \\
\hline Laufzeit & $\begin{array}{l}\text { Praktikumsdauer von mindestens } 4 \text { Wochen (mind. } 26 \text { Kalendertage) mit mind. 28,5 } \\
\text { Wochenstunden }\end{array}$ \\
\hline Förderhöhe & $\begin{array}{l}1.000 € \text { pro Praktikum (davon mindestens } € 700 \text {,- Praktikumsentgelt ( } 2011 \text { bis } \\
2017 \text { ) } \\
1.200 € \text { pro Praktikum (davon mindestens } € 750 \text {,- Praktikumsentgelt) (2018 und } \\
2019 \text { ) }\end{array}$ \\
\hline Spezifika & 2013 und 2014: Themenschwerpunkt Mobilität der Zukunft \\
\hline
\end{tabular}




\subsection{1 | Struktur der geförderten Praktikumsplätze und Organisationen}

Im Zeitraum von 2011 bis 2019 wurden insgesamt 12.911 geförderte Praktikumsplätze für Schülerinnen und Schüler im Bereich von naturwissenschaftlichen und technischen FTI-Aktivitäten zur Verfügung gestellt. Die meisten Praktika, nämlich 1.710, wurden im Jahr 2014 gefördert. Im Jahr 2018 konnten trotz der Anpassung der Förderungshöhe (€1.200,- statt $€ 1.000$,- pro Praktikum) aufgrund höherer Fördermittel als üblich gleich viele Praktika wie im Jahr 2017 gefördert werden. Im Jahr 2019 wurden 1.270 Praktikumsplätze finanziell unterstützt.

Grafik 5 | Anzahl der geförderten Praktika für Schülerinnen und Schüler, Ausschreibungen 2011-2019

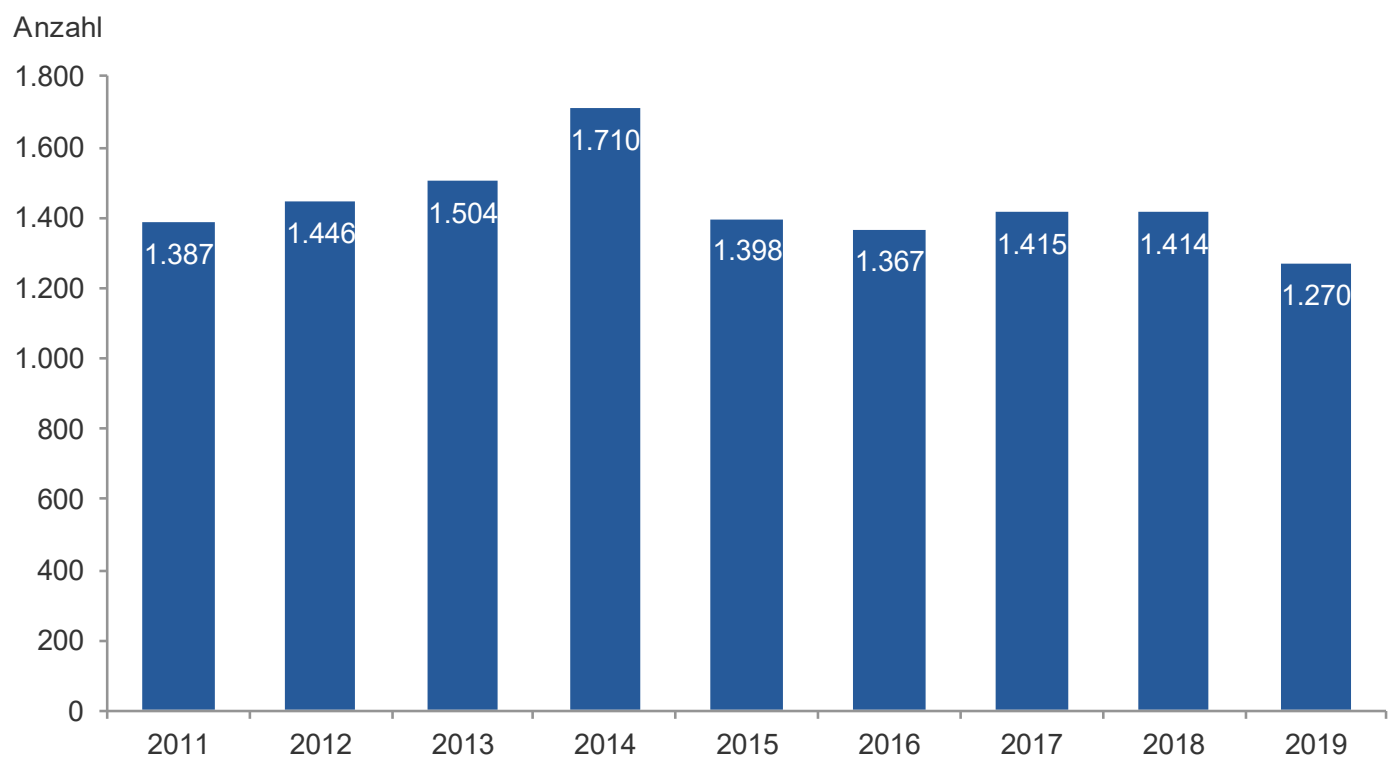

Quelle: FFG; Berechnung und Darstellung KMU Forschung Austria

Inhaltlich konzentrieren sich die Praktika für Schülerinnen und Schüler auf den Bereich der technischen Wissenschaften, wie Grafik 6 zeigt. Durchschnittlich entfielen 58 \% der Praktika im Zeitraum von 2013 bis 2019 auf diese Wissenschaftsdisziplin und $35 \%$ der Praktika konzentrierten sich auf Naturwissenschaften. Seit 2013 haben die technischen Wissenschaften als Praktikumsfokus jedoch etwas an Bedeutung verloren (von 64 \% 2013 auf 52 \% 2019). Dafür werden verstärkt Praktika im Bereich der Naturwissenschaften (von 32 \% 2013 auf 37 \% 2019), aber auch in der Humanmedizin (von $2 \% 2013$ auf 7 \% 2019) umgesetzt.

Auch bei den Themenfeldern der SchülerInnenpraktika zeigen sich Verschiebungen der Prioritäten. Während Praktika im Bereich von IKT und Produktion mit jeweils $27 \%$ der Praktikumsplätze im Jahr 2011 vor allem im Produktionsbereich (13\% 2019), aber auch bei den Informations- und Kommunikationstechnologien (22\% 2019) im Zeitverlauf etwas weniger gefragt sind, gewinnen Praktika im Bereich der Life Sciences und in sonstigen Themenfeldern an Relevanz. Im gesamten Zeitraum von 2011 bis 2019 stehen bei den Praktika für Schülerinnen und Schüler neben sonstigen Themenbereichen IKT, Produktion und Life Sciences im Vordergrund. 
Grafik 6 | Wissenschaftsdisziplinen und Themenfelder der geförderten Praktika für Schülerinnen und Schüler Ausschreibungen 2011-2019, in Prozent

Wissenschaftsdisziplinen 2013-19

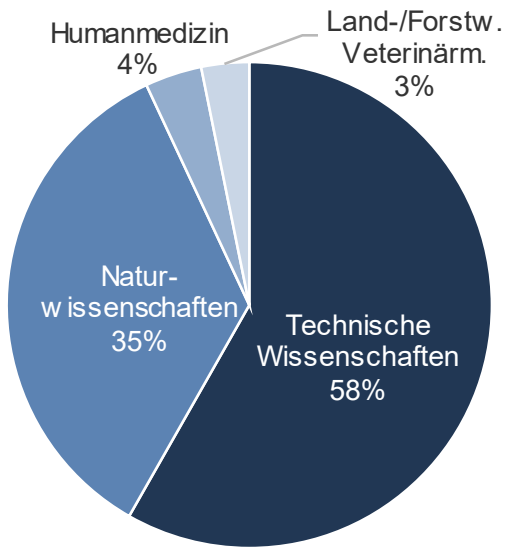

Themen der Praktika 2011-2019

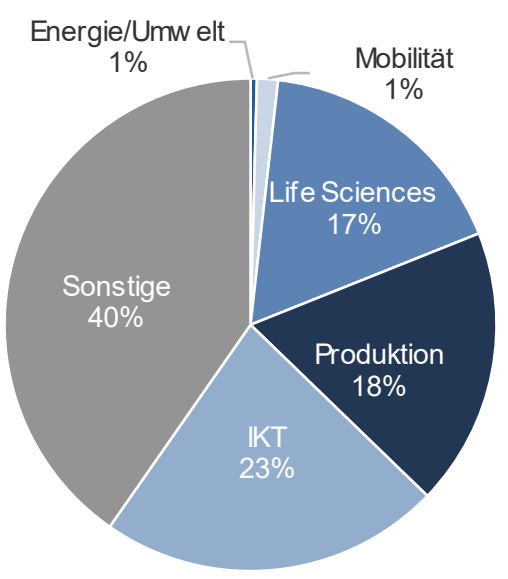

Quelle: FFG - Talente SchülerInnenpraktika Schlussauswertungen 2013-2019, FFG; Berechnung und Darstellung KMU Forschung Austria

Nicht nur thematische Schwerpunkte, sondern auch regionale Unterschiede sind zu beobachten. Mehr als ein Fünftel der geförderten Praktikumsplätze werden in den Jahren 2011 bis 2019 in Wien (23\%) und in der Steiermark (22\%) angeboten. Lediglich ein Bruchteil der Praktika für Schülerinnen und Schüler wird hingegen im Burgenland (1\%), Vorarlberg (2\%) oder Salzburg (3\%) realisiert. Im Zeitverlauf gewinnen vor allem Praktika in der Bundeshauptstadt weiter an Relevanz: 2011 wurden 21 \% der SchülerInnenpraktika in Wien umgesetzt, 2019 traf dies bereits auf $28 \%$ der Praktikumsplätze zu.

Grafik 7 | Geförderte Praktika für Schülerinnen und Schüler nach Bundesländern, Ausschreibungen 20112019, in Prozent

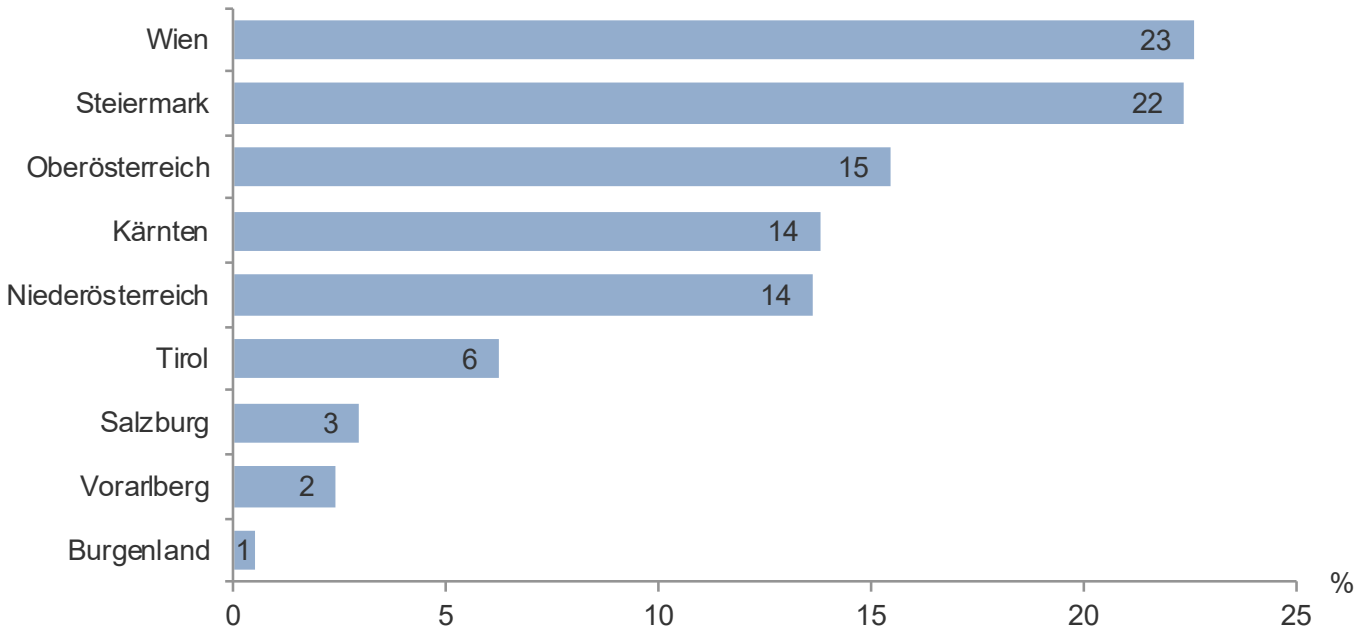

Quelle: FFG; Berechnung und Darstellung KMU Forschung Austria 
Von den 12.911 zwischen 2011 und 2019 geförderten Praktikumsplätzen wurden 52 \% in Unternehmen und $32 \%$ in hochschulischen Einrichtungen angeboten (siehe Grafik 8). Weitere $11 \%$ der Praktika fanden in außeruniversitären Einrichtungen und $4 \%$ in Kompetenzzentren, kooperativen Forschungseinrichtungen, Zentren und Clustern statt. Praktika, die in Unternehmen absolviert werden, finden vorwiegend in Großunternehmen (27\%) sowie in Kleinunternehmen (18\%) statt. Im Zeitverlauf hat sich vor allem die Anzahl der Praktikumsplätze in Unternehmen (von 57 \% 2011 auf 41 \% 2019) reduziert. Gleichzeitig ist das Angebot an Praktika für Schülerinnen und Schüler im universitären Bereich (von 26 \% 2011 auf 40 \% 2019) gestiegen.

Grafik 8 | Praktikumsplätze nach Organisationsarten, Ausschreibungen 2011-2019, in Prozent

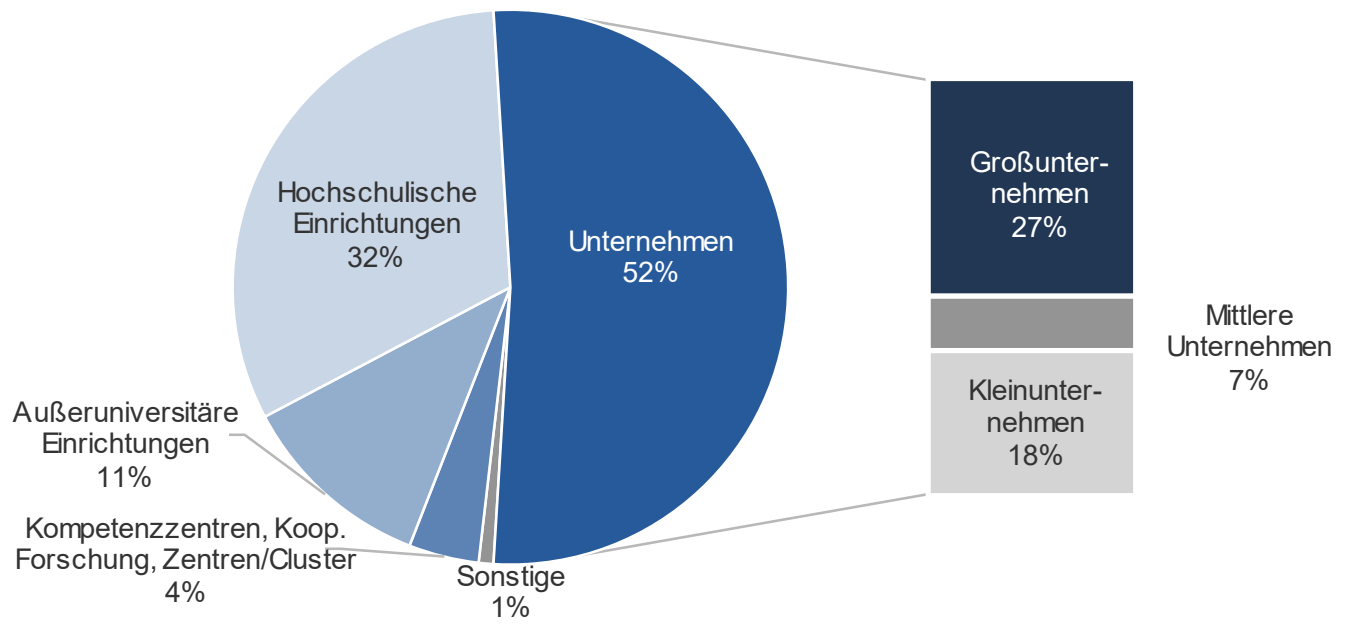

Quelle: FFG; Berechnung und Darstellung KMU Forschung Austria

Die Unternehmen, Hochschulen und Forschungseinrichtungen bieten oftmals mehrere Praktikumsplätze für Schülerinnen und Schüler an. Die Analyse der teilnehmenden Organisationen ohne Berücksichtigung der Anzahl der jeweils angebotenen Praktikumsplätze im Jahr 2019 zeigt beispielsweise, dass 328 Organisationen insgesamt 1.270 geförderte Praktika zur Verfügung stellen. Dabei waren es in erster Linie Unternehmen (46\%), die Praktika für Schülerinnen und Schüler offerierten, gefolgt von Universitäten (36\%). Außeruniversitäre Einrichtungen stellten im Jahr 20199 \% der PraktikumsanbieterInnen.

Im Jahr 2019 bot etwa ein Viertel (25\%) der 328 Organisationen einen Praktikumsplatz für Schülerlnnen an und rd. ein Drittel (32\%) stellte zwei Praktikumsmöglichkeiten zur Verfügung, wie Grafik 9 zeigt. Ein knappes Fünftel (19\%) offerierte für 3 oder 4 SchülerInnen eine Praktikumsoption. $6 \%$ aller beteiligten Organisationen stellten 2019 sogar mehr als 10 geförderte Praktikumsplätze für SchülerInnen zur Verfügung. Zwischen Unternehmen und Hochschulen sind hinsichtlich der Anzahl der Praktikumsplätze, die sie vergeben, kaum Unterschiede zu beobachten. Dabei ist jedoch zu berücksichtigen, dass nicht die jeweiligen Universitäten insgesamt, sondern die verschiedenen Universitätsinstitute als eigene Organisationen erfasst werden. Bei den Forschungseinrichtungen besteht eine leichte Tendenz, gleichzeitig etwas mehr PraktikantInnen aufzunehmen. Im Jahr 2019 bot beispielsweise ein Fünftel der Forschungseinrichtungen (20\%) 
mehr als 8 Praktika an (im Vergleich zu $11 \%$ insgesamt). Gleichzeitig ist der Anteil der Forschungseinrichtungen mit nur ein oder zwei Praktikumsplätzen etwas niedriger als bei allen Organisationen insgesamt (39\% vs. $57 \%$ ).

Im Zeitraum von 2011 bis 2019 stellten die Top-10 Praktikumsanbieterlnnen zwischen $17 \%$ und 27 \% der Praktikumsplätze zur Verfügung. Besonders in den Jahren 2013 und 2014 und damit vor der Einführung der Quotenregelung für SchülerInnen nicht-technischer Schulen war die Konzentration auf einzelne AnbieterInnen besonders stark ausgeprägt. Die Höchstzahlen an geförderten Praktika der Top1-AnbieterInnen lagen in den Jahren 2011 bis 2019 zwischen 41 und 169 Praktikumsplätzen für SchülerInnen. Unter den Top-10 AnbieterInnen sind sowohl Unternehmen als auch Universitätsinstitute und Fachhochschulen sowie Forschungseinrichtungen vertreten.

Grafik 9 | Anzahl der Praktika pro Organisation im Jahr 2019 und Anteile der Top-10 Anbieterlnnen an allen Praktikumsplätzen, Ausschreibungen 2011 - 2019, in Prozent

\section{Anzahl der Praktika pro Organisation, 2019}

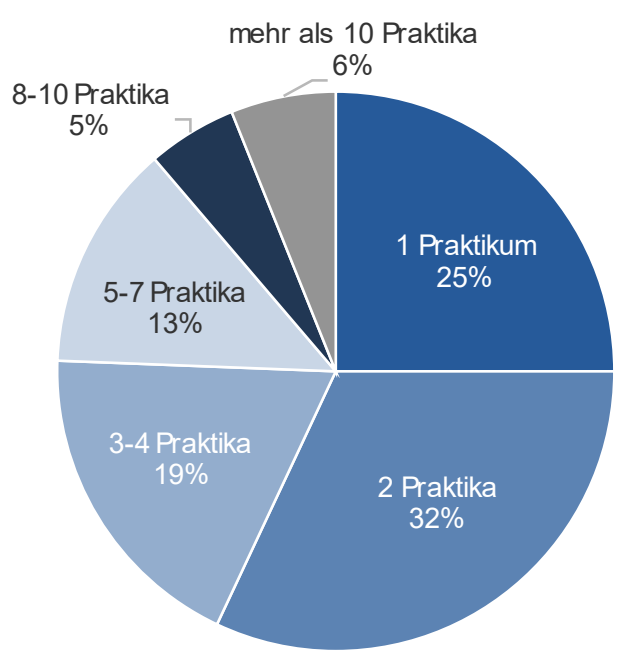

Anteil Top-10 AnbieterInnen

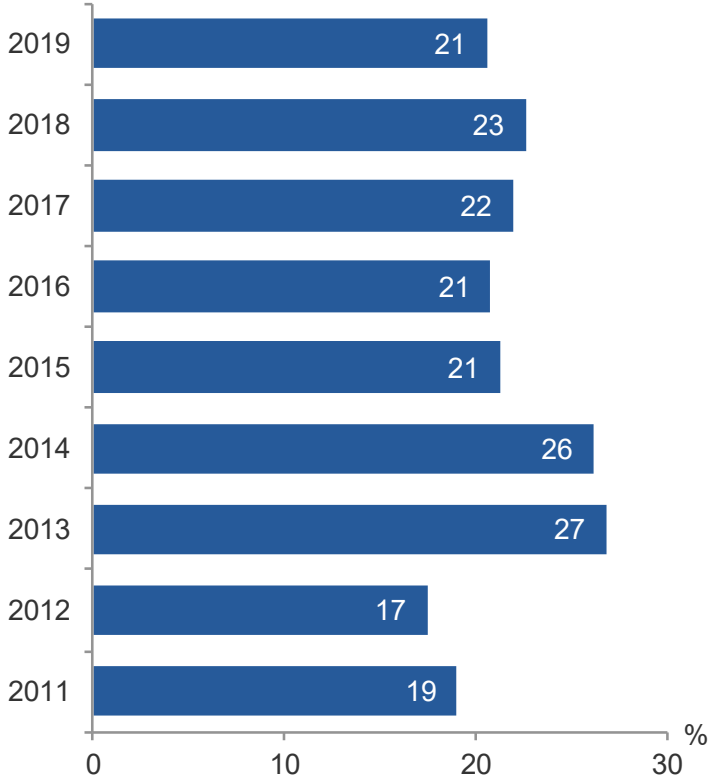

Quelle: FFG; Talente SchülerInnenpraktika Schlussauswertungen 2011-2019

In Hinblick auf die Inanspruchnahme der geförderten Praktika von Seiten der Schulen zeigt sich, dass SchülerInnen von 402 Schulen im Jahr 2019 diese Möglichkeit zum Sammeln beruflicher Praxis genutzt haben. Bei $43 \%$ der Schulen nahm nur ein/e Schülerln diese Praktikumsoption wahr und bei jeweils rd. einem Fünftel waren 2 SchülerInnen bzw. 3 bis 4 Jugendliche in Praktika involviert. Lediglich bei $4 \%$ der Schulen nutzten mehr als 10 Schülerlnnen das Förderangebot. Zu den Schulen, bei denen mehr als 10 Jugendliche ein gefördertes Praktikum erhielten, zählten in erster Linie Schulstandorte mit einer großen Schülerlnnenanzahl - somit hauptsächlich höhere 
technische Lehranstalten (HTL) oder berufsbildende höhere Schulen mit Schwerpunkt auf chemische Industrie, Umwelt oder Landwirtschaft, aber auch Gymnasien.

Von Seiten der SchülerInnen ist die Mehrfachnutzung des geförderten Praktikumsangebots nicht stark ausgeprägt. Die überwiegende Mehrheit (85 \%) hat im Zeitraum von 2011 bis 2019 nur ein gefördertes Praktikum absolviert. $12 \%$ nahmen zwei geförderte Praktika in Anspruch und $3 \%$ machten dreimal oder noch öfter von dieser Fördermöglichkeit Gebrauch.

Grafik 10 | Praktikumsnutzung in den Schulen: Anteil der involvierten Schulen 2019 und Anteil der SchülerInnen nach der Anzahl der Praktika, Ausschreibungen 2011-2019, in Prozent

\section{Anzahl der Praktika pro Schule,} 2019

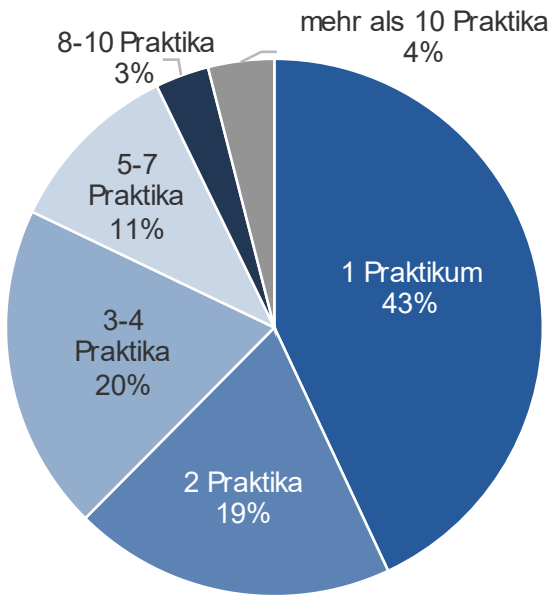

\section{Praktikumsnutzung pro Schülerln 2011-2019}

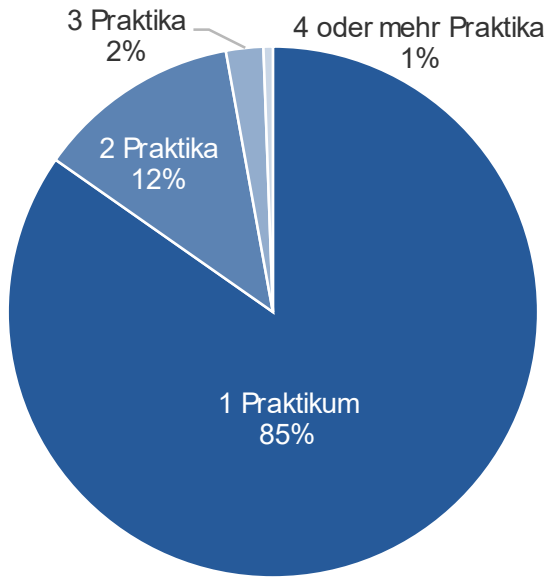

Quelle: FFG; Berechnung und Darstellung KMU Forschung Austria

Der Großteil der Praktikumsplätze wird aufgrund der Eigeninitiative der teilnehmenden Organisationen vergeben. Die überwiegende Mehrheit der FördernehmerInnen hat die SchülerInnen für die geförderten Praktika ( 80 \% bis 87 \%) im Zeitraum von 2012 bis 2019 selbst rekrutiert. Die Praktikumsbörse gewinnt im Zeitverlauf jedoch immer mehr an Bekanntheit und Beliebtheit (siehe Grafik 11). Im Jahr 2019 wurde bereits ein Fünftel der Praktikumsplätze mit Hilfe der Börse vergeben. 
Grafik 11 | Nutzung der Praktikumsbörse, Ausschreibungen 2012-2019, in Prozent

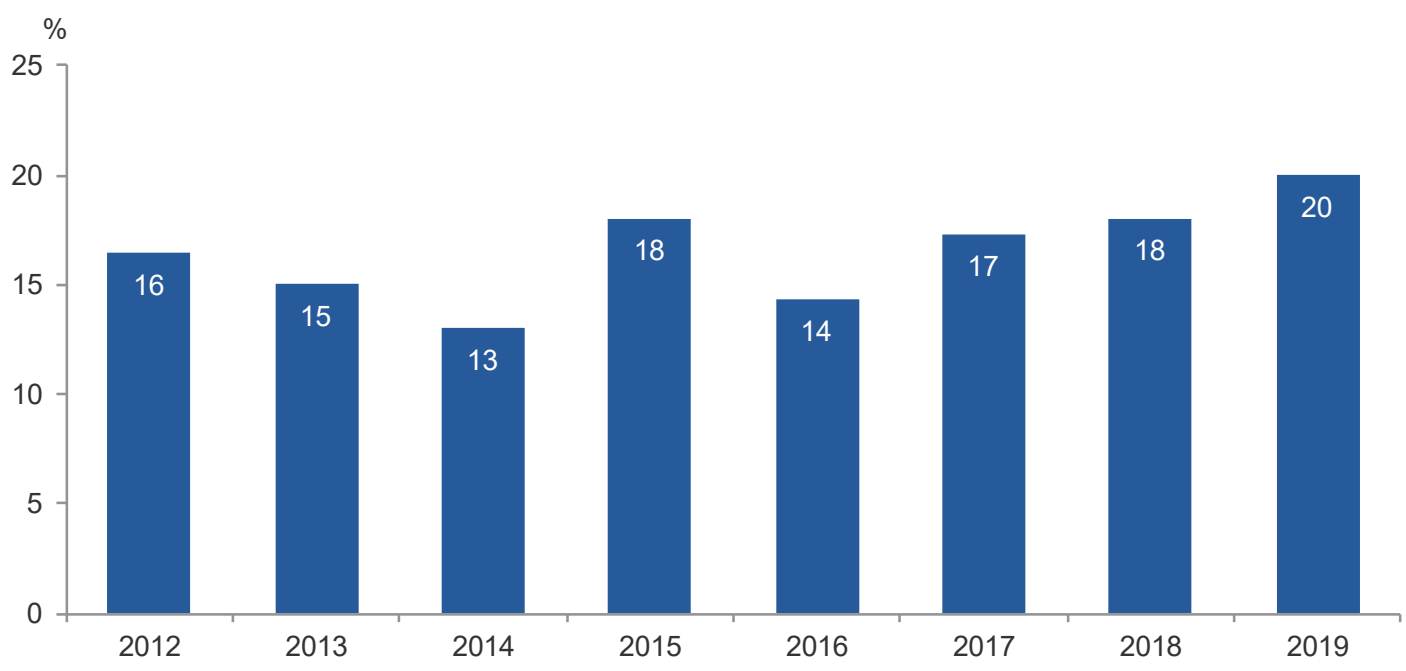

Quelle: FFG - Talente SchülerInnenpraktika Schlussauswertungen 2011-2019

Die Praktikumsbörse wird vorwiegend von AHS-Schülerlnnen genutzt, wobei im Zeitraum von 2015 bis 2019 zwischen 54 \% und 61 \% davon Gebrauch machten. Da AHS-Schülerlnnen über weniger Wirtschafts- und Unternehmenskontakte verfügen, ist das Angebot der Börse eine wichtige Unterstützung für die Akquise von Praktikumsplätzen.

Die Fördernehmerlnnen schätzen die Unterstützung durch die Praktikumsbörse, wenngleich auch einzelne Vorschläge zur weiteren Optimierung, wie z. B. Ergänzungen um zusätzliche Features (Einladung zum Bewerbungsgespräch, Zu- und Absage, Übernahme der Daten) formuliert werden. Insgesamt erleichtert die Praktikumsbörse die Abwicklung des Suchprozesses und vereinfacht das Angebot von Praktikumsstellen.

Die Jobbörse hat für unsere Praktika sehr gut funktioniert. Wir hatten ca. 2-3 Mal so viele Anfragen wie Stellen. Dadurch konnten wir die für unsere Aufgaben besten Kandidaten herausfinden und anstellen.

Alle notwendigen Informationen wurden uns über die Jobbörse von Anfang an zur Verfügung gestellt, was den Aufwand für den Praktikumsstart spürbar vereinfacht hat.

\subsection{2 | Charakteristika der Praktikantinnen und Praktikanten}

Um insbesondere das Interesse von Mädchen und jungen Frauen an FTI und naturwissenschaftlich-technischer Forschung zu steigern, wurden ab 2012 Quotenregelungen in die Ausschreibungen integriert. In den Jahren 2012 bis 2014 musste in allen Anträgen mit 3 oder mehr Praktika zumindest ein Drittel der Praktikumsplätze an Mädchen werden. Im Jahr 2012 traf dies nur auf Großunternehmen zu, ab 2013 auf alle teilnehmenden Organisationen. Ab 2015 wurde die Quotenregelung insofern geändert, als dass nun mindestens $50 \%$ der Praktika in einem Antrag an Schülerinnen und Schüler nicht-technischer Schulen vergeben werden müssen. 
Durch die Einführung der Quotenregelungen konnte der Anteil der weiblichen Praktikumsteilnehmerinnen kontinuierlich gesteigert werden. Im Jahr 2011 - ohne zahlenmäßige Vorgabe zur Einbindung von Mädchen - lag der Anteil der Praktikantinnen nur bei 32 \%. Durch die Regelung, ein Drittel der Praktikumsplätze für Mädchen bereitzustellen, wurde dieser Anteil nicht nur erreicht, sondern in den Jahren 2013 mit $38 \%$ und 2014 mit 36 \% auch überschritten. Aber vor allem durch die Vorgabe zum gezielten Angebot von Praktikumsplätzen für SchülerInnen nicht-technischer Schulen seit 2015 konnte der Anteil der Praktikantinnen deutlich gesteigert werden. Im Jahr 2019 lag der Anteil der Schülerinnen bereits bei $49 \%$.

Grafik 12 | PraktikumsteilnehmerInnen nach Geschlecht, Ausschreibungen 2011-2019, Anteile in Prozent

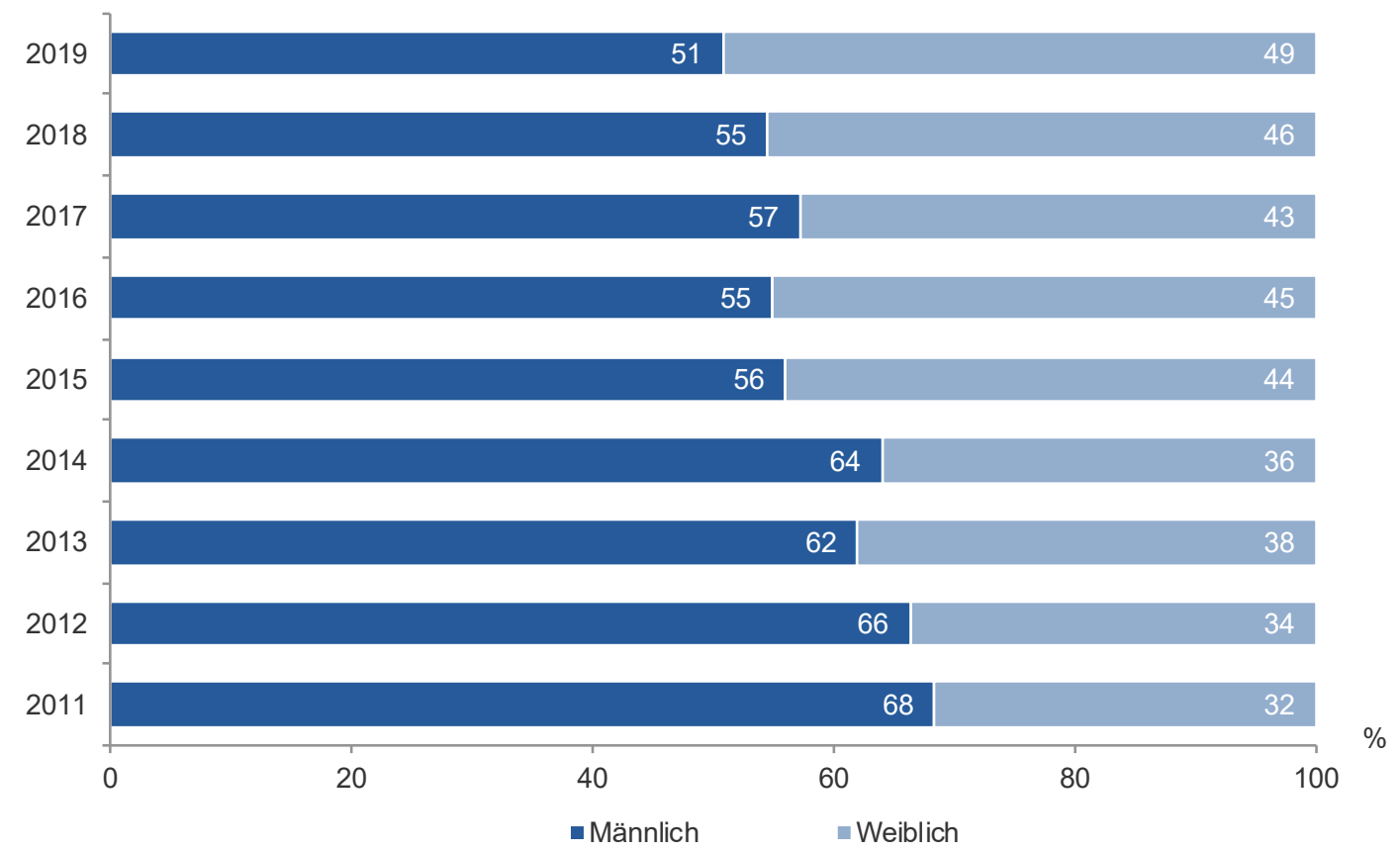

Quelle: FFG - Talente SchülerInnenpraktika Schlussauswertungen 2011-2019

Die schulische Herkunft der PraktikantInnen wird stark von der Quotenregelung beeinflusst. In den Jahren 2011 bis 2014 kamen nur jeweils knapp drei Zehntel der SchülerInnen aus einer allgemeinbildenden höheren Schule (AHS). Der Großteil rekrutierte sich aus berufsbildenden höheren Schulen (BHS) und hier vor allem aus höheren technischen Lehranstalten (HTL), da in diesen verpflichtenden Praktika vorgesehen sind.

Mit der Implementierung der Vorgabe zur Forcierung von Schülerlnnen aus nicht-technischen Schulen wurde der Anteil der PraktikantInnen aus einer AHS auf etwa $50 \%$ gesteigert. Im Jahr 2019 lag dieser bereits bei 53 \% (siehe Grafik 13). Da die nicht-technischen Schulen einen vergleichsweise höheren Anteil an Mädchen aufweisen, geht dies auch mit einer deutlichen Steigerung der Anzahl an weiblichen Praktikantinnen einher, wie auch Grafik 12 ab dem Jahr 2015 zeigt. 
Grafik 13 | Besuchte Schulen der PraktikantInnen, Ausschreibungen 2011-2019, in Prozent

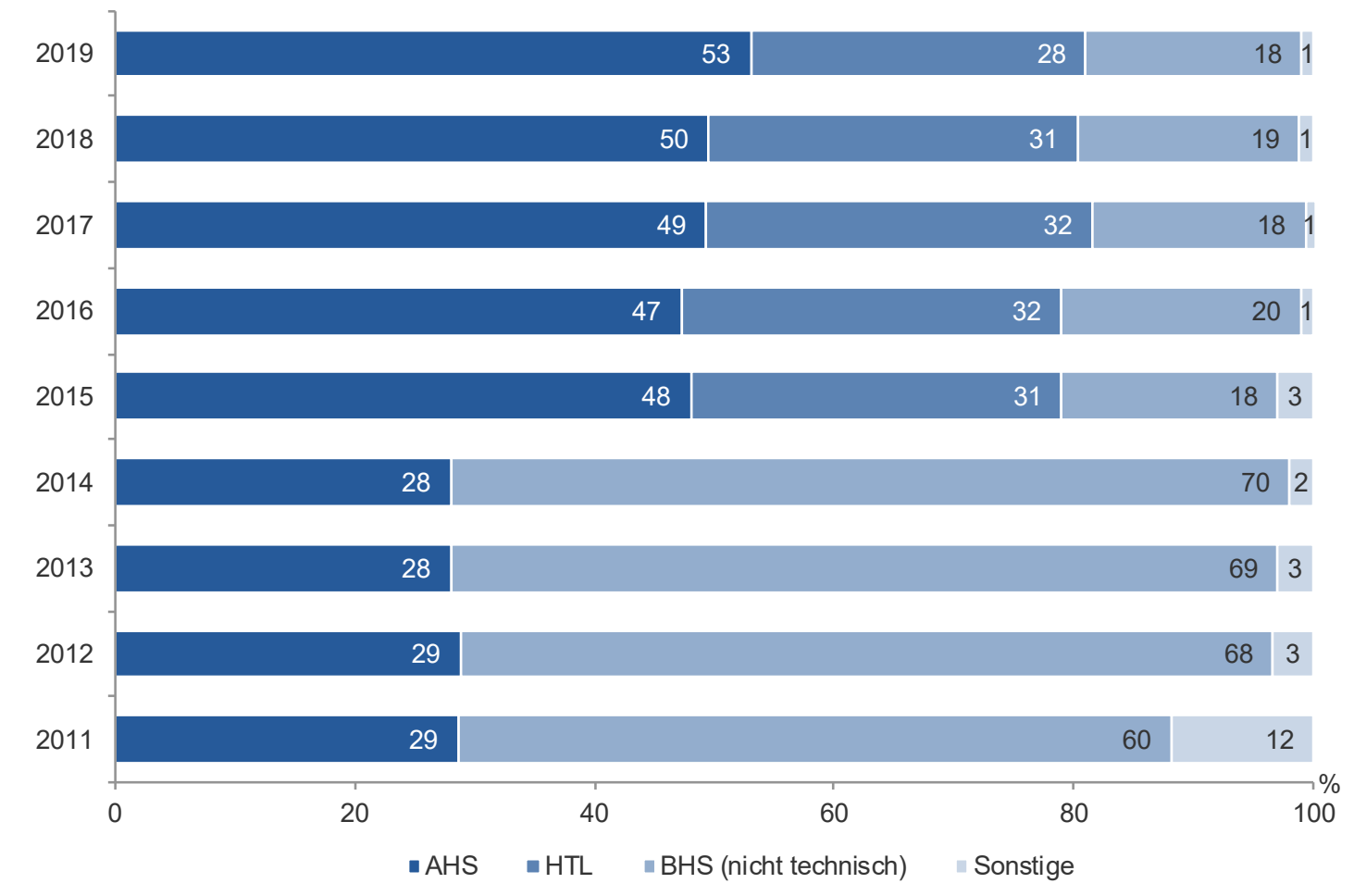

Anmerkung: Bis 2014 gab es keine Differenzierung von technischen und nicht-technischen BHS

Quelle: FFG - Talente SchülerInnenpraktika Schlussauswertungen 2011-2019

Die Quotenregelungen führten sowohl bei der Einführung der „Drittelregelung für Mädchen“ als bei der Reservierung der Hälfte der Praktikumsplätze für SchülerInnen nicht-technischer Schulen zu einer gewissen Kritik von Seiten der Fördernehmerlnnen. Als problematisch stellte sich für diese vor allem heraus, dass nicht ausreichend Mädchen bzw. SchülerInnen aus allgemeinbildenden Schulen gefunden werden konnten, und sich daher der Aufwand im Zuge des Auswahlprozesses erhöhte.

Wir hätten uns mehr Bewerber von nicht-technischen Schulen gewünscht.

Es gibt zu wenig Bewerbungen von AHS Schülern. Da für jeden HTL Schüler ein AHS Schüler aufgenommen werden muss, ist die Anzahl der durchführbaren Praktika durch die Anzahl der verfügbaren AHS Schüler limitiert.

Um Vorurteilen der FördernehmerInnen gegenüber AHS-SchülerInnen entgegenzuwirken, wäre es laut ExpertInnenmeinung wichtig, auf die Vorteile der AHS hinzuweisen. Allgemeinbildende Schulen verfügen teilweise auch über technische Schwerpunkte und schulen logisches Denken, beispielsweise im Rahmen des Lateinunterrichts.

Generell herrscht aber großes Verständnis für diese Vorgaben, um das Interesse von Mädchen und nicht technik-affinen Personen für naturwissenschaftliche und technische Themen zu wecken. Die Quotenregelung bewirkte, dass die Unternehmen und Forschungseinrichtungen auch 
Personen ohne technischen Schulhintergrund als PraktikantInnen aufnehmen, die sonst nicht in ihrer Personalplanung vorgesehen gewesen wären. Die Einbindung dieser Personen war in der Praxis manchmal mit mehr Aufwand verbunden, brachte aber auch neue Erkenntnisse und Sichtweisen für die Fördernehmerlnnen.

Die Quotenregelung ist gelungen, da auch Jugendlichen ohne technischen Hintergrund die Möglichkeit gegeben wird in Berufsfelder einzublicken, in denen die Praktikumsstellen sonst wahrscheinlich an Schülerlnnen aus technischen Schulen bevorzugt vergeben werden würden.

Die Kombination aus nicht-technischen Praktikanten und technischen Praktikanten hat den Praktikanten und unserem Unternehmen neue Sichtweisen gebracht.

Der Vergleich der PraktikantInnen nach Schultypen macht aber dennoch deutlich, dass AHSSchülerInnen vorwiegend in Forschungseinrichtungen ein Praktikum absolvieren, und hier insbesondere in Universitäten. Nur ein Drittel der AHS-SchülerInnen absolviert ihr/sein Praktikum in einem Unternehmen. Demgegenüber bekommen Jugendliche, die eine HTL oder eine (nichttechnische) BHS besuchen, wesentlich häufiger die Gelegenheit, in einem Unternehmen ein gefördertes Praktikum zu absolvieren (54 \% bzw. 66 \%). Die Universitäten bieten den AHS-SchülerInnen zwar einen sehr hochwertigen Einblick in die wissenschaftliche Forschung, der konkrete Bezug zur Wirtschaft und unternehmerischen Praxis fehlt hier allerdings.

Grafik 14 | Anteile der PraktikantInnen aus den verschiedenen Schultypen nach Organisationsarten, Ausschreibungen 2015-2019, in Prozent

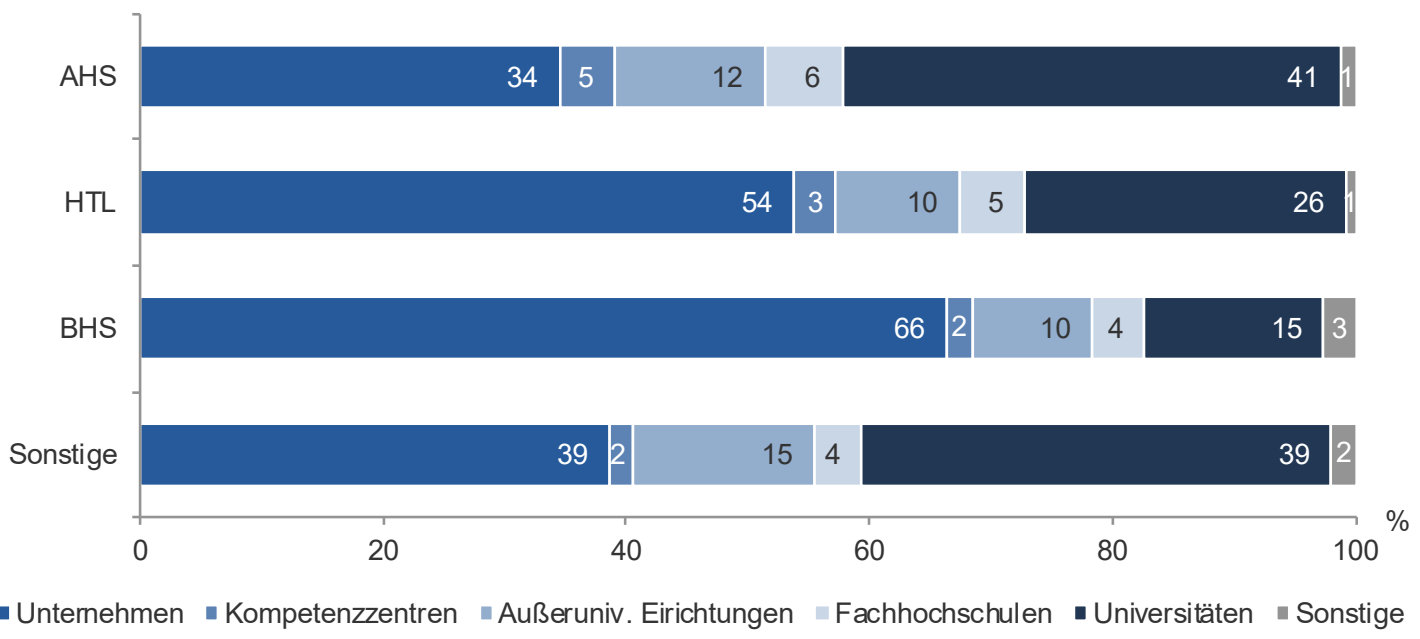

Quelle: FFG; Berechnung und Darstellung KMU Forschung Austria 


\subsubsection{Beurteilung und Wirkungen der Praktika für Schülerinnen und Schüler}

Die Förderung im Rahmen von Talente Praktika für Schülerinnen und Schüler ermöglicht vielen Einrichtungen erst, sich der Schulung von jungen Nachwuchskräften zu widmen. Ohne finanzielle Unterstützung wäre dies oftmals nicht möglich. Durch das Angebot von Praktikumsplätzen ist auch das Interesse der teilnehmenden Organisationen an der aktiven Nachwuchsförderung großteils (sehr) gestiegen. In den Jahren 2011 bis 2019 berichten zwischen $73 \%$ und $81 \%$ der FördernehmerInnen von einem gestiegenen Interesse, 2019 traf dies auf $76 \%$ zu. Es bietet den teilnehmenden Unternehmen und Forschungseinrichtungen vor allem die Möglichkeit, schon SchülerInnen naturwissenschaftliche oder technische Themen näherzubringen.

Ohne die Ausschreibung wären die Praktika an unserer Organisation nicht durchgeführt worden. Die Ausschreibung hat uns die wertvolle Erfahrung ermöglicht, mit Jugendlichen zu arbeiten (ansonsten nur ältere Studierende).

Wir könnten ohne diese Förderung leider keine F\&E Praktika anbieten. Aber es macht uns sehr viel Freude, jungen Menschen die Chance zu geben, sich in diesem Bereich weiterzuentwickeln und zukünftige Talente zu begleiten und zu fördern.

Etwa die Hälfte der teilnehmenden Organisationen schreibt den Praktika einen unmittelbaren Nutzen zu (siehe Grafik 15). Dieser besteht in erster Linie darin, SchülerInnen möglichst früh einen Einblick in den beruflichen Alltag zu geben und innen naturwissenschaftliche und technische Themen näher zu bringen.

Die Ausschreibung ermöglicht naturwissenschaftlich-interessierten jungen Menschen, Forschung und Entwicklung hautnah mitzuerleben und einen realen Einblick in dieses Berufsfeld zu gewinnen.

Das ist eine gute Möglichkeit SchülerInnen technische Themen näher zu bringen, die im Alltag nicht so präsent sind.

Mehr als $60 \%$ sehen in der Einbindung von SchülerInnen in die F\&E-Aktivitäten des Unternehmens bzw. der Forschungseinrichtung im Zeitraum von 2011 bis 2019 einen langfristigen Nutzen. Dieser bezieht sich darauf, das Interesse von jungen Menschen für F\&E-Aktivitäten sowie naturwissenschaftliche und technische Themen zu wecken und damit ihre weitere Studien- und Berufswahl entsprechend zu beeinflussen. Ein weiterer langfristiger Vorteil ist, dass frühzeitig Kontakte zwischen Unternehmen und Jugendlichen hergestellt werden, welche bei der Suche nach potentiellen Arbeitskräften hilfreich sein können.

Das Interesse der SchülerInnen an F\&E Aktivitäten ist geweckt worden, sie haben aufgrund ihres Praktikums nützliche Anregungen für ihre vorwissenschaftlichen Arbeiten bzw. für ihre zukünftige Studienauswahl mitgenommen.

Das Programm bietet dafür eine sehr gute Plattform und eine Chance uns als attraktiven Arbeitgeber präsentieren zu können um Jugendliche für unser Berufsfeld zu begeistern und ev. auch als spätere Mitarbeiter zu gewinnen. 
Das Förderprogramm hilft uns mit potentiellen zukünftigen Mitarbeitern Kontakt aufzunehmen und diese besser kennenzulernen. Über das Programm wurden bereits aus mehreren ehemaligen Praktikanten neue Mitarbeiter.

Nur rd. $5 \%$ empfinden, dass das Angebot von geförderten Praktika mit mehr Aufwand als Nutzen verbunden ist.

Grafik 15 | Beurteilung des Nutzens der Praktika für SchülerInnen für die FördernehmerInnen, Ausschreibungen 2011-2019, in Prozent (Mehrfachnennungen möglich)

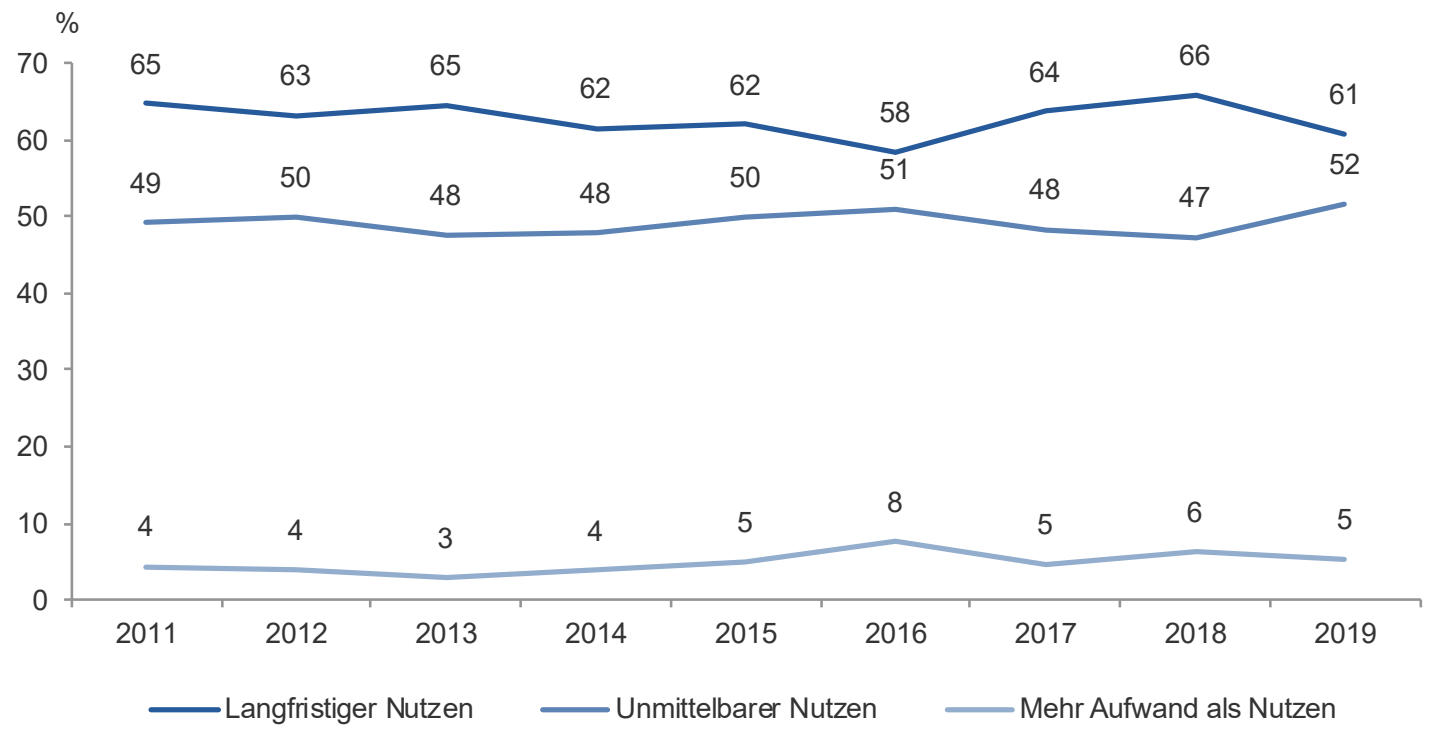

Quelle: FFG - Talente SchülerInnenpraktika Schlussauswertungen 2011-2019

Insgesamt ist die Zufriedenheit der teilnehmenden Unternehmen und Forschungseinrichtungen so groß, dass die Mehrheit auch in Zukunft Praktika für Schülerinnen und Schüler anbieten möchte. Zwischen $63 \%$ und 73 \% geben an, dass sie auch in Zukunft sicherlich Praktikumsplätze zur Verfügung stellen wollen, 2019 trifft es auf $68 \%$ zu. Darüber hinaus halten zwischen $27 \%$ und $33 \%$ das weitere Angebot von SchülerInnenpraktika für wahrscheinlich, 2019 sind es $28 \%$.

Auch die SchülerInnen, die im Zeitraum von 2011 bis 2019 geförderte Praktika absolviert haben, sind mit diesen sehr zufrieden. Zwischen $64 \%$ und $84 \%$ geben an, dass innen ihr Praktikum sehr gut gefallen hat. 2019 waren es fast zwei Drittel (65\%). Im Rahmen der Praktika konnten sich die Jugendlichen neues Wissen aneignen und erste Erfahrungen in der Arbeitswelt sammeln. Die Praktikumsoption hat innen einen Einblick in neue Berufsfelder und Forschungsbereiche gewährt, von denen sie teilweise gar nicht wussten, dass diese existieren. Vor allem SchülerInnen aus einer AHS waren von dieser Möglichkeit begeistert.

Das Praktikum hat sich gelohnt, weil...

...ich viele tolle Eindrücke in der Forschung bekommen habe, die ich ansonsten wahrscheinlich nie in meinem Leben bekommen hätte. 
... ich in einen Bereich (Forschung) hineinschnuppern konnte, den ich im Laufe meiner Schulzeit an einer AHS nicht kennenlernen kann.

...finde, dass so ein Praktikum sehr gut ist (besonders für AHSler), da man als AHSler selten ein Praktikum bekommt und hier wird man bevorzugt und kann somit auch einen Einblick ins technische Arbeitsumfeld bekommen.

Grafik 16 | Beurteilung der Praktika durch die SchülerInnen, Ausschreibungen 2011-2019, in Prozent

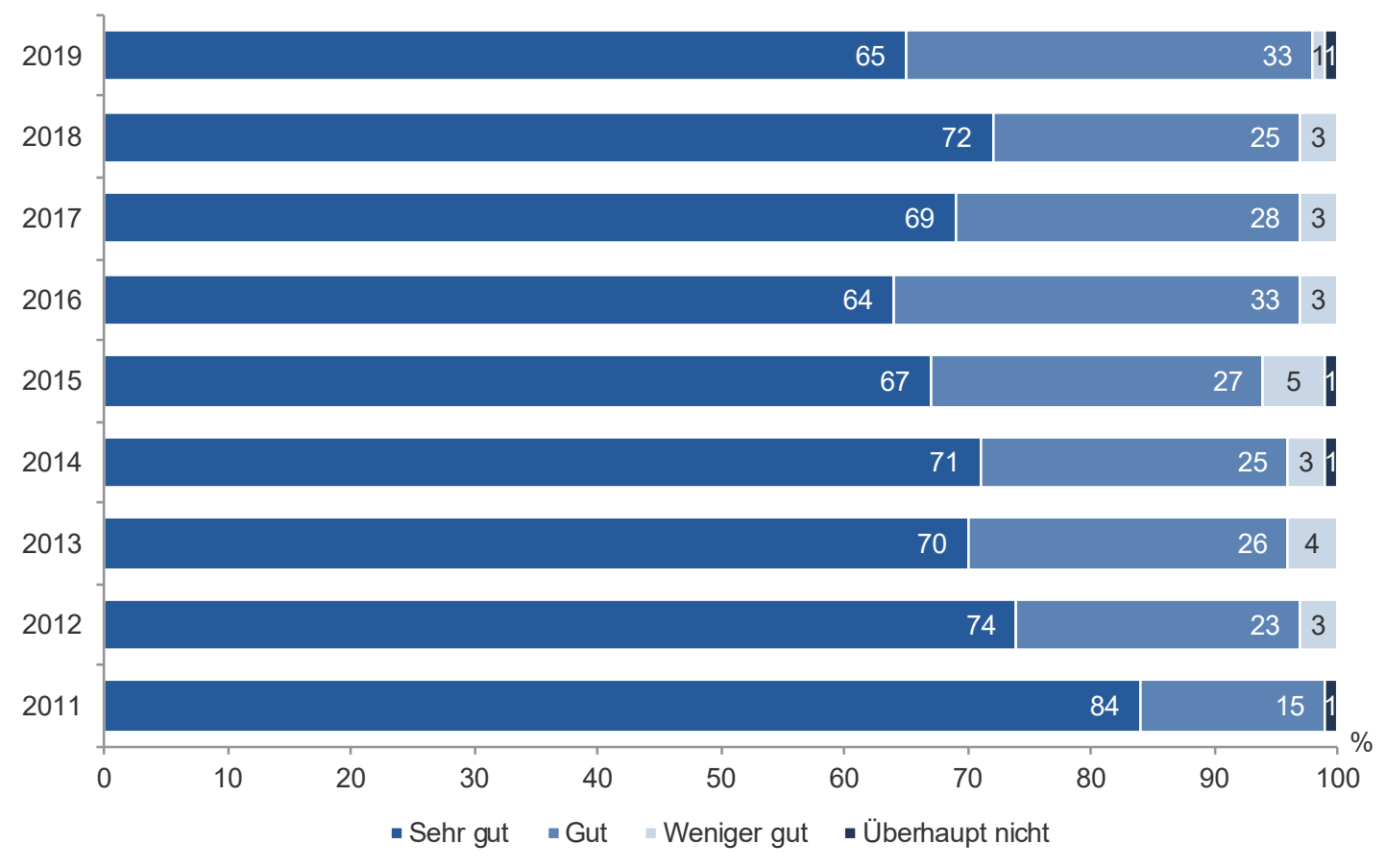

Quelle: FFG - Talente Schülerlnnenpraktika Schlussauswertungen 2011-2019

Die SchülerInnen haben im Rahmen ihrer Praktika großteils (von $92 \%$ bis $78 \%$ ) anspruchsvolle Tätigkeiten ausgeübt und viel dazugelernt. Daher ist bei $73 \%$ bis $57 \%$ auch das Interesse an naturwissenschaftlich-technischen Berufen gestiegen. 2019 geben $66 \%$ der Schülerlnnen an, dass naturwissenschaftliche-technische Themen stärker in ihren Interessensfokus gerückt sind. (siehe Grafik 17) Die Praktika haben sich somit für viele Jugendliche als wichtige Orientierungshilfe für den weiteren Bildungs- und Berufsweg erwiesen. 
Das Praktikum hat sich gelohnt, weil...

...ich extrem spannende Einblicke in Forschung und Wissenschaft bekommen habe. Meine Betreuer haben immer versucht mir alles zu erklären und sich extrem viel Zeit für mich genommen. Ich habe extrem viel gelernt!

...es eine sehr spannende Erfahrung war und ich nun eine sehr viel bessere Vorstellung von dem habe, was ich einmal machen möchte.

...ich nun einen Einblick in die Arbeitswelt bekommen habe und mir ziemlich sicher bin, dass ich einmal in einem technisch / naturwissenschaftlichen Bereich arbeiten will.

Nur einige wenige Schülerlnnen geben an, dass das Praktikum nicht ihren Wünschen oder Vorstellungen entsprochen hat, weil sie beispielsweise unterfordert waren, Leerlaufzeiten oder die BetreuerInnen zu wenig Zeit für sie hatten.

Grafik 17 | Bewertung der Tätigkeiten und Einschätzung des Interesses an naturwissenschaftlichen Berufen durch die SchülerInnen, Ausschreibungen 2011-2019, in Prozent

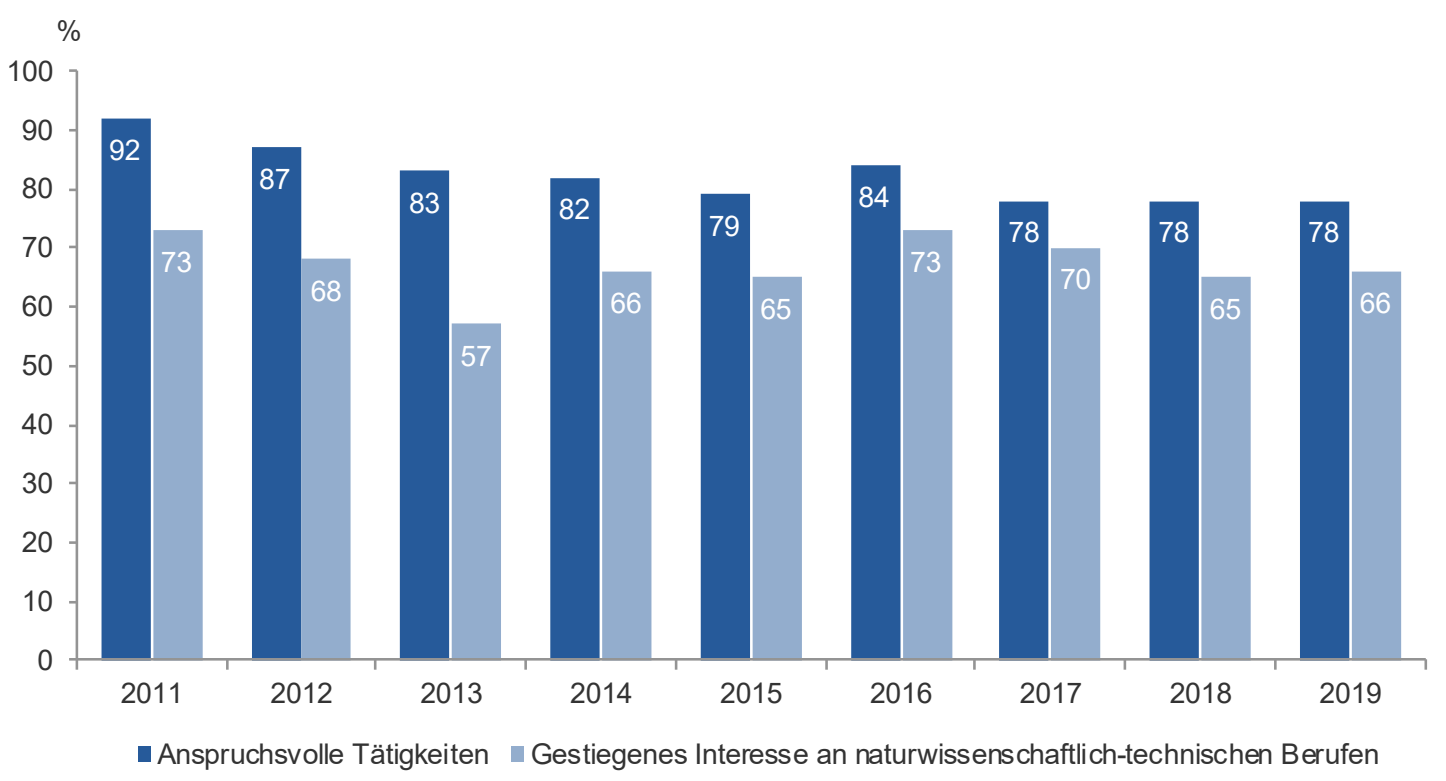

Quelle: FFG - Talente SchülerInnenpraktika Schlussauswertungen 2011-2019

Sowohl die Schülerlnnen als auch die teilnehmenden Organisationen sind mit der Abwicklung und Umsetzung der geförderten Praktika für Schülerinnen und Schüler äußerst zufrieden. Dennoch werden vereinzelt Verbesserungsvorschläge zur weiteren Optimierung des Praktikumsangebots erwähnt. Diese betreffen die Erweiterung der Dauer der Praktika (auf bis zu 2 Monate), die Erhöhung der Förderung, die Ausdehnung des Praktikumsangebots auf forschungsnahe Bereiche (wie z. B. Design, Architektur, Medizin) oder die Erweiterung der Zielgruppen auf z. B. ältere Jugendliche oder StudentInnen. Abgesehen von diesen inhaltlichen Adaptierungsvorschlägen wird vor allem angeregt, die Praktika für Schülerinnen und Schüler sowohl an Schulen als auch bei den Unternehmen stärker zu bewerben. Vor allem im Bereich der AHS werden noch Informationsdefizite bezüglich der Option eines geförderten Praktikums geortet. 
Es wissen grundsätzlich viel zu wenige Firmen und Schulen, dass es dieses Programm gibt! Promotion dafür könnte verbessert werden.

An den AHS sollten die Praktika besser bekannt gemacht werden. In den HTLs sind die Schüler mittlerweile gut informiert, die AHS Schüler haben oft keine Informationen.

Die öffentliche Sichtbarkeit des Förderschwerpunktes "Talente" könnte verbessert werden, sodass nicht nur über die FFG-Homepage, sondern auch über weitere Printmedien oder soziale Medien auf die Möglichkeit aufmerksam gemacht wird.

Die Praktika für Schülerinnen und Schüler werden von den befragten Expertlnnen als spannender Einstieg in naturwissenschaftliche und technische Karrieren gesehen. Es wird jedoch darauf hingewiesen, dass diese Möglichkeit tendenziell eher von interessierten und engagierten Schülerlnnen mit einem unterstützenden sozialen Umfeld in Anspruch genommen wird. Wenn dieses fehlt, müssten vor allem Lehrkräfte und Schulen verstärkt auf diese Option hinweisen. Dazu muss aber verstärkte Informationsarbeit im schulischen Bereich, insbesondere bei den AHS, geleistet werden. In diesem Zusammenhang sind auch kreative Ideen gefragt, um verstärkt AHS-SchülerInnen und Unternehmen zusammenzubringen, da bei dieser Schulform keinerlei berufliche Praxis vorgesehen ist. Dazu könnten beispielsweise ein zusätzliches Förderinstrument oder eine Art „Dating-Portal für SchülerInnen und Unternehmen“ geschaffen werden. Die Förderung der Praktika für Schülerinnen und Schüler sollte aus ExpertInnensicht ein möglichst breites Publikum an SchülerInnen miteinbeziehen und nicht zur Förderung von Pflichtpraktika dienen. 


\section{2 | Talente regional}

Talente regional verfolgt das Ziel, mehr junge Menschen für die Forschung zu begeistern. Dazu werden regionale Projekte gefördert, bei denen mindestens 2 UnternehmenspartnerInnen, ein/e wissenschaftliche/r PartnerIn und 5 Bildungseinrichtungen vom Kindergarten bis zu höheren Schulen miteinander kooperieren und altersgerechte, praxisnahe Aktivitäten in den Bereichen Naturwissenschaft und Technik initiieren. Dabei setzen sich die Kinder und Jugendlichen mit spannenden Themen auseinander, experimentieren, forschen und lernen Tätigkeiten und Berufsbilder in Forschung, Technologie und Innovation kennen. Um weitere Bildungseinrichtungen einzubeziehen und das regionale Netzwerk zu vergrößern, werden pro Projekt zehn Kooperationszuschüsse in der Höhe von pauschal $€ 1.000$ für weitere Schulen und Kindergärten vergeben.

Tabelle 2 | Eckpunkte zu Talente regional

\begin{tabular}{|c|c|}
\hline Talente regional & Kurzbeschreibung \\
\hline Ziele & $\begin{array}{l}\text { Das Interesse von Kindern und Jugendlichen unabhängig ihres Geschlechts, ihrer } \\
\text { sozialen oder geographischen Herkunft an FTI steigern, ihren Bezug zu Naturwissen- } \\
\text { schaft und Technik vertiefen und sie für eine Karriere in der angewandten Forschung } \\
\text { gewinnen. } \\
\text { Bildungseinrichtungen, Wirtschaft und Forschung bei innovativen Themen vernetzen. }\end{array}$ \\
\hline Zielgruppen & $\begin{array}{l}\text { Unternehmen, Einrichtungen für Forschung und Wissensverbreitung, Universitäten, } \\
\text { Fachhochschulen, Pädagogische Hochschulen, außeruniversitäre Forschungsein- } \\
\text { richtungen, Technologietransfer-Einrichtungen, InnovationsmittlerInnen etc. (Förder- } \\
\text { nehmerInnen) } \\
\text { Kinder und Jugendliche österreichischer, (vor-)schulischer Bildungseinrichtungen } \\
\text { Ab } 2014 \text { besonderer Fokus auf Kinder, Jugendliche, Eltern und Forscherlnnen mit } \\
\text { Migrationshintergrund }\end{array}$ \\
\hline Inhalt & $\begin{array}{l}\text { PartnerInnen aus Wirtschaft und Forschung entwickeln gemeinsam mit Bildungsein- } \\
\text { richtungen Projekte für Kinder und Jugendliche, die es ermöglichen, sich mit den The- } \\
\text { men Forschung, Technologie und Innovation in den Bereichen Naturwissenschaft } \\
\text { und Technik auseinander zu setzen. Die Projekte werden in räumlicher Nähe unter } \\
\text { Berücksichtigung von Gender-Aspekten umgesetzt und dienen dazu, die Vernetzung } \\
\text { sowie innovative pädagogische Konzepte nachhaltig zu fördern. }\end{array}$ \\
\hline Konsortium & $\begin{array}{l}\text { Die Kooperation mehrerer Partnerlnnen im Rahmen eines Konsortiums in Form von: } \\
\text { - } \quad \text { mind. } 1 \text { wissenschaftliche/r Partnerln } \\
\text { - } \quad 5 \text { Bind. } 2 \text { Unternehmen } \\
\text { kundarstufe II) } \\
\quad 2011-2014 \text { : Einbindung von mindestens } 3 \text { der } 4 \text { Bildungsstufen } \\
\quad \text { ab } 2015 \text { Einbindung von mind. } 2 \text { Volksschulen und mind. } 2 \text { Sekundarstufen I }\end{array}$ \\
\hline $\begin{array}{l}\text { Einreichverfah- } \\
\text { ren }\end{array}$ & $\begin{array}{l}\text { Ausschreibungen in den Jahren 2011, 2012, 2013, 2014, 2015, 2016, } 2019 \\
\text { Einreichfristen: 2011: Oktober bis Februar } \\
\qquad \text { ab } 2012 \text { jeweils September - Dezember }\end{array}$ \\
\hline Laufzeit & $\begin{array}{l}2011 \text { - 2014: Projektdauer } 12 \text { bis } 18 \text { Monate } \\
\text { 2015, 2016, 2019: Projektdauer } 24 \text { bis } 36 \text { Monate }\end{array}$ \\
\hline Förderhöhe & $\begin{array}{l}2011-2014: \text { bis zu } € 60.000,- \text { (inkl. } € 10.000 .- \text { Kooperationszuschüsse) } \\
2015,2016,2019: \text { bis zu } € 130.000 \text { (inkl. } € 10.000 \text { Kooperationszuschüsse) }\end{array}$ \\
\hline Spezifika & $\begin{array}{l}\text { 2013: Schwerpunkt auf den Themen Mobilität und Energie } \\
\text { Ab 2014: Ausschreibungsschwerpunkt Migrationshintergrund (von Kindern und Ju- } \\
\text { gendlichen, Forscherlnnen als Role Models, interkulturelle Kompetenzen) }\end{array}$ \\
\hline
\end{tabular}




\subsubsection{Struktur der geförderten Talente regional Projekte}

Im Zeitraum von 2011 bis 2019 wurden insgesamt 141 Talente regional Projekte gefördert. Die meisten Projekte, nämlich 24, konnten im Rahmen der Ausschreibung im Jahr 2013 realisiert werden. In diesem Jahr profitierten jedoch 8 Projekte von der finanziellen Unterstützung durch den Klima- und Energiefonds (KLIEN). Bei der Ausschreibung 2019 konnten trotz 2-jähriger Ausschreibungspause nur 22 Projekte eine Förderung lukrieren. Die steigende Anzahl an Einreichungen im Zeitverlauf weist auf das steigende Interesse von Wissenschaft, Wirtschaft und Bildungseinrichtungen hin, sich aktiv der Nachwuchsförderung zu widmen. Gleichzeitig kann der steigende Wettbewerb bei der Auswahl der Förderungen laut ExpertInnenmeinung auch etwas abschreckend wirken, da die Antragstellung mit viel Arbeit verbunden ist. Da nunmehr die Ausschreibungen nur alle zwei Jahre stattfinden sollen (im Wechsel mit FEMtech Forschungsprojekte), wird auch weiterhin eine hohe Anzahl an Anträgen zu erwarten sein.

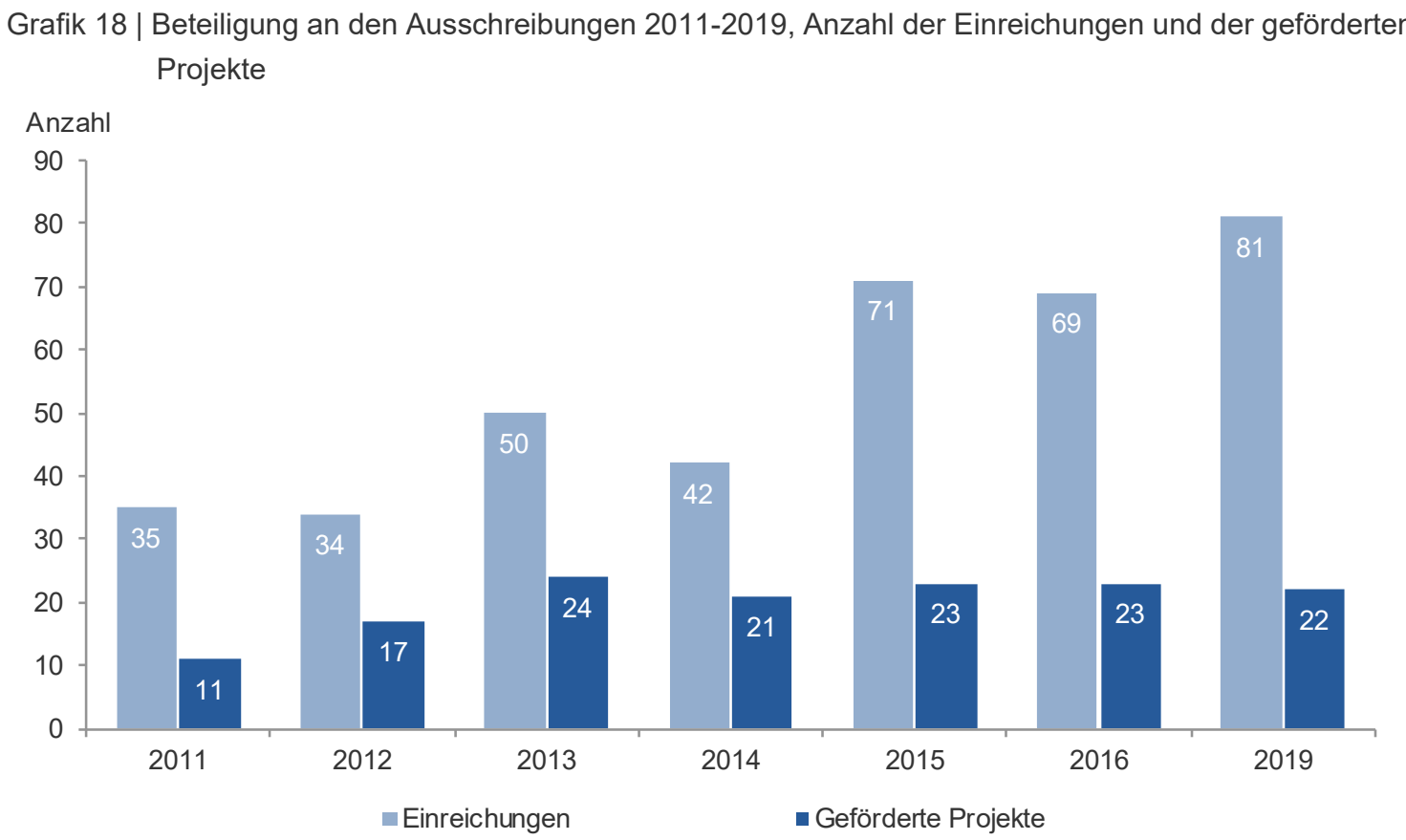

Quelle: FFG; Berechnung und Darstellung KMU Forschung Austria

Inhaltlich konzentrieren sich die Talente regional Projekte im Zeitraum von 2011 bis 2019 auf die Themenbereiche Energie/Umwelt, Life Sciences und Mobilität, wie Grafik 19 zeigt. Entsprechend des Schwerpunkts auf Mobilität und Energie im Jahr 2013 konzentrierte sich jeweils ein Viertel der Projekte darauf, aber auch im Jahr 2015 wurden 30 \% der Projekte im Bereich Mobilität realisiert. Energie- und Umweltthemen standen bei den Ausschreibungen in den Jahren 2011, 2012 und 2019 im Vordergrund, obwohl es diesbezüglich keine Schwerpunktsetzung gab. Life Sciences spielten vor allem im Jahr 2011, aber auch 2013 und 2015 eine große Rolle. 2016 standen hingegen vor allem Projekte in der Produktion im Vordergrund. 
Grafik 19 | Themenfelder der Projekte nach Ausschreibung 2011-2019, in Prozent

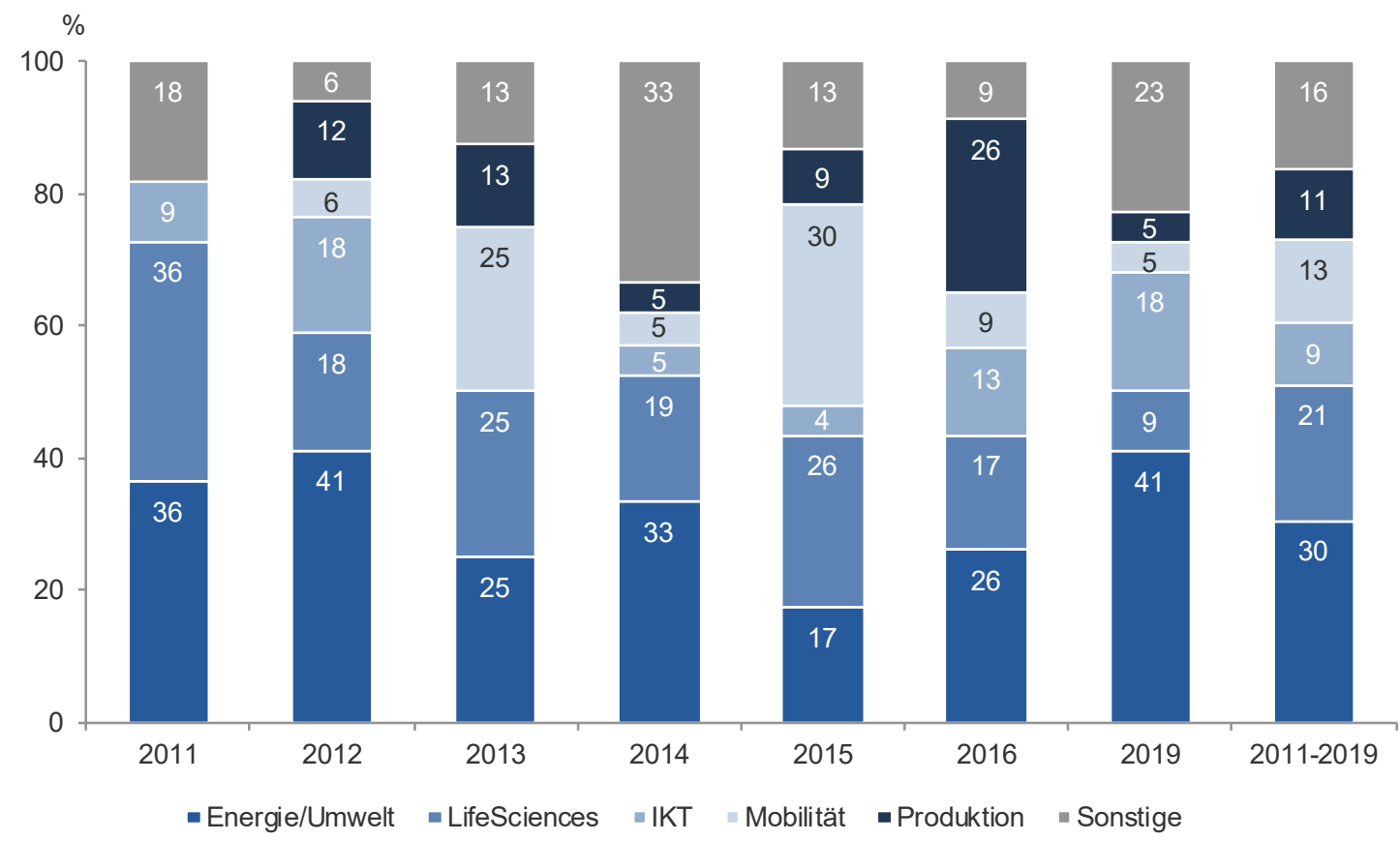

Quelle: FFG; Berechnung und Darstellung KMU Forschung Austria

Konkret werden im Rahmen der Energie- und Umweltthemen Projekte zur Steigerung der Energieeffizienz umgesetzt, praktische Möglichkeiten zu Energieeinsparungen im Bereich der Wärme oder Beleuchtung erforscht, der Energiehaushalt im eigenen Umfeld bzw. speziell in Migrantlnnenfamilien analysiert, Ideen für effiziente Energiespeicherung gewonnen oder das Thema Wasserkraft erforscht. Als Umweltthemen wurde Bodenwissen erworben, sich dem Bodenschutz oder dem Wasserfluss gewidmet sowie Schnee, Eis, Luft, Licht und Pflanzen näher analysiert. Darüber hinaus wurden Klimaveränderungen betrachtet, Möglichkeiten der energieeffizienten Gemüseund Lebensmittelproduktion erforscht, die nachhaltige Rohstoffgewinnung, Recycling und Stoffkreisläufe analysiert, Technik-Know-how, insbesondere von Mädchen, aufgebaut und den Kindern und Jugendlichen Umweltberufe nähergebracht.

Im Bereich der Life Sciences erfolgt beispielsweise die Analyse von Lebensmitteln und Allergenen, der Rolle von Enzymen sowie von Biomarkern, z. B. als Hinweise für Herzerkrankungen. Auch verschiedene Forschungsmethoden im Bereich der Optik und Akustik, der Gentechnik oder Biotechnologie werden erprobt und den Kindern und Jugendlichen im Rahmen von Experimenten nähergebracht. Der Körper, Krankheiten und Gesundheitsthemen werden mit Hilfe naturwissenschaftlicher Technik erlebbar gemacht, Wege von Forschungsideen zu Produktinnovationen (z. B. im Bereich implantierbarer Hörlösungen) aufgezeigt und die Arbeit in Forschungslaboren durch praktische Mitarbeit demonstriert. 
Die Projekte im IKT-Bereich konzentrieren sich vor allem auf die Robotik-Forschung mit verschiedenen Robotik- und Prototypen-Workshops. Auch die Informatik wurde den Kindern und Jugendlichen durch experimentelles Erarbeiten informationstechnologischer Grundlagen nähergebracht, der 3D-Druck erprobt und interaktive Internet-Kartenapplikationen für und von der Zielgruppe beispielhaft für die Stadt Salzburg entwickelt.

Die Mobilitätsprojekte fokussieren auf die Optimierung von Verkehrswegen und Verkehrsmitteln, wie z. B. Fahrrädern, um die aktive, selbstbestimmte und sichere Mobilität von SchülerInnen zu unterstützen oder die Lebensqualität in Straßennähe zu verbessern. Schul- und Verkehrswege werden erforscht, um anhand konkreter Beispiele Themen wie Green Mobility, E-Mobilität, Mobilitätsforschung, Verkehrsplanung und -sicherheit den Kindern und Jugendlichen näher zu bringen. Auch neue pädagogische Ansätze, wie der Einsatz einer Handpuppe oder des spielbasierten Lernens, werden in der Mobilitätsforschung erprobt, die Geheimnisse des Fliegens durch praktische Einblicke in Luft- und Raumfahrt erforscht und Teilbereiche einer Smart-City in Bezug auf Energiegewinnung, Verkehr, Solar-Fahrzeuge, Raum für Familie etc. konzipiert.

Im Bereich der Produktion werden praktische Einblicke in neue Technologien im Produktionsprozess gewährt und z. B. im Rahmen eines 3D Schnupperlabors eigene Produkte konstruiert oder Fahrzeugbau und Anlagentechnik experimentell vermittelt. Auch verschiedene Werkstoffe, wie Holz, Metall und Kunststoff und deren Wertschöpfungskreisläufe werden erforscht sowie innovative, zukunftsträchtige Anwendungsmöglichkeiten und anschauliche Praxisbeispiele aufgezeigt. Gleichzeitig werden neue Berufsfelder in diesen Bereichen vorgestellt.

Bei der Umsetzung der Talente regional Projekte wird interdisziplinär zusammengearbeitet und der Austausch zwischen Wirtschaft, Wissenschaft und Bildungseinrichtungen intensiviert. Die PädagogInnen werden maßgeblich in die Projektaktivitäten eingebunden, um das forschende Lernen an den Schulen zu forcieren. Dazu werden Workshops mit den PädagogInnen beispielsweise zu den Themen Experimentieren, Umgang mit Technik, geschlechter- und sprachsensible Methodik / Didaktik veranstaltet, um sie zu schulen, wie sie die Forschungsinhalte im Unterricht vermitteln können. Darüber hinaus werden die Talente regional Projekte auch dazu genutzt, neue pädagogische Konzepte und Workshops zu erproben, bevor sie in den regulären Betrieb übernommen werden.

Hauptzielgruppe der Talente regional Projekte sind Kinder und Jugendliche unterschiedlicher Altersgruppen, die aktiv in das jeweilige Forschungsthema eingebunden werden. Dabei werden besonders Mädchen und seit 2014 auch gezielt Kinder und Jugendliche mit Migrationshintergrund angesprochen. Für die Nachwuchsforscherlnnen werden häufig Workshops veranstaltet, um innen praxisorientiert und durch aktive Mitarbeit den jeweiligen Forschungsgegenstand näherzubringen. Dazu werden praktische Experimente, Forschungstage und Exkursionen in die Natur sowie zu Unternehmen, Universitäten und Forschungseinrichtungen durchgeführt, um Umweltund Energiefragen zu erforschen, Werkstoffe und Mobilitätskonzepte zu erkunden, Körper- und Gesundheitsfragen zu lösen oder neue, innovative IT-Lösungen kennenzulernen. Auf die aktive Einbindung der Kinder und Jugendlichen wird großen Wert gelegt, wobei sie teilweise selbst in die Rolle von Forscherlnnen oder Lehrenden schlüpfen oder Produkte zum jeweiligen For- 
schungsprojekt herstellen. Über die Auseinandersetzung mit den verschiedenen Forschungsinhalten werden den Kindern und Jugendlichen auch die Berufsfelder und Karrieremöglichkeiten im Bereich von Forschung, Naturwissenschaft und Technik nähergebracht.

Um die Projektergebnisse einer breiteren Öffentlichkeit, wie anderen Bildungseinrichtungen, regionalen AkteurInnen, den Eltern etc., vorzustellen, werden Tagungen, Workshops, Präsentationen, Ausstellungen und Forschungsfeste veranstaltet. Auch die Projekt-Websites unterstützen bei der Verbreitung der neu entwickelten Unterrichtsmaterialien und Workshop-Konzepte. Videos, Filme und Medienbeiträge sowie die Teilnahme an Wettbewerben und Forschungsveranstaltungen tragen zur weiteren Informationsverbreitung bei.

\subsection{2 | Beschreibung der Projektteilnehmerlnnen}

Von den FördernehmerInnen bilden die Unternehmen die größte Gruppe, wobei sich zwischen 2011 und 2019 vor allem Kleinunternehmen im Bereich der Nachwuchsförderung engagieren und sich an Talente regional Projekten beteiligen. Weitere bedeutende AkteurInnen stellen die Hochschulen dar, gefolgt von den außeruniversitären Forschungseinrichtungen.

Grafik 20 | Verteilung der Fördernehmerlnnen, Ausschreibungen 2011-2019, in Prozent

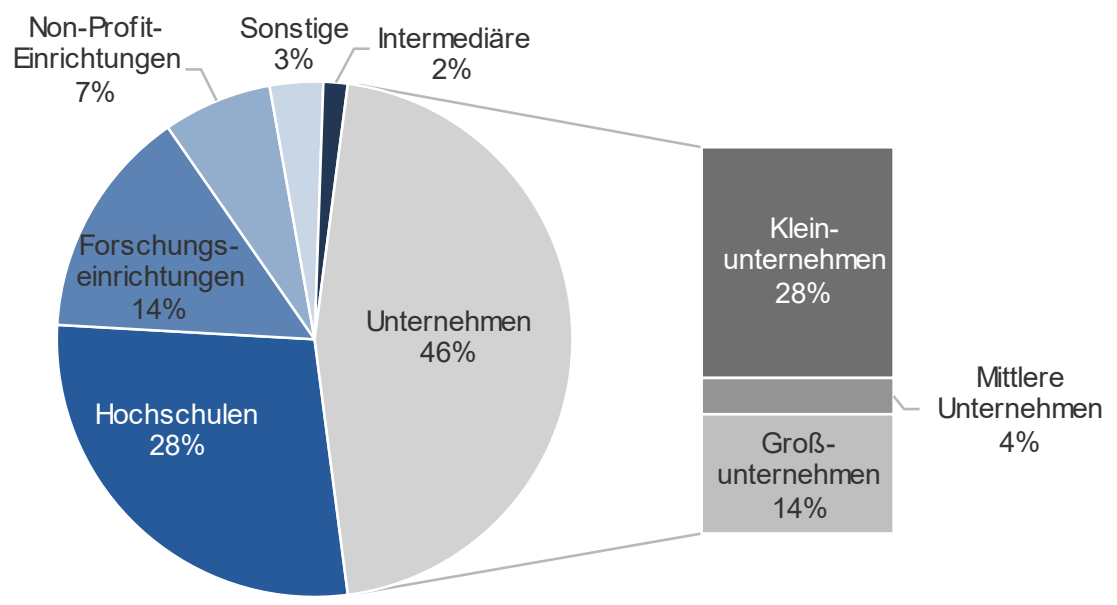

Quelle: FFG; Berechnung und Darstellung KMU Forschung Austria

In Hinblick auf die Konsortialführung der 141 zwischen 2011 und 2019 realisierten Talente regional Projekte zeigt sich, dass Unternehmen (35\%) häufig die Leitung eines Projekts übernehmen (siehe Grafik 21). 23 \% der KonsortialführerInnen stellen hochschulische Einrichtungen dar, gefolgt von außeruniversitären Einrichtungen mit $17 \%$. Non-Profit-Einrichtungen sind bei $11 \%$ der Projekte als Konsortialführung tätig und bei $9 \%$ übernehmen Kompetenzzentren die Projektleitung. 
Neben den Fördernehmerlnnen kommt vor allem (vor-)schulischen Einrichtungen eine große Bedeutung als Projektbeteiligte zu. Bei Betrachtung aller am Projekt beteiligten AkteurInnen zeigt sich laut ibw (2018), dass (vor-)schulische Bildungseinrichtungen mit $55 \%$ die bedeutendste Gruppe darstellen. Dabei sind vorwiegend Volksschulen, gefolgt von Neuen Mittelschulen / Hauptschulen und Allgemeinbildende Höhere Schulen in die Talente regional Projekte involviert.

Laut Ansicht der ExpertInnen sind unter den Einreichenden häufig Organisationen vertreten, die bereits über Erfahrung in der Antragstellung verfügen und teilweise auch entsprechende Kontakte vorweisen können. Für die Einreichung eines Talente regional Projekts ist ihrer Meinung nach viel Eigeninitiative und Engagement gefragt. Auch die Befragung des ibw (2018) zeigt auf, dass die Idee zur Beteiligung an den Ausschreibungen meist von der Konsortialführung selbst kommt. Bei der Suche nach PartnerInnen für das Konsortium werden sowohl bestehende Netzwerke aktiviert als auch neue Kontakte geknüpft. Vor allem Bildungseinrichtungen werden im Rahmen der Projekte als neue Kontakte erschlossen. Für deren Beteiligung ist das Engagement des Direktoriums und der Lehrkräfte eine wesentliche Grundvoraussetzung.

Entsprechend der Bundesländerzugehörigkeit der Konsortialführung zeigt sich, dass Talente regional Projekte im Analysezeitraum vorwiegend in Wien und in der Steiermark umgesetzt wurden. Bei jeweils einem Viertel der 141 geförderten Projekte kam die Projektleitung aus Wien oder der Steiermark. Etwas mehr als ein Zehntel der Projekte wurden in Oberösterreich, Tirol und Niederösterreich realisiert. In den anderen Bundesländern kam es nur zu vereinzelten Projekten im Rahmen von Talente regional.

Grafik 21 | Organisationsform und Bundesland der Konsortialführung, Ausschreibungen 2011-2019, in Prozent
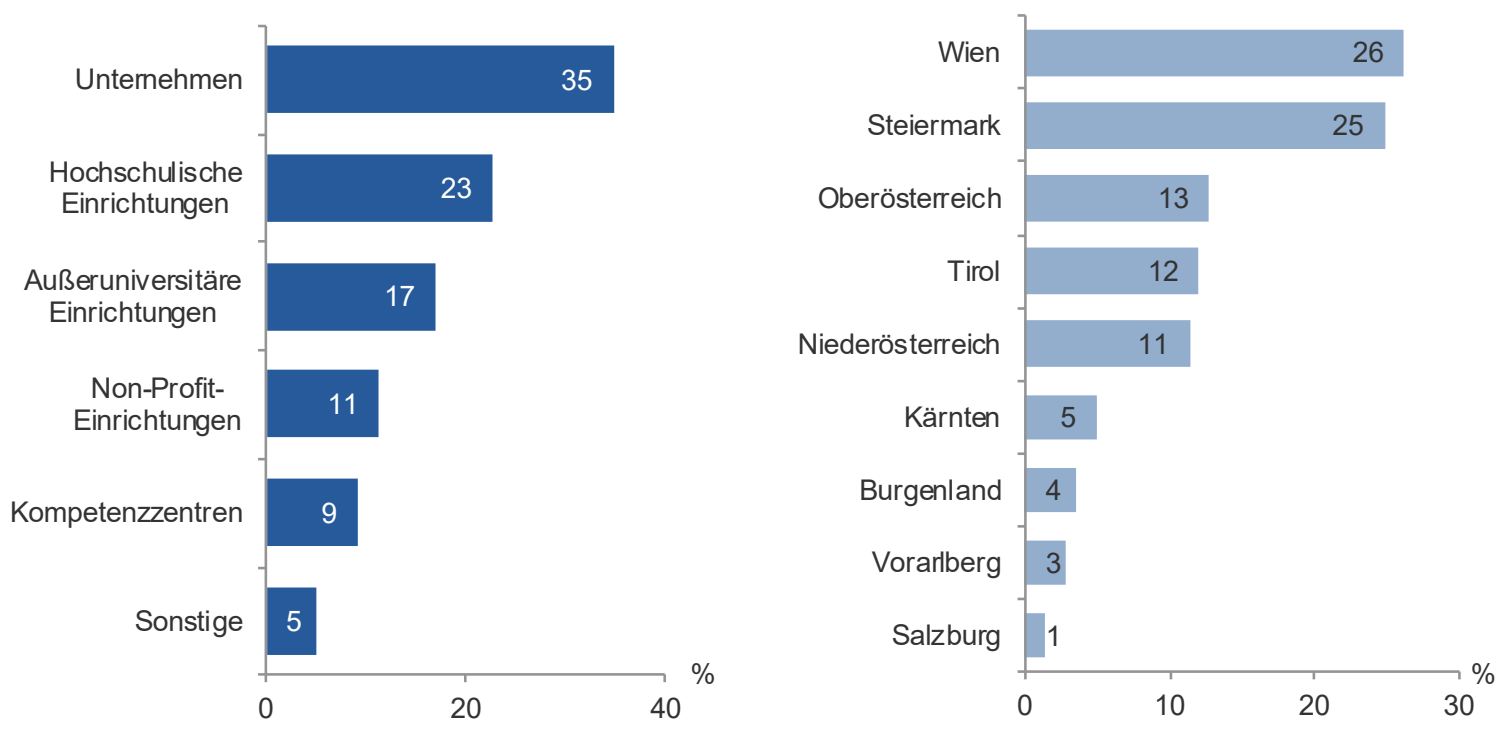
In die Talente regional Projekte sind nicht nur die verschiedenen, aktiven ProjektteilnehmerInnen aus Bildung, Wirtschaft und Wissenschaft eingebunden, es besteht darüber hinaus die Möglichkeit, für jedes geförderte Projekt noch zehn weitere Bildungseinrichtungen miteinzubeziehen. Diese erhalten Kooperationszuschüsse in Form einer Pauschalförderung von $€ 1.000$,- und können von den Aktivitäten der Projekte, z. B. durch erstellte Lehrmaterialen und konzipierte Workshops, profitieren. Damit soll die Bekanntheit der Programmlinie weiter gefördert und innovative Unterrichtsformen an Kindergärten und Schulen forciert werden.

Insbesondere bei den Ausschreibungen 2011, 2012 und 2016 fanden die Kooperationszuschüsse großen Anklang. In diesen Jahren wurden sogar mehr Anträge für Kooperationsschüsse gestellt, als Förderungen vorhanden waren. Im Zeitraum von 2012 bis 2016 lag die Anzahl der genehmigten Anträge jeweils unter dem maximalen Kontingent an Kooperationszuschüssen.

Grafik 22 | Anzahl der Kooperationszuschüsse, Ausschreibungen 2011 - 2016

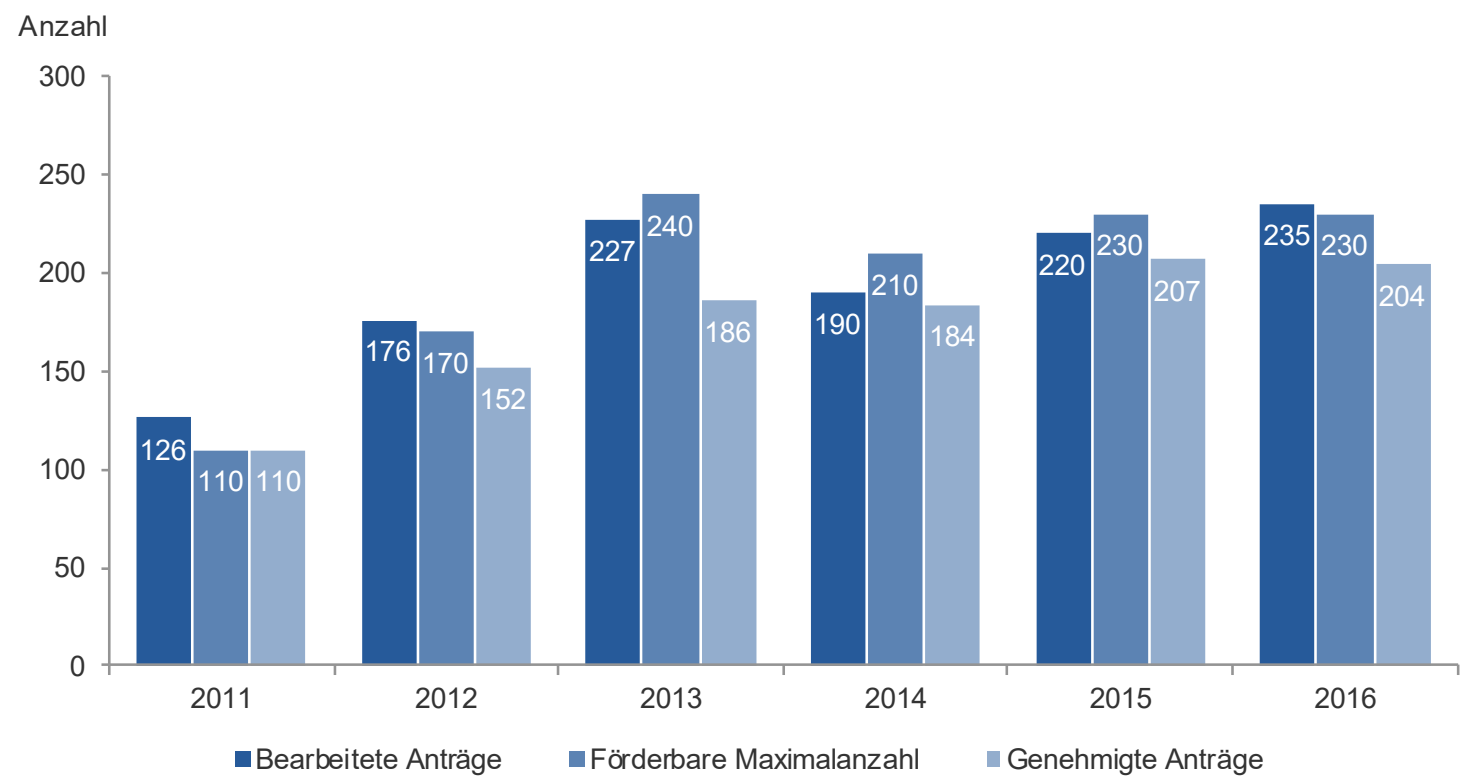

Quelle: ibw (2018): Review Talente regional, Projekt-Endberichte (2015, 2016); Berechnung und Darstellung KMU Forschung Austria

Hauptzielgruppe der Talente regional Projekte sind Kinder und Jugendlichen, die für Forschung, Naturwissenschaft und Technik begeistert werden sollen. Im Zeitraum von 2011 und 2016 konnten in den einzelnen Projekten durchschnittlich zwischen rd. 280 und rd. 450 Kinder und Jugendliche mit den konkreten Projektaktivitäten, wie Workshops und Schulungen etc., erreicht werden. Vor allem in den Jahren 2013 und 2015 konnten durchschnittlich jeweils mehr als 400 Kinder in die Aktivitäten der Talente regional Projekte eingebunden werden. Der Anteil der involvierten Mädchen und Burschen, die jeweils rd. die Hälfte der Teilnehmerlnnen bilden, ist im Zeitverlauf sehr ausgewogen. Unter Einbeziehung der weiteren Organisationen, die Kooperationszuschüsse in Anspruch nahmen, erhöht sich der Personenkreis der involvierten Kinder und Jugendlichen beträchtlich. Diese konnten vor allem durch die Teilnahme an Veranstaltungen, Ausstellungen und Ähnlichem profitieren. 
Grafik 23 | Durchschnittliche Anzahl der involvierten Kinder/Jugendlichen pro Projekt, Ausschreibungen 2011-2016

Mittelw erte

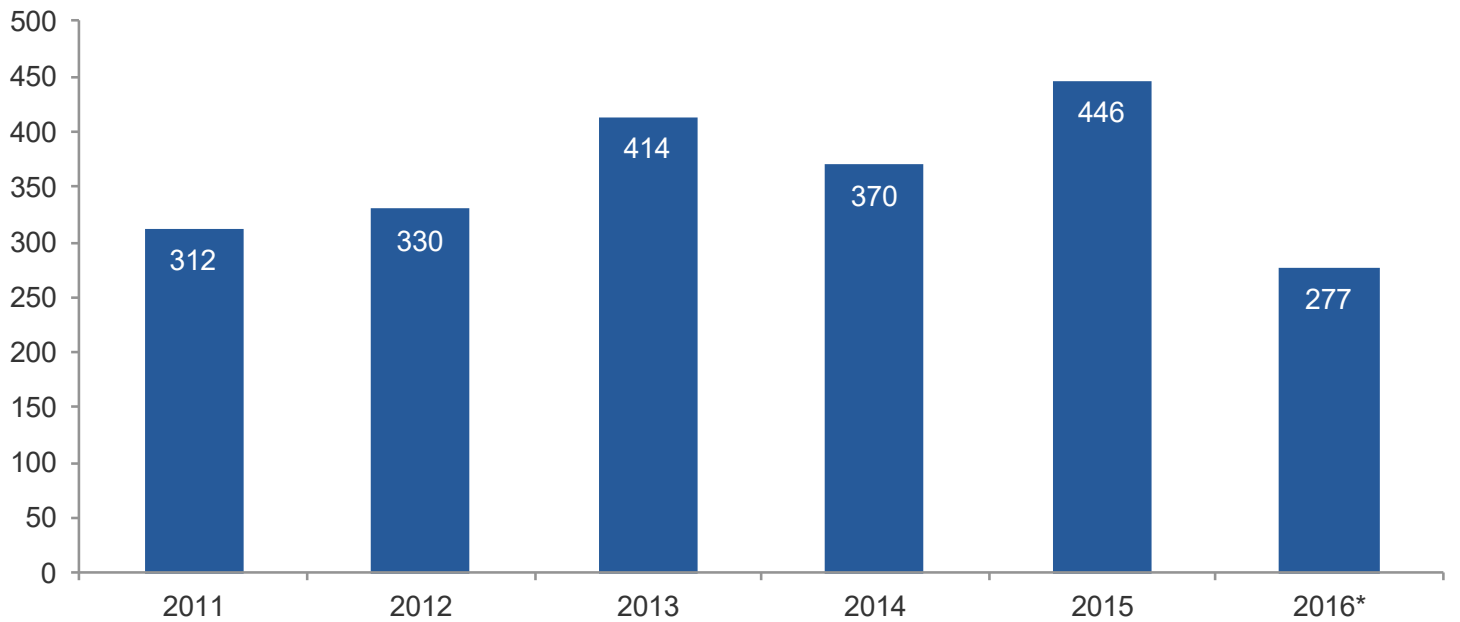

*Daten von 20 Projekten

Quelle: ibw (2018): Review Talente regional, Projekt-Endberichte (2015, 2016); Berechnung und Darstellung KMU Forschung Austria

\subsubsection{Wirkungen der Talente regional Projekte und Beurteilung der Pro- grammlinie}

\section{Ergebnisse der Zwischenevaluierung 2014}

Die Zwischenevaluierung aus dem Jahr 2014 (vgl. Heckl et al. 2014) zeigt bereits, dass die Programmlinie Talente regional ein sehr geeignetes Instrument darstellt, um Kinder und Jugendliche für Naturwissenschaft und Technik zu begeistern, ihre Kenntnisse in diesen Bereichen zu erweitern und Einblicke in zukunftsträchtige Berufsfelder zu erhalten. Als Stärke der Programmlinie werden die Offenheit bezüglich der Themen und Herangehensweisen gesehen sowie die Langfristigkeit der Projekte. Von großer Relevanz sind auch die hohe Praxisorientierung sowie die zahlreichen Interaktionsmöglichkeiten von ForscherInnen und Kindern / Jugendlichen. Aber nicht nur die Kinder und Jugendlichen, sondern auch die verschiedenen ProjektpartnerInnen profitierten von der Teilnahme. Die wissenschaftlichen PartnerInnen konnten ihre Tätigkeiten einem breiteren Publikum vermitteln und ihre regionale Bekanntheit steigern, die beteiligten UnternehmerInnen profitierten von langfristigen Kontakten zu potentiellen Nachwuchskräften und die PädagogInnen erweiterten ihre Kenntnisse, insbesondere auch im methodischen Bereich, und konnten auf ein umfangreicheres Materialportfolio zurückgreifen. Daher wurde die Talente regional Programmlinie von den befragten ProjektteilnehmerInnen nicht nur weiterempfohlen, sondern meist auch ein neues Projekt mit einem ähnlichen Projektkonsortium eingereicht. 
Optimierungspotential wird in der aufwändigen Koordination der kooperativen Projekte gesehen, da alle Befragten davon berichteten, dass der Aufwand in keinem Verhältnis zur Förderung stehe. Daher könnte zum Beispiel dem Projektmanagement ein höherer Budgetanteil zugeordnet werden. Auch hinsichtlich der Kooperationszuschüsse wurde eine Flexibilisierung des Instruments angeregt.

Inhaltlich liegt die Herausforderung der Talente regional Projekte in der zielgruppenadäquaten Aufbereitung der Themen, Materialien, Experimente etc. für die verschiedenen Altersstufen, da die Einbindung von drei Bildungsstufen verpflichtend vorgesehen war. Es wurde daher empfohlen, dass nur zwei Bildungsstufen verpflichtend abdeckt werden müssen. Zur Optimierung der Integration der Projekte in den Schulalltag, sollte der Zeitpunkt der Ausschreibung so gewählt werden, dass diese bei der Planung des neuen Schuljahres bereits berücksichtigt werden können. Diese Empfehlungen wurden umgesetzt. Da die Talente regional Projekte mit sehr viel Entwicklungsarbeit verbunden sind, wurde auch angeregt, erfolgreiche Projekte mit ähnlichem Projektinhalt in einer neuerlichen Ausschreibung zuzulassen, wenn sie auf andere Regionen übertragen oder andere BildungspartnerInnen eingebunden werden.

\section{Ergebnisse des Reviews der Talente regional Projekte 2018}

Im Jahr 2018 führte das Institut für Bildungsforschung der Wirtschaft (ibw) eine umfassende Analyse der 135 Talente regional Projekte im Zeitraum von 2010 bis 2016 durch (Gruber / Schmid 2018), die auch eine quantitative Befragung der ProjektteilnehmerInnen enthielt. Im Rahmen des Reviews wurden zu 112 Projekten Einzeldarstellungen erstellt und auf der open4innovation Website veröffentlicht. Als Hauptwirkungen der Projektaktivitäten wurde dabei hervorgehoben, dass vor allem die Kinder und Jugendlichen von den Talente regional Projekten durch die Erweiterung ihrer Kenntnisse in Naturwissenschaft und Technik profitieren konnten und ihr Interesse an diesen Bereichen gestiegen ist. Auch ein realitätsnaher Einblick in die Tätigkeit von Technikerlnnen und ForscherInnen wurde innen ermöglicht. Die Projektteilnehmerlnnen berichteten als positive Effekte vor allem von der Begeisterung und dem Forschungsgeist, der bei den Kindern und Jugendlichen geweckt wurde sowie vom Stolz und gestärkten Selbstbewusstsein der Zielgruppe. Ein Erfolgsfaktor der Talente regional Projekte ist das Projektdesign, das sich durch aktive Mitarbeit, eine abwechslungsreiche Aufbereitung der Inhalte sowie ein offenes Setting auszeichnet. Auch die Exkursionen und Workshops stellen einen nicht zu unterschätzenden Erlebnisfaktor dar.

Die involvierten PädagogInnen profitierten vor allem dadurch, dass sie neue Unterrichtsmethoden kennenlernten, realitätsnahe Einblicke in Unternehmen bekamen und Projektarbeiten verstärkt in den Unterricht integrierten. Auf der Ebene der Schulen kam es zu einer Stärkung der schulinternen Zusammenarbeit und einer Steigerung des Stellenwerts von Naturwissenschaft und Technik im Unterricht. Bei etwa der Hälfte der Befragten Bildungseinrichtungen wurde außerdem die technische Infrastruktur an der Schule verbessert und regionale Kooperationen mit Wirtschaft und Forschung auf- und ausgebaut.

Ein wichtiges Ziel der Talente regional Projekte stellt das Fördern nachhaltiger regionaler Vernetzungen von Bildung, Wissenschaft und Wirtschaft dar. Dies wurde insofern erreicht, als $72 \%$ der 
befragten Projektteilnehmerlnnen angaben, dass nach Projektabschluss nachhaltige Kooperationen mit einzelnen Organisationen entstanden sind.

Insgesamt wird die Programmlinie Talente regional von den befragten Projektbeteiligten als besonders gut geeignet wahrgenommen, um das Interesse von Kindern und Jugendlichen an Forschung, Technologie und Innovation zu steigern und den Bezug zu Naturwissenschaft und Technik durch ihre aktive Einbindung zu vertiefen. Kritikpunkte an der Gestaltung der Talente regional Projekte betreffen den großen Rahmen der Projekte, so dass viele Ressourcen in Organisation, Koordination und Dokumentation fließen. Schwierigkeiten bereiten einerseits das Projekt- und Zeitmanagement (Koordinierung von Terminen, Projektkommunikation) sowie die konkrete Projektkonzeption, wobei vor allem das gendersensible Design und die altersgerechte Vermittlung Herausforderungen darstellen. Auch die methodische Umsetzung der Aktivitäten und die starke aktive Einbindung der Kinder und Jugendlichen wurde als anspruchsvoll erachtet. Für den Erfolg der Aktivitäten nehmen die Bildungseinrichtungen eine zentrale Rolle ein. Daher spielt die möglichst frühe Einbindung der PädagogInnen in die Konzeption der Projektaktivitäten eine besonders wichtige Rolle.

\section{Wirkungsanalyse}

Talente regional wird von den beiden Vorgängerstudien als auch von Seiten der befragten Expertlnnen attestiert, eine international einzigartige, sehr ambitionierte Programmlinie zu sein, die Kinder und Jugendliche in vielen Altersgruppen für Wissenschaft und Forschung begeistert. Eine wesentliche Stärke ist die Themenvielfalt der Projekte, die unterschiedlichste naturwissenschaftliche und technische Schwerpunkte setzen. Auch der Erlebnisfaktor durch Experimente, Workshops oder persönliche Begegnungen mit ForscherInnen bietet die Möglichkeit, das Interesse in eine gewisse Richtung zu lenken, insbesondere von jenen, die bereits eine gewisse Affinität zu diesen Themen haben. Um ein breites Interesse zu wecken, ist es wichtig, die Projektaktivitäten stark an den Lebensrealitäten der Kinder und Jugendlichen auszurichten.

Eine weitere Besonderheit der Talente regional Projekte stellt die Erprobung neuer pädagogischer Konzepte und innovativer Unterrichtsmethoden (z. B. Peer und Tutorial Learnings) dar. In der Folge führt dies zu einer verstärkten Integration zeitgemäßer Materialen, Methoden und innovativer Unterrichtskonzepte in den regulären Schulalltag. Um dies zu gewährleisten und langfristig sicherzustellen, ist es wichtig, die PädagogInnen möglichst frühzeitig in die Planung und Konzeption der Projektaktivitäten miteinzubeziehen und dem Lehrpersonal projektspezifische Fortbildungen zu ermöglichen. Auch die Lehrenden müssen laut den befragten ExpertInnen den Umgang mit neuen Technologien und technischen Innovationen in einer angenehmen Lernumgebung erlernen, damit sie ihr praktisches Wissen an die SchülerInnen weitergeben können. Dabei ist gezielte Technikdidaktik gefragt, um auch nicht-technik-affine Lehrkräfte dafür zu begeistern. Für eine erfolgreiche Implementierung und nachhaltige Fortführung ist auch wesentlich, dass im schulischen Altag mit einem relativ strikten Organisationskonzept Freiräume für die Durchführung projektbezogener Aktivitäten geschaffen werden.

Ein weiterer bedeutender, nachhaltiger Effekt der Talente regional Projekte besteht in der Vernetzung und der Einbindung möglichst vieler regionaler AkteurInnen. Die Kooperationszuschüsse tragen dazu bei, den Wirkungskreis der Talente regional Projekte zu vergrößern, weil weitere 
Bildungseinrichtungen in der regionalen Umgebung angesprochen werden und die regionale Bekanntheit der Projekte steigt. Die beteiligten Bildungseinrichtungen profitieren von der finanziellen Unterstützung in erster Linie, indem sie ihre Infrastruktur (Geräte, Materialen) an den Bildungseinrichtungen ausbauen und verstärkt an Exkursionen oder Besichtigungen der regionalen ProjektpartnerInnen teilnehmen. Die mit Hilfe der Talente regional Projekte initiierten Kooperationen zwischen Schulen, Forschungseinrichtungen und Unternehmen können für die Umsetzung weiterer Projekte hilfreich sein. Die befragten ExpertInnen beobachten, dass Schulen, die einmal mitgewirkt haben, sich immer wieder an der Programmlinie beteiligen. Manche Projekte fungieren praktisch als Leuchtturm für weitere und nachhaltige Initiativen in diesem Bereich. In diesem Zusammenhang wurden sogar schon eigene Vereine gegründet, um die Projektideen weiter fortzusetzen. Ein Beispiel dafür ist der Verein klasse!Forschung in Tirol, der Bildungseinrichtungen mit PartnerInnen aus Wissenschaft und Wirtschaft bei der Umsetzung von Bildungsprojekten vernetzt und aktiv unterstützt. Die Aktivitäten sind an die Lehrpläne angepasst und sollen dazu beitragen, dass in Schulen mehr geforscht und experimentiert wird.

Kritik wird hingegen hinsichtlich des beträchtlichen Organisationsaufwands bei der Koordination der verschiedensten involvierten AkteurInnen geäußert. Die befragten ExpertInnen weisen darauf hin, dass Bildungseinrichtungen mit der Abwicklung solcher Forschungsprojekte kaum vertraut sind. Auch dass sich die Projekte über mehrere Altersgruppen erstrecken, wird wie bereits in der Evaluierung 2014 (Heckl et al.) als sehr anspruchsvoll wahrgenommen. In der Praxis wird vor allem die Einbindung der Kindergärten als herausfordernd erlebt, da für diese Zielgruppe ganz andere pädagogische Konzepte erforderlich sind. Dadurch verlieren die Projekte manchmal an Tiefe. Eine Alternative wäre die Projektzusammensetzung auf gewisse Altersgruppen, Schultypen oder -standorte zu konzentrieren. Aber gerade in der Kooperation von Primär-, Sekundärund Tertiärstufe und dem Zusammenführen der unterschiedlichen Altersgruppen wird auch eine Win-Win-Situation für alle Beteiligten gesehen.

Die starke Fokussierung auf den Innovationsgrad der Projekte ist für die praktische Umsetzung und Aufbereitung für die Zielgruppe der Kinder und Jugendlichen nicht immer leicht. Der Innovationsanspruch wird in dieser Projektkonstellation als sehr ambitioniert empfunden. Es müssten nicht immer neue Projekte erfunden werden, sondern könnten auch bereits erprobte Konzepte in anderen Schulen oder Regionen zum Einsatz kommen. Hier könnten insbesondere ländliche Regionen profitieren, in denen seltener vergleichbare Initiativen gestartet werden. Im ländlichen Bereich müssen oftmals erst die Strukturen für solche Projekte geschaffen werden.

Das komplexe Projektdesign mit den zusätzlichen Schwerpunkten der Geschlechterausgewogenheit sowie der Integration von Kindern und Jugendlichen unabhängig ihrer sozialen oder geographischen Herkunft führt bei den ProjektteilnehmerInnen teilweise zu Verunsicherungen, inwiefern diese Themen in die Aktivitäten integriert werden können. Laut Ansicht der befragten ExpertInnen, ist es jedoch sehr wichtig, diese Zielgruppen durch gezielte Auswahl entsprechender Schulen und Schulklassen anzusprechen und nicht einzelne, interessierte Kinder zu adressieren. Über die Schule haben benachteiligte Kinder oft erst die Chance, Forschung, Naturwissenschaft und Technik etwas näher kennenzulernen, da im privaten Rahmen weniger Initiativen wie Museumsbesuche, Workshop-Teilnahmen etc., gesetzt werden. Um die Zielgruppen möglichst nachhaltig für diese Themen zu begeistern, wäre es laut ExpertInnenmeinung wichtig, auch 
die Eltern und das soziale Umfeld stärker einzubeziehen, da diese maßgeblichen Einfluss auf die weiteren Schul- und Berufsentscheidungen haben. Wenn die Projekte das naturwissenschaftliche und technische Interesse der Kinder und Jugendlichen wecken, kann dieses sehr rasch durch das soziale Umfeld wieder zunichtegemacht werden.

Zur Steigerung der Bekanntheit der Talente regional Programmlinie, wäre es wichtig, erfolgreiche Projekte als Good Practices verstärkt medial zu präsentieren. Dafür könnten Projekte herausgegriffen werden, die maßgebliche Änderungen an den Schulen, wie z. B. Flächenbegrünungen, oder in der regionalen Umgebung realisiert haben, um die Aktivitäten möglichst anschaulich zu präsentieren. Das steigert die Bekanntheit des gesamten Programms und regt interessierte Organisationen an, dafür einzureichen. Um vor allem PädagogInnen über diese innovative Fördermöglichkeit und hilfreiche Unterstützung aufmerksam zu machen, könnten verstärkt Vorträge, Sensibilisierungs- und Informationsaktivitäten an pädagogischen Hochschulen initiiert werden. Auch der Austausch mit den bisherigen Projektteilnehmerlnnen könnte noch stärker forciert werden, um auf den Erfahrungen der anderen aufbauen zu können.

Um die Nachhaltigkeit und den Erfolg der Talente regional Projekte weiter auszubauen, wäre es laut ExpertInnenmeinung zielführend, besonders erfolgreiche Projekte, die viele Kinder begeistert haben, mit einer zusätzlichen Förderung auszustatten, um eine längerfristige Fortsetzung zu ermöglichen. Auch die Unternehmen könnten z. B. durch das Angebot verstärkter medialer Präsenz motiviert werden, ihr Engagement nicht nur auf zwei Jahre, sondern auf längere Zeiträume auszudehnen und Veranstaltungen in diesem Bereich fortzusetzen. 


\section{3 | FEMtech Praktika für Studentinnen}

Die Programmlinie FEMtech Praktika für Studentinnen unterstützt Nachwuchswissenschaftlerinnen in der angewandten Forschung in Naturwissenschaft und Technik Fuß zu fassen. Im Rahmen der bis zu 6 Monate dauernden Praktika lernen die Studentinnen berufliche Ein- und Aufstiegswege kennen und erhalten einen Einblick in die angewandte Forschung.

Tabelle 3 | Eckpunkte zu FEMtech Praktika für Studentinnen

\begin{tabular}{|c|c|}
\hline $\begin{array}{l}\text { FEMtech Praktika für Stu- } \\
\text { dentinnen }\end{array}$ & Kurzbeschreibung \\
\hline Ziele & $\begin{array}{l}\text { Frauen für eine naturwissenschaftlich-technische Berufsentscheidung zu } \\
\text { motivieren und den Anteil von Frauen sowie ihrer Karrierechancen in den } \\
\text { geförderten Organisationen zu erhöhen }\end{array}$ \\
\hline Zielgruppen & $\begin{array}{l}\text { Unternehmen, außeruniversitäre Forschungseinrichtungen, Technologie- } \\
\text { transfer-Einrichtungen, InnovationsmittlerInnen etc. sowie sonstige nicht- } \\
\text { wirtschaftliche Einrichtungen (Fördernehmerlnnen) } \\
\text { Studentinnen und Nachwuchswissenschaftlerinnen }\end{array}$ \\
\hline Inhalt & $\begin{array}{l}\text { Förderung von hochwertigen Praktikumsplätzen für weibliche Studierende in } \\
\text { Unternehmen und außeruniversitären Forschungseinrichtungen im natur- } \\
\text { wissenschaftlich-technischen Bereich. Dabei steht die Vermittlung praxisbe- } \\
\text { zogenen Know-hows und das Heranführung an die angewandte Forschung } \\
\text { im Vordergrund. }\end{array}$ \\
\hline Einreichverfahren & $\begin{array}{l}\text { Laufende Einreichung nach Ausschreibung, bei Mittelausschöpfung vorzei- } \\
\text { tiges Schließen der Ausschreibung } \\
2011 \text { - } 2012 \text { (Oktober } 2011 \text { bis Juni 2012) } \\
2012 \text { - } 2013 \text { (September } 2012 \text { bis Dezember 2013) } \\
2014,2015 \text { (jeweils Mai - Juli) } \\
\text { 2016, 2017, } 2018 \text { (jeweils Mai - September) } \\
2019 \text { (März - Mai und Oktober) }\end{array}$ \\
\hline Laufzeit & $\begin{array}{l}\text { Praktikumsdauer: } 1 \text { Monat }-6 \text { Monate mit einem Beschäftigungsausmaß } \\
\text { von mind. } 28,5 \text { Wochenstunden }\end{array}$ \\
\hline Förderhöhe & $\begin{array}{l}2011-2013: € 2.100,- \text { bis } € 10.600,- \text { je nach Dauer des Praktikums } \\
\text { Ab 2014: } € 1.680 \text {,- bis } € 8.480 \text {,- je nach Dauer des Praktikums }\end{array}$ \\
\hline Spezifika & 2013/2014: Themenschwerpunkt Mobilität der Zukunft \\
\hline
\end{tabular}

\subsection{1 | Struktur der geförderten Praktikumsplätze für Studentinnen}

Im Zeitraum von 2011 bis 2019 wurde für rd. 3.600 Praktikumsplätze für Studentinnen eine finanzielle Unterstützung genehmigt. Von einer finanziellen Förderung konnten 2011 bis 20183.066 Praktikantinnen profitieren, für 2019 wurde für 533 Praktikumsstellen eine Förderung zugesagt, die tatsächliche Anzahl der geförderten Praktikumsplätze steht jedoch noch nicht fest.

Die Anzahl der geförderten Praktikumsplätze ist im Zeitverlauf kontinuierlich gestiegen. Im Jahr 2012/2013 fand nur eine Ausschreibung für zwei Jahre statt. Dabei wurden die Mittel für die Ausschreibung 2012 zu einem weiteren Termin aus dem Budget für die Ausschreibung 2013 aufgestockt, wodurch eine eigene Ausschreibung für das Jahr 2013 entfiel. Die meisten Praktikums- 
plätze, nämlich 579, wurden 2018 gefördert, für 2019 reduziert sich die Anzahl der für die Förderung genehmigten Praktikumsstellen leicht. Dies ist aber nicht auf einen geringeren Mitteleinsatz zurückzuführen, sondern auf die durchschnittliche Praktikumsdauer, die bei den genehmigten Praktika für 2019 über denen der geförderten Praktika 2018 liegt. So hängt die Anzahl der geförderten Praktika nicht nur von der Höhe der Fördermittel ab, sondern auch von der Praktikumsdauer, durch die die Höhe der Förderung je Praktikum bestimmt wird.

Grafik 24 | Anzahl der geförderten FEMtech Praktika für Studentinnen nach Ausschreibung, 2011-2019

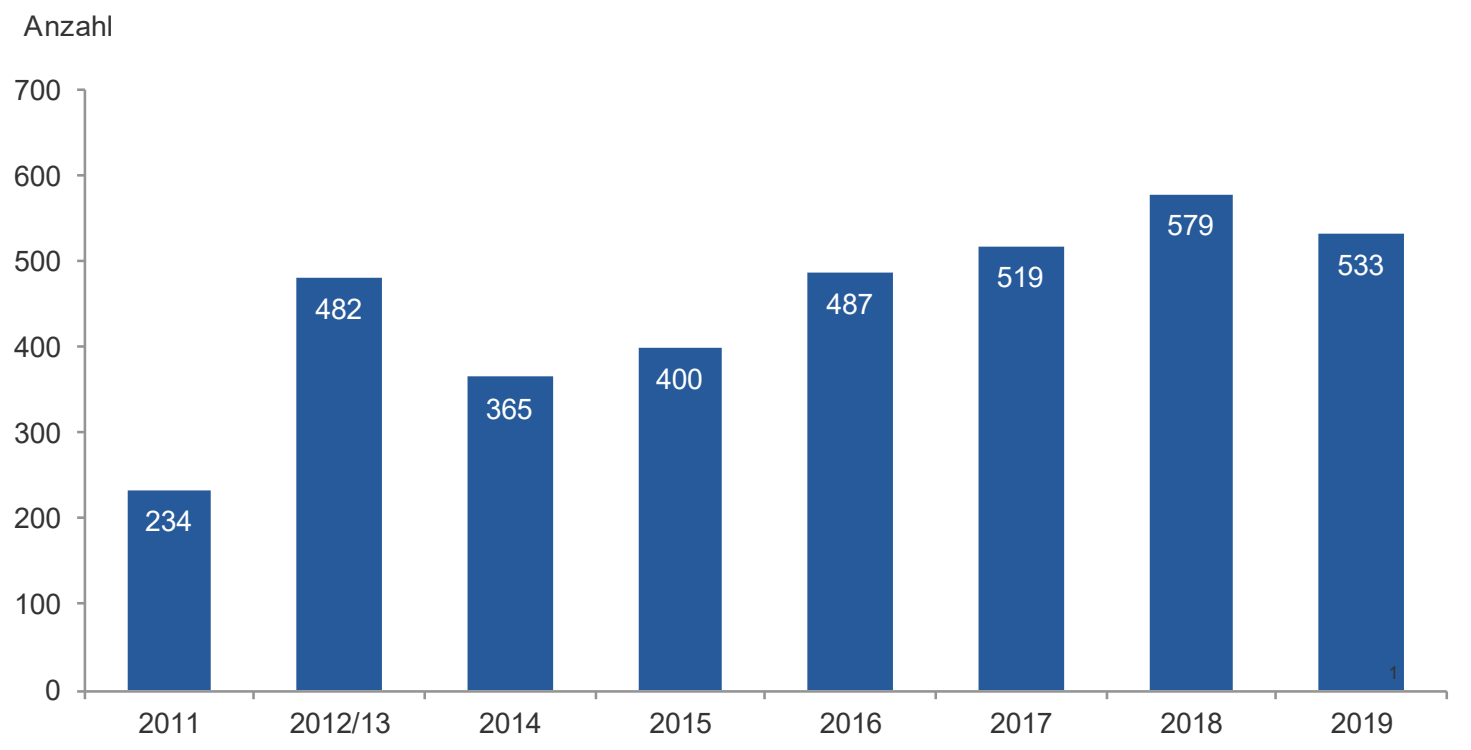

Quelle: FFG; Berechnung und Darstellung KMU Forschung Austria

2019: 533 genehmigte Praktika, die tatsächliche Anzahl der geförderten Praktika steht für 2019 noch nicht fest

Rd. zwei Drittel der Praktika für Studentinnen im Zeitraum von 2011 bis 2019 fanden/finden im Bereich der Naturwissenschaften statt und ein Drittel entfällt auf technische Wissenschaften. Ein etwas höherer Anteil technischer Wissenschaften wurde im Jahr 2016 mit 37 \% erreicht (siehe Grafik 25).

Auch in Hinblick auf die verschiedenen Themenfelder der Praktika zeigt sich, dass neben sonstigen Themenbereichen insbesondere die Life Sciences mit 26 \% im Vordergrund stehen. 
Grafik 25 | Wissenschaftsdisziplinen und Themenfelder der geförderten FEMtech Praktika für Studentinnen, Ausschreibungen 2011-2019, in Prozent

\section{Wissenschaftsdisziplinen 2011-} 2019

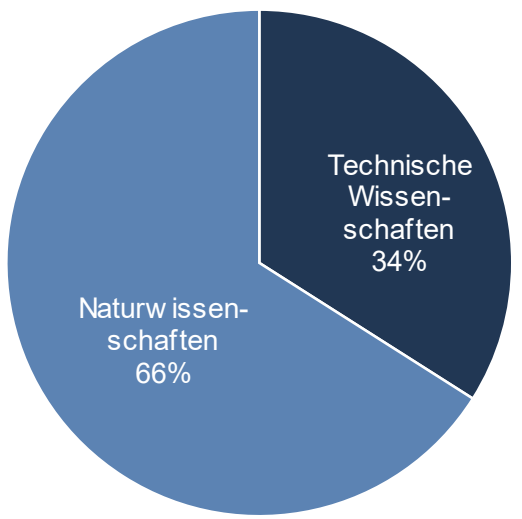

Themen der Praktika, 2011-2019

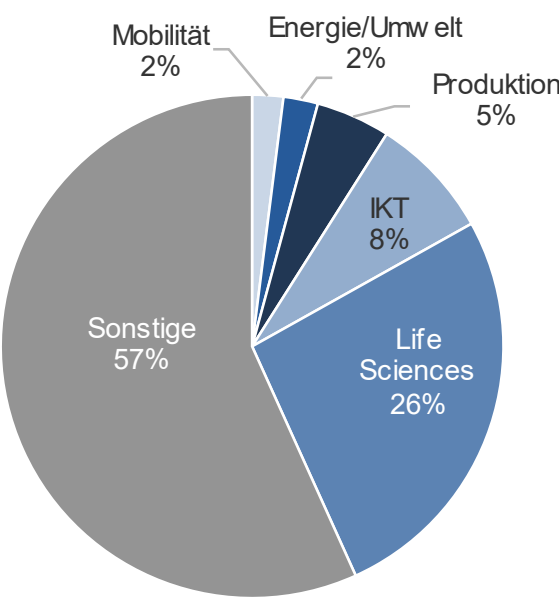

Quelle: FFG, FFG FEMtech Praktika für Studentinnen: Statistik genehmigte Praktika 2019; Berechnung und Darstellung KMU Forschung Austria

Hinsichtlich der Praktikumsdauer zeigt sich, dass sowohl Nachfrage nach kürzeren als auch nach längeren Praktika besteht (siehe Grafik 26). Am beliebtesten sind sechsmonatige Praktika. Zwischen $33 \%$ und $49 \%$ der Praktika erstrecken sich im Zeitraum von 2011 bis 2019 über 6 Monate. Auch kurze Praktikumsphasen von einem und zwei Monaten werden häufig absolviert, denn bis zu einem Viertel aller Praktika konzentrieren sich nur auf einen Monat und rd. ein Fünftel werden in zwei Monaten durchgeführt. Im Durchschnitt dauern die Praktika drei bis vier Monate (Mittelwerte von 3,4 bis 4,2 Monate). Die Praktika werden gerne in den Sommermonaten absolviert. 2018 fanden z. B. 46 \% der geförderten Praktika von Juli bis September statt. 
Grafik 26 | Dauer der geförderten Praktika, Ausschreibungen 2011-2019, in Prozent

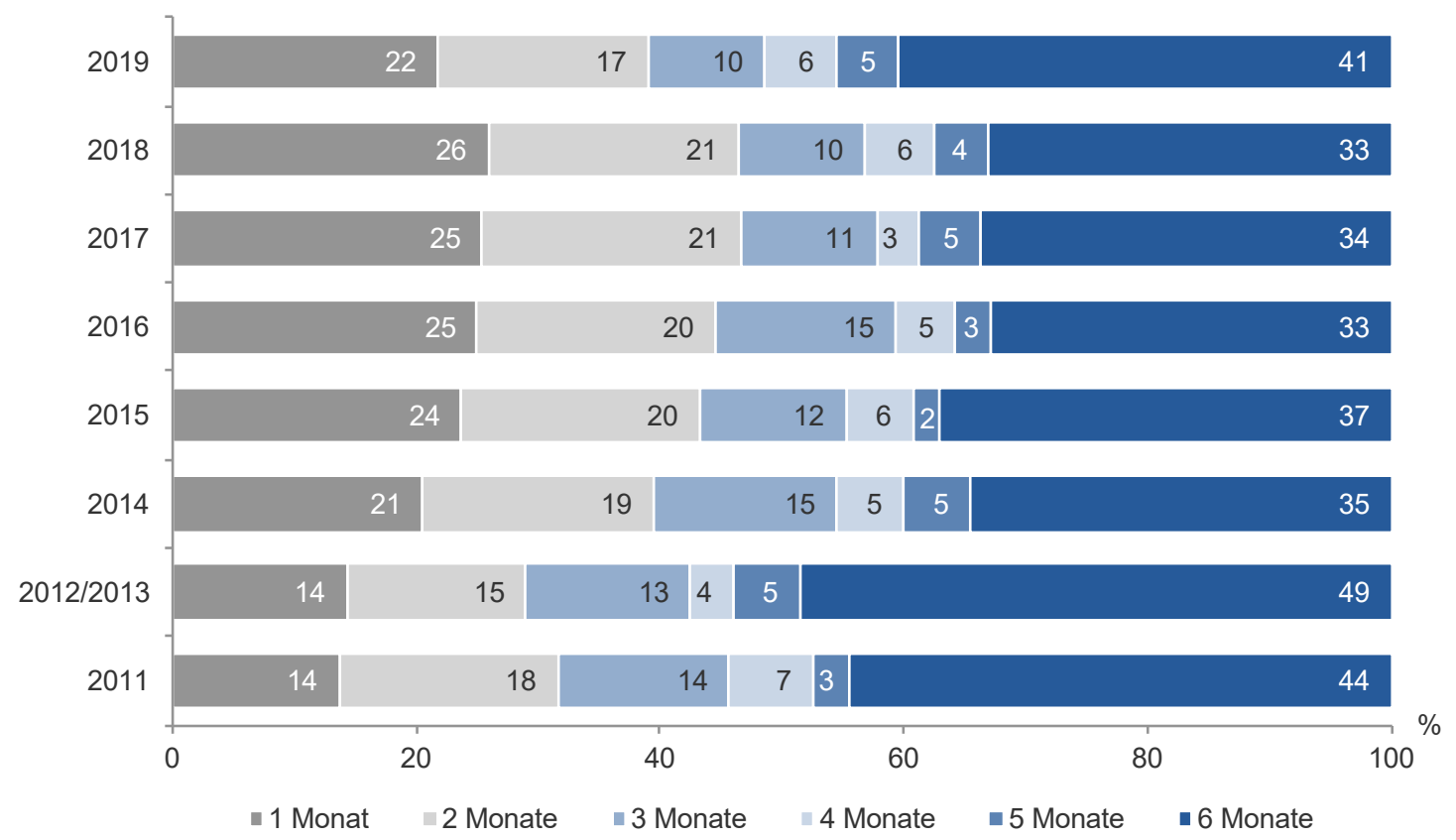

Quelle: FFG FEMtech Praktika für Studentinnen Schlussauswertungen 2011-2018, Statistik genehmigte Praktika 2019; Darstellung KMU Forschung Austria

Die meisten Praktika werden im Zeitraum 2011 bis 2019 in Wien durchgeführt, gefolgt von Niederösterreich und der Steiermark.

Grafik 27 | Geförderte Praktika für Studentinnen nach Bundesländern, Ausschreibungen 2011-2019, in Prozent

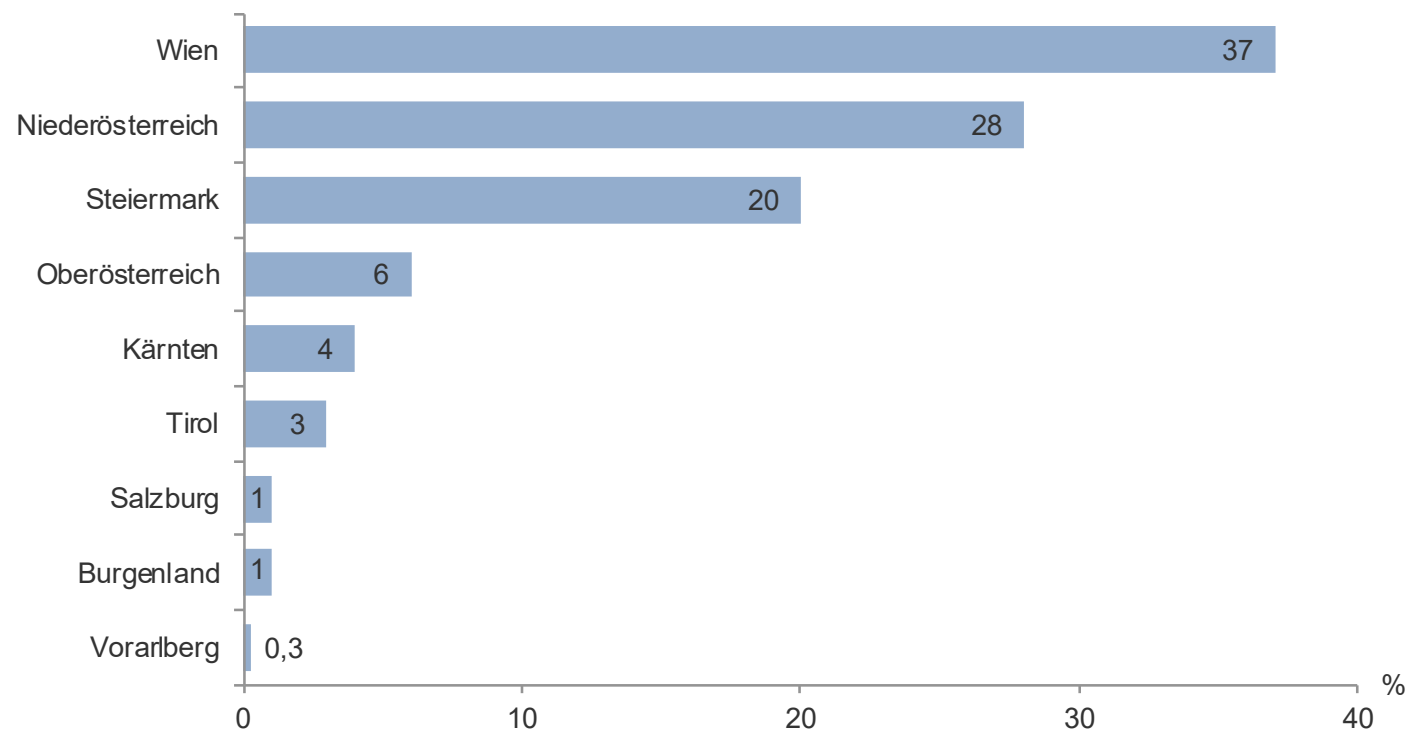

Quelle: FFG FEMtech Praktika für Studentinnen: Statistik genehmigte Praktika 2019; Darstellung KMU Forschung Austria 
Die Mehrheit der geförderten Praktikumsstellen wird in außeruniversitären Forschungseinrichtungen (58 \%) angeboten. $42 \%$ der finanziell unterstützten Praktika finden in Unternehmen statt und dabei vor allem in KMU (25\%).

Grafik 28 | Praktika nach Organisationsart, Ausschreibungen 2011-2019, in Prozent

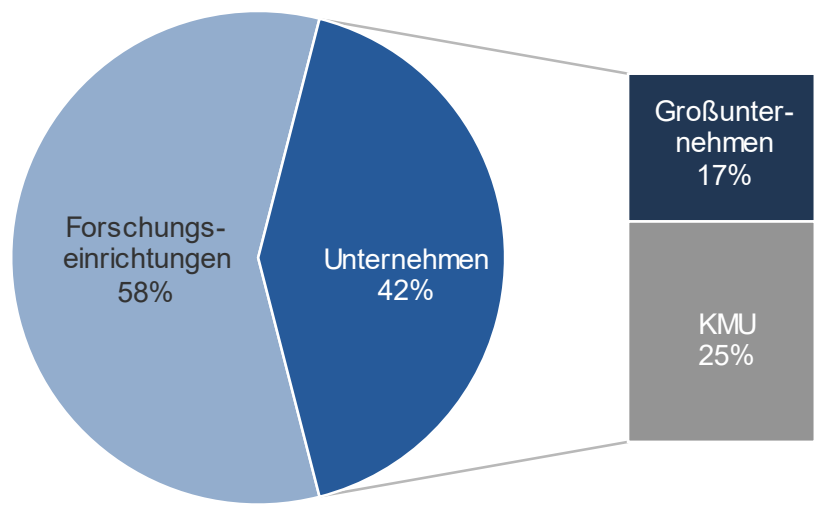

Quelle: FFG FEMtech Praktika für Studentinnen: Statistik genehmigte Praktika 2019; Darstellung KMU Forschung Austria

Die Analyse der teilnehmenden Organisationen ohne Berücksichtigung der Anzahl der jeweils angebotenen Praktikumsplätze zeigt, dass vor allem Unternehmen (66 \%) zu den FördernehmerInnen zählen. Davon können insbesondere KMU (51 \%) profitieren, während nur 15 \% Großunternehmen eine finanzielle Unterstützung erhalten. Die Forschungseinrichtungen machen hingegen nur ein Drittel (34 \%) der FördernehmerInnen aus, stellen jedoch häufig mehrere Praktikumsplätze für Studentinnen zur Verfügung.

Eine Analyse der Anzahl der geförderten Praktika nach Organisationstyp in den Jahren 2019, 2018 und 2017 zeigt, dass jeweils rd. 60 \% der beteiligten Organisationen ein gefördertes Praktikum anbieten (siehe Grafik 29). Vor allem Unternehmen stellen für Studentinnen großteils nur einen Praktikumsplatz zur Verfügung. Die überwiegende Mehrheit der Unternehmen (96 \% bzw. $94 \%$ ) bietet bis zu 4 Praktikumsmöglichkeiten an. Die Forschungseinrichtungen nehmen hingegen oftmals mehrere Studentinnen gleichzeitig als Praktikantinnen auf. Diese Tendenz wird im Zeitverlauf von 2017 bis 2019 immer ausgeprägter, wobei insbesondere der Anteil der Forschungseinrichtungen mit mehr als 10 PraktikantInnen von $6 \%$ auf $12 \%$ steigt. Zudem ist die Konzentration bei einzelnen AnbieterInnen ersichtlich: so bot die/der Top-Praktikumsanbieterln in den Jahren 2017 bis 2019 jeweils rd. 100 Praktikumsplätze an, auf den Plätzen folgen Organisationen mit rd. 30 bis 40 Praktika für Studentinnen. 
Grafik 29 | Anzahl der Praktika nach Organisationstyp, Ausschreibungen 2017, 2018 und 2019, in Prozent

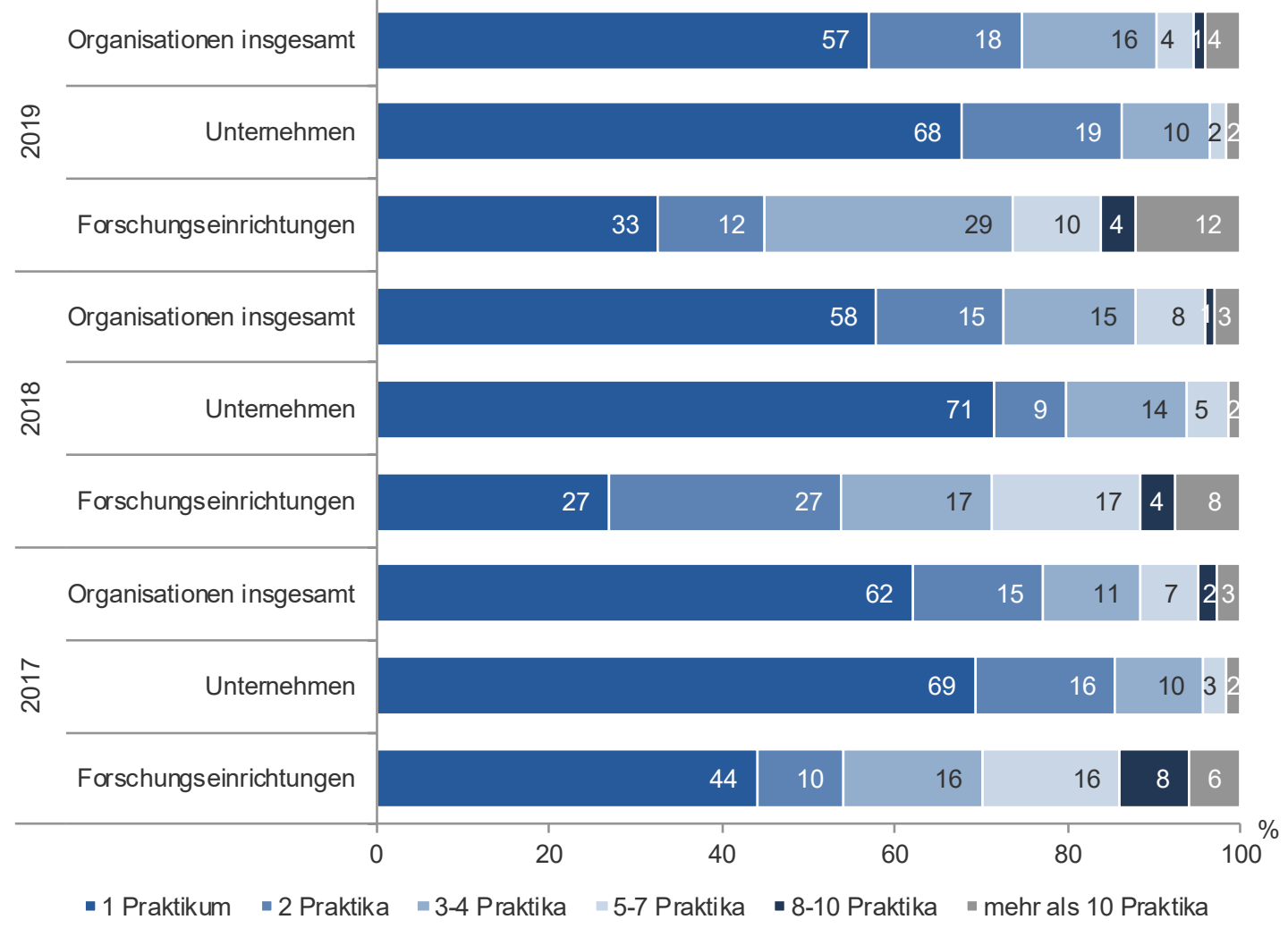

Quelle: FFG; Berechnung und Darstellung KMU Forschung Austria

\subsection{2 | Charakteristika der Studentinnen}

Der Großteil der Praktika wird von Universitätsstudentinnen absolviert, im Jahr 2018 trifft dies beispielsweise auf $87 \%$ zu. $13 \%$ der Praktikantinnen besuchten 2018 eine Fachhochschule. ${ }^{3}$ Vor allem Studentinnen der Universität für Bodenkultur, der Technischen Universität Wien und der Universität Wien nutzten die FEMtech Praktika im Jahr 2018. Um mehr FH-Studentinnen zu gewinnen, wurden bei der Ausschreibung 2019 bereits Mobilisierungsaktivitäten im Bereich der Fachhochschulen (z. B. durch gezielte Teilnahmen an Karrieremessen der FHs) gesetzt.

Die Praktika werden von den Studentinnen grundsätzlich in jeder Phase des Studiums (Bachelor und Master) genutzt. Die Entwicklung der durchschnittlichen Semesteranzahl der Praktikantinnen zeigt eine Tendenz zur späteren Nutzung des geförderten Praktikumsangebots im Studienverlauf. Während die Studentinnen in den Jahren 2011-2013 das Praktikum durchschnittlich im 6. oder 7. Semester absolvierten, machen die Studentinnen im Jahr 2019 im Durchschnitt erst im 9. Semester davon Gebrauch, wie Grafik 30 zeigt.

\footnotetext{
${ }^{3}$ Diese Information wird erst seit der Ausschreibung 2016 erhoben. 2016 und 2017 sind die Werte ähnlich
} 
Grafik 30 | Durchschnittliche Semesteranzahl bei den geförderten Praktika, Ausschreibungen 2011-2019

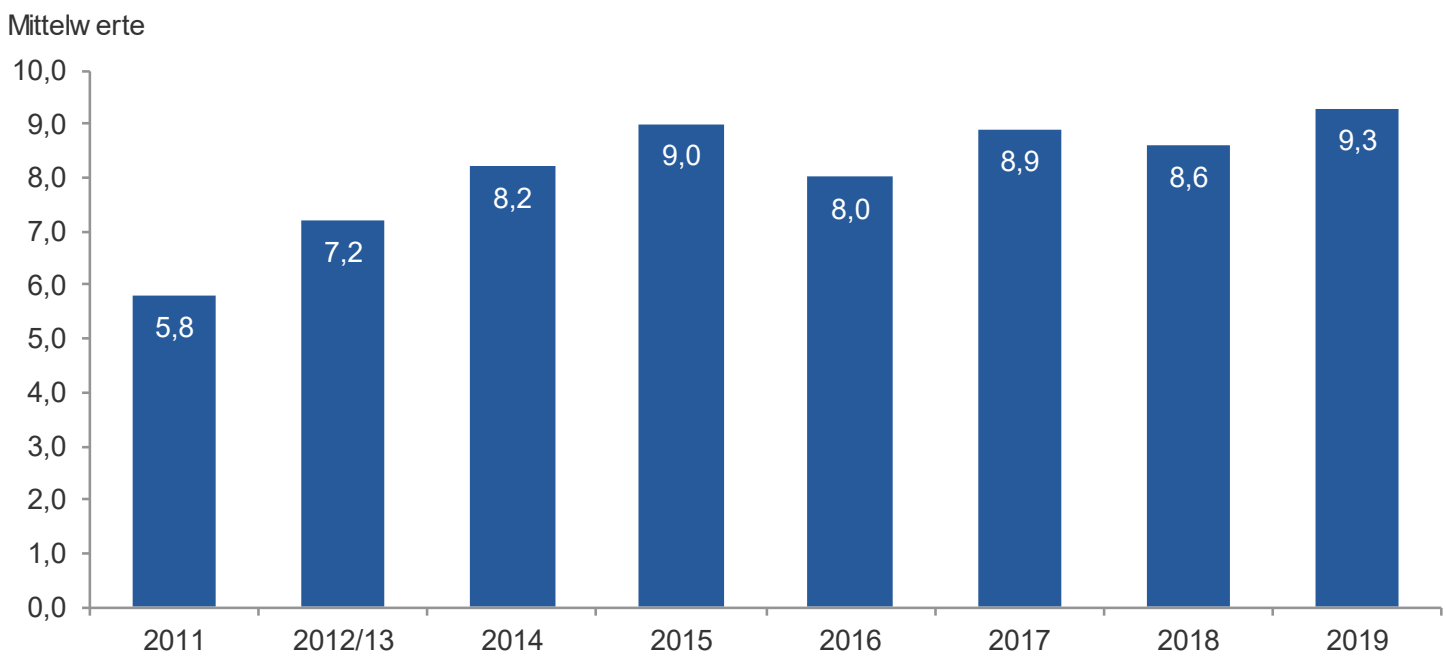

Quelle: FFG FEMtech Praktika für Studentinnen Schlussauswertungen 2011-2018, Statistik genehmigte Praktika 2019; Darstellung KMU Forschung Austria

Die Praktikantinnen haben laut eigener Auskunft von der Möglichkeit eines förderbaren Praktikums in erster Linie von ihren ArbeitgeberInnen erfahren. Weitere wichtige Informationsquellen sind das persönliche Umfeld der Studentinnen, die Universität oder Fachhochschule sowie das Internet. Während ArbeitgeberInnen und Universitäten / Fachhochschulen als Informationsquellen im Zeitverlauf etwas an Bedeutung verlieren, informieren sich immer mehr Studentinnen im Internet über das geförderte Praktikumsangebot.

Grafik 31 | Wichtige Informationsquellen über die Möglichkeit eines förderbaren Praktikums für die Praktikantinnen, Ausschreibungen 2011-2018 in Prozent (Mehrfachnennungen möglich)

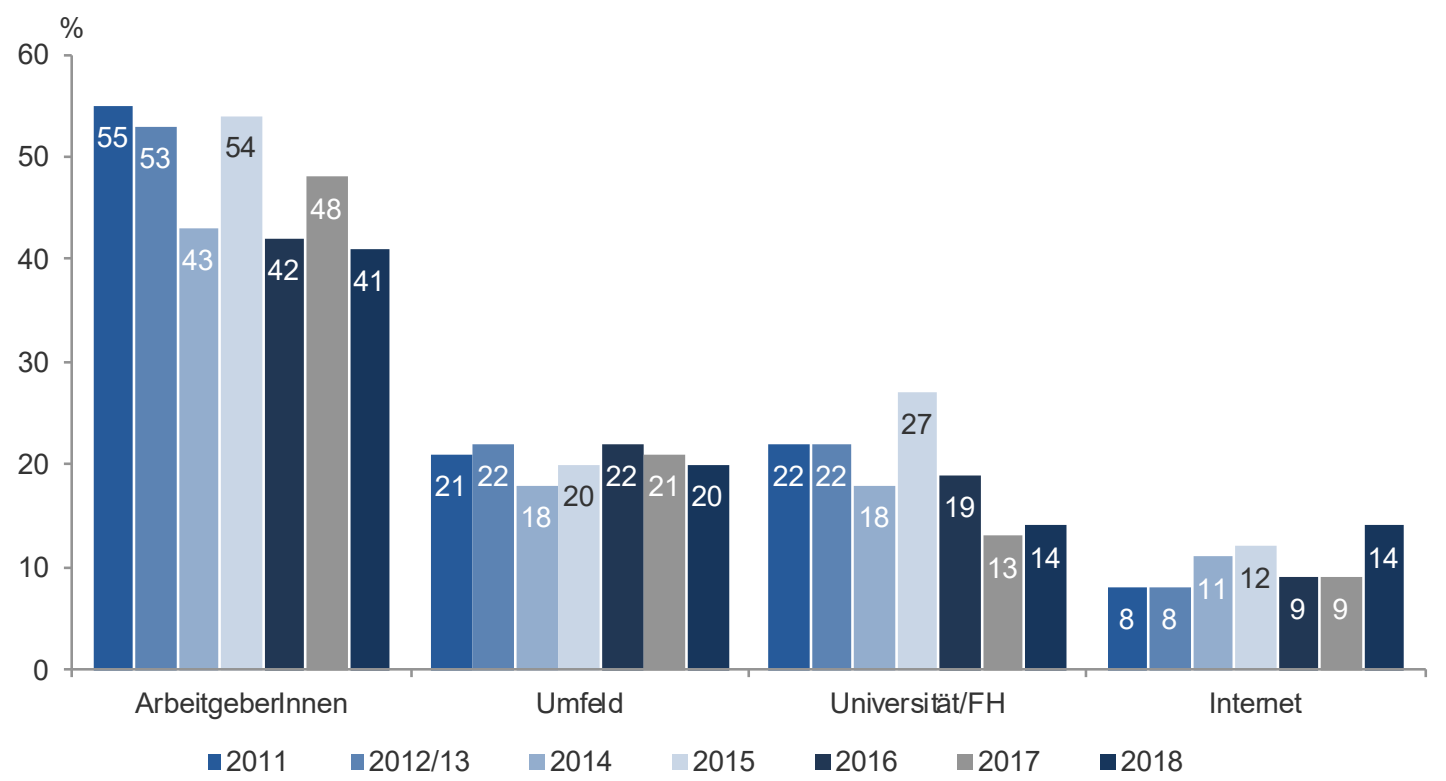

Quelle: FFG FEMtech Praktika für Studentinnen Schlussauswertungen 2011-2018; Darstellung KMU Forschung Austria 


\subsubsection{Beurteilung und Wirkungen der FEMtech Praktika für Studentinnen}

Die Teilnahme an der Programmlinie FEMtech Praktika für Studentinnen führt zu einem steigenden Interesse der teilnehmenden Organisationen, Studentinnen aktiv zu fördern. Vor allem in den Jahren 2011 bis 2014 wiesen mehr als 90 \% der Fördernehmerlnnen auf ein gestiegenes Interesse hin, dieser Wert verringerte sich danach, lag aber 2018 noch immer bei $71 \%$.

Es bietet einen Anreiz, aktiv nach Studentinnen für Aufgaben im F\&E zu suchen.

Es motiviert, verstärkt Studentinnen einzusetzen und kann so unterstützen, den Anteil von Frauen in technischen Berufen zu erhöhen.

Insgesamt nehmen in den Jahren 2011 bis $201865 \%$ bis $55 \%$ der FördernehmerInnen einen unmittelbaren Nutzen der FEMtech Praktika für Studentinnen wahr (siehe Grafik 32). Die Unternehmen und Forschungseinrichtungen profitieren insbesondere von den neuen Ideen und Sichtweisen der Studentinnen.

Frische Ideen und jugendliches Hinterfragen haben die Forschungsarbeit bereichert.

Für die Betriebe ist es sehr von Vorteil, dass die Praktikantinnen neue Sichtweisen und Ideen in die Projekte einbringen.

Für beide Parteien ist der Erfahrungsaustausch extrem gut und die Synergien können genutzt werden.

Darüber hinaus schreiben 66 \% bis 75 \% der FördernehmerInnen in den Jahren 2011 bis 2018 den Praktika einen langfristigen Nutzen zu, im Jahr 2018 sind es $69 \%$. Dieser besteht in erster Linie in der Win-Win-Situation für Studentinnen und Unternehmen bzw. Forschungseinrichtungen. Studentinnen haben die Möglichkeit, in ihr Berufsfeld hinein zu schnuppern und Berufserfahrungen zu sammeln und gleichzeitig profitieren die Unternehmen / Forschungseinrichtungen, indem sie potentielle Mitarbeiterinnen vorab kennenlernen können. Dadurch wird auch längerfristig der Einstieg von Frauen in Forschung und Technik erleichtert und weiter forciert.

Unterstützt Studentinnen, Praxis zu sammeln und mehr Selbstbewusstsein zu bekommen sowie Unternehmen beim Finden von zukünftigen High Potentials.

FEM-Tech Praktika sind die ideale Gelegenheit, um zukünftiges Personal kennenzulernen und die Frauenquote im Unternehmen zu erhöhen.

Studentinnen werden motiviert, ihren Weg in der Technik weiter zu verfolgen. So kann die Anzahl der Frauen in der Technik mittelfristig gesteigert werden. 
Grafik 32 | Beurteilung des Nutzens der Praktika für Studentinnen für die Fördernehmerlnnen, Ausschreibungen 2011-2018, in Prozent (Mehrfachnennungen möglich)

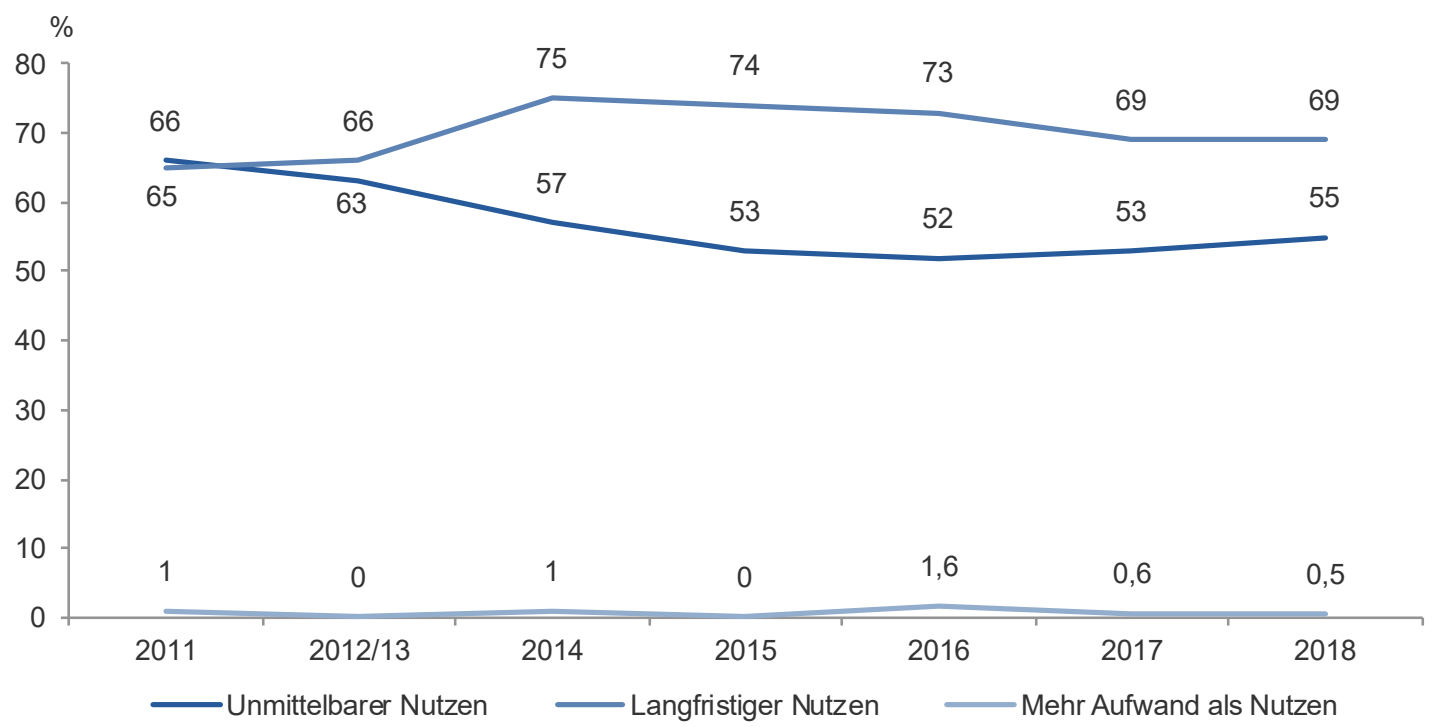

Quelle: FFG FEMtech Praktika für Studentinnen Schlussauswertungen 2011-2018; Darstellung KMU Forschung Austria

Aufgrund der positiven Erfahrungen will der Großteil der Fördernehmerlnnen auch in Zukunft Praktikumsplätze für Studentinnen anbieten, wobei $201885 \%$ sicherlich und $13 \%$ dies wahrscheinlich vorhaben. In den Jahren davor wird das zukünftige Angebot von Praktikumsmöglichkeiten ähnlich hoch eingeschätzt.

Auch die Studentinnen sind mit den geförderten Praktika sehr zufrieden, wie im Zeitraum 2011 bis 2018 jeweils rd. zwei Drittel angeben (siehe Grafik 33). Eine (sehr) gute Beurteilung gaben im Jahr 2014 sogar 90 \% ab, 2018 traf dies auf 83 \% zu. Die Praktika boten den Studentinnen viele Lernmöglichkeiten sowie spannende Erfahrungen und Einblicke in Forschung und Technik.

Das Praktikum hat sich gelohnt, weil ...

ich eine tolle und lehrreiche Erfahrung gemacht habe, von der ich auch in Zukunft profitieren werde.

ich einen Einblick in die angewandte Forschung bekommen habe, den ich durch das Studium so nicht bekomme. 
Grafik 33 | Beurteilung der Praktika durch die Studentinnen, Ausschreibungen 2011-2018, in Prozent

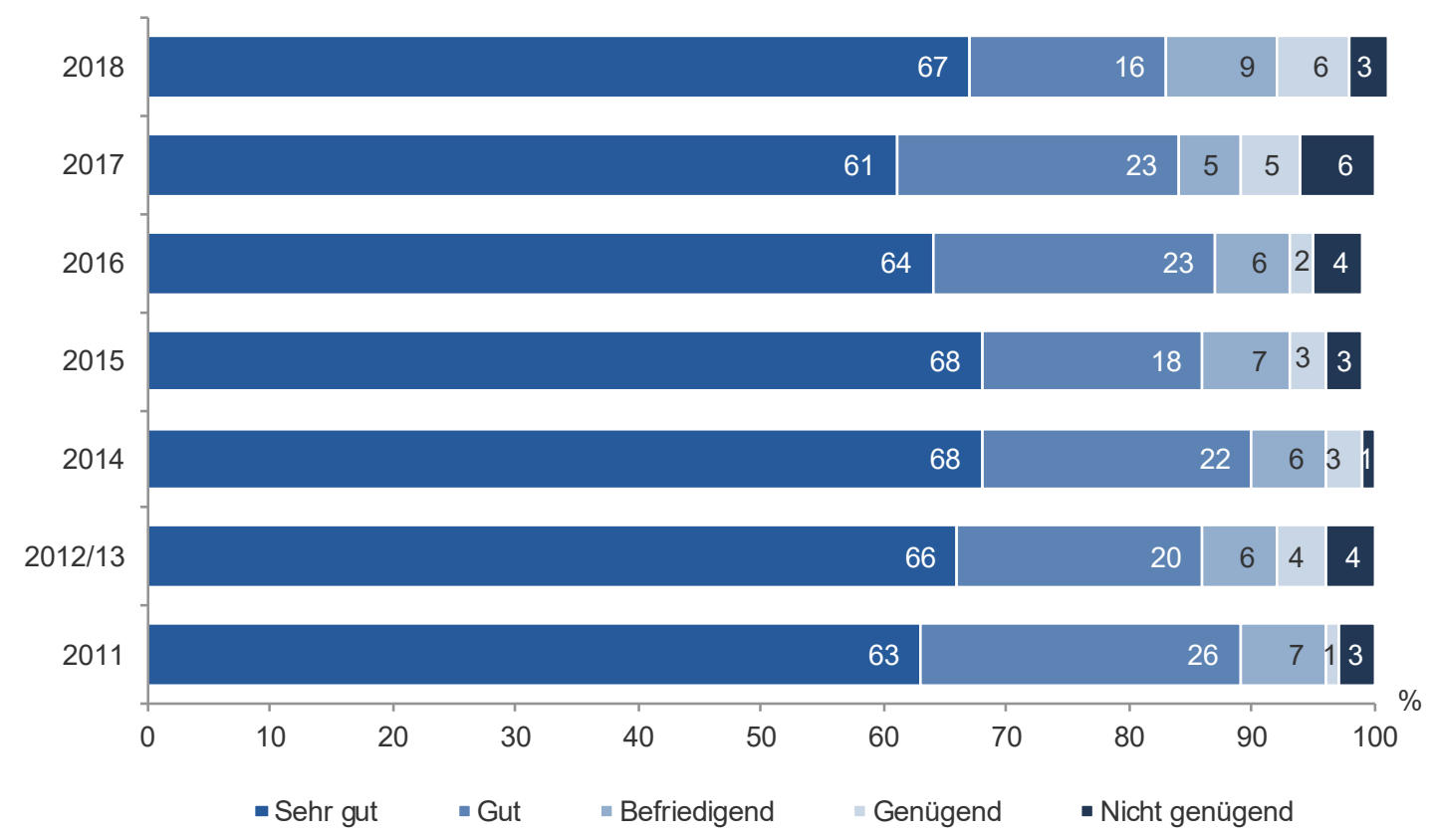

Quelle: FFG FEMtech Praktika für Studentinnen Schlussauswertungen 2011-2018; Darstellung KMU Forschung Austria

Der Hauptgrund für die Durchführung eines Praktikums ist für die Studentinnen das Sammeln von Berufserfahrung. Dies konnte nach Angaben der Studentinnen im Jahr 2018 auch erreicht werden, denn $97 \%$ haben berufliche Erfahrungen gesammelt und $82 \%$ Kontakte geknüpft, wie Grafik 34 zeigt.

Das Praktikum hat sich gelohnt, weil ...

ich einen Einblick in die tägliche Arbeit von Forscherlnnen bekommen habe und direkt an einem spannenden Projekt mitarbeiten konnte.

ich praktischen Einblick in einen technischen Beruf bekommen habe und viele neue Kontakte knüpfen konnte.

Auch von den befragten ExpertInnen werden die Praktika als ein tolles arbeitsmarktpolitisches Instrument gesehen, da die Betriebe zukünftige Arbeitskräfte austesten und die Studentinnen gleichzeitig konkrete Berufspraxis erwerben können. Die Praktika fungieren oftmals als eine gute Einstiegsmöglichkeit für die Studentinnen, um längerfristig im Unternehmen zu verbleiben. 
Grafik 34 | Gründe und Wirkungen der Praktika für die Studentinnen, Ausschreibung 2018 in Prozent (Mehrfachnennungen möglich)

\section{Grund für Praktikum 2018}

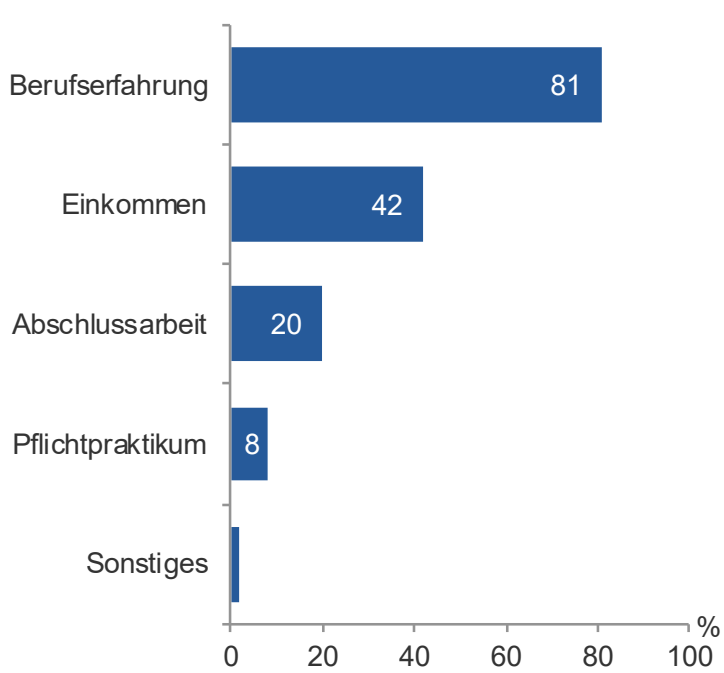

2018: Durch mein Praktikum konnte ich

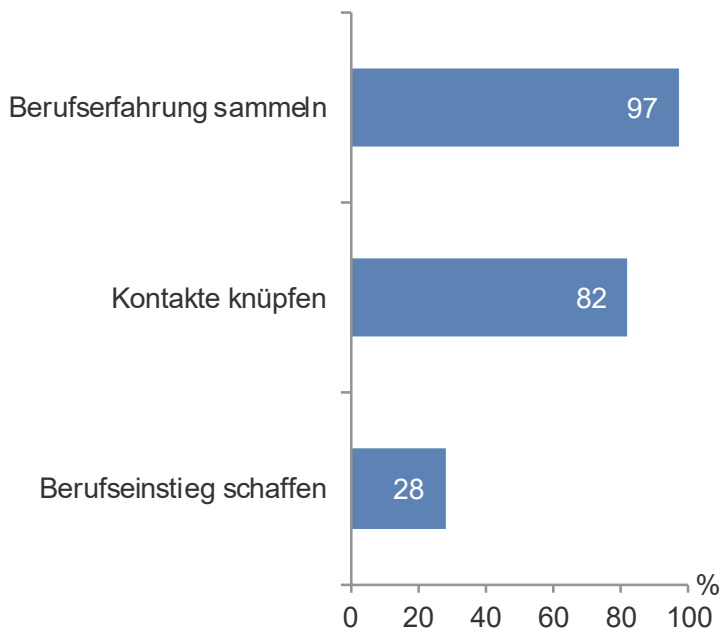

Quelle: FFG FEMtech Praktika für Studentinnen Schlussauswertung 2018; Darstellung KMU Forschung Austria

Jeweils rd. drei Viertel der Studentinnen, die im Zeitraum von 2011 bis 2018 ein gefördertes Praktikum absolviert haben, berichten, dass ihr Interesse an einer Tätigkeit in Forschung, Naturwissenschaft und Technik durch das Praktikum (stark) gestiegen ist.

FemTech Praktika sind eine tolle Idee! Ich wäre sonst nie darauf gekommen, dass mich Laborarbeit und Forschung so interessiert und mir Freude macht. 
Grafik 35 | Einschätzung des Interesses an einer Tätigkeit in Forschung, Naturwissenschaft und Technik durch die Studentinnen, Ausschreibungen 2011-2018, in Prozent

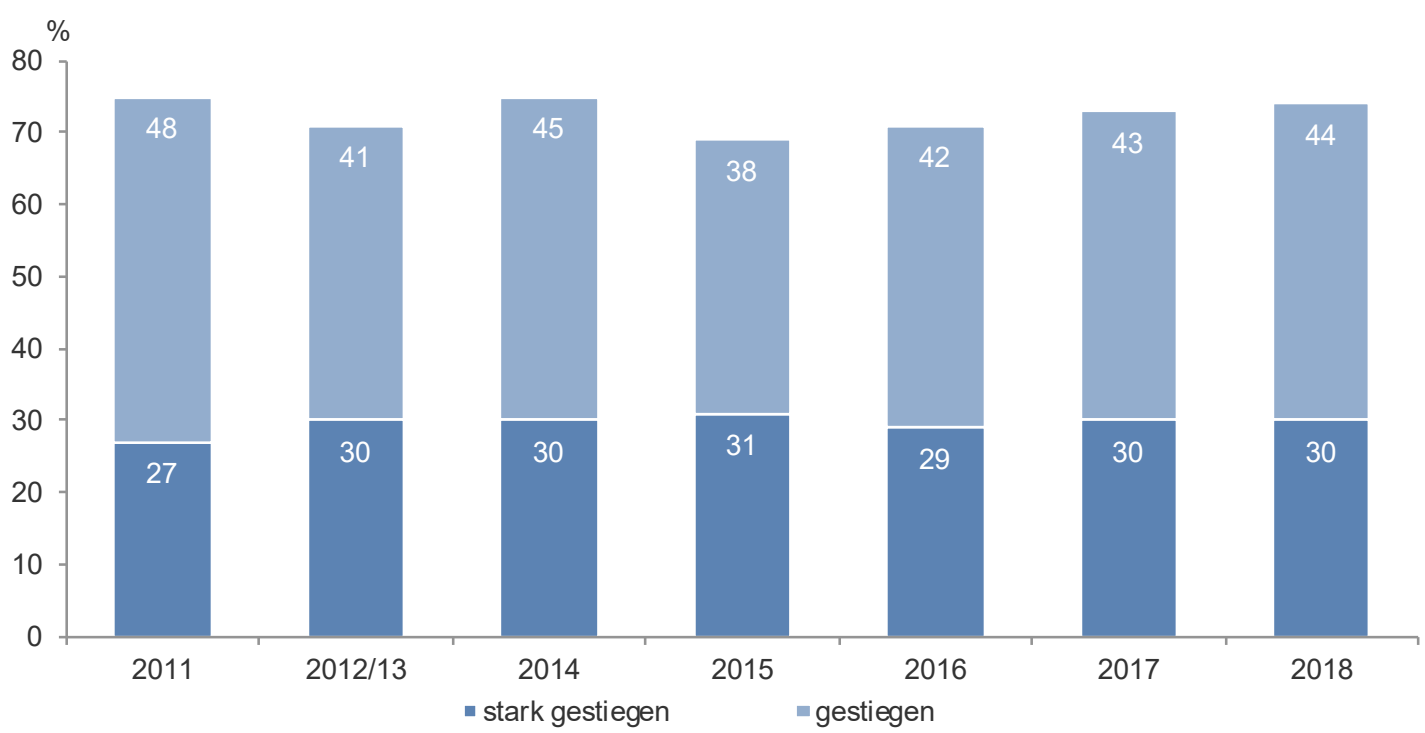

Quelle: FFG FEMtech Praktika für Studentinnen Schlussauswertungen 2011-2018; Darstellung KMU Forschung Austria

Die Praktika erleichtern den Studentinnen auch maßgeblich den Berufseinstieg. Laut Auskunft der FördernehmerInnen in den Endberichten konnten im Jahr 2018 bereits 23 \% der Studentinnen im Anschluss an die Praktikumszeit in der jeweiligen Organisation weiterbeschäftigt werden. Der Anteil der Weiterbeschäftigungen ist damit im Vergleich zum Jahr 2014, wo nur bei $7 \%$ der Praktikantinnen eine weitere Zusammenarbeit angestrebt wurde, deutlich gestiegen (siehe Grafik 36). Die geförderten Praktika stellen somit für die teilnehmenden Organisationen eine immer wichtigere Option dar, um zukünftige Arbeitskräfte zu akquirieren.

Viele unserer FEMtech-PraktikantInnen werden nach Abschluss des Praktikums in ein fixes Dienstverhältnis übernommen, was unsere Gender Balance deutlich verbessert.

Auch für die Studentinnen werden die Praktika immer relevanter, um den Berufseinstieg zu schaffen. 2018 gaben 28 \% der Praktikantinnen an, dass sie nach ihrem Praktikum ein Dienstverhältnis anschließen konnten, 2014 traf dies erst auf $14 \%$ zu. Die geförderten Praktika erleichtern den Sprung in die Arbeitswelt und helfen den Studentinnen auch längerfristig im Bereich von Forschung, Naturwissenschaft und Technik beruflich Fuß zu fassen.

Das Praktikum hat sich gelohnt, weil ...

ich dadurch eine Festanstellung bei einem tollen Arbeitgeber erhalten haben.

Ich finde die Möglichkeit des FEMtech Praktika für Studentinnen sehr hilfreich um den Einstieg in technische Berufe zu erleichtern oder überhaupt zu ermöglichen. Da Frauen in dieser Berufssparte nicht leicht eine Möglichkeit bekommen, ihr Können zu zeigen. 


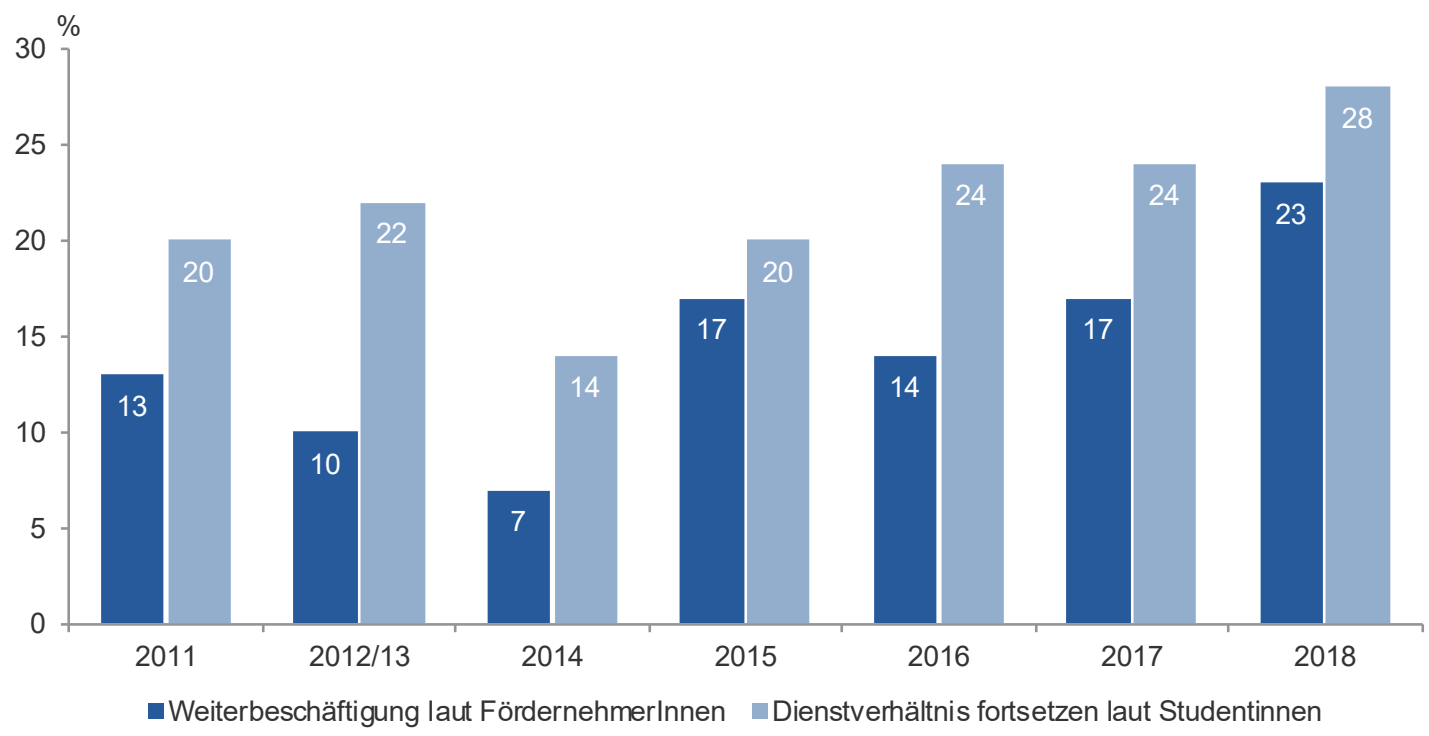

Quelle: FFG FEMtech Praktika für Studentinnen Schlussauswertungen 2011-2018

Insgesamt betrachtet unterstützen die FEMtech Praktika die berufliche Integration der Studentinnen und tragen so dazu bei, dass sich der Frauenanteil in den teilnehmenden Unternehmen und Forschungseinrichtungen erhöht. Sowohl von Seiten der Fördernehmerlnnen als auch von Seiten der Studentinnen herrscht große Zufriedenheit mit diesem Förderangebot. Dennoch werden vereinzelt Verbesserungswünsche geäußert. Diese betreffen beispielsweise die Gestaltung der Ausschreibung, wobei ein früherer Start, die Ausweitung des Ausschreibungszeitraumes oder ein zweiter Termin gewünscht wird. Auch bei der Gestaltung der Praktika wird vereinzelt mehr Flexibilität gewünscht, da vielfältige Wochenstunden-Modelle die Vereinbarkeit des Praktikums mit dem Studium erleichtern könnten. Einige dieser Anregungen wurden bereits bei den letzten Ausschreibungen aufgegriffen. Bei der Ausschreibung 2018 durften die Praktika bis Ende Juli 2019 durchgeführt werden und 2019 konnte die Ausschreibung aufgrund höherer Budgetmittel in zwei Phasen abgewickelt werden, wobei die erste bereits im März statt wie bisher im Mai startete und die zweite im Oktober erfolgte.

Ein weiterer Wunsch, der von einzelnen FördernehmerInnen und Studentinnen genannt wird, betrifft die Ausweitung des Programms auf männliche Studierende. In diesem Zusammenhang wird berichtet, dass Organisationen gezielt nach Studentinnen suchen, um die Förderung in Anspruch nehmen zu können. Männliche Praktikanten werden in einzelnen Einrichtungen für Praktika nicht in Betracht gezogen oder diesen werden nur unter- oder unbezahlte Praktikumsplätze angeboten. Dazu wäre auch eine höhere Förderung, um generell ein umfangreicheres Angebot an geförderten Praktikumsplätzen anbieten zu können, hilfreich. Eine weitere Anregung betrifft die Öffentlichkeitsarbeit und Vermarktung der Förderung sowohl im Bereich der Unternehmen als auch der Universitäten und Fachhochschulen, um Studentinnen im Vorfeld besser über dieses Förderangebot zu informieren. Die Programmlinie könnte generell auch stärker dazu genutzt werden, um die Kooperationen zwischen Unternehmen und tertiären Bildungseinrichtungen zu verstärken. 


\section{4 | FEMtech Karriere und FEMtech Karriere-Check für KMU}

Die in diesem Kapitel dargestellten Programmlinien zielen darauf ab, Chancengleichheit zu fördern und in der Praxis von forschungs- und technologieintensiven Unternehmen und Forschungseinrichtungen umzusetzen. FEMtech Karriere unterstützt diese Organisationen bei der Umsetzung umfangreicher Projekte in diesem Bereich, während der FEMtech Karriere-Check für KMU in den Jahren 2015 und 2016 für diese Unternehmensgruppe angeboten wurde, um sie bei der Durchführung einer Genderanalyse zu unterstützen und auf ein mögliches nachfolgendes FEMtech Karriere Projekt vorzubereiten.

\subsection{1 | FEMtech Karriere}

FEMtech Karriere unterstützt Unternehmen im Bereich der Forschung und Entwicklung sowie außeruniversitäre Forschungseinrichtungen in Naturwissenschaft und Technik bei der gezielten Förderung von Frauen und der Umsetzung von Maßnahmen für Chancengleichheit. Dabei können Projektaktivitäten zum Aufbau von Genderkompetenz im Unternehmen, zur Verbesserung des Personalmanagements (z. B. Recruiting, Employer Branding), zur Herstellung der Work-LifeBalance (u. a. auch Karenzmanagement), zur Förderung und Karriereentwicklung qualifizierter Mitarbeiterinnen und zur Neugestaltung der Öffentlichkeitsarbeit gesetzt werden.

Tabelle 4 | Eckpunkte zu FEMtech Karriere

\begin{tabular}{|c|c|}
\hline FEMtech Karriere & Kurzbeschreibung \\
\hline Ziele & $\begin{array}{l}\text { Die Schaffung fairer Rahmenbedingungen für Frauen und Männer in FTI- } \\
\text { Unternehmen sowie } \\
\text { die Erhöhung des Anteils von Frauen auf allen Hierarchieebenen sowie ihrer } \\
\text { Karrierechancen in den geförderten Unternehmen }\end{array}$ \\
\hline Zielgruppen & $\begin{array}{l}\text { Unternehmen (mit eigener Forschungs- und Entwicklungsabteilung oder mit } \\
\text { FTI-Projekten und mit Bedarf an hochqualifizierten Forscherinnen/Technike- } \\
\text { rinnen) und außeruniversitäre Forschungseinrichtungen mit naturwissen- } \\
\text { schaftlich-technischem Schwerpunkt (Fördernehmerlnnen) } \\
\text { Frauen und Männer in forschungs- und technologieintensiven Unternehmen } \\
\text { und außeruniversitären Forschungseinrichtungen im naturwissenschaftlich- } \\
\text { technischen Bereich }\end{array}$ \\
\hline Inhalt & $\begin{array}{l}\text { Förderung von Maßnahmen, die zur Chancengleichheit führen und Forsche- } \\
\text { rinnen/Technikerinnen in der Umsetzung ihrer beruflichen Ziele unterstüt- } \\
\text { zen. }\end{array}$ \\
\hline Einreichverfahren & $\begin{array}{l}\text { Laufende Einreichung mit Ausschreibungen in den Jahren 2011, 2012, } \\
2013,2014,2015,2016,2017,2018,2019\end{array}$ \\
\hline Laufzeit & Projektdauer: 6 bis 24 Monate \\
\hline Förderhöhe & $\begin{array}{l}\text { max. } € 50.000 \text { pro Projekt (Förderquote } 50 \%-70 \% \text { in Abhängigkeit von der } \\
\text { Unternehmensgröße) }\end{array}$ \\
\hline
\end{tabular}


Mit FEMtech Karriere wird generell versucht, faire Rahmenbedingungen für Frauen und Männer in Forschung, Technologie und Innovation in Unternehmen zu schaffen, die Karrierechancen von Frauen in den Unternehmen zu erhöhen und die Genderkompetenz in den Organisationen zu verbessern.

Bei den befragten Projektteilnehmerlnnen steht vor allem die Steigerung der Attraktivität der Unternehmen als ArbeitgeberInnen im Vordergrund. In diesem Zusammenhang wird laut Projektberichten teilweise auch die Positionierung als gendersensibles, unterstützendes Unternehmen angestrebt. Neben Aktivitäten im Bereich der Öffentlichkeitsarbeit sollen die Arbeitsbedingungen und die Möglichkeiten zur Work-Life-Balance weiter optimiert sowie die Außendarstellung des Unternehmens verbessert werden. Die Attraktivierung soll häufig zur verstärkten Ansprache von Frauen als potentielle Mitarbeiterinnen beitragen. Eine weitere wesentliche Zielsetzung der FEMtech Karriere Projekte ist der Aufbau und die Vertiefung der Genderkompetenz. Im Zuge der Projekte soll die Verstärkung der gendersensiblen Kommunikation und die aktive Verankerung von Genderaspekten erfolgen. Außerdem widmen sich die Projekte gezielt der langfristigen Karriereplanung und versuchen, nachhaltige Karrieremöglichkeiten für Frauen zu schaffen. Oftmals ist im Rahmen der Projektaktivitäten auch die gezielte Förderung von Qualifizierungsaktivitäten von Frauen vorgesehen, um sie auf ihrem Karriereweg zu unterstützen.

Grafik 37 | Hauptzielsetzungen der FEMtech Karriere Projekte, Anzahl der befragten Organisationen (Mehrfachnennungen möglich)

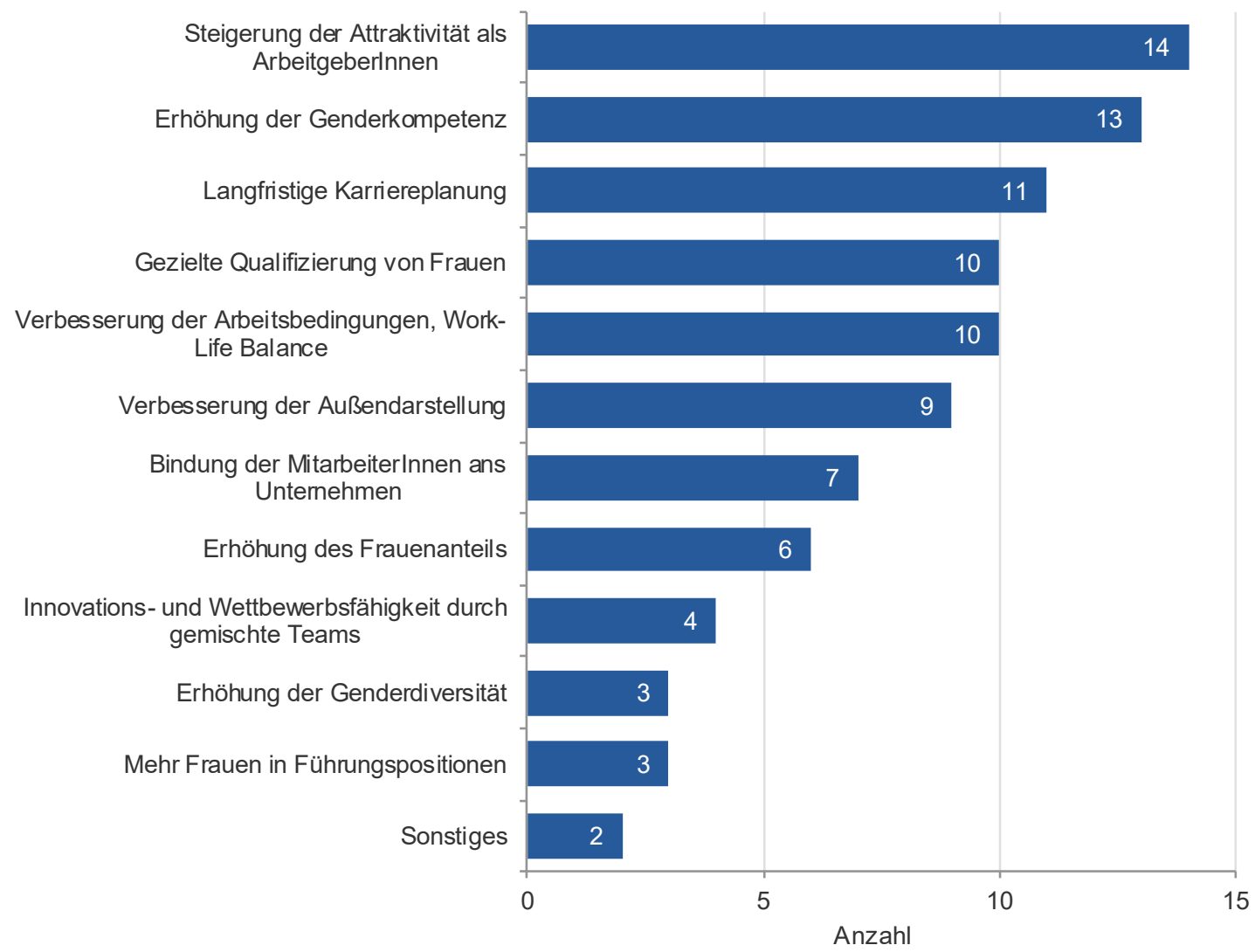

Quelle: FEMtech Karriere Befragung 2020, KMU Forschung Austria, n=18 


\section{Projektinhalte}

Die FEMtech Karriere Projekte setzen sich aus zwei Pflichtmodulen (Aufbau Genderkompetenz und Projektmanagement) und mehreren Wahlmodulen aus dem Bereich Chancengleichheit (Öffentlichkeitsarbeit, Personalmanagement, Work-Life-Balance und ein frei wählbares Modul) und dem Bereich Frauenförderung (mit den Wahlmodulen Coaching / Mentoring, Karriereentwicklung und ein frei wählbares Modul) zusammen. Um eine nachhaltige Einbettung des Projekts in bestehende Unternehmensstrukturen zu gewährleisten, wird ein vielfältiges Maßnahmenportfolio durch die Kombination mehrerer Wahlmodule empfohlen.

Das Pflichtmodul „Aufbau von Genderkompetenz“ sieht ein verpflichtendes Training der Führungskräfte und Schlüsselpersonen sowie eine Genderanalyse im Unternehmen vor. Dieses wird zumeist von externen GenderexpertInnen im Rahmen von Workshops und Coachings durchgeführt. Auch eine Analyse der Ausgangslage des Unternehmens in Bezug auf Gender ist vorgesehen, wozu beispielsweise eine Situationsanalyse anhand der Gender Score Card oder die 4RAnalyse zur Verteilung von Ressourcen, Rechten, Repräsentation und Realität zum Einsatz kommen kann. Zur nachhaltigen Forcierung der Genderagenden wird in einigen Unternehmen die Funktion einer Genderbeauftragten etabliert, Genderaktionspläne definiert, Gender und Diversity Leitfäden erstellt und Richtlinien für gendersensible Sprache erarbeitet.

Das zweite Pflichtmodul „Projektmanagement" stellt die effiziente Koordination und Abwicklung des FEMtech Karriere Projekts sicher. Neben der Planung und der Überwachung der Umsetzung der Maßnahmen muss auf die aktive Einbindung der Führungsebene und eine Geschlechterausgewogenheit im Projektteam geachtet werden.

Im Wahlmodul „Öffentlichkeitsarbeit" steht die Verankerung gendersensibler Kommunikation in den PR-Materialien im Vordergrund, um die Außenwirkung des Unternehmens zu verbessern. Die Unternehmen haben hierfür oftmals ihre Websites in Hinblick auf gendersensible Inhalte überarbeitet, bei ihrem Online-Auftritt weibliche Role Models und Erfolgsgeschichten präsentiert und Online-Bewerbungs- oder Karriere-Tools auf den Websites ergänzt. Auch Informationsmaterialien, Broschüren und Flyer wurden gendergerecht aufbereitet und Unternehmensleitbilder entsprechend angepasst bzw. eigene „Code of Conducts“ entworfen.

Im Rahmen des Wahlmoduls „Personalmanagement“ gilt es, das Unternehmen für potentielle Mitarbeiterinnen im Bereich FTI attraktiv zu machen. Die Maßnahmen sollen hierfür eine genderkompetente Personalwerbung sowie Aktivitäten zur längerfristigen Bindung hochqualifizierter Mitarbeiterinnen umfassen. Die Unternehmen führen daher häufig Analysen zur Optimierung ihrer Stellenanzeigen durch, erstellen genderneutrale Ausschreibungsunterlagen und Anforderungsprofile, entwickeln Recruiting-Leitfäden oder stellen Informationsmappen zur Einschulung neuer MitarbeiterInnen zusammen. Zur Optimierung der Rekrutierungsprozesse entwerfen die Unternehmen teilweise auch Online-Bewerbungstools und Programme zur Nachwuchsförderung oder Mentoring-Curricula für neue MitarbeiterInnen. Zur Erhöhung der Bindung der Angestellten werden Entwicklungsmöglichkeiten, Karrieremodelle und Bildungspläne mit den MitarbeiterInnen entworfen, wie beispielsweise eine Potentiallandkarte zur Karriereentwicklung oder persönliche Karrierebilder, Leitfäden für MitarbeiterInnen- und Karrieregespräche konzipiert sowie Coaching- 
und Mentoringangebote zur Karriereplanung umgesetzt. Um ein kontinuierliches Gendermonitoring der Situation der MitarbeiterInnen zu gewährleisten, entwirft ein Unternehmen beispielsweise ein eigenes Controllingtool für Genderkennzahlen.

Das Modul Work-Life-Balance hat zum Ziel, die individuelle Lebens- und Laufbahnplanung der MitarbeiterInnen mit den betrieblichen Anforderungen in Einklang zu bringen. Im Rahmen dieses Moduls setzen die Unternehmen in erster Linie Aktivitäten, um die Arbeitsbedingungen und -zeiten weiter zu verbessern und zu flexibilisieren, z. B. durch Verstärkung von Home-Office-Möglichkeiten oder die Einführung einer 4-Tage Woche. Auch organisatorische Vorbereitungen für Karenzen / Auszeiten werden getroffen sowie im Anschluss daran Wiedereinstiegs- und Coachingprogramme implementiert. Die vielfältigen Aktivitäten zur Förderung der Work-Life-Balance werden teilweise in eigenen Aktionsplänen und Leitfäden festgehalten.

Im Modul "Coaching/Mentoring“ werden Forscherinnen und Technikerinnen bei ihrer Karriereentwicklung gefördert. Mit dem Einsatz von Coaching / Mentoringangeboten im Bereich Führung und Management wird versucht, Frauen verstärkt in Führungspositionen zu bringen. Die identifizierten MitarbeiterInnen werden in ihrer persönlichen Entwicklung und bei der Übernahme neuer Leitungspositionen gezielt unterstützt. Da das Coaching oft in einem direkten Zusammenhang mit einem Karrieresprung steht, werden die Module „Coaching / Mentoring“ und „Karriereentwicklung" zumeist in Kombination umgesetzt.

Die Schwerpunkte des Moduls „Karriereentwicklung“ sind, die Führungskompetenzen von Forscherinnen und Technikerinnen zu stärken und die Leistungen von Frauen im Unternehmen besser sichtbar zu machen. Dazu entwickeln die Unternehmen gezielte Qualifizierungs- und Trainingsprogramme für Frauen, setzen Einzelcoachings und Workshops zur Karriereentwicklung um oder etablieren Mentoring-Programme als verbindliche Personalentwicklungsmaßnahmen. Um die Transparenz im Unternehmen zu fördern, werden beispielsweise auch firmeninterne Organigramme um eine Beschreibung der Positionen und Gehaltsstufen erweitert, die Rollen bestimmter Leitungsfunktionen genauer definiert und die Diversität von Teams gezielt gefördert. Auch Vernetzungsinstrumente, wie ein Journal Club für Nachwuchsforschende, werden etabliert, um den Austausch von ForscherInnen zu forcieren.

Zusätzlich können die Unternehmen / Organisationen sowohl im Bereich von Chancengleichheit als auch im Bereich der Frauenförderung ein frei wählbares Wahlmodul definieren, das zu den Zielen von FEMtech Karriere passt und auf die Ausgangslage des Unternehmens abgestimmt ist. Einige wenige Unternehmen machen von dieser Möglichkeit Gebrauch und führen beispielsweise eine Detailanalyse ihrer Organisation durch und entwickeln Maßnahmen zur Institutionalisierung von Gender Mainstreaming. Auch eine gendergerechte Software wird entwickelt und Monitoringinstrumente konzipiert, um Fortschritte im Bereich der Chancengleichheit zu beobachten. Einzelne Unternehmen widmen sich dem Wissensmanagement und dem Know-how-Aufbau oder arbeiten Weiterbildungskonzepte unter Berücksichtigung von Genderaspekten aus. 


\subsection{2 | FEMtech Karriere-Check für KMU}

Im Jahr 2015 und 2016 wurde der FEMtech Karriere-Check für KMU ins Leben gerufen, um die Chancengleichheit von Frauen und Männern in FTI-intensiven KMU im naturwissenschaftlichtechnischen Bereich zu unterstützen. Dieser bot KMU eine einfache Möglichkeit, in das Thema Chancengleichheit einzusteigen und die Situation im eigenen Unternehmen in Hinblick auf Chancengerechtigkeit zu analysieren. Dieser sollte auch gleichzeitig als Vorbereitung für zukünftige FEMtech Karriere Projekte dienen.

Tabelle 5 | Eckpunkte zu FEMtech Karriere-Check für KMU

\begin{tabular}{|c|c|}
\hline $\begin{array}{l}\text { FEMtech Karriere-Check } \\
\text { für KMU }\end{array}$ & Kurzbeschreibung \\
\hline Ziele & $\begin{array}{l}\text { Förderung der Chancengleichheit von Frauen und Männern in forschungs- } \\
\text { und technologieintensiven KMU im naturwissenschaftlich-technischen Be- } \\
\text { reich }\end{array}$ \\
\hline Zielgruppen & $\begin{array}{l}\text { Kleine und mittlere Unternehmen mit eigener Forschungs- und Entwick- } \\
\text { lungsabteilung bzw. mit FTI-Projekten oder mit Bedarf an hochqualifizierten } \\
\text { Forscherinnen / Technikerinnen, mit naturwissenschaftlich-technischem } \\
\text { Schwerpunkt und Standort in Österreich }\end{array}$ \\
\hline Inhalt & $\begin{array}{l}\text { Mit dem FEMtech Karriere-Check erhalten kleine und mittlere Unternehmen } \\
\text { Unterstützung, um ihr Unternehmen in Hinblick auf Chancengerechtigkeit zu } \\
\text { analysieren. Dabei wird mit Hilfe von externem Know-how eine Genderana- } \\
\text { lyse erstellt und darauf aufbauend Maßnahmen zur Gleichstellung von } \\
\text { Frauen und Männern abgeleitet. }\end{array}$ \\
\hline Einreichverfahren & Laufende Einreichung bis zur Mittelausschöpfung in den Jahren 2015, 2016 \\
\hline Laufzeit & Projektdauer: max. 12 Monate \\
\hline Förderhöhe & max. 10.000,- (mit einer Förderungsquote von $80 \%$ ) \\
\hline
\end{tabular}

Der FEMtech Karriere-Check wurde im Jahr 2015 von 6 Unternehmen und im Jahr 2016 von 11 Unternehmen genutzt. Insgesamt kamen daher 17 geförderte Genderanalysen mit Hilfe des FEMtech Karriere-Checks zustande. Lediglich 5 Unternehmen (29\%) setzten ausschließlich den FEMtech Karriere-Check um. Die Mehrheit der Unternehmen (12 bzw. $71 \%$ ) baute auf den Genderanalysen und dabei abgeleiteten Maßnahmenempfehlungen auf und reichte im Anschluss ein FEMtech Karriere Projekt erfolgreich ein. D. h. gerade für KMU war das Angebot des FEMtech Karriere-Checks als Einstieg hilfreich, um sich mit dem Thema Gender Mainstreaming und Chancengleichheit (intensiver) befassen zu können. Erst darauf aufbauend konnten umfangreichere Projekte in diesem Themenbereich realisiert werden. 


\subsection{3 | Projektanalyse FEMtech Karriere und FEMtech Karriere-Check für KMU}

Im Zeitraum von 2011 bis 2019 wurden insgesamt 81 FEMtech Karriere und FEMtech KarriereChecks für KMU eingereicht. Insgesamt erteilte die FFG im Analysezeitraum 52 Projekten die Genehmigung zur Durchführung (35 FEMtech Karriere Projekte und 17 FEMtech KarriereChecks für KMU), ein FEMtech Karriere Projekt musste jedoch aus wirtschaftlichen Gründen abgebrochen werden. In den Jahren 2017 und 2019 wurden am meisten FEMtech Karriere Projekte eingereicht.

Grafik 38 | Entwicklung der Anzahl der FEMtech Karriere und der FEMtech Karriere-Check Projekte, Ausschreibungen 2011-2019

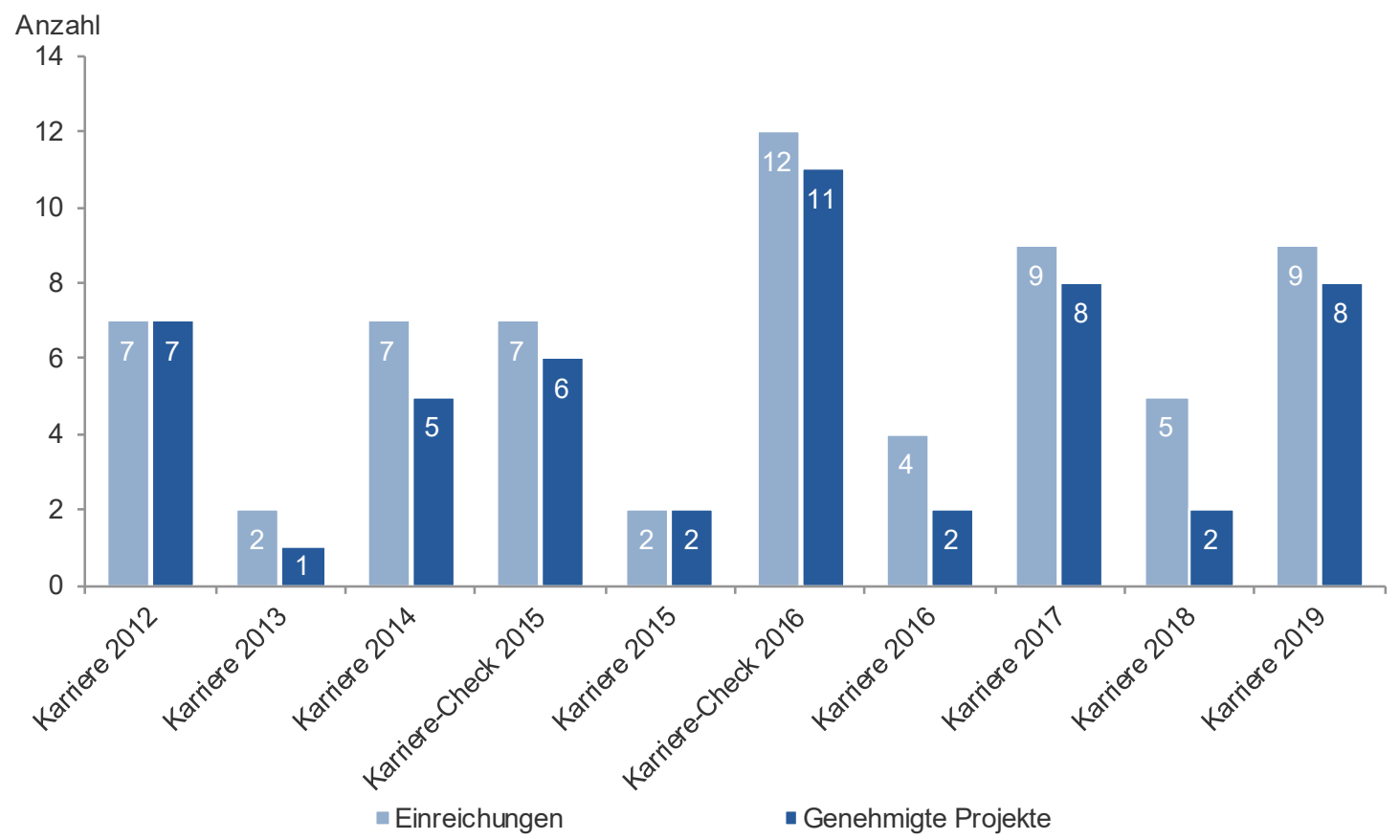

Quelle: FFG; Berechnung und Darstellung KMU Forschung Austria

Die 51 FEMtech Karriere und FEMtech Karriere-Check Projekte wurden in 35 verschiedenen Unternehmen bzw. Organisationen implementiert. 15 Organisationen setzten dabei FEMtech Karriere-Check und FEMtech Karriere Projekte bzw. mehrere FEMtech Karriere Projekte um. 15 Organisationen realisierten jeweils 1 FEMtech Karriere Projekt, wie Grafik 39 zeigt.

An den FEMtech Karriere Projekten (inklusive des FEMtech Karriere-Checks) beteiligten sich in erster Linie Unternehmen. Diese sind vorwiegend im Bereich der Informationsdienstleistungen, der Metallerzeugung und dem Maschinenbau sowie im Bau-/Ingenieur- und Architekturwesen tätig. Mehr als drei Viertel der Unternehmen sind KMU, da der Karriere-Check gezielt auf diese Zielgruppe ausgerichtet war. Die 5 involvierten Großunternehmen setzen nur FEMtech Karriere Projekte um. Neben Unternehmen werden die FEMtech Karriere Projekte auch in Kompetenzzentren, außeruniversitären Einrichtungen und kooperativen Forschungseinrichtungen realisiert. 


\section{Projektarten der Organisationen}

mehrere FEMtech Karriere Projekte

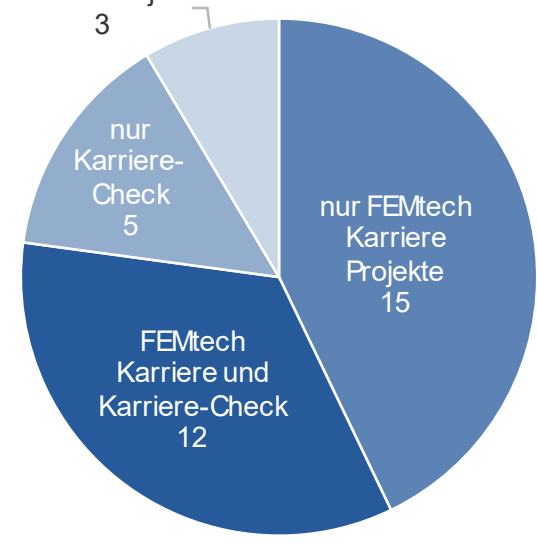

\section{Beteiligte Organisationen}

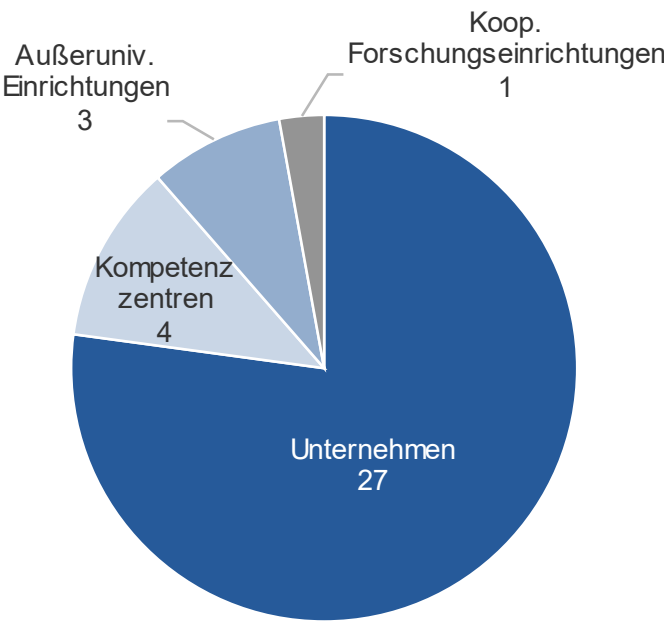

Quelle: Projektdatenbank der FFG (2011-2019), n=35

Die verschiedenen Unternehmen und Organisationen wurden in erster Linie durch die direkte Ansprache durch die FFG im Rahmen von Newslettern, Veranstaltungen oder Messen sowie durch Online-Medien, Social Media und Internetrecherchen auf die Programmlinien FEMtech Karriere und FEMtech Karriere-Check für KMU aufmerksam, wie die befragten ProjektteilnehmerInnen angeben. Der FFG und deren Online-Angebot kommt eine wichtige Bedeutung bei der Streuung der Ausschreibungsinformationen zu. Auch bei den Fallstudien zeigt sich, dass vor allem die aktive Suche auf der FFG-Homepage ausschlaggebend war, um von der Ausschreibung zu erfahren.

Wir durchforsten immer die FFG Ausschreibungen und sind darauf gestoßen.

Wir haben bereits Praktikantinnen gehabt und waren deshalb öfter auf der FFG-Homepage und haben so die Ausschreibung entdeckt.

Die Initiative und Entscheidung zur Teilnahme wird zumeist von der Geschäftsführung gefällt, wie 12 von 20 befragten Organisationen bekannt geben. Die Beteiligung an FEMtech Karriere bzw. dem FEMtech Karriere-Check für KMU wird somit mehrheitlich "top-down“ auf Ebene der Geschäftsführung entschieden. Dennoch geht die tatsächliche Initiative häufig von den MitarbeiterInnen aus, die nach den Ausschreibungen recherchieren, Projektideen konzipieren und für die Geschäftsführung vorbereiten. Die involvierten GeschäftsführerInnen stehen der gezielten Förderung von Frauen und der Unterstützung von Chancengleichheit sehr wohlwollend und forcierend gegenüber, was sich auch bei der Analyse der Fallstudien beobachten lässt. 
Grafik 40 | Aufmerksamkeit auf die Programmlinie und Initiative zur Teilnahme, Anzahl der befragten Organisationen

Aufmerksamkeit durch

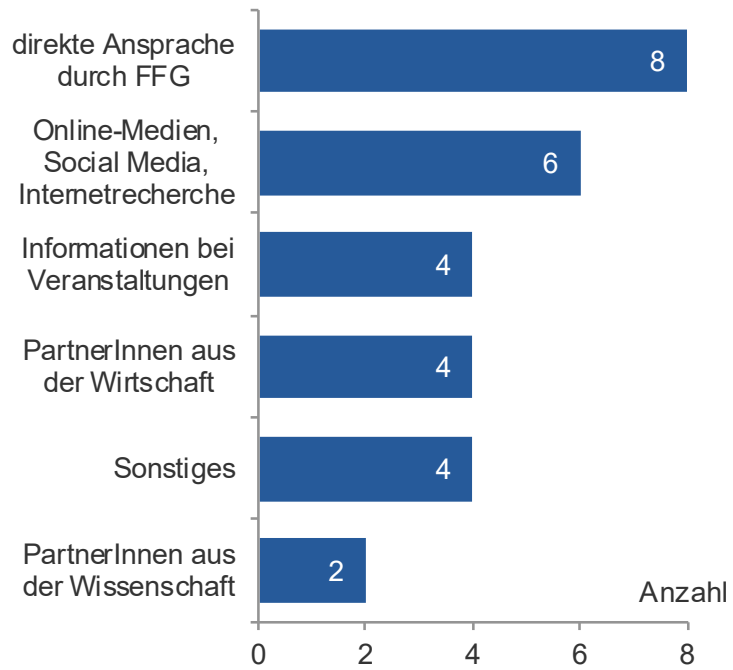

Initiative zur Teilnahme

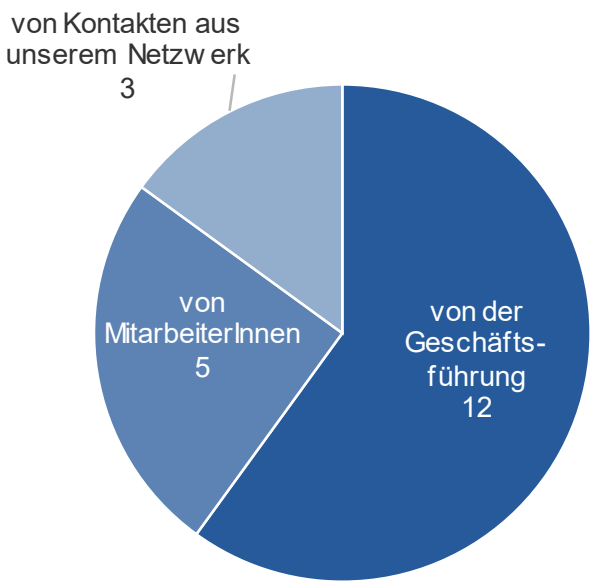

Quelle: FEMtech Karriere Befragung 2020, KMU Forschung Austria, n=20

Die Förderleistung trägt maßgeblich dazu bei, Aktivitäten im Bereich der Chancengerechtigkeit und Genderkompetenz umzusetzen. Ohne diese Förderung hätte mehr als die Hälfte der befragten Organisationen, die FEMtech Karriere Projekte umgesetzt haben, nur in deutlich reduziertem Maße die geplanten Aktivitäten realisieren können (siehe Grafik 41). Den meisten Organisationen fehlen sonst die finanziellen Ressourcen für eine ausführliche Auseinandersetzung mit diesen Themen. Mehr als ein Drittel (7 von 19) hätte ohne finanzielle Unterstützung von dem konzipierten Maßnahmenportfolio gänzlich Abstand nehmen müssen. Auch in den Fallstudien wird auf die Wichtigkeit der Förderung hingewiesen:

Ohne Förderung wäre es nicht in diesem Umfang möglich gewesen.

So flächendeckend über einen Zeitraum stark daran zu arbeiten wäre sonst niemals umsetzbar gewesen.

Auch die Durchführung des FEMtech Karriere-Checks für KMU wäre ohne das Förderangebot mehrheitlich gar nicht oder nur stark eingeschränkt möglich gewesen. Der Check wird als hilfreicher und notwendiger Einstieg in das Thema gesehen. Einige Organisationen mussten sich noch mehr Zeit für die Auseinandersetzung mit dem Thema nehmen und erst Wissen in diesem Bereich aufbauen, wie auch folgende Antworten zeigen.

Weil wir uns viel Zeit für die Auseinandersetzung mit dem Thema nehmen mussten und auch noch viel Wissen aufbauen mussten. Dies wäre ohne Projekt in der Form nicht möglich gewesen. 
Der Check war notwendig zum Einstieg, das Karriere-Projekt wäre als Anfangsprojekt zu groß gewesen.

Dennoch hätten 4 von 6 Organisationen, die auch den FEMtech Karriere-Check für KMU durchgeführt haben, versucht, auch ohne diese Förderung ein FEMtech Karriere Projekt zu realisieren.

Grafik 41 | Durchführung der FEMtech Karriere Projekte und des FEMtech Karriere-Checks für KMU ohne Förderung, Anzahl der befragten Organisationen

FEMtech Karriere Projekt ohne Förderung

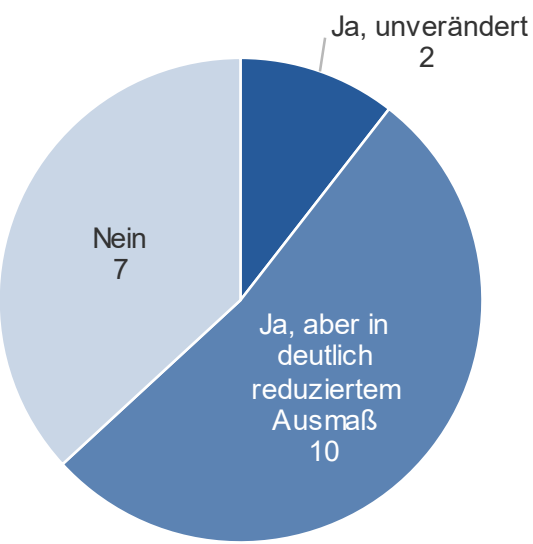

\section{FEMtech Karriere-Check für KMU ohne Förderung}

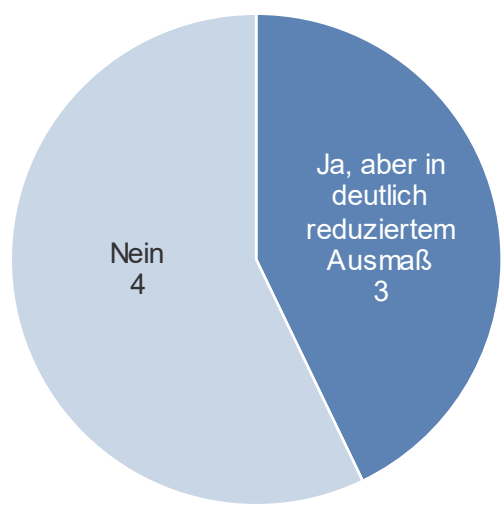

Quelle: FEMtech Karriere Befragung 2020, KMU Forschung Austria, n=19 (FEMtech Karriere) und n=7 (FEMtech Karriere-Check für KMU)

Auf die Umsetzung der Projektaktivitäten wirkt sich vor allem das vorherrschende Tagesgeschäft negativ aus. Auch die verfügbaren zeitlichen Ressourcen der Projektleiterlnnen und Mitarbeiterlnnen führen zu Herausforderungen bei der Abwicklung der Maßnahmen. Personalwechsel, Austritte und Karenzen erweisen sich bei einem Teil der Projekte als hinderlich für den weiteren Projektfortschritt. Mit Widerstand oder mangelndem Commitment von Seiten des Personals oder der Führungsebenen haben hingegen nur eine Minderheit der befragten ProjektteilnehmerInnen zu kämpfen.

Auch die Fallstudien zeigen, dass es im Geschäftsalltag oftmals nicht leicht ist, sich die Zeit für die Projektaktivitäten zu nehmen. Dennoch wäre es ohne das Förderangebot noch wesentlich schwieriger, sich Gender- und Gleichstellungsthemen zu widmen. Der Projektrahmen schafft für viele erst die Möglichkeit, sich damit intensiver auseinanderzusetzen.

Auch wenn man jetzt den Projektrahmen hat, muss man sich dafür die Zeit freischaufeln. Aufträge nehmen darauf keine Rücksicht und man kann diese deswegen auch nicht ablehnen. 
Die Hauptschwierigkeit war, alle an einem Termin zusammenzubringen. Also, es waren Leute in Karenz, die aber doch versucht haben, daran teilzunehmen. Das war die Herausforderung. Auch in Abstimmung mit der Arbeitsauslastung natürlich.

Das Genderthema läuft immer ein bisschen mit. KMU haben meist keine Zeit dafür. Vorteil der Förderung ist, dass man sich extra dafür Zeit nimmt dank der Förderung. Das hat man nur über so ein Projekt.

Grafik 42 | Herausforderungen bei der Umsetzung der FEMtech Karriere Projekte bzw. des FEMtech Karriere-Checks für KMU, Anzahl der befragten Organisationen

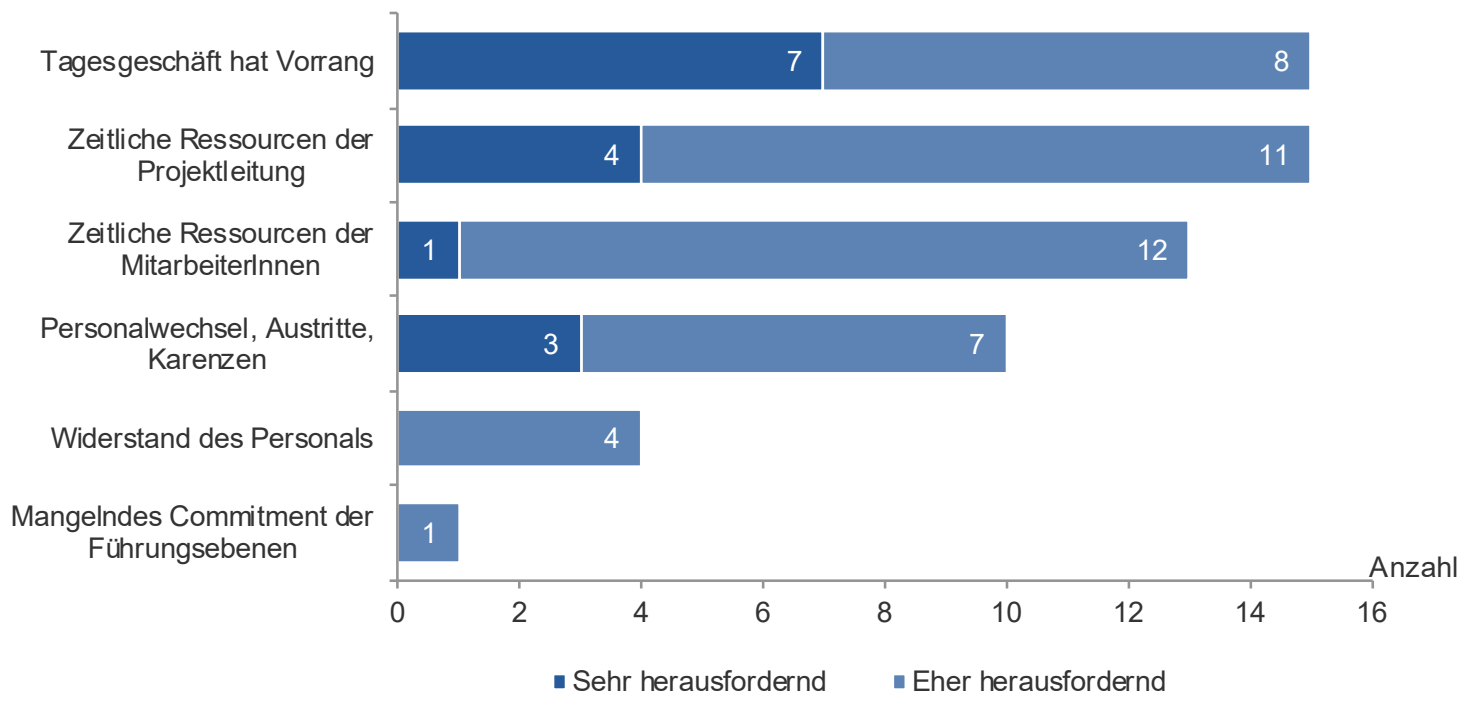

Quelle: FEMtech Karriere Befragung 2020, KMU Forschung Austria, n=18

\subsection{4 | Ergebnisse und Wirkungen sowie Beurteilung der Programmlinien}

\section{Ergebnisse und Wirkungen des FEMtech Karriere-Checks für KMU}

Die Zielsetzung des FEMtech Karriere-Checks war, Genderkompetenz in den KMU aufzubauen, Genderanalysen durchzuführen und darauf aufbauend Maßnahmenvorschläge zur Gleichstellung von Frauen und Männern zu entwickeln. Mit der Durchführung dieser Aktivitäten wurden in erster Linie GenderexpertInnen, Consultants und UnternehmensberaterInnen betraut. Diese führten Analysen der Unternehmensdaten und -dokumente (z. B. Frauenanteile, Verwendung gendersensibler Sprache), Befragungen und Interviews des Personals und der Führungskräfte, Workshops zur Sensibilisierung und Ideensammlung sowie Prozess- und Produktanalysen durch.

Auf Basis dieser umfassenden Erhebungen wurden Maßnahmenvorschläge zur Verbesserung der Gendergerechtigkeit abgeleitet. Die Maßnahmenpläne umfassten folgende Themenbereiche:

- Aufbau von Genderwissen und Nominierung von Genderbeauftragten, Erarbeitung von Gleichstellungsrichtlinien 
- Öffentlichkeitsarbeit: Erhöhung der Attraktivität der Unternehmen als ArbeitgeberInnen und der Sichtbarkeit von Frauen im Unternehmen (z. B. auf der Homepage)

- Geschlechtergerechte Sprache bei Unternehmensdokumenten

- Entwicklung von gendergerechten Unternehmensleitbildern

- Optimierung der Rekrutierungsprozesse (der Stellenbeschreibungen und der Auswahlverfahren)

- Karriereentwicklung und MitarbeiterInnengespräche

- Qualifizierung, Weiterbildung und Trainings für Frauen

- Work-Life-Balance, Karenz und Wiedereinstieg

Der Großteil der Unternehmen (71 \% bzw. 12 von 17) nutzte diese Maßnahmenempfehlungen, um darauf aufbauend ein FEMtech Karriere Projekt zu realisieren.

Auch die Fallstudien zeigen, dass der FEMtech Karriere-Check für KMU den Unternehmen einen guten Einstieg in die Thematik bot. Einige waren mit der Antragstellung für ein FEMtech Karriere Projekt noch überfordert, so dass vorab noch Wissen und Know-how in diesem Bereich aufgebaut werden musste.

Es war insofern eine Hilfe, weil es für die Bewusstseinsbildung wichtig war. Dass man sich gewisse Themen wirklich anschaut, die Zahlen analysiert und intern über Gleichberechtigung spricht. Auch die Kommunikationsprozesse wurden analysiert, wo man noch achtsamer werden könnte. Es hat auf jeden Fall viel gebracht, sich damit auseinander zu setzen und das Thema auch immer wieder im Alltag in Erinnerung zu rufen.

Wir haben sehr wichtige Grundlagen erarbeitet, die wir auch heute noch für die tägliche Arbeit brauchen.

\section{Ergebnisse und Wirkungen der FEMtech Karriere Projekte}

Entsprechend der Zielsetzungen der FEMtech Karriere Projekte wurde laut befragten ProjektteilnehmerInnen vor allem eine Erhöhung der Genderkompetenz (siehe Grafik 43) erreicht. Das Pflichtmodul „Aufbau von Genderkompetenz“ hat geholfen, die Thematik den MitarbeiterInnen aller Hierarchieebenen näher zu bringen und als Querschnittsmaterie in den Unternehmen zu verankern. Die vielfältigen Sensibilisierungs- und Schulungsaktivitäten haben zu einem Aufbau eines Gender-Bewusstseins geführt und das Verständnis von Geschlechtergerechtigkeit in den Unternehmen verbessert. Auch die vielfältigen entwickelten Produkte, wie Gender-Leitfäden oder Genderaktionspläne, helfen, sich weiterhin Genderanliegen zu widmen und das Engagement in diesem Bereich fortzusetzen.

Auch die Fallstudien zeigen, dass die Aktivitäten zur Erhöhung der Genderkompetenz vor allem zu Bewusstseinsveränderung in den Organisationen und zu einer verstärkten Sensibilität für diese Themen beigetragen haben. Vor allem die Einbindung der Führungskräfte und aller MitarbeiterInnen in die Projektaktivitäten wird als wesentlich erachtet und hat zu Veränderungen in der täglichen Arbeit, z. B. bei der Erstellung von Projektanträgen, geführt. 
Die Möglichkeit mit den Führungskräften in Workshops zu arbeiten, sie wirklich anzusprechen und auf das Thema aufmerksam zu machen, war wichtig.

Das gemeinschaftliche Arbeiten an etwas sehr Essenziellem, was eigentlich regelmäßig passieren sollte und zu sehen, wie die Kollegen immer mehr mit dem Thema anfangen können, waren die Highlights.

Mir kommt schon vor, dass sich im Bewusstsein der Mitarbeiter einiges getan hat. Gerade bei Projektanträgen, wo man die Frage der Gleichstellung und der Gender-Diversität beantworten muss, gibt es jetzt doch einige, die aktiv auf uns zu kommen und nachfragen, wie man das am besten machen kann.

Insbesondere die Nominierung von Genderbeauftragten stellt die nachhaltige Berücksichtigung der Genderthematik sicher. In 11 von 17 befragten Organisationen wurde seit der Durchführung des FEMtech Karriere-Checks für KMU bzw. der FEMtech Karriere Projekte eine Gendervertrauensperson oder ein/e Gleichstellungsbeaufte/r langfristig installiert. Die Vertrauenspersonen konnten mit Hilfe der Projekte ihr Wissen vertiefen und ihre Position festigen, wie sich bei den Fallstudien beobachten lässt.

Positiver Effekt des Projekts ist, dass sich meine Position als Gleichstellungsbeauftragte festigen konnte. Ich bin im Unternehmen über diese Projekte gut als solche angekommen.

Das Genderthema hat an Präsenz gewonnen und ich fühle mich in meiner Position als Gleichstellungsbeauftragte wohler, weil ich die Möglichkeit gehabt habe, dazu Kompetenzen aufzubauen. Je mehr ich mich damit beschäftige, desto wichtiger wird es für mich auch. Ich merke, wo es überall Handlungsbedarf gibt.

Auch im Bereich der Außendarstellung und der Steigerung der Attraktivität als Arbeitgeberln wurden die gewünschten Ziele erfüllt, wenngleich hierbei noch deutlich weiterer Optimierungsbedarf gesehen wird. Zur Verbesserung der Außenwirkung wurden zahlreiche Aktivitäten im Bereich der Öffentlichkeitsarbeit gesetzt. Neben der gendergerechten Überarbeitung der Websites und der Attraktivierung der Online-Auftritte durch Präsentation weiblicher Role Models wurden auch diverse Unternehmensbroschüren und Flyer (wie z. B. auch Kompetenz-Visitenkarten) gendersensibel überarbeitet und verteilt. Aktive Medienaussendungen, via Newsletter, Online-Postings etc, wurden verstärkt, Veranstaltungsbesuche und Vorträge von Forscherinnen forciert und Kooperationen zu Schulen und Hochschulen ausgebaut. Auch die erfolgreiche Teilnahme an Wettbewerben oder die Darstellung als Good Practice in den Medien verbesserte das Image der Unternehmen als gendersensible und familienfreundliche ArbeitgeberInnen, wie auch folgender Bericht aus einer Fallstudie zeigt.

Bei uns in der Steiermark gibt es jedes zweite Jahr den Wertgeschätzt Wettbewerb, wo Unternehmen einreichen können. Da waren wir im Frühjahr auch in der Endrunde, wir haben zwar nicht gewonnen, aber wir haben uns gefreut, dass wir dort sichtbar waren. Es wurde in den Medien durchaus über den Bewerb berichtet. 
In Bezug auf die Verbesserung der Arbeitsbedingungen und der Work-Life-Balance liegt ebenfalls eine Zielerreichung vor, jedoch zum Teil mit dem Bedarf zur weiteren Optimierung der arbeitsbezogenen Rahmenbedingungen. Die Aktivitäten im Bereich der Work-Life-Balance wurden gesetzt, um sowohl die Bindung bestehender Mitarbeiterlnnen zu steigern als auch neue Personenkreise anzusprechen. Dazu wurden die Arbeitsbedingungen verbessert und die Arbeitszeiten weiter flexibilisiert, wie z. B. durch die Implementierung einer 4-Tage-Woche oder von Home-Office Angeboten. Um für Karenzen oder Auszeiten besser vorbreitet zu sein, wurden organisatorische Vorkehrungen getroffen und Wiedereinstiegsprogramme implementiert. Die dafür entworfenen Aktionspläne, Programme und Leitfäden unterstützen die Unternehmen dabei, sich auch in Zukunft nachhaltig diesem Thema zu widmen, wie auch die Fallstudien zeigen.

Nachhaltig sind die Prozesse, die wir genauer definieren und eingeführt haben und dass flexibles Arbeiten viel normaler und bei der Geschäftsführung auch akzeptierter geworden ist.

Das Zusammenleben mit der Familie wird bei uns sehr gefördert, die Work-Life-Balance. Es kommt auch dazu, dass unsere Tätigkeit, die Forschungsarbeit, natürlich auch eine gewisse Flexibilität ermöglicht.

In Hinblick auf die gezielte Qualifizierung von Frauen und die langfristige Karriereplanung weiblicher MitarbeiterInnen konnten die angestrebten Zielsetzungen mehrheitlich erreicht werden. Insbesondere in Bezug auf die langfristige Karriereplanung wird angemerkt, dass diese allerdings zum Teil noch nicht in dem gewünschten Ausmaß umgesetzt werden konnte. Um Forscherinnen und Technikerinnen gezielt bei ihrer Karriereentwicklung zu fördern und Frauen verstärkt in Führungspositionen zu bringen, kamen zahlreiche Coaching-/Mentoring-Angebote sowie vielfältige Maßnahmen zur Förderung der Karriereentwicklung, wie beispielsweise Schulungsund Karriereentwicklungsprogramme für Nachwuchskräfte, oder ein Karrieremanagement mit laufenden Karriere-/Mitarbeiterlnnengesprächen zum Einsatz. Aufgrund dieser Bemühungen konnten Mitarbeiterinnen für Projektmanagementaufgaben sowie Teamleiterinnen gewonnen werden. Das Selbstbewusstsein der Mitarbeiterinnen wurde gestärkt und sie haben individuelle Karrierepfade für sich entworfen. Die Vielfalt der Aktivitäten zur Unterstützung der Karriereplanung und der hohe persönliche Nutzen wird auch bei den Fallstudien deutlich.

Das Karrieremodell mit dem transparenten Gehaltsschema ist bei den Mitarbeiterlnnen sehr gut angekommen. Man kann jetzt sehr gut nachvollziehen, warum jemand wo eingestuft ist und wie viel jemand in dieser Stufe bekommt.

Es wurden auch die Verantwortungsbereiche neu verteilt. Wir sind in Teams organisiert, wir haben eine sehr flache Hierarchie. Den Teamleitern ist mehr Verantwortung zugekommen und den einzelnen Mitarbeitern. Jeder einzelne hat mehr Verantwortung bekommen, die er zu tragen hat. Das ist wirklich bleibend.

Mir persönlich hat der Führungskräfte-Workshop sehr viel gebracht. Das war wirklich ein tolles Programm, weil wir Kommunikationstechniken erlernt haben. Das verwende ich heute noch.

Das Beste war wirklich, dass der Coach gekommen ist. Das Coaching hat geholfen, Dinge an sich selbst zu verbessern. Man konnte sich individuell „briefen“ Iassen. 
Gleichzeitig wurden auch soziale Aktivitäten ins Leben gerufen. Dadurch entstand eine höhere Teamidentität und die Zusammenarbeit der MitarbeiterInnen verbesserte sich. Dies lässt sich auch bei den Fallstudien beobachten.

In Summe, dass sich das Teamverständnis und der Teamspirit erhöht hat über dieses Thema. Da nur Frauen daran teilnehmen durften, hat es das Frauen-Team extrem zusammengeschweißt. Das war für die Frauen-Power echt super.

Die einzelnen Mitarbeiter fühlen sich alle mehr wertgeschätzt. Auch untereinander fühlen sie sich mehr als Teil eines Ganzen. Auch das Bild nach außen wurde klarer.

Es hat das Team viel besser zusammengeschweißt. Es sind auch neue Forschungsprojekte daraus entstanden, weil die Leute miteinander geredet haben.

Zur Steigerung des Frauenanteils in Unternehmen, bei Führungspositionen und zur Erhöhung der Genderdiversität in den Organisationen wird noch weiteres Optimierungspotential gesehen, da diese Zielsetzungen zum Teil noch nicht im gewünschten Ausmaß erreicht werden konnten, wenngleich diese Zielbereiche keine hohe Priorität bei den befragten Organisationen einnahmen. Zur Steigerung der Frauenanteile wurden vor allem Aktivitäten im Recruiting-Bereich gesetzt, wie beispielsweise Stellenanzeigen optimiert, gendersensible Leitfäden erstellt, Bewerbungstools konzipiert und Informationsmaterialien für neue MitarbeiterInnen zusammengestellt. Auch Kooperationen mit Schulen und Hochschulen und Veranstaltungen, wie der Girls Day, oder neu konzipierte PraktikanntInnenprogramme wurden genutzt, um Kontakte zu potentiellen weiblichen Arbeitskräften herzustellen. Durch gendersensible Optimierung der Recruitingprozesse sollten auch hochqualifizierte weibliche Arbeitskräfte verstärkt angesprochen und als neue Mitarbeiterinnen gewonnen werden.

Auch im Rahmen der Fallstudien haben die Aktivitäten im Recruiting-Bereich maßgeblich zur Bewusstseinsbildung und Verhaltensänderung, wie beispielsweise zur Optimierung der Stellenanzeigen beigetragen, die nun verstärkt auch Frauen ansprechen.

Ich habe immer geglaubt, wir machen die Ausschreibungen neutral, bis die Gleichstellungsbeauftragte darauf hingewiesen hat, was man anders machen könnte und was eher Frauen ansprechen würde. Dinge, an die ich selbst nie gedacht hätte. Und genau dafür muss sich jemand mit dem Thema beschäftigen. 
Grafik 43 | Erzielung folgender Wirkungen durch die FEMtech Karriere Projekte, Anzahl der befragten Organisationen

Erhöhung der Genderkompetenz

Verbesserung der Außendarstellung

Steigerung der Attraktivität als Arbeitgeberlnnen

Gezielte Qualifizierung von Frauen

Verbesserung der Arbeitsbed., Work-Life Balance

Langfristige Karriereplanung

Bindung der MitarbeiterInnen ans Untemehmen

Erhöhung des Frauenanteils

Mehr Frauen in Führungspositionen

Erhöhung der Genderdiversität

Innovations-/Wettbewerbsfähigkeit durch gemischte Teams

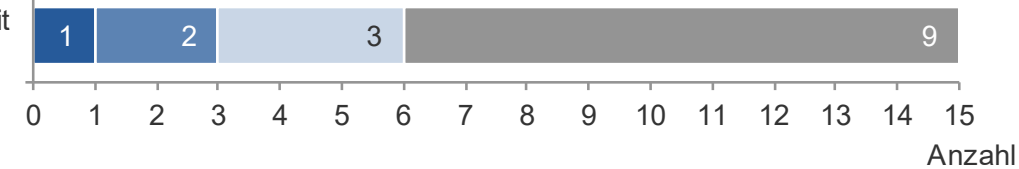

- umfassend erzielt

noch nicht im gewünschten Ausmaß erzielt
- erzielt, aber noch Optimierungsbedarf

- nicht relevant

Quelle: FEMtech Karriere Befragung 2020, KMU Forschung Austria, n=15

Konkret nach den Beschäftigungsdaten zu Projektbeginn im Vergleich zum Jahr 2020 gefragt zeigt sich, dass trotz vielfältiger Bemühungen, den Frauen- und Forscherinnenanteil in den Unternehmen zu erhöhen, dieser bei 6 von 10 befragten Organisationen gleichgeblieben ist. 4 von 10 beteiligte Unternehmen konnten Steigerungen der Frauenanteile verzeichnen. Diese betrafen in erster Linie den wissenschaftlich / technischen Bereich, aber in geringem Ausmaß auch Leitungs- und Führungspositionen.

Auch bei den Fallstudien war zu beobachten, dass die konkrete Einstellung von Frauen im technischen Bereich aufgrund des mangelnden Bedarfs in den Unternehmen und des begrenzten Angebots an Technikerinnen schwierig war.

Es wäre natürlich schön gewesen, wenn wir noch mehr Frauen hätten ansprechen können. Wir haben aber auch nicht so viele neue Personen eingestellt. 
Mein Eindruck war, man hat auch schon vor dem Projekt versucht, mehr Frauen zu finden. Es ist aber schwer Frauen in der Informatik zu finden, weil es wenige gibt in diesem Bereich.

Ein Teil der Projektteilnehmerlnnen kann auch auf eine steigende Zahl an weiblichen Bewerberinnen zurückgreifen. 7 von 16 befragten Organisationen haben den Eindruck, dass seit der Umsetzung des FEMtech Karriere Projekts bzw. des FEMtech Karriere-Checks für KMU mehr Bewerbungen von Frauen vorliegen. 9 Organisationen sind der Meinung, dass sich gleich viele Frauen wie vorher bewerben.

Auch hinsichtlich der Akzeptanz bzw. der Awareness für das Thema Gleichstellung hat sich in den beteiligten Unternehmen aufgrund der FEMtech Karriere Projekte bzw. des FEMtech Karriere-Checks für KMU einiges getan. 16 von 17 Befragten nehmen eine erhöhte Akzeptanz und Awareness für das Thema wahr, die Hälfte hat sogar den Eindruck, dass sich diese durch die Projekte stark erhöhte. Diese Effekte waren bei den Fallstudien sowohl auf der Ebene der Geschäftsführung als auch bei den Mitarbeiterlnnen zu beobachten.

Es hat noch mehr Bewusstsein geschaffen und Diskussionen angeregt.

Manche Zugänge sind anders geworden, weil einem das mehr bewusst geworden ist.

Grafik 44 | Steigerung der Akzeptanz und Awareness für das Thema Gleichstellung im Unternehmen, Anzahl der befragten Organisationen

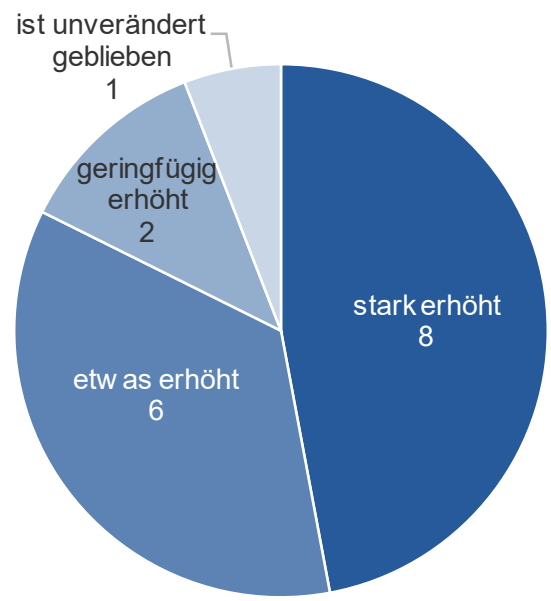

Quelle: FEMtech Karriere Befragung 2020, KMU Forschung Austria, n=17

Im Zuge der Projektumsetzung wurden diverse Produkte und Tools, wie Gender- Diversity-Leitfäden, Leitfäden für MitarbeiterInnen- und Karrieregespräche, Informationsmaterialien zum Thema Gender sowie für neue Mitarbeiterlnnen oder Bewerbungs- und Recruitingtools entwickelt. Auch Monitoring-Instrumente, Nachhaltigkeitsreporting-Tools, Studien und Weiterbildungskonzepte sind im Rahmen einzelner FEMtech Karriere Projekte entstanden. 
Laut Auskunft der befragten ProjektteilnehmerInnen kommen vor allem die entwickelten Informationsmaterialien für neue MitarbeiterInnen sowie die Leitfäden für MitarbeiterInnengespräche und zur weiteren Karriereplanung häufig zum Einsatz. Auch auf neu konzipierte Bewerbungs- und Recruitingtools wird im beruflichen Alltag oft zurückgegriffen. Ebenso werden die Gender- und Diversity Leitfäden häufig von jenen Organisationen genutzt, die solche Instrumente erstellt haben. Allgemeine Informationsmaterialien zum Thema Gender und gendergerechter Sprache finden im täglichen Gebrauch hingegen deutlich seltener ihre Anwendung.

Grafik 45 | Einsatz von im Rahmen von FEMtech Karriere entwickelten Produkten und Tools, Anzahl der befragten Organisationen

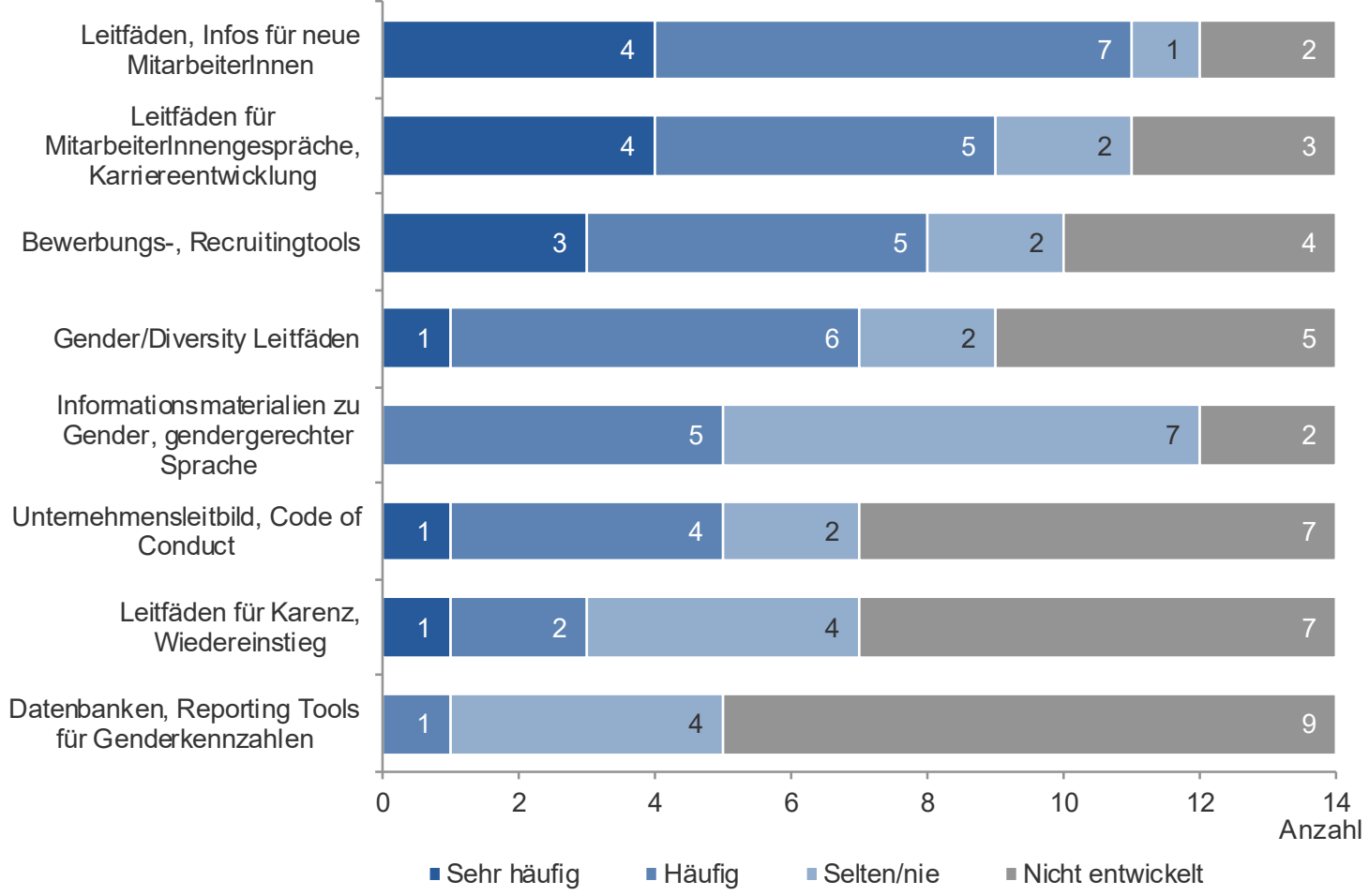

Quelle: FEMtech Karriere Befragung 2020, KMU Forschung Austria, n=14

Auch die Fallstudien zeigen, dass viele entwickelte Produkte häufig zum Einsatz kommen und als Inspirationsquelle für weitere Projekte dienen, während manche nur bei Bedarf herangezogen werden.

Ja, ist schon nachhaltig. Es hat schon etwas gebracht. Wir schauen heute noch gerne in die Unterlagen hinein und lassen uns von diesen Dingen inspirieren.

Die Produkte sind in unseren Alltag integriert und werden weiterhin eingesetzt. Für mich persönlich waren die Leitfäden das Highlight, davon profitiere ich am meisten.

Die Produkte werden schon immer wieder genutzt. Nicht sehr oft, es ist aber gut, dass man es hat. Es schadet nicht, immer wieder hineinzuschauen. 
Mit Hilfe der FEMtech Karriere Projekte wurde nicht nur ein Produktportfolio generiert, es haben sich auch neue Formen der Vernetzung und des Austausches innerhalb der beteiligten Organisationen entwickelt, die noch weiterhin Bestand haben, wie folgendes Beispiel zeigt.

Wir haben jetzt noch immer solche FEM Club Jours fixes, wo sich intern alle Frauen zusammensetzen und austauschen. Das ist im Zuge des FEMtech Karriere Projekts eingeführt worden und das haben wir beibehalten. Wir haben dafür auch die Unterstützung der Geschäftsleitung.

Um die Ergebnisse der FEMtech Karriere Projekte bzw. des FEMtech Karriere-Checks für KMU einem größeren Personenkreis zugänglich zu machen und breiter zu streuen, wurden folgende Aktivitäten im Bereich der Öffentlichkeitsarbeit gesetzt: In erster Linie haben die befragten ProjektteilnehmerInnen Informationen und Ergebnisse ihrer Projekte auf ihrer Website veröffentlicht, wie Grafik 46 zeigt. Auch Onlineplattformen und -websites, wie z. B. open4innovation oder die FFG-Projektdatenbank, werden häufig genutzt bzw. ist deren Nutzung geplant, um über das Projekt zu informieren. Öffentliche Veranstaltungen bieten für viele eine weitere Möglichkeit, um Projektinformationen zu streuen. Ein Teil der beteiligten Organisationen nutzt auch Social Media Kanäle, um über die FEMtech Karriere Projekte bzw. den FEMtech Karriere-Check für KMU zu informieren.

Grafik 46 | Art der durchgeführten oder geplanten Öffentlichkeitsarbeit im Rahmen von FEMtech Karriere bzw. des FEMtech Karriere-Checks für KMU, Anzahl der befragten Organisationen

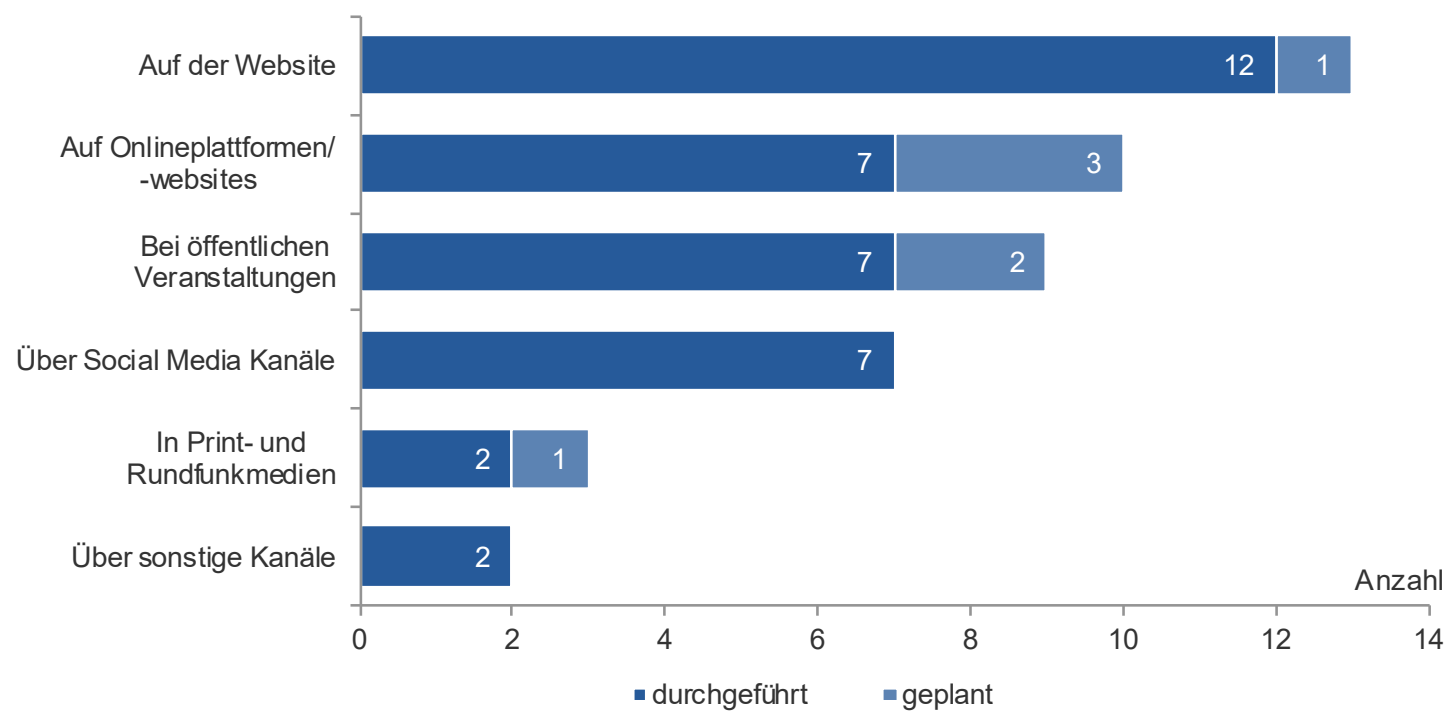

Quelle: FEMtech Karriere Befragung 2020, KMU Forschung Austria, n=15

Insgesamt werden die FEMtech Karriere Projekte und der FEMtech Karriere-Check für KMU von den Fördernehmerlnnen großteils sehr gut oder gut benotet. 6 von 16 ProjektteilnehmerInnen planen sogar noch ein weiteres FEMtech Karriere Projekt einzureichen, um an dem Thema weiterzuarbeiten, die Hälfte weiß es noch nicht. Die Zufriedenheit mit dem Förderangebot ist somit sehr hoch und es trägt maßgeblich zu nachhaltigen strukturellen Veränderungen in den beteilig- 
ten Organisationen bei. Vor allem die Installierung von Gleichstellungs- oder Genderbeauftragten, weitere Verbesserungen der Arbeitsbedingungen und der Unternehmenskultur führen zur Optimierung der Position als attraktive/r ArbeitgeberIn, die auch von den Aktivitäten in der Öffentlichkeitsarbeit gestützt wird. Auch die ExpertInnen sehen die Förderung als eine sehr wichtige Maßnahme an, die viel zur Bewusstseinsbildung beiträgt. Dennoch werden in folgenden Bereichen Verbesserungsmöglichkeiten gesehen.

Die Einstellung des FEMtech Karriere-Checks für KMU, der nur in den Jahren 2015 und 2016 angeboten wurde, ist nicht ganz nachzuvollziehen, da laut FFG noch weiterhin Nachfrage nach diesem Förderangebot besteht. Vorteil dieser Förderung ist die gezielte Ansprache breiterer Unternehmenskreise, die mit Genderthemen und der Abwicklung solcher Förderprojekte noch weniger vertraut sind. Gerade für diese Unternehmen war der FEMtech Karriere-Check für KMU ein guter Einstieg und niederschwelliger Anstoß, Aktivitäten in dieser Richtung zu setzen und eine Genderanalyse und -schulung durchzuführen, auf der im anschließenden FEMtech Karriere-Projekt aufgebaut werden konnte. Die komplexe Modulstruktur der FEMtech Karriere Projekte stellt für Unternehmen, die mit der Thematik noch nicht so vertraut sind, oft eine große Herausforderung dar. Oftmals fehlen auch die (finanziellen) Ressourcen, um solche umfassenden Genderanalysen vorab in Eigenregie durchzuführen. Daher wäre bei einer neuerlichen Aktivierung der Unterstützungsleistung eine 100-ige Förderquote wünschenswert.

Verbesserungsvorschläge zur weiteren Optimierung der FEMtech Karriere Projekte betreffen die komplexe Modulstruktur. Eine Reduktion der Anforderungen bei den Modulen und mehr Offenheit für neue Ansätze und Themen (z. B. Diversity) würden laut ExpertInnenmeinung das Einreichprozedere erleichtern. Auch das Angebot kleinerer Projekte mit geringeren Fördersummen, die auch mit einem niedrigeren Aufwand für die Antragsstellung und Berichtslegung einhergehen, sowie eine Reduktion der Berichtsaktivitäten würden laut befragten Projektteilnehmerlnnen Vereinfachungen darstellen. Ein weiterer Vorschlag, der die Einreichaktivitäten forcieren könnte, wäre eine (finanzielle) Unterstützung bei der Antragerstellung für kleine KMU. Auch die Schaffung einer Möglichkeit, die Projekte in Kooperation mit anderen Organisationen als Kooperationsprojekt abwickeln zu können, würde insbesondere unerfahreneren Unternehmen den Zugang zu diesem Förderangebot erleichtern.

Weitere Anregungen betreffen eine höhere Fördersumme und mehr Budget für eine externe Begleitung der Maßnahmen im Sinne des Change Managements. Außerdem wird für die FEMtech Karriere Projekte eine 100-ige Förderquote oder zumindest eine 100-ige Förderung der externen Kosten für die GenderexpertInnen als sinnvoll erachtet.

Auch hinsichtlich der Messbarkeit der Ziele und der Nachhaltigkeit der Aktivitäten zeigt sich noch Optimierungspotential. Um konkrete Veränderungen messen zu können, ist eine genaue Analyse der Ausgangssituation im Unternehmen im Rahmen des Projektantrags unerlässlich. Anhand dieser lässt sich auch der Innovationsgrad und die Adäquatheit der geplanten Interventionen besser beurteilen. Die ExpertInnen würden sich wünschen, dass mit Hilfe von FEMtech Karriere noch mehr strukturelle Veränderungen in den beteiligten Organisationen erreicht werden und die Akti- 
vitäten noch stärker in die Tiefe gehen und nicht auf oberflächlichen Optimierungen der Außenwirkung beschränkt bleiben. Eine Möglichkeit um das zu forcieren wäre, finanzielle Boni für die Zielerreichung im Bereich der Hard Facts, z. B. der Steigerung der Frauenanteile, zu vergeben.

Um weitreichendere Veränderungen zu erzielen, gilt es auch die Bekanntheit von FEMtech Karriere weiter zu steigern. Vielen Unternehmen ist die Förderung gänzlich unbekannt und sie erkennen den Nutzen in der Forcierung der Chancengerechtigkeit nicht. Auch die ExpertInnen nehmen FEMtech Karriere eher als ein internes Programm wahr, das nur im Forschungsbereich gut sichtbar ist, jedoch in der breiten Öffentlichkeit noch über zu wenig Präsenz verfügt. Daher ist es für die befragten Projektteilnehmerlnnen und ExpertInnen wesentlich, mehr Werbung für FEMtech Karriere Projekte zu machen.

Vielleicht könnte man es noch mehr bewerben. Ich weiß nicht, ob wenn man nicht gezielt danach sucht, es einem unterkommen würde. Über welche Kanäle auch immer. Es wäre sicher noch mehr drinnen, wenn es bekannter ist.

Dabei könnte auch der NutzerInnenkreis auf technologieorientierte, innovative Unternehmen erweitert werden. Die Kontaktherstellung zu Unternehmen könnte beispielsweise durch direkte Ansprache der Geschäftsführungen, in Kooperation mit UnternehmensberaterInnen oder durch die Bereitstellung von Testimonials erfolgen. Wichtig ist vor allem, die praktische Anwendbarkeit und den Erfolg einzelner Projekte beispielhaft als Good Practice darzustellen, um auf konkreten Anwendungsmöglichkeiten aufbauen zu können. Beispielprojekte könnten multimedial in Form von Geschichten präsentiert und gezielt an die potentiellen Zielgruppen adressiert werden.

Ein anderer Ansatz, um mehr Unternehmen dafür zu gewinnen, könnte auch sein, den Themenbereich breiter zu fassen und anstelle des Genderfokus, allgemein die Gewinnung von Talenten und Fachkräften in den Vordergrund zu stellen. Die Förderung könnte als Unterstützungstool positioniert werden, um Unternehmen und Forschungsorganisationen fit für die Belegschaft der Zukunft (d. h. inklusiv und divers) zu machen, indem sie faire und angenehme Arbeitsbedingungen für möglichst viele potentielle Arbeitskräfte schaffen. Diese Attraktivierung der Arbeitsbedingungen und Bemühungen um zukünftige Fachkräfte sprechen breite Kreise von Nachwuchskräften an, haben aber natürlich auch eine Sogwirkung auf Frauen. Ein anderer Ansatz wäre, den Fokus mehr auf Unternehmenskarrieren und Weiterbildung zu legen, um die vorhandene Belegschaft und dabei vor allem die Frauen gezielt bei ihrer Karriereplanung zu unterstützen.

Um generell mehr Frauen für Forschung und Technik zu gewinnen, wird von den befragten ProjektteilnehmerInnen Sensibilisierungs- und Öffentlichkeitsarbeit als sehr wichtig erachtet (siehe Grafik 47). Insbesondere der Bewusstseinsbildung an Schulen kommt eine sehr wichtige Bedeutung zu, aber auch in Unternehmen und in Hochschulen sind weitere Sensibilisierungsaktivitäten gefragt. Ein weiterer wichtiger Faktor sind flexiblere Arbeitsbedingungen in den Unternehmen durch Home-Office, flexible Arbeitszeiten und Teilzeitangebote, die den Frauen die Vereinbarkeit von Beruf und Familie erleichtern. Auch die Karriereplanung und die Work-Life-Balance sollte in den Unternehmen einen höheren Stellenwert einnehmen. Um den Zugang zu Forschung und Technik vor allem für junge Frauen zu erleichtern, wird die stärkere Forcierung von Prakti- 
kumsplätzen für Schülerinnen und Studentinnen als (sehr) wichtige Maßnahme angesehen. Gerade praktische Einblicke in diese Berufsfelder können dazu beitragen, Berufswege in Forschung und Technik einzuschlagen.

Grafik 47 | Wichtige Maßnahmen zur Gewinnung von mehr Frauen für Forschung und Technik, Anzahl der befragten Organisationen

Sensibilisierungs-/Öffentlichkeitsarbeit

Bewusstseinsbildung in Schulen

Flexiblere Arbeitsbedingungen

Bewusstseinsbildung in Unternehmen

Forcierung von Praktikumsplätzen für Studentinnen

Forcierung von Praktikumsplätzen für Schülerinnen

Gendergerechte Bewerbungsprozesse

Bewusstseinsbildung in Universitäten/Hochschulen

Karriereplanung in den Unternehmen

Stärkerer Fokus auf Work-Life Balance in den Untermehmen

Mehr finanzielle Förderungen

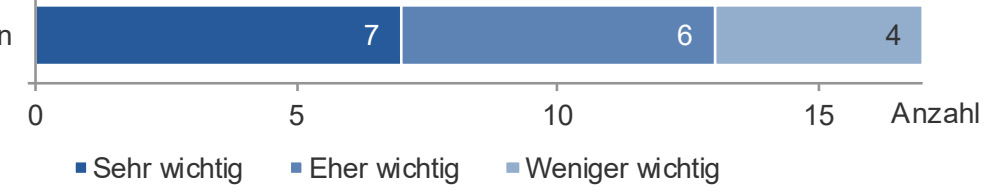

Quelle: FEMtech Karriere Befragung 2020, KMU Forschung Austria, n=17 


\section{5 | FEMtech Forschungsprojekte}

FEMtech Forschungsprojekte ähnelt anderen Förderungen des FFG Portfolios insofern, als dass es wie viele andere Programme F\&E Projekte fördert. Das Alleinstellungsmerkmal gegenüber anderen Projektförderungen besteht in der starken Aufwertung der Genderdimension, die für eine Förderung essentiell ist. So werden ausschließlich Projekte mit genderrelevanten Inhalten gefördert, in denen die jeweilige Projektleitung zumindest Genderkompetenz aufweist und Genderexpertise im Projektteam vorhanden sein bzw. extern eingebunden werden muss.

Tabelle 6 | Eckpunkte zu FEMtech Forschungsprojekte

\begin{tabular}{|c|c|}
\hline $\begin{array}{l}\text { FEMtech For- } \\
\text { schungsprojekte }\end{array}$ & Kurzbeschreibung \\
\hline \multirow{4}{*}{ Ziele } & $\begin{array}{l}\text { FEMtech Forschungsprojekte sollen Vorhaben in Forschung, Technologie und In- } \\
\text { novation mit genderrelevanten Inhalten initiieren. }\end{array}$ \\
\hline & $\begin{array}{l}\text { Es sollen zukunftsrelevante Forschungsfelder und Produkte mit konkreter Gender- } \\
\text { dimension in die Wege geleitet werden. }\end{array}$ \\
\hline & $\begin{array}{l}\text { Die Akzeptanz und das Interesse für das Thema Gender in Forschungsprojekten } \\
\text { soll bei den Wissenschaftlerinnen und Wissenschaftlern und forschungsorientierten } \\
\text { Unternehmen gesteigert werden. Damit soll eine Erhöhung der Qualität von Tech- } \\
\text { nologien und Produkten am Markt sowie die ökonomische Erfolgsperspektive er- } \\
\text { reicht werden. Passgenaue, innovative Lösungen sollen entwickelt werden, die für } \\
\text { andere ideenstiftend sind und Demonstrationscharakter aufweisen können. }\end{array}$ \\
\hline & $\begin{array}{l}\text { Erfolgreiche Projekte führen zu einem besseren Verständnis für genderrelevante } \\
\text { Forschung bzw. einer höheren Innovationskraft und Produktivität durch "Gender- } \\
\text { Balanced Teams“. }\end{array}$ \\
\hline Zielgruppen & $\begin{array}{l}\text { Unternehmen, außeruniversitäre Forschungseinrichtungen; nur kooperative F\&E } \\
\text { Projekte: Universitäten und Fachhochschulen sowie deren Transferstellen }\end{array}$ \\
\hline Inhalt & $\begin{array}{l}\text { Förderung von genderrelevanten F\&E-Projekten, es werden nur Projekte gefördert } \\
\text { die Genderkompetenz, Genderexpertise aufweisen und ein Genderbegriff/-modell } \\
\text { definiert haben. Die durchgängige Einbindung der Genderexpertise ist sicherzustel- } \\
\text { len. }\end{array}$ \\
\hline Instrumente & Einzelprojekte C3 I und kooperative F\&E Projekte C4 E-I \\
\hline Einreichverfahren & Calls, sechs Ausschreibungen bis 2019 \\
\hline Laufzeit & mind. 1 bis max. 3 Jahre \\
\hline Förderquote & $\begin{array}{l}\text { max. } 85 \% \text { (ab der } 5 \text {. Ausschreibung), abhängig vom Organisationstyp und der For- } \\
\text { schungskategorie (Industrielle Forschung oder Experimentelle Entwicklung) }\end{array}$ \\
\hline Förderhöhe & $\max . € 300.000$ pro Projekt \\
\hline Spezifika & $\begin{array}{l}\text { Folgende Themenbereiche sind förderbar: Digitale Technologien, Mobilität und Luft- } \\
\text { fahrt, Energie und Umwelt, Industrielle Technologien, Sicherheitsforschung, Welt- } \\
\text { raumtechnologien, bis zur } 5 \text {. Ausschreibung auch Life Sciences }\end{array}$ \\
\hline
\end{tabular}




\subsubsection{Struktur der geförderten FEMtech Forschungsprojekte und der Projektteilnehmerlnnen}

Im Beobachtungszeitraum von 2011 bis 2019 gab es insgesamt sechs Ausschreibungen in der Programmlinie FEMtech Forschungsprojekte. Von der ersten bis zur sechsten Ausschreibung wurden insgesamt 231 Projekte eingereicht, davon wurden 52 (22,5 \%) gefördert. Pro Ausschreibung wurden 8 (2011-2012) oder 9 (2013, 2014, 2017, 2018) Projekte gefördert. Von den geförderten Projekten wurden alle Projekte der 1. bis 4 . Ausschreibung bereits abgeschlossen, während die geförderten Projekte der 5. und 6. Ausschreibung noch laufen.

Die allermeisten geförderten Projekte $(92,3 \%)$ wurden als Kooperationsprojekte durchgeführt, der Median liegt bei 3 beteiligten Organisationen pro Projekt. Der Anteil der geförderten Projekte lag von 2012 bis 2018 zwischen $17 \%$ und $25 \%$, einzig 2011 wurden $40 \%$ der eingereichten Projekte gefördert (siehe Grafik 48). Insgesamt liegt die Erfolgsquote damit eher im unteren Bereich aller von der FFG verwalteten Förderprogramme, was auf die begrenzten Mittel zurückzuführen ist.

Grafik 48 | Anzahl eingereichter Projekte und Anteil genehmigter Projekte nach Ausschreibungsjahr

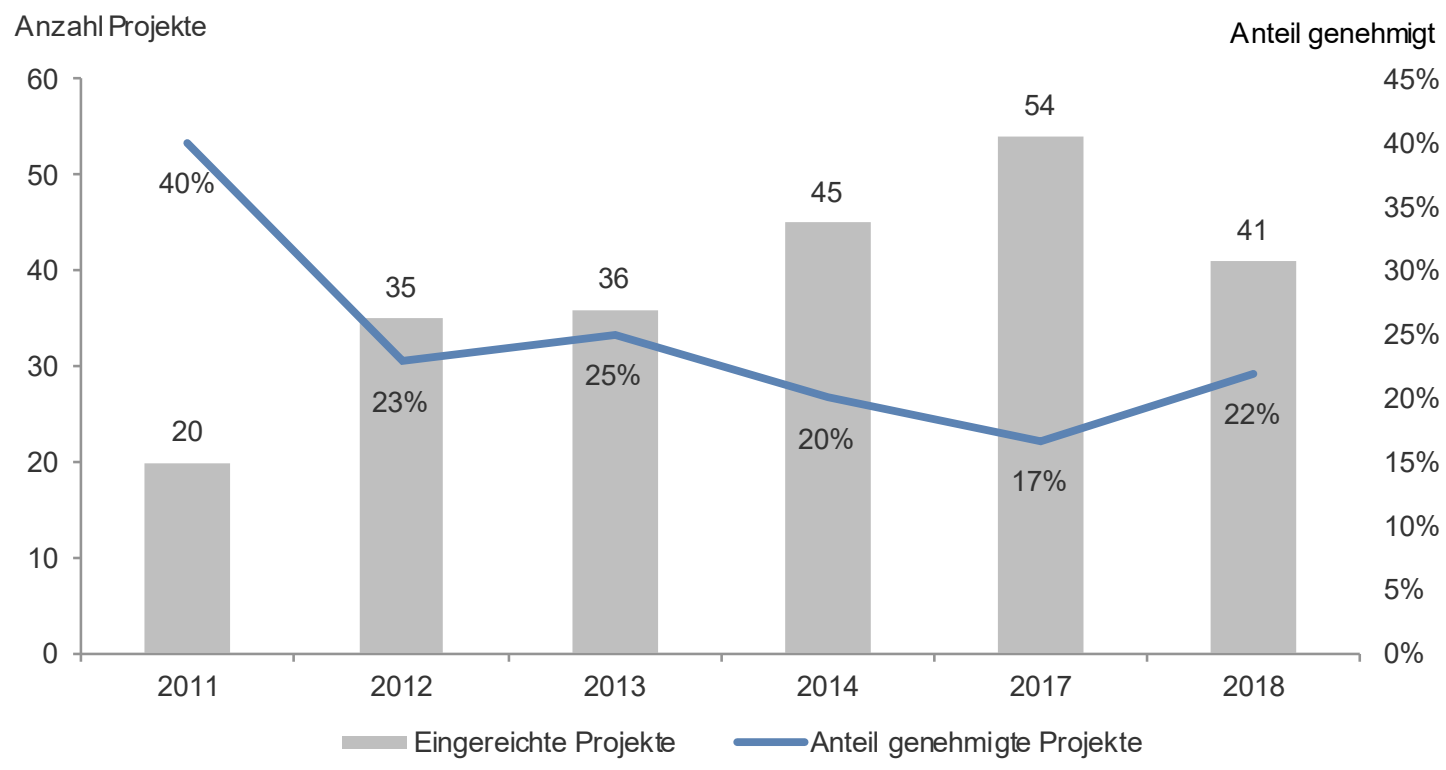

Quelle: FFG; Berechnung und Darstellung KMU Forschung Austria

An den 52 geförderten Projekten waren 110 unterschiedliche Organisationen ${ }^{4}$ beteiligt, einige davon an mehreren Projekten, sodass es insgesamt 173 Beteiligungen an den geförderten Projekten gab. Unternehmen machten den größten Anteil an projektbeteiligten Organisationen aus (siehe Grafik 49).

\footnotetext{
${ }^{4}$ Jede Organisation wurde hierbei nur einmal gezählt.
} 
Grafik 49 | Anzahl der Beteiligungen an FEMtech Forschungsprojekten nach Organisationstyp, 20112018/19

Anzahl Projektbeteiligungen

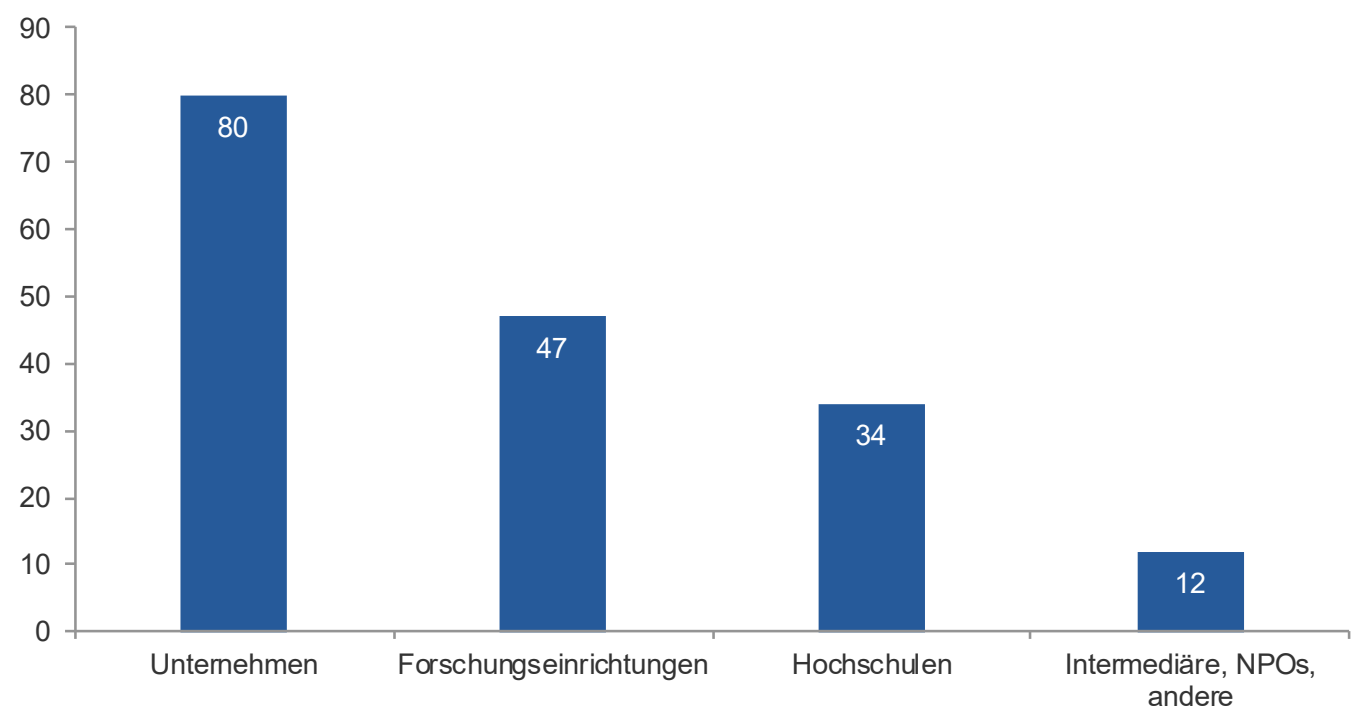

Quelle: FFG; Berechnung und Darstellung KMU Forschung Austria

Der überwiegende Teil der teilnehmenden Organisationen ist in Wien ansässig (59), gefolgt von Organisationen aus der Steiermark (22), und Niederösterreich (10). Auffällig ist, dass keine Organisation aus Vorarlberg in einem FEMtech Forschungsprojekt gefördert wurde, auch die sehr wenigen teilnehmenden Organisationen aus Kärnten (1), Tirol (1) und dem Burgenland (2) bezeugen eine starke Konzentration auf bestimmte Bundesländer. Diese wird noch deutlicher, wenn man den Anteil der eingereichten Projekte an den genehmigten Projekten in Bezug auf den Standort der/s jeweiligen Konsortialführerin/s betrachtet: Von vier eingereichten Projekten mit Salzburger Konsortialführerlnnen wurden drei genehmigt, was $75 \%$ der Einreichungen entspricht. Bei steirischen KonsortialführerInnen beträgt diese „Erfolgsquote“ $28,9 \%$, bei Wiener Organisationen 23,3\% und bei niederösterreichischen Organisationen 20\%. Es gab keine Einreichung mit Konsortialführerlnnen aus dem Burgenland, aus Kärnten, Oberösterreich, Tirol oder Vorarlberg. Grafik 50 stellt die Anzahl der Projektbeteiligungen nach Bundesland dar, wobei beachtet werden muss, dass hierbei eine Organisation (im Falle mehrerer Projektbeteiligungen) auch mehrfach gezählt werden kann. 
Grafik 50 | Anzahl der Projektbeteiligungen nach Bundesland, 2011-2018

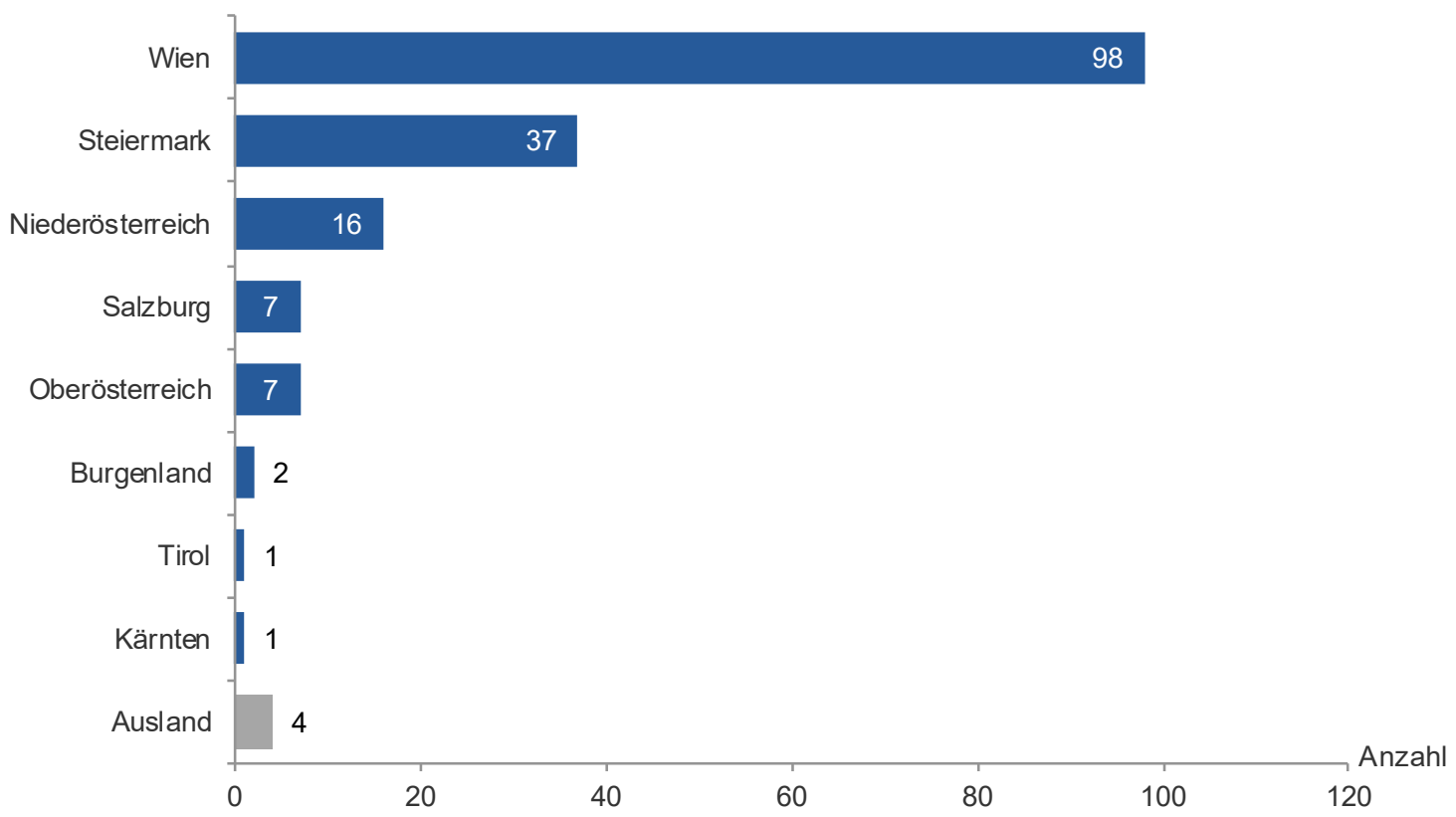

FFG; Berechnung und Darstellung KMU Forschung Austria

Der überwiegende Teil der Organisationen hat im Beobachtungszeitraum ein FEMtech Forschungsprojekt durchgeführt. Grafik 51 zeigt die Anzahl der Organisationen nach ihrer jeweiligen Projektbeteiligung an FEMtech Forschungsprojekten. Für den Organisationstyp Unternehmen zeigt die Grafik folgendes: Von den insgesamt 71 während des Beobachtungszeitraumes an FEMtech Forschungsprojekten beteiligten unterschiedlichen Organisationen, waren 65 nur an einem Projekt beteiligt, 5 Unternehmen an 2 bis 3 Projekten und nur ein Unternehmen an 4 oder mehr FEMtech Forschungsprojekten. Die Darstellungen für die anderen Organisationstypen werden analog gedeutet. Im Vergleich zwischen den Organisationstypen fällt auf, dass es vergleichsweise viele Forschungseinrichtungen gibt, die sich an vier und mehr FEMtech Forschungsprojekten beteiligen bzw. beteiligt haben. 
Grafik 51 | Anzahl der Projektbeteiligungen pro Organisation, 2011-2018, nach Organisationstyp

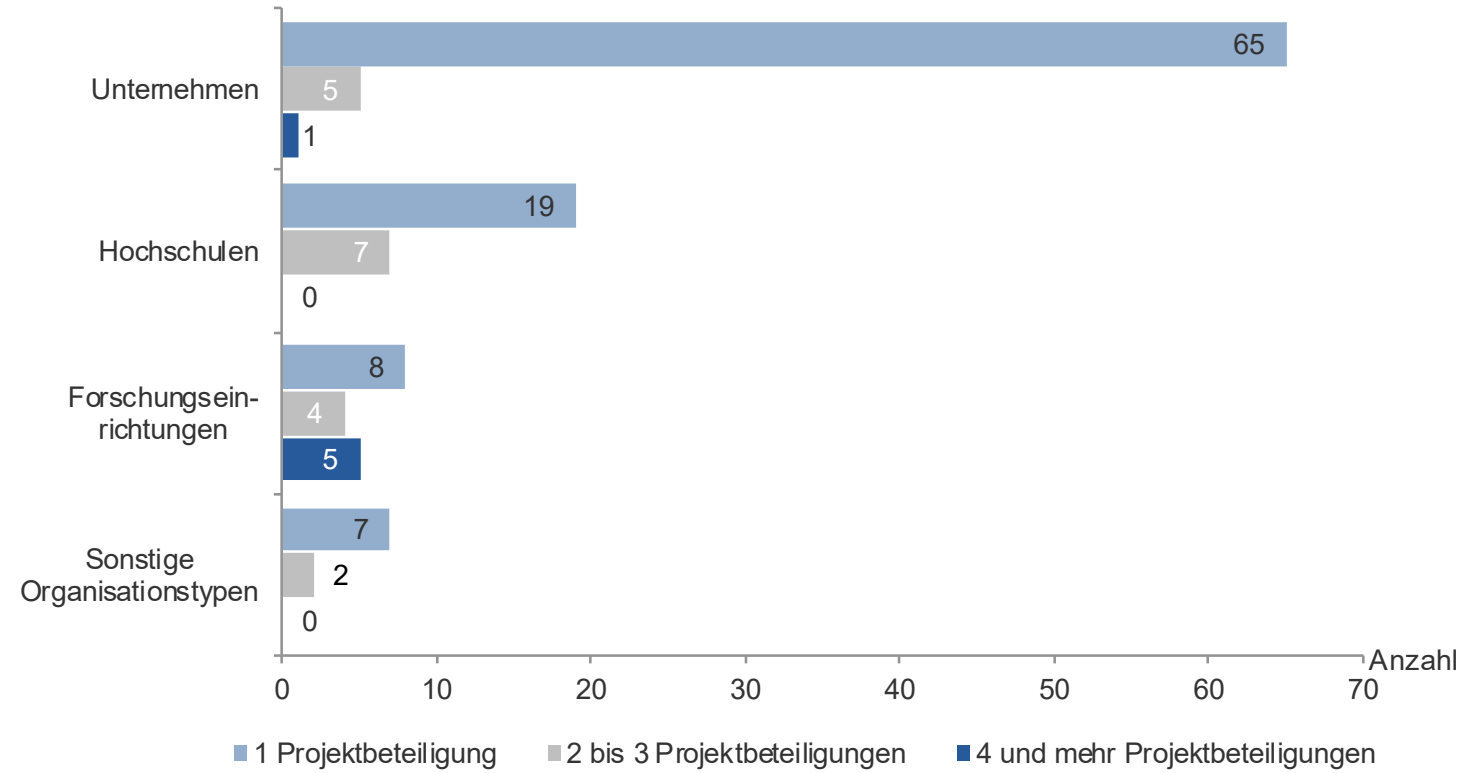

Quelle: FFG; Berechnung und Darstellung KMU Forschung Austria

Die meisten der geförderten Projekte lassen sich dem Themenbereich IKT zuordnen, auch Mobilität, Energie / Umwelt und dem seit der fünften Ausschreibung nicht mehr förderbaren Themenbereich Life Sciences sind häufiger Projekte zugeordnet. Diese Themenbereiche entsprechen den Vorgaben des BMK, eine Themenoffenheit der Programmlinie ist prinzipiell besser geeignet, möglichst viele potentielle FördernehmerInnen anzusprechen und die Programmziele dadurch effektiver erreichen zu können, was heißt: je mehr unterschiedliche Themenbereiche gefördert werden, desto breiter können Gendersensibilität, -relevanz und -kompetenz in allen naturwissenschaftlich und technisch orientierten Organisationen verankert werden. 
Grafik 52 | Verteilung der geförderten FEMtech Forschungsprojekte nach Themen, 2011-2018

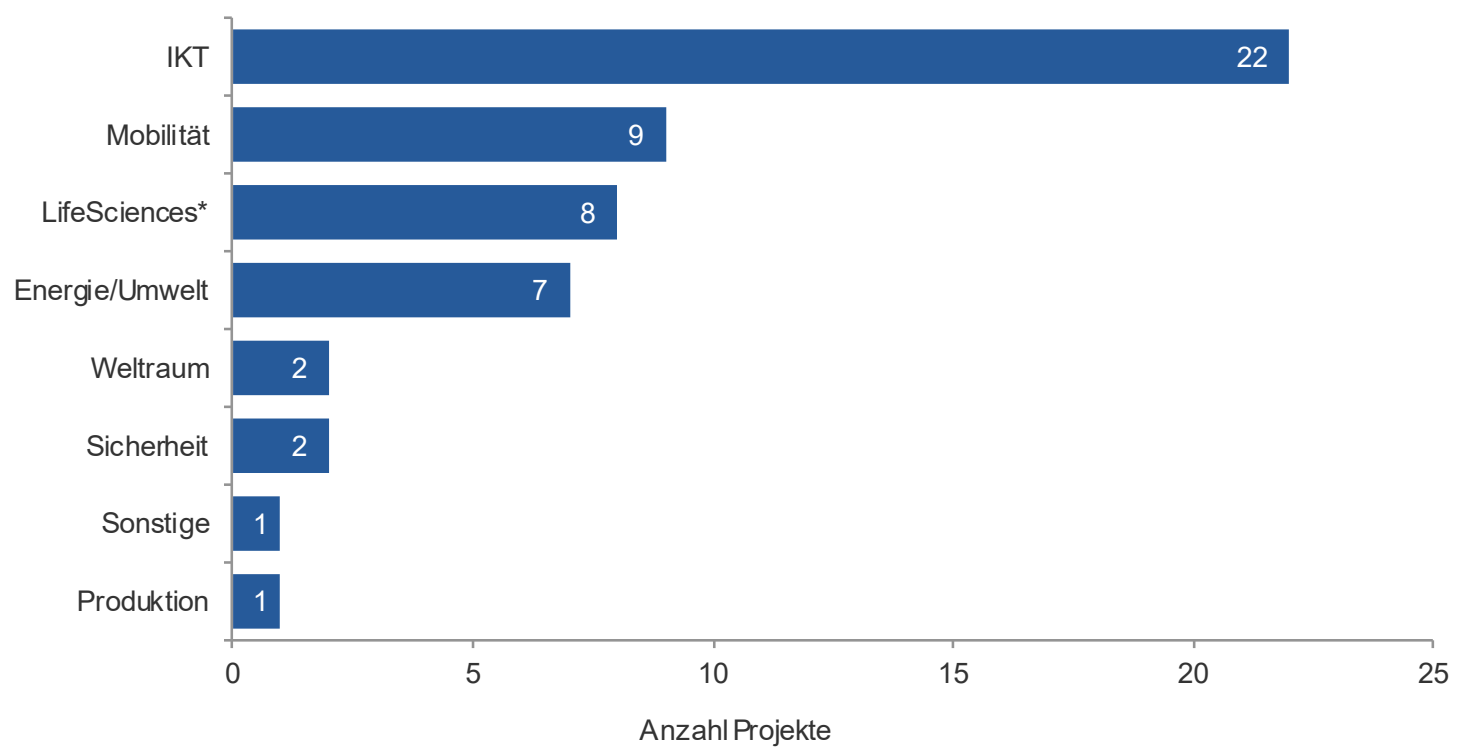

*Einreichung zum Thema Life Sciences war nur bis einschließlich der vierten Ausschreibung möglich Quelle: FFG; Berechnung und Darstellung KMU Forschung Austria

Der Anteil an Organisationen, die erstmals im Programm Talente gefördert wurden und für die FEMtech Forschungsprojekte somit das erste in Talente geförderte Projekt war, liegt je nach Ausschreibung zwischen $44 \%$ und $75 \%$, wie Grafik 53 zeigt. Insgesamt beträgt der Anteil der Erstgeförderten in Talente bei den an FEMtech Forschungsprojekten beteiligten Organisationen bei rd. 54 \%. Der Anteil der Organisationen, welche überhaupt das erste Mal im Rahmen eines FEMtech Forschungsprojekts bei der FFG eingereicht haben, beträgt insgesamt $11 \%$, der Anteil der erstmals von der FFG geförderten Organisationen unter den FEMtech Forschungsprojekte Teilnehmenden beträgt $19 \%$. 
Grafik 53 | Anteil der erstmals in Talente geförderten Organisationen an allen in FEMtech Forschungsprojekte teilnehmenden Organisationen nach Ausschreibungsjahr, in Prozent

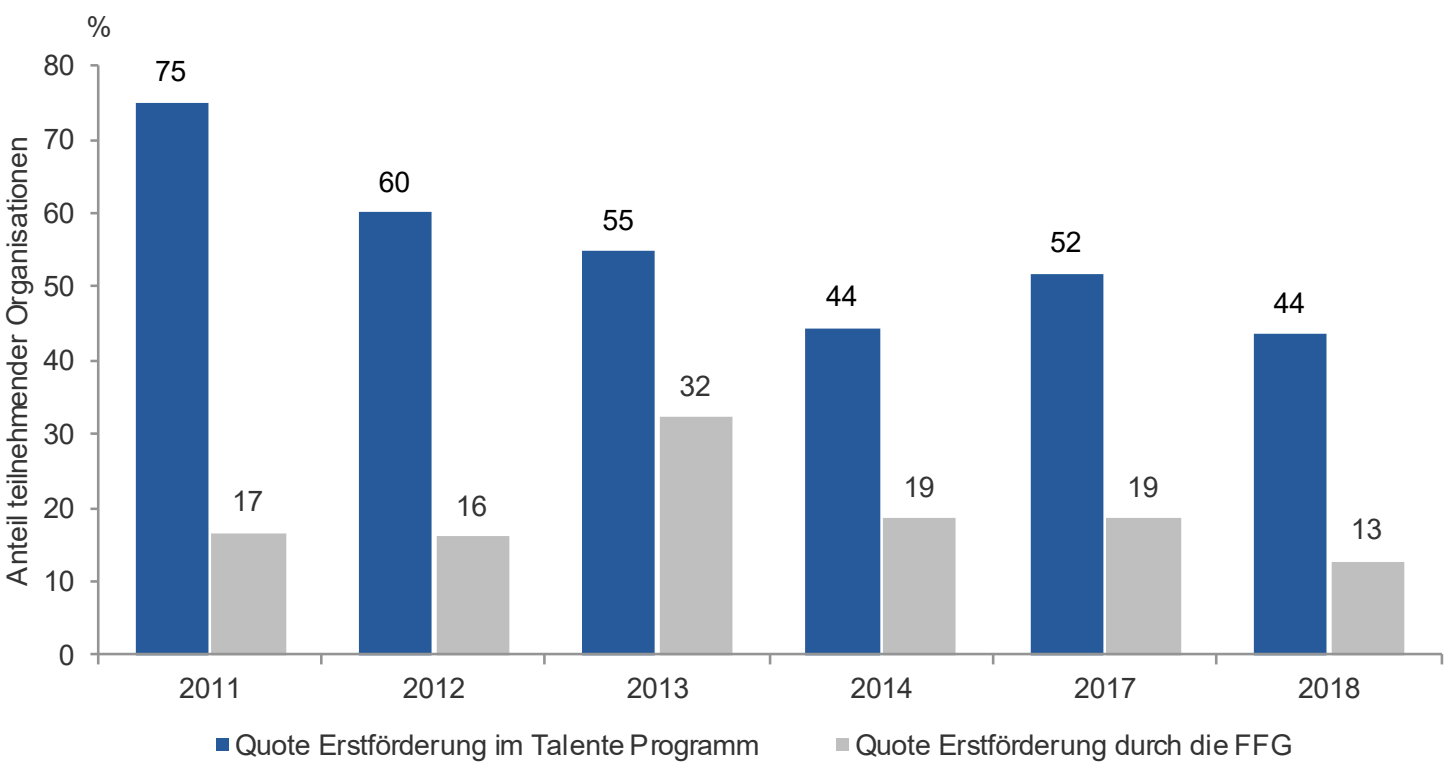

Quelle: Quelle: FFG; Berechnung und Darstellung KMU Forschung Austria

Insgesamt wurden den antragstellenden Organisationen Projektförderungen in Höhe von $€ 13.115 .445$ zugesprochen $^{5}$. Von dieser Summe entfallen $31 \%$ auf Unternehmen, $40 \%$ auf Forschungseinrichtungen, $24 \%$ auf Hochschulen und $5 \%$ auf sonstige Organisationstypen. Im Zeitverlauf ist die Summe des Förderbarwerts leicht gestiegen, von rd. € 1,9 Mio bei der ersten Ausschreibung im Jahr 2011 auf rd. € 2,4 Mio bei der sechsten Ausschreibung im Jahr 2018. Der Anteil des Förderbarwerts nach Organisationstyp variiert im Zeitverlauf; insgesamt betrachtet entfällt der höchste Anteil am gesamten Förderbarwert pro Ausschreibung aber - mit Ausnahme der ersten Ausschreibung - jeweils auf außeruniversitäre Forschungseinrichtungen.

Da FEMtech Forschungsprojekte auch eine gewisse „Vorzeigefunktion“ ausüben können, spielt die Dissemination eine wichtige Rolle. So wurden im Zuge des Reviews im Jahr 2016 (siehe Kap. 5.5.3) Einzeldarstellungen aller 2008 bis 2014 geförderten Projekte erstellt und auf der FEMtech Website veröffentlicht. Auch ist positiv hervorzuheben, dass alle in der 6. Ausschreibung (Jahr 2018) geförderten Projekte bereits in der frei zugänglichen FFG Datenbank gelistet sind. Von den neun im Rahmen der 5. Ausschreibung (2017) geförderten Projekte sind sechs in der Datenbank zu finden, und von der 4. Ausschreibung (2014) ist ebenfalls jedes Projekt online abrufbar. Die im Rahmen von FEMtech Forschungsprojekte geförderten Projekt genderATlas, re-ment und MueGen Driving werden zudem auf der open4innovation Website als Erfolgsgeschichten vorgestellt. Disseminationsveranstaltungen wie beispielsweise Netzwerktreffen oder Austauschevents mit internationaler Beteiligung, die es in der Form bis dato noch nicht gibt, könnten ebenfalls angedacht werden.

\footnotetext{
5 Die tatsächliche Höhe der Förderung für genehmigte Projekte kann je nach Projektverlauf von der bei der Förderentscheidung zugesprochenen Förderhöhe abweichen.
} 
Die FEMtech Forschungsprojekte wurden bereits zweimal einer näheren Analyse unterzogen, so bei der Zwischenevaluierung aus dem Jahr 2014 und in einem Review aus dem Jahr 2016. Die Ergebnisse dieser Analysen werden in der Folge kurz dargestellt und deren Empfehlungen und Verbesserungsvorschläge, welche sich auf die Programmlinie beziehen, mit den in den aktuellen Anträgen und Zwischenberichten der 5 . Ausschreibung verglichen.

\subsection{2 | Ergebnisse der Zwischenevaluierung 2014}

Die Zwischenevaluierung aus dem Jahr 2014 (Heckl et al.) hebt hervor, dass die Programmlinie FEMtech Forschungsprojekte eine Lücke in der Förderlandschaft füllt, und die Fördermöglichkeit auch von der Zielgruppe als notwendige Unterstützung wahrgenommen wird, da dadurch viele Projekte durch die finanzielle Unterstützung ermöglicht werden, die ansonsten trotz eines potentiellen hohen gesellschaftlichen Nutzens nicht umgesetzt werden würden. Die geförderten Unternehmen und Forschungseinrichtungen sind insgesamt gesehen sehr zufrieden mit FEMtech Forschungsprojekte. Im Rahmen der Evaluierung wurden auch drei Fallstudien durchgeführt. Diese veranschaulichten unter anderem, dass Genderkompetenz bzw. -expertise schon vor Beginn des Projekts vorhanden war bzw. die Projektbeteiligten schon über Erfahrungen im Themenfeld Gender verfügten. Auch war in den drei näher untersuchten Fällen die FFG und ihr Förderportfolio zumindest teilweise bereits bekannt, es handelte sich also in diesem Sinn nicht um Newcomer. Interessant sind die Fallstudien außerdem, da sie aufzeigen, dass FEMtech Forschungsprojekte ein breiteres Feld als nur Gender abdecken und auch Fragen der Diversität abdecken können. Das Projekt „living gender" ist diesbezüglich besonders interessant, da hier Gendergerechtigkeit als ein Teilbereich von Diversitätsgerechtigkeit verstanden wird, die beispielsweise auch die sozialstrukturellen Merkmale Alter, Behinderung, Migrationshintergrund und Einkommen umfasst. In Bezug auf das Konzept Diversität finden sich somit vielfältige Anknüpfungspunkte an den übergeordneten Förderschwerpunkt Talente. Die Fallstudien zeigten zudem, dass die Projekte, obwohl zum damaligen Zeitpunkt noch nicht abgeschlossen, Lernprozesse bei den beteiligten Organisationen initiierten bzw. zu Austauschprozessen und Wissensaufbau führten. Zusammenfassend wird die Programmlinie als im Rahmen von Talente gut platzierte Förderung gewertet, deren geförderte Projekte die teilnehmenden Organisationen und das Forschungsfeld positiv beeinflussen.

\subsubsection{Ergebnisse des Reviews der FEMtech Forschungsprojekte 2016 und abschließende Beurteilung der Programmlinie}

Das vom Institut für Höhere Studien (IHS) im Auftrag des Bundesministeriums für Verkehr, Innovation und Technologie (BMVIT) erstellte Review der FEMtech Forschungsprojekte 2008 bis 2014 (Wroblewski 2016) hebt die nationale als auch internationale Vorbildfunktion der Programmlinie hervor, gibt aber auch einige Empfehlungen zur Verbesserung der Programmlinie ab. Das Review untersucht insbesondere, ob und inwiefern Gender als Forschungsinhalt in den Projekten behandelt wurde. Das Review hält zum Beispiel fest, dass die Verankerung von Genderexpertise in den Projekten variiert, und nicht immer bei allen am Projekt beteiligten Organisationen Genderexpertise vorhanden ist, sondern gegebenenfalls nur bei einer/m Projektpartnerln oder durch externe GenderexpertInnen eingebracht wird. Auch wird der Genderbegriff in nur etwa einem Drittel der Projektanträge konkret definiert. Obwohl in vielen Fällen keine explizite Definition des 
Genderbegriffs erfolgt, konnte die Autorin aus den Anträgen den dem jeweiligen Genderverständnis zugrundeliegenden theoretische Zugang ableiten, wobei sich Unterschiede nach den jeweiligen Themenbereichen zeigten. Wurde Gender explizit definiert, lag dieser Definition ein sozialkonstruktivistisches Verständnis von Gender zu Grunde, vor allem im Themenfeld Life Sciences hingegen konnte häufiger auf einen differenztheoretischen Zugang zum Genderbegriff geschlossen werden. Auch bei der Einbindung von Personen, die über Genderexpertise verfügen, zeigten sich bei den jeweiligen Projekten Unterschiede, etwa deren Einbezug bei der Antragstellung. Als besonders wichtig für die erfolgreiche Umsetzung der Projekte werden unter anderem Genderexpertise bei der Projektleitung, klare Aufgabendefinitionen und eine gewisse Akzeptanz und Autorität der Genderexpertinnen und -experten genannt. Das Review hebt außerdem hervor, dass 69 \% der Projekte von Frauen geleitet wurden, was deutlich über dem Frauenanteil in der außeruniversitären bzw. naturwissenschaftlich-technischen Forschung liegt.

Die Autorin gibt eine Reihe von Empfehlungen zur Weiterentwicklung der Programmlinie. So plädiert sie bei der Programmausgestaltung für einen auf Expertise abgestellten Zugang und zieht diesen einem niedrigschwelligen Zugang vor, um an den jeweiligen Stand der Genderforschung im betreffenden Themenbereich anschließen zu können. FEMtech Forschungsprojekte sind somit als Forschungsprojekte zu sehen, in denen der Genderaspekt zentral verankert ist, und sollen somit nicht nur in naturwissenschaftlicher oder technischer, sondern auch hinsichtlich Gender an den Stand der aktuellen Forschung anschließen. Auch die eher geringe Erfolgswahrscheinlichkeit von eingereichten Projekten lässt vermuten, dass hier keine klassischen Forschungs- und Entwicklungsprojekte mit einem „Genderanstrich“ gefördert werden, was auch im Rahmen der Fallstudien in der Zwischenevaluierung (Heckl et al. 2014) als auch in den verfügbaren Zwischenberichten zur 5. Ausschreibung bestätigt wurde. Zum einen zeigt sich, dass Gender in den Projekten nicht als "frauenspezifisch“, sondern durchaus umfassender gedacht wird und auch breiter angelegte Diversitätskonzepte (z. B. Alter, sozioökonomischer und kultureller Hintergrund, etc.) miteinschließt, für die eine Analyse bzw. eine Berücksichtigung der Genderdimension einen Nutzen für die jeweilige Zielgruppe hat ${ }^{6}$. Projekte und Forschungsfragen werden somit aus einer interdisziplinären Perspektive betrachtet, was großes Potential für Wissensaustausch und Know-howTransfer bietet, stellt aber zugleich größere Herausforderungen an die Zusammenarbeit im Team und an Übersetzungsleistungen zwischen den einzelnen Fachdisziplinen.

Eine weitere Empfehlung lautet, zwischen Genderexpertise und Genderkompetenz zu differenzieren. Letzteres bedeutet eine Reflexionsbereitschaft der Projektbeteiligten, während ersteres deutlich stärker auf Expertise im Zusammenhang mit Geschlechtertheorien, den aktuellen Forschungsstand, Gender Mainstreaming, sowie von Konstruktionsprozessen von Gender abzielt. Zudem sollten Genderexpertinnen und -experten von Anfang, d. h. bereits bei der Antragstellung, in das Projekt eingebunden werden und auch bei der Bewertung von Anträgen sollte auf die Verankerung von Genderexpertise im Projekt geachtet werden. Auch sollten die Rolle und Funktion der Genderexpertinnen und -experten bereits von Anfang an geklärt sein. Die Reflexion des Forschungsprozesses selbst sollte ebenfalls Teil von FEMtech Forschungsprojekten sein, um einen

\footnotetext{
6 Des Weiteren ist in den Antragsunterlagen die Berücksichtigung der Genderrelevanz über die gesamte Laufzeit eines Projekts darzustellen. Insgesamt gesehen nimmt die Darstellung des Genderaspekts somit eine zentrale Position im Antrag ein.
} 
nachhaltigen Lernprozess bei den Projektbeteiligten zu unterstützen bzw. diesen Lernprozess, der häufig implizit stattfindet, auch zu dokumentieren. Es sollten auch vermehrt Ressourcen für eine nachhaltige Dissemination zur Verfügung gestellt werden, um nachhaltigere Projektergebnisse erzielen zu können. Beispielsweise sollten auch über mehr Projekte Informationen zur Verfügung gestellt werden. Auch regelmäßige Veranstaltungen mit wechselnden thematischen Schwerpunkten könnten die Vernetzung innerhalb der Community unterstützen.

Im Hinblick auf die Empfehlungen aus dem Review ist eine deutliche Änderung der Ausschreibungsleitfäden von der 4. zur 5. Ausschreibung erkennbar. So werden beispielsweise Genderkompetenz und Genderexpertise differenziert beschrieben und als in Projekten umzusetzende Punkte angeführt. Auch ist die Definition des Genderbegriffs bzw. -modells nun im Antrag anzuführen, und die Genderexpertise ist bereits von Beginn an und durchgängig in das Projekt einzubinden. Ebenso ist eine Person mit Genderexpertise im Antrag zu benennen, zu deren Aufgabe es unter anderem zählt, Genderkompetenz an das gesamte Projektteam zu vermitteln. Somit können diese im Review empfohlenen Vorschläge als formal umgesetzt angesehen werden. Aus den Anträgen zur 5. Ausschreibung ist für jedes der neuen geförderten Projekte eine Genderrelevanz erkennbar, auch Genderkompetenz und Genderexpertise werden in den Anträgen berücksichtigt. Genderexpertise ist in allen Projekten - soweit aus den Anträgen ersichtlich - vorhanden. Dies zeigt sich an den beschriebenen Kompetenzen der Projektmitarbeiterlnnen, auch wenn die Genderexpertin / der Genderexperte - wie in drei Fällen - nicht namentlich genannt bzw. formal festgelegt wird. Die Zwischenberichte der 5. Ausschreibung lassen darauf schließen, dass nicht nur erste, genderrelevante Projekterkenntnisse gewonnen wurden, sondern in den meisten Projekten bereits Genderexpertise zwischen den Projektbeteiligten vermittelt werden konnte.

Nicht umgesetzt wurde bis dato hingegen der Vorschlag, weitere Vernetzungs- und Disseminationsveranstaltungen abzuhalten. Zwar finden sich die im Zuge des Reviews erstellten Einzeldarstellungen der 2008 bis 2014 geförderten Projekte auf der FEMtech Website und es werden die aktuelleren FEMtech Forschungsprojekte in der FFG Projektdatenbank gelistet, jedoch könnten im Bereich der Dissemination über Begleitmaßnahmen noch mehr Aktivitäten durchgeführt werden, sofern der FFG hierfür entsprechendes Budget zur Verfügung stünde. 


\section{6 | Karriere-Grants}

Die Programmlinie Karriere-Grants unterscheidet sich von anderen Programmlinien bzw. anderen Programmen im Portfolio der FFG vor allem durch ihren Zielgruppenfokus, der auf EinzelforscherInnen und nicht auf juristischen Personen (Unternehmen, Forschungseinrichtungen, Hochschulen, etc.) liegt. Auch sind die Förderungen nicht an konkrete Projekte gekoppelt, sondern an die jeweiligen Individuen (Reisekostenzuschuss, Zuschuss zu Umzugs- und Integrationskosten).

Tabelle 7 | Eckpunkte zu Karriere-Grants

\begin{tabular}{|c|c|}
\hline Karriere-Grants & Kurzbeschreibung \\
\hline Ziele & $\begin{array}{l}\text { Ziel ist es, Forscherinnen und Forscher aus dem Ausland für die anwendungsori- } \\
\text { entierte Forschung in Österreich zu gewinnen. Dadurch soll Personalengpässen bei } \\
\text { Organisationen in der anwendungsorientierten Forschung entgegengewirkt und } \\
\text { diese im internationalen Wettbewerb um Hochqualifizierte unterstützt werden. }\end{array}$ \\
\hline Zielgruppen & Forscherlnnen im Ausland \\
\hline Inhalt & $\begin{array}{l}\text { Vergeben werden Zuschüsse zu Job Interviews in Österreich (Interview Grant), } \\
\text { Umzüge der ForscherInnen bei Aufnahme eines neuen Beschäftigungsverhältnis- } \\
\text { ses in F\&E\&I in Österreich (Relocation Grant) sowie damit zusammenhängende } \\
\text { Integrationskosten vom/von der Partnerln der ForscherInnen (Dual Career Grant). }\end{array}$ \\
\hline Einreichverfahren & Laufende Einreichung \\
\hline Förderungsquote & $80 \%-100 \%$ \\
\hline $\begin{array}{l}\text { Max. Förderungs- } \\
\text { höhe }\end{array}$ & $\begin{array}{l}\text { Interview Grant: max. } 80 \% \text { der Reise- und Nächtigungskosten (Nächtigung: } € 100 \text { ) } \\
\text { Relocation Grant: } € 2.000 \\
\text { Dual Career Grant: } € 2.000 \text { (vor } 2013 € 1.800 \text {,-, anrechenbare Kostenkategorien } \\
\text { erweitert) } \\
\text { Vor } 2013 \text { Deckelung in unterschiedlichen Kostenkategorien }\end{array}$ \\
\hline $\begin{array}{l}\text { Förderbare Kos- } \\
\text { ten }\end{array}$ & $\begin{array}{l}\text { Interview Grant: Reise- und Nächtigungskosten } \\
\text { Relocation Grant: Anreise- und Umzugskosten, Integrationskosten } \\
\text { Dual Career Grant: Integrationskosten }\end{array}$ \\
\hline Spezifika & $\begin{array}{l}\text { Themenoffen (alle Disziplinen); mind. Niveau eines Masterabschlusses der For- } \\
\text { scherlnnen }\end{array}$ \\
\hline $\begin{array}{l}\text { Bewertungsver- } \\
\text { fahren }\end{array}$ & Formalprüfung, Begutachtung und Entscheidung durch FFG \\
\hline
\end{tabular}

Voraussetzung zur Teilnahme am Programm ist ein Abschluss auf zumindest Masterniveau und eine Bewerbung bei einer Organisation (Unternehmen, Forschungseinrichtung, Hochschule) im Bereich Forschung, Entwicklung und Innovation in Österreich. Im Falle einer erfolgreichen Bewerbung ist für den Relocation Grant zudem ein Arbeitsvertrag/Anstellungsverhältnis von mindestens 12 Monaten in Österreich vorzuweisen. Der Interview Grant und der Relocation Grant können unabhängig voneinander in Anspruch genommen werden, der Dual Career Grant kann nur von PartnerInnen einer/eines Relocation Grant Bezieherin/Beziehers in Anspruch genommen werden. 


\subsubsection{Ansprache der Zielgruppe, Struktur der Einreichungen und verge- bene Förderungen}

Aufgrund der speziellen Zielgruppe gestaltet sich die Ansprache von ForscherInnen, die eine berufliche Tätigkeit in Österreich ins Auge fassen, als Herausforderung. Dies vor allem, da das zeitliche Fenster, in dem eine Förderung für Personen in Frage kommt meist sehr klein ist, und von einer Vielzahl von Faktoren abhängt (Stellenausschreibungen, alternative Angebote, etc.). Die FFG versucht daher vor allem über Plattformen (Euraxess) und über die Ansprache von Unternehmen und Organisationen die Zielgruppe indirekt zu erreichen. Organisationen und Unternehmen können die Informationen dann an potentielle BewerberInnen weitergeben (z. B. auf Karrieremessen).

Auf Basis einer Befragung der Fördernehmerlnnen von Karriere-Grants ist ersichtlich, dass die meisten Personen (65\% der Befragten) unter anderem über die Organisation, bei der sie sich beworben haben, auf die Fördermöglichkeit aufmerksam gemacht wurden. Für beinahe ein Drittel waren Freundinnen bzw. Freunde / Kolleginnen bzw. Kollegen und für jede/n Zehnte das Internet Informationsquellen.

Grafik 54 | Informationsquelle der Geförderten, in Prozent (Mehrfachnennungen möglich)

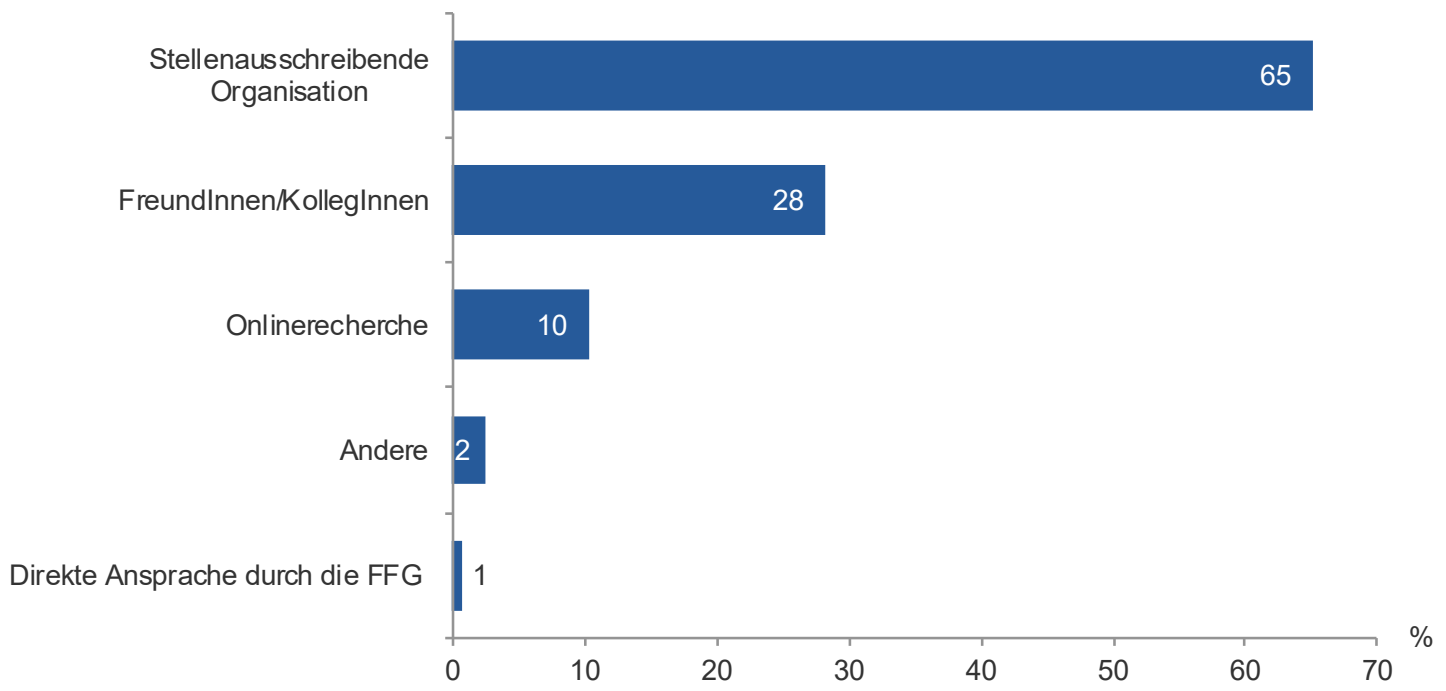

Quelle: Befragung Karriere-Grants FördernehmerInnen, KMU Forschung Austria; n=790

Anträge zu Karriere-Grants werden seit 2019 über das e-Call System der FFG abgewickelt (davor erfolgte die Antragstellung per Zusendung des Antragsformulars an die FFG, beispielsweise über E-Mail). Den Angaben der FFG zufolge erfolgt eine Rückmeldung über eine Fördermöglichkeit innerhalb von 3 bis 5 Tagen. Die Förderung wird im Nachhinein (also nach dem Bewerbungsgespräch bzw. nach dem Umzug nach Österreich ausbezahlt), und basiert auf der Prüfung der von den AntragstellerInnen übermittelten Abrechnungsunterlagen. Die Förderquote ist sehr hoch, wie in Grafik 55 ersichtlich ist. Nur sehr wenige Anträge werden abgelehnt, der überwiegende Anteil der nicht geförderten Anträge geht auf zurückgezogene Anträge zurück. 
Grafik 55 | Anzahl Einreichungen und genehmigte Anträge in Karriere-Grants insgesamt, nach Jahr des Eingangsdatums 2011-2020

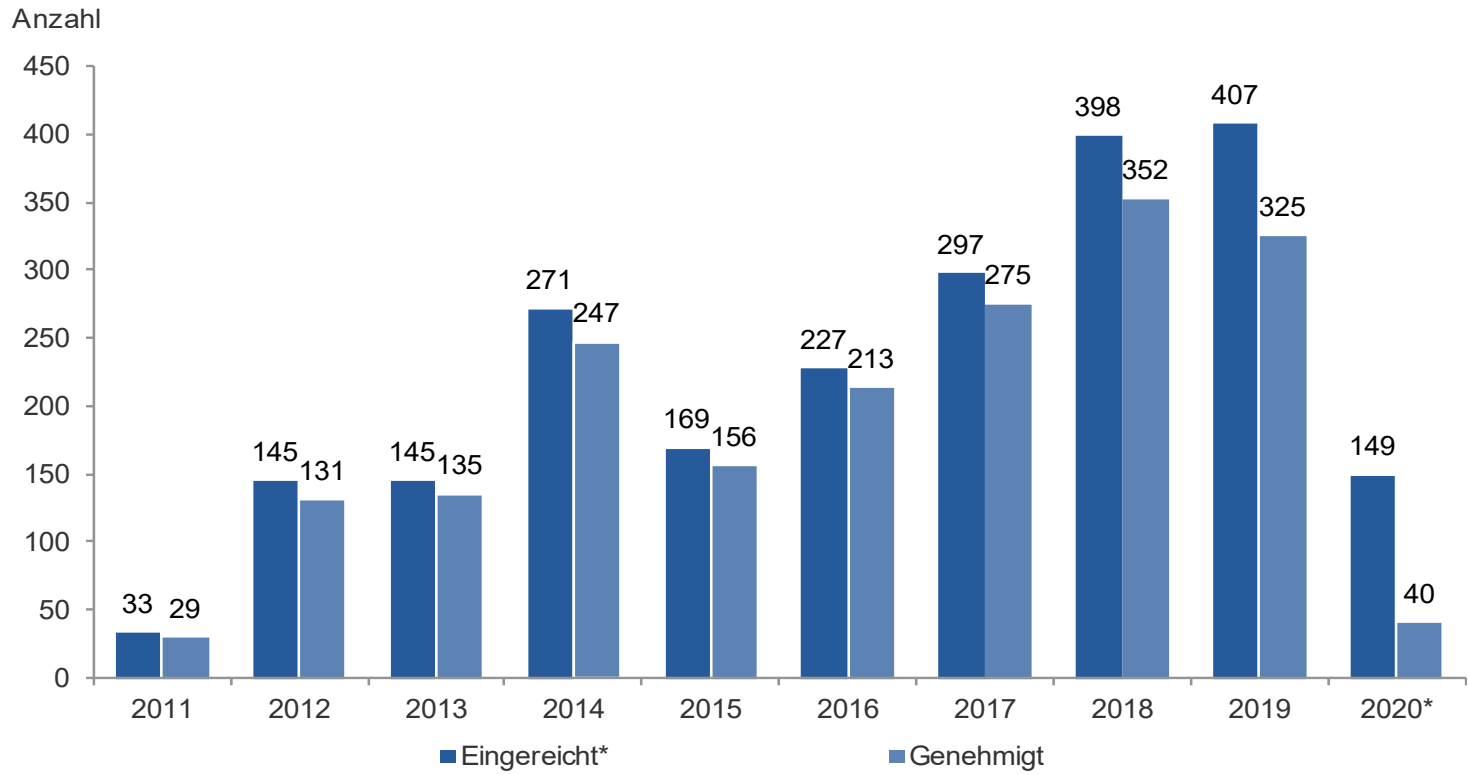

*Einige Einreichungen von vor 2019 wurden nicht in die Datenbank der FFG übernommen, sofern sie auf Basis des EMail-Antrags bereits zuvor abgelehnt wurden. Dies betrifft aber nur wenige (insgesamt 21 Einreichungen von 2011 bis 2018). Für das Jahr 2020 sind die Werte noch unvollständig.

Quelle: FFG, Berechnung und Darstellung KMU Forschung Austria

Insgesamt gab es im Beobachtungszeitraum 2.241 Einreichungen, davon wurden 1.903 gefördert, wobei zum Evaluierungszeitpunkt noch 112 Förderentscheidungen für 2019 in den von der FFG zur Verfügung gestellten Daten offen waren. Diese Ausschreibung musste auch aufgrund einer Ausschöpfung der Mittel erstmals vorzeitig geschlossen werden. Die Verteilung der geförderten EinzelforscherInnen nach Grant-Typ sind in Grafik 56 (links) dargestellt.

Die maximale Förderhöhe beträgt bei Relocation Grants und Dual Career Grants $€ 2.000$ (vor 2013: € 1.800). Für Interview Grants beträgt die Förderquote 80 \%, die höchste Förderung für Interview Grants im Beobachtungszeitraum betrug $€ 1$.644, der Median lag bei $€ 252,5$, was deutlich geringer ist als bei den anderen Grant-Arten. So liegt der Median Wert beim Relocation Grant bei $€ 1.268$ und beim Dual Career Grant bei $€ 1.447,5$. Der Großteil der Kosten von Interview und Relocation Grants wird laut Angaben der FFG für Reisekosten bzw. bei Job Interviews auch für Nächtigungskosten in Anspruch genommen. Bei Dual Career Grants wird die Förderung als Zuschuss vorwiegend für Kinderbetreuungskosten, Integrationskosten und Sprachkurse verwendet.

Insgesamt waren bis zur Ausschreibung 201957 \% der geförderten Einreichungen Relocation Grants, 38 \% Interview Grants, und $5 \%$ Dual Career Grants. Forscher nehmen Karriere-Grants häufiger in Anspruch als Forscherinnen (siehe Grafik 56 rechts). 
Grafik 56 | Verteilung des geförderten Karriere Grant Typs und des Geschlechts der Einzelforscherlnnen, nach Ausschreibungsjahr 2011-2019, in Prozent

\section{Art der Karriere-Grants 2011-2019}

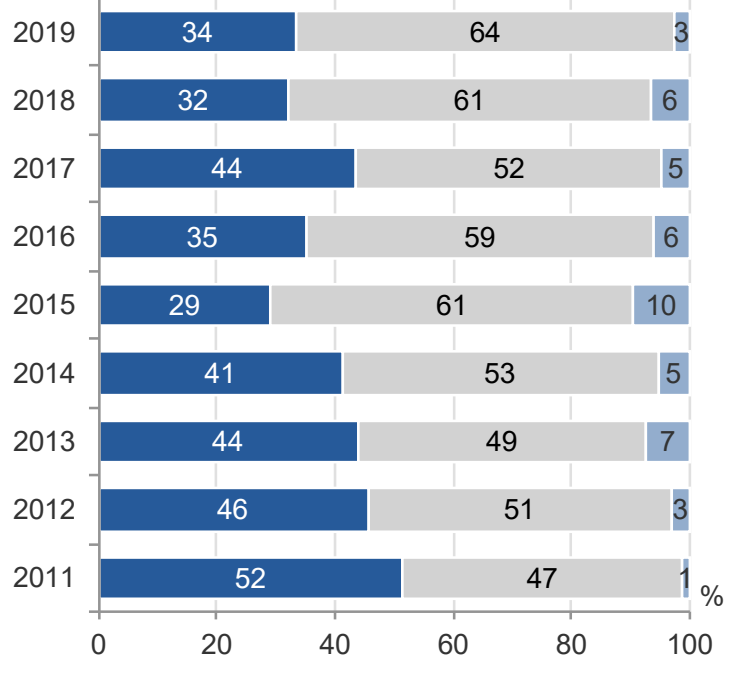

- Interview Grant $=$ Relocation Grant $=$ Dual Career Grant

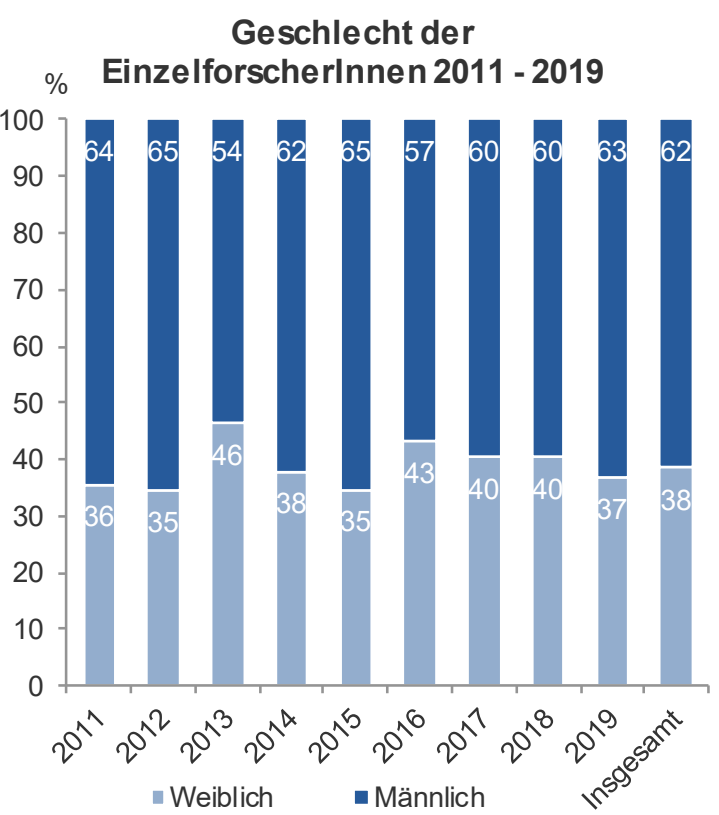

Quelle: FFG, Berechnung und Darstellung KMU Forschung Austria

Grafik 57 gibt die Anzahl der Anträge pro Kalenderjahr nach dem Aufenthaltsort der Antragstellerin bzw. des Antragstellers wider. Der überwiegende Anteil der Personen hielt sich zum Zeitpunkt der Antragstellung in Europa auf. Im Zeitverlauf erkennbar ist auch eine Zunahme der Personen, die sich zum Zeitpunkt der Antragstellung in Asien oder "weiteren Ländern" aufhielten, was darauf schließen lässt, dass das Angebot vermehrt auch von Hochqualifizierten außerhalb Europas und Nordamerikas in Anspruch genommen wird. 
Grafik 57 | Anzahl der Anträge nach Aufenthaltsort der Antragstellerlnnen, nach Kalenderjahr 2012-2019

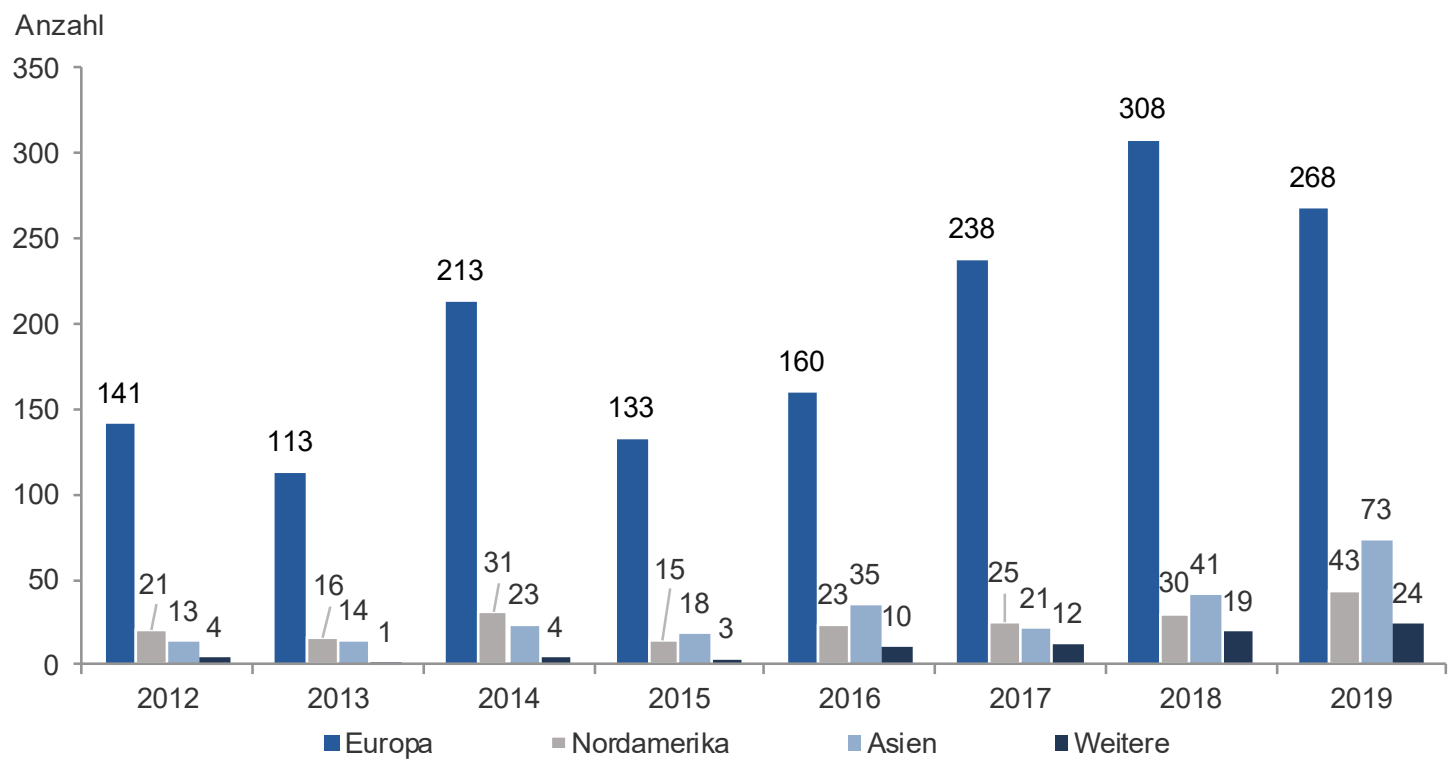

Quelle: FFG Monitoringberichte, Darstellung KMU Forschung Austria

Die StellenanbieterInnen für Personen, die für eine Karriere-Grants Förderung einreichen, konzentrieren sich vor allem auf die Bundesländer Wien, Steiermark und Niederösterreich (siehe Grafik 58).

Grafik 58 | Anzahl der StellenanbieterInnen nach Bundesland, 2011-2019

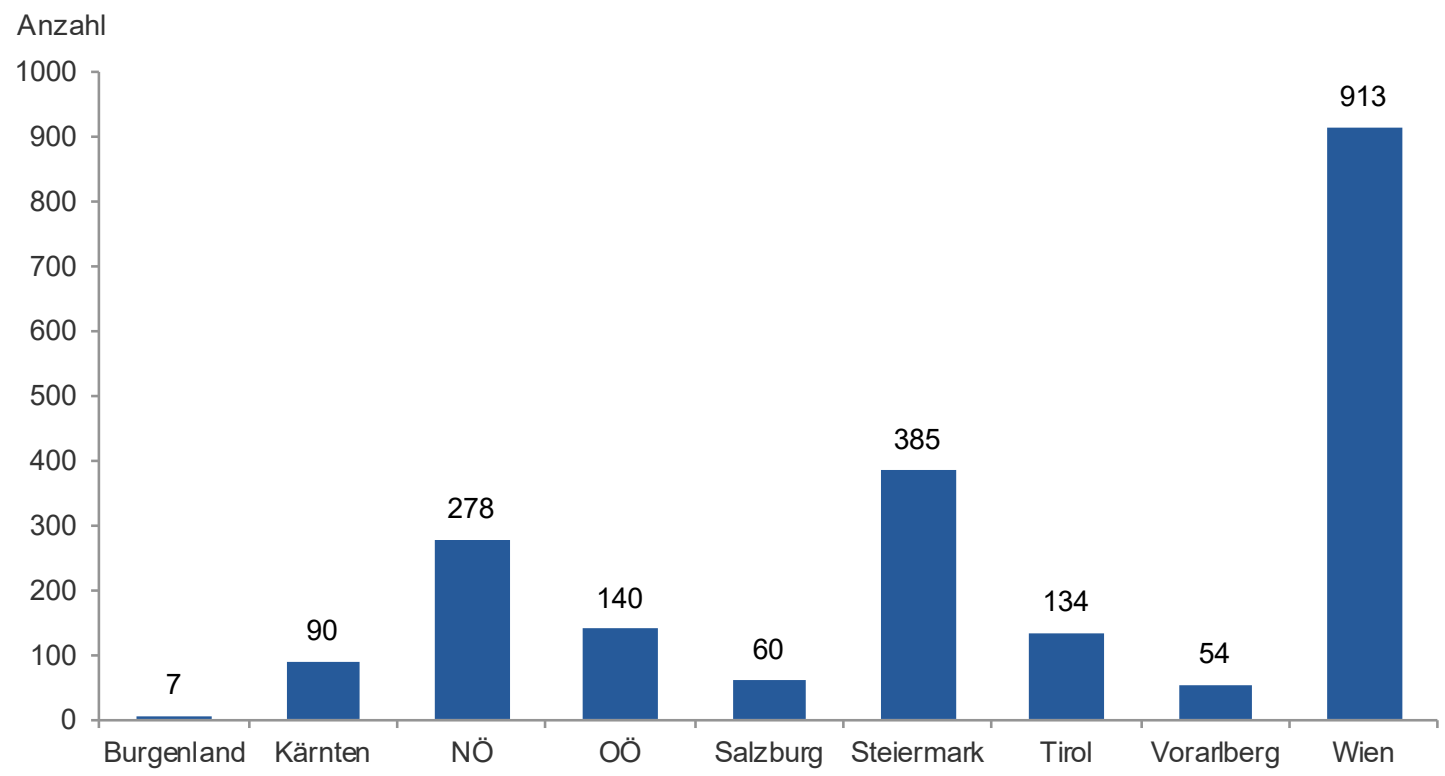

Quelle: FFG Monitoringberichte, Darstellung KMU Forschung Austria 


\subsection{2 | Inanspruchnahme der Grant-Arten durch die FördernehmerInnen}

Nachfolgend wird die Inanspruchnahme der unterschiedlichen Arten von Karriere-Grants durch die Einzelforscherlnnen auf Basis der Daten aus der Befragung der Fördernehmerlnnen dargestellt. Die Befragten selbst sind eher jünger (rd. $83 \%$ waren 40 Jahre oder jünger und rd, $26 \%$ waren 30 Jahre oder jünger) und verfügen meist erst über wenige Jahre Berufserfahrung: $83 \%$ sind 10 Jahre oder weniger, $56 \%$ sind fünf Jahre oder weniger, und $34 \%$ sind drei Jahre oder weniger berufstätig. BerufseinsteigerInnen sind allerdings nur rd. $8 \%$ der Befragten. Der überwiegende Anteil der Befragten (88\%) ist derzeit (d. h. zum Zeitpunkt der Befragung) im Bereich Forschung, Entwicklung und Innovation tätig. $34 \%$ der Befragten hatten einen Abschluss auf Master-Niveau, $66 \%$ einen Abschluss auf PhD-Niveau oder höher (Habilitation).

In den Befragungsergebnissen spiegeln sich die Anteile, was das Verhältnis Frauen zu Männern unter den Antwortenden betrifft, im Vergleich mit allen Geförderten (Grafik 56 rechts) gut wieder. Rd. $36 \%$ der Befragten sind Frauen, $63 \%$ sind Männer und $1 \%$ der Befragten wählte die Antwortoption „anderes“.

Grafik 59 zeigt die Verteilung der befragten Karriere-Grants FördernehmerInnen nach Grant-Art, hierbei sind Relocation Grants FördernehmerInnen etwas überrepräsentiert und Interview Grant FördernehmerInnen etwas unterrepräsentiert (vgl. Grafik 56 links). Dies ist insofern nachvollziehbar, da alle Geförderten seit 2011 bis 2019 befragt wurden, und es plausibel erscheint, dass sich einige der angeschriebenen FördernehmerInnen im Falle eines Interview Grants nicht mehr an die Förderung erinnern konnten ${ }^{7}$.

Grafik 59 | Verteilung der Befragten nach Grant-Art, in Prozent

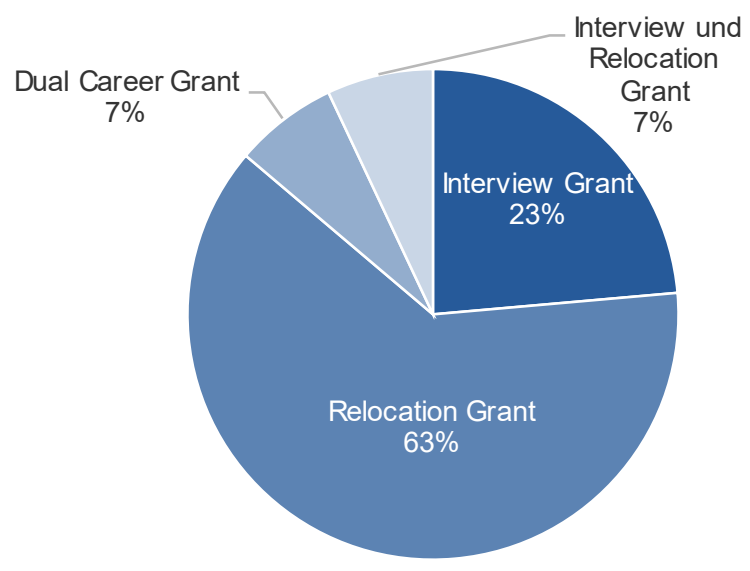

Quelle: Befragung Karriere-Grants Fördernehmerlnnen, KMU Forschung Austria; n=738

Auch die Rückmeldung einiger Befragten via E-Mail an das Evaluationsteam deuten darauf hin: Einige der angeschriebenen Personen konnten sich nicht mehr daran erinnern, im Rahmen von Karriere Grants eine Förderung erhalten zu haben. 
Interessanterweise gaben die meisten Befragten an, dass sie ausschließlich einen RelocationGrant in Anspruch genommen haben. Die Gründe, weshalb sie keinen Interview Grant vor dem Relocation Grant in Anspruch genommen haben, sind in Grafik 60 dargestellt. Der weitaus häufigste genannte Grund war demnach, dass sie den Interview Grant zu diesem Zeitpunkt nicht kannten.

Grafik 60 | Gründe, warum Befragte keinen Interview Grant vor dem Relocation Grant in Anspruch genommen haben, in Prozent

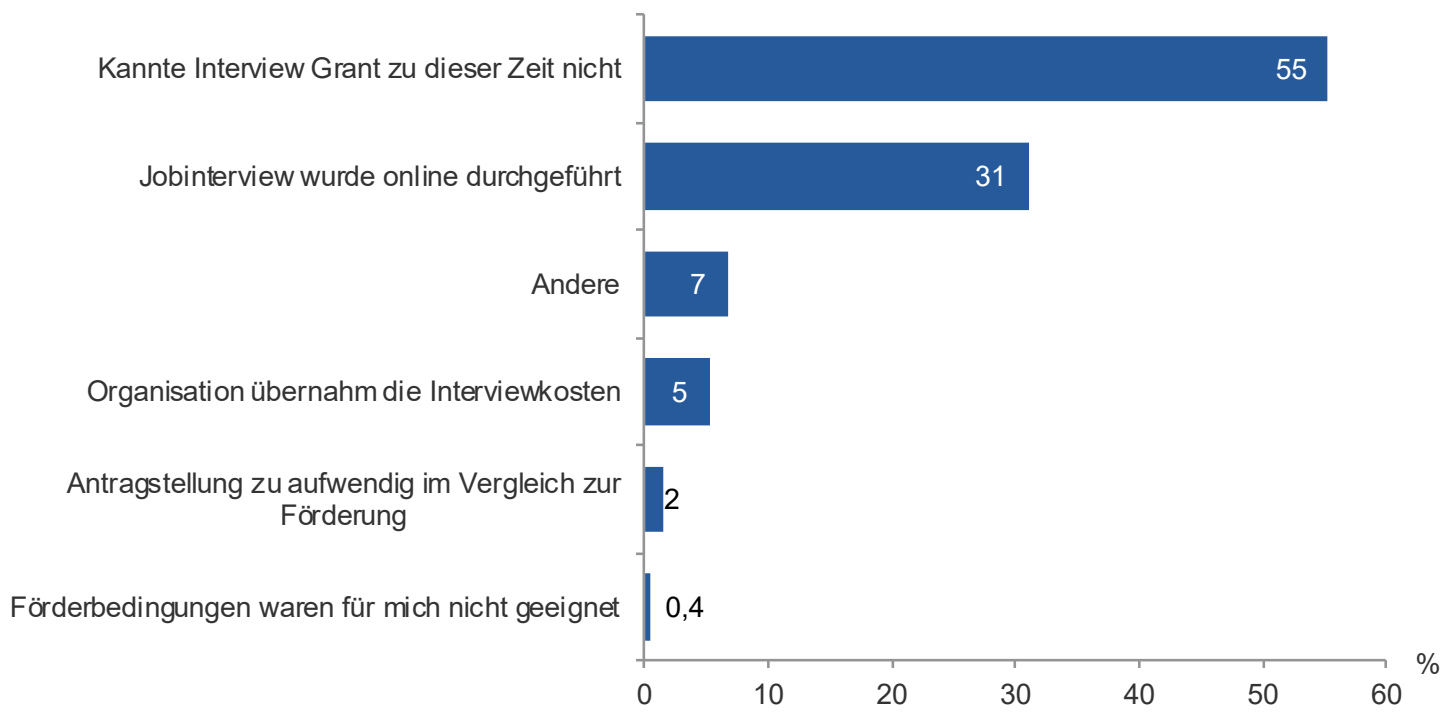

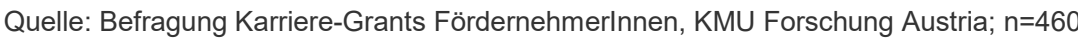

Rd. die Hälfte (51 \%) der befragten Interview Grant FördernehmerInnen gab an, dass sie von der stellenausschreibenden Organisation ein Angebot erhalten und dieses angenommen haben. Ein Anteil von $32 \%$ antwortete, dass sie kein Angebot erhalten haben, und $15 \%$ dass sie zwar ein Angebot erhalten, dieses aber nicht angenommen haben. Bei rd. $2 \%$ der Befragten war der Auswahlprozess noch im Gange. Gründe der Bewerberlnnen, eine Stelle nach dem Jobinterview doch nicht anzutreten, sind in Grafik 61 dargestellt. Erwartungsgemäß wurden häufiger Gründe genannt, die sich auf das Angebot bzw. die Organisation und das Arbeitsumfeld sowie persönliche Beziehungen zu Personen in anderen Ländern beziehen, während bestimmte Aspekte, die sich auf Österreich als Wohnort beziehen, weniger häufig als Grund genannt wurden. 
Grafik 61 | Gründe von Interview-Grant Fördernehmerlnnen, warum die angebotene Stelle in Österreich nicht angenommen wurde (Mehrfachantworten möglich)

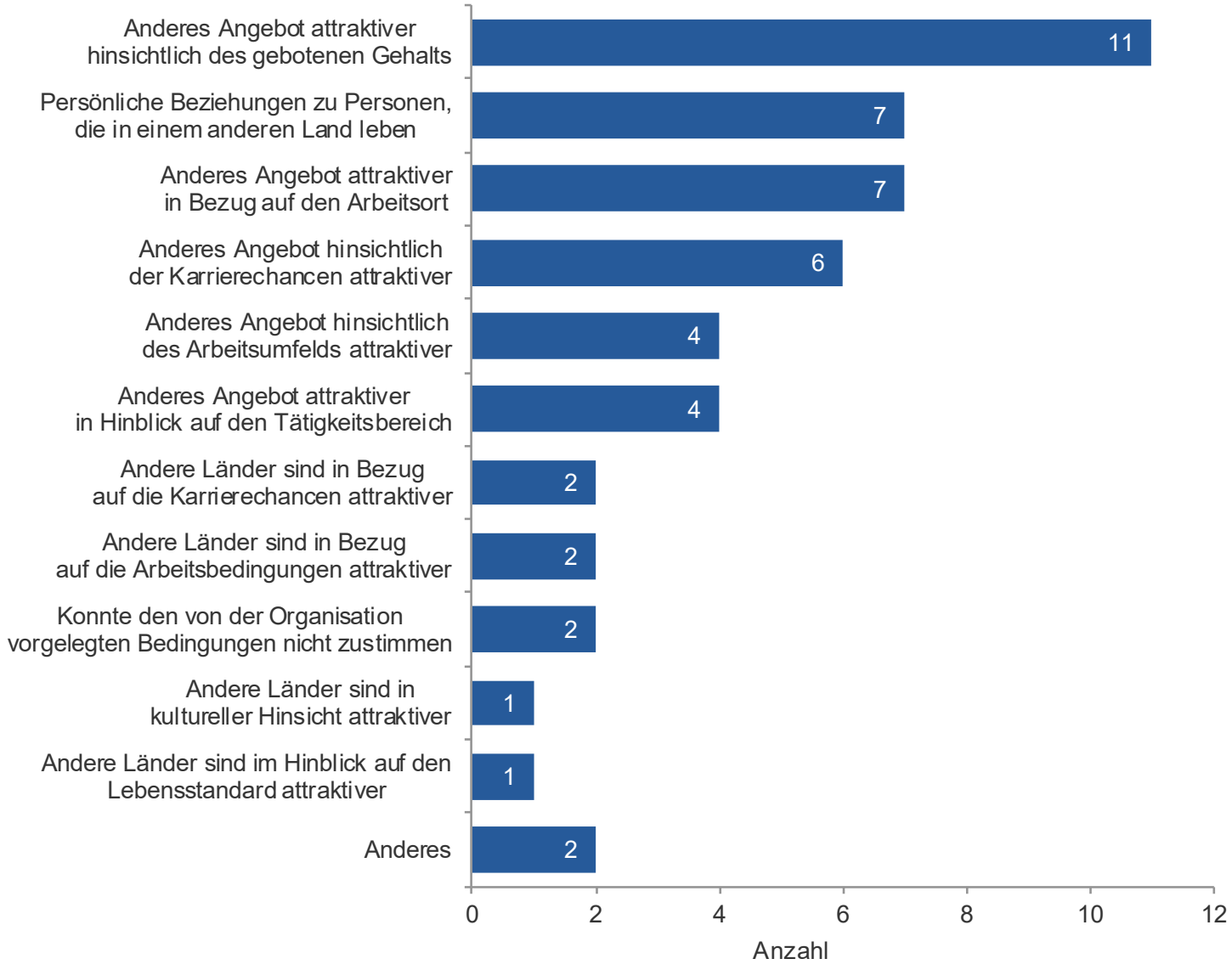

Quelle: Befragung Karriere-Grants FördernehmerInnen, KMU Forschung Austria; n=25

Interessant ist auch, dass von jenen, die einen Interview Grant beantragt haben und bereits ein Angebot von der ausschreibenden Organisation erhalten haben bzw. deren Bewerbungsprozess noch nicht abgeschlossen ist, nur $34 \%$ angaben, auch einen Relocation Grant in Anspruch nehmen zu wollen, während sich $16 \%$ noch nicht festgelegt haben und $50 \%$ der Befragten dies nicht machen wollen. Es kann allerdings nicht ausgeschlossen werden, dass diese Befragten den Relocation Grant eventuell nicht kannten.

Die befragten Interview Grant FördernehmerInnen haben neben Reisekostenzuschüssen zu einem hohen Anteil (rd. $65 \%$ ) auch Beherbergungskosten in Anspruch genommen. Aufgrund der höheren Zahl an anrechenbaren Kostenarten, ergibt sich bei den Relocation Grant FördernehmerInnen ein etwas diverseres Bild, wie in nachfolgender Grafik 62 dargestellt. 
Grafik 62 | In Anspruch genommene Kosten der Relocation Grants Fördernehmerlnnen (Mehrfachnennungen möglich)

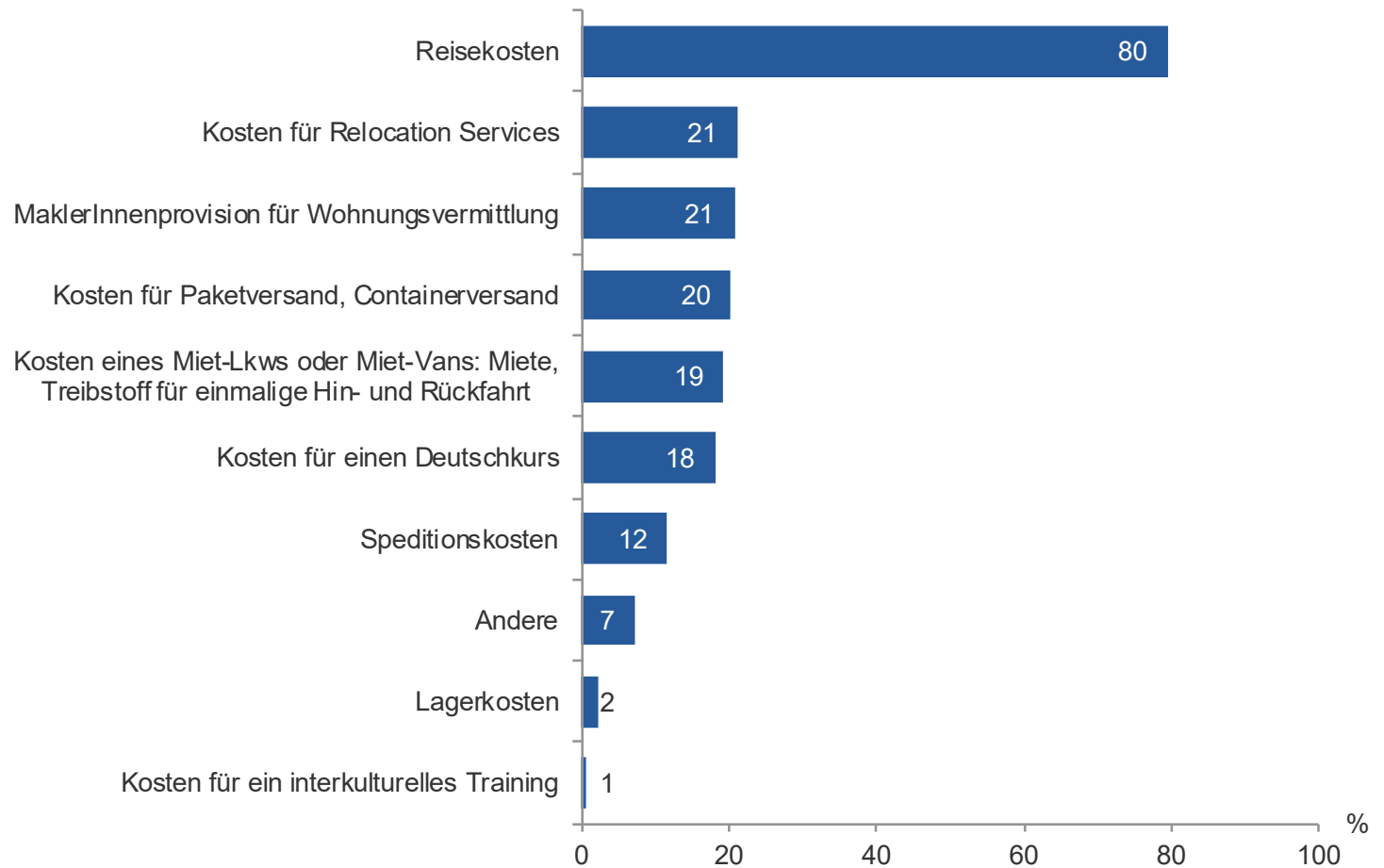

Quelle: Befragung Karriere-Grants FördernehmerInnen, KMU Forschung Austria; n=497

Dual Career Grants Fördernehmerlnnen nahmen vor allem Kosten für Deutschkurse sowie für Kinderbetreuung im Rahmen der Förderung in Anspruch.

Grafik 63 | In Anspruch genommene Kosten der Dual Career Grants FördernehmerInnen, in Prozent (Mehrfachnennungen möglich)

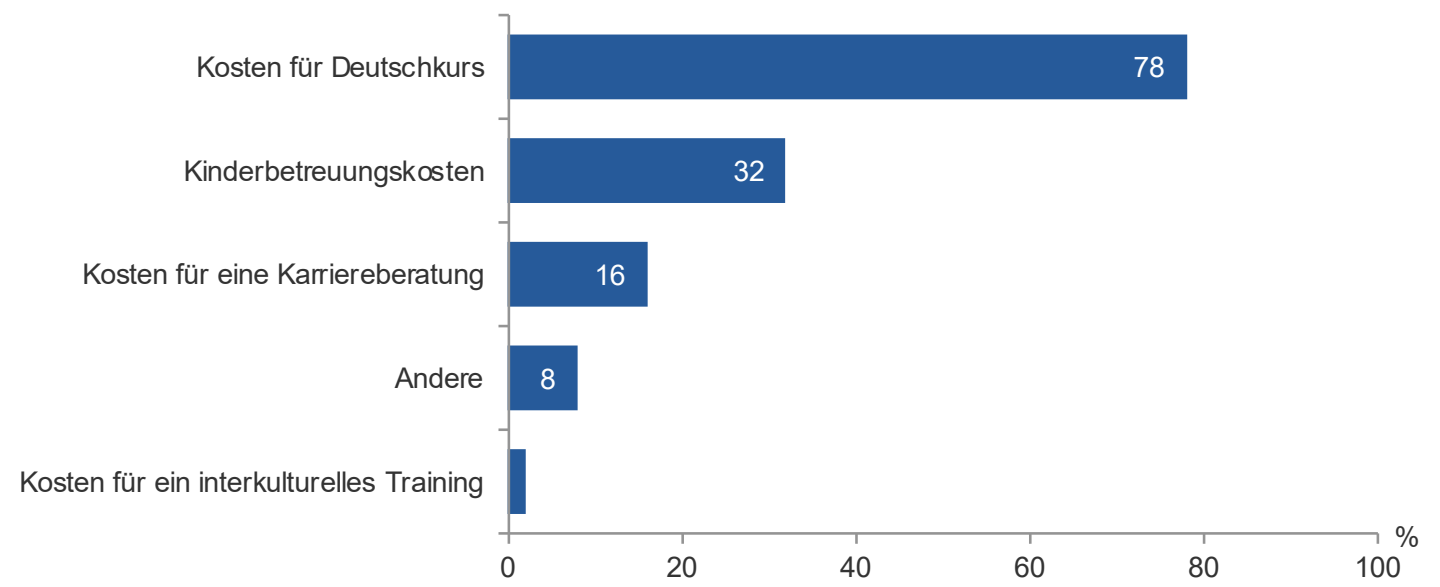

Quelle: Befragung Karriere-Grants FördernehmerInnen, KMU Forschung Austria; n=50 
FörderungsnehmerInnen eines Relocation Grants wurden befragt, ob sie alleine, mit Partnerln und/oder mit ihren Kindern nach Österreich umgezogen sind. Rd. die Hälfte der Befragten kam alleine, etwa ein Viertel mit der/dem Partnerln, $14 \%$ kamen mit Partnerln und Kindern und $2 \%$ nur mit den Kindern.

Grafik 64 | Anteil der Befragten, ob sie alleine, gemeinsam mit dem/der Partnerln und/oder ihren Kindern nach Österreich umgezogen sind, in Prozent (Mehrfachnennungen möglich)

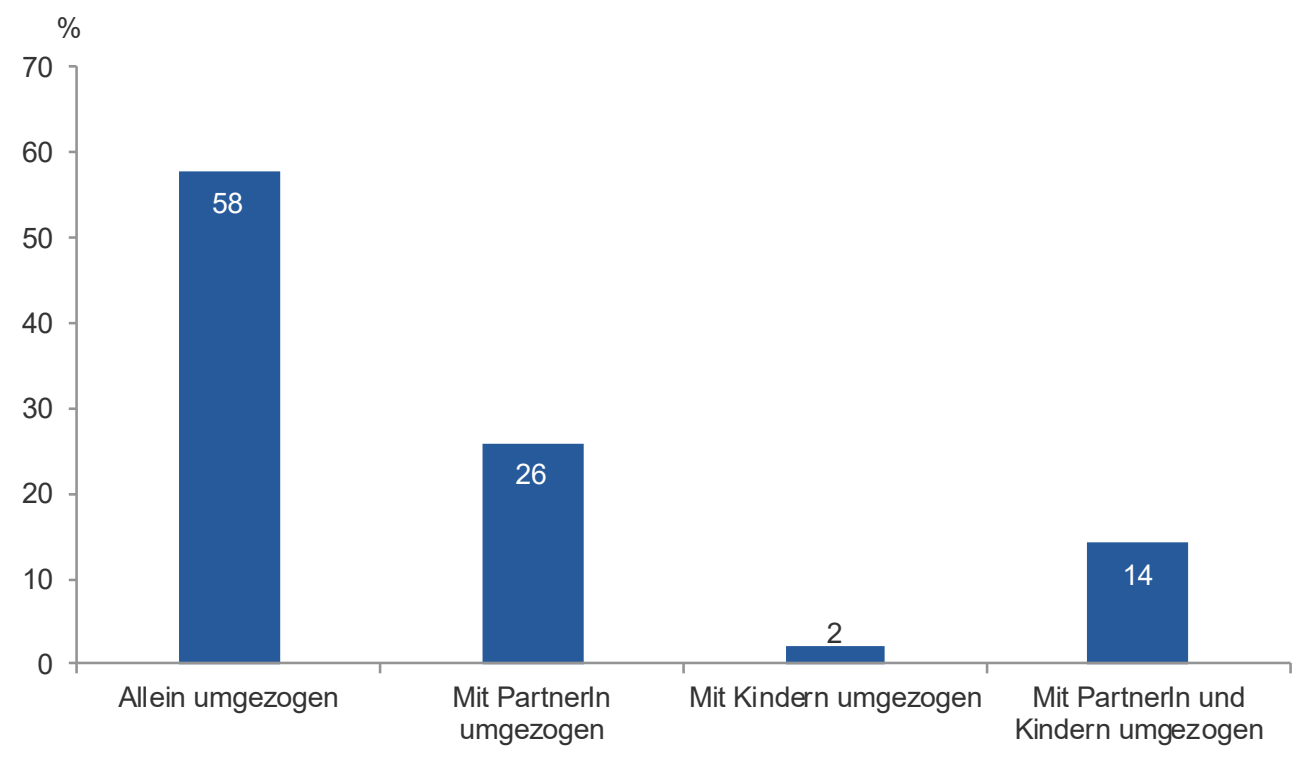

Quelle: Befragung Karriere-Grants FördernehmerInnen, KMU Forschung Austria; n=507

Erfasst wurde auch, ob die Partnerlnnen der befragten Relocation Grants Fördernehmerlnnen einen Dual Career Grant in Anspruch genommen haben bzw. planen in Anspruch zu nehmen (siehe Grafik 65 links). Dabei zeigt sich, dass nur in rd. einem Drittel der Fälle ein Dual Career Grant in Anspruch genommen wurde und nur $5 \%$ planen, dies noch zu tun. Mehr als die Hälfte der Befragten gibt an, dass die/der Partnerln keinen Dual Career Grant beantragen wird.

Grund dafür ist bei $43 \%$ die fehlende Anspruchsberechtigung, meist weil die/der Partnerln keinen Masterabschluss hat, sie/er bereits in Österreich lebt, oder sie/er ebenfalls einen Relocation Grant in Anspruch genommen hat bzw. plant in Anspruch zu nehmen. Rd. jeder Vierte gibt an, dass sie/er von der Fördermöglichkeit nichts wusste. 
Grafik 65 | Verteilung der Antworten der Relocation Grants Fördernehmerlnnen auf die Frage, ob ihr/e Partnerln einen Dual Career Grant in Anspruch genommen hat/plant in Anspruch zu nehmen und Gründe falls dies nicht der Fall war/ist, in Prozent

\section{Inanspruchnahme durch den/die Partnerln}

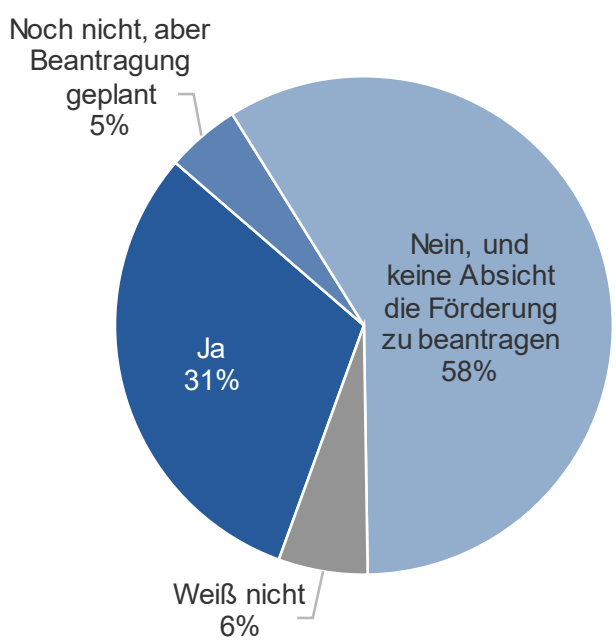

\section{Gründe, warum nicht}

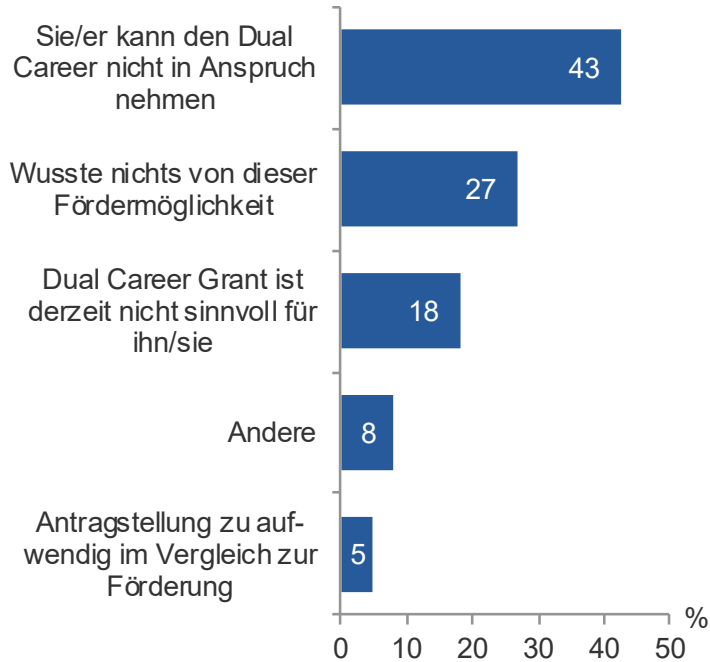

Quelle: Befragung Karriere-Grants FördernehmerInnen, KMU Forschung Austria; n=208 (links) und n=122 (rechts)

Ein Anteil von 79 \% der Befragten, welche nach Österreich umgezogen sind, antworteten, dass sie immer noch in Österreich leben bzw. arbeiten. Die häufigsten Gründe des Wegzugs von jenen, die nicht mehr in Österreich leben, waren, dass sie einen Job in einem anderen Land bekommen haben und dass ihr Beschäftigungsvertrag endete. Als gegenwärtigen Aufenthaltsort gaben diejenigen, die nicht mehr in Österreich wohnen, am häufigsten ein anderes EU Land (37\%, außer DACH) und die DACH-Region (34 \%) an.

\subsection{1 | Wirkungen und Bewertung der Karriere-Grants}

Die Karriere-Grants Förderung spielt bei der Entscheidung, ob jemand nach Österreich kommt nur für einen sehr geringen Anteil der Befragten eine entscheidende Rolle, wie die Antworten der Befragten in den nachfolgenden Grafiken zeigen.

Am ehesten zeigen noch die Interview Grants Wirkung. So gaben etwa rd. ein Viertel (23\%) der Befragten an, sie wären ohne Interview Grant nicht oder wahrscheinlich nicht für das Jobinterview nach Österreich gekommen. Von dieser Gruppe hätten allerdings rd. $36 \%$ das Interview online abgehalten. In der Gruppe der Befragten, die ohne Förderung nicht gekommen wären, befinden sich signifikant häufiger Personen aus dem außereuropäischen Ausland, d. h. vor allem Personen außerhalb Europas wären ohne Förderung häufiger als Personen innerhalb Europas nicht 
nach Österreich für ein Jobinterview gekommen. Dennoch wären insgesamt drei Viertel der FördernehmerInnen von Interview Grants auch ohne Förderung zum Jobinterview nach Österreich gekommen.

Grafik 66 | Wirkungen des Interview Grants auf die Entscheidung, für ein Job Interview nach Österreich zu kommen, in Prozent

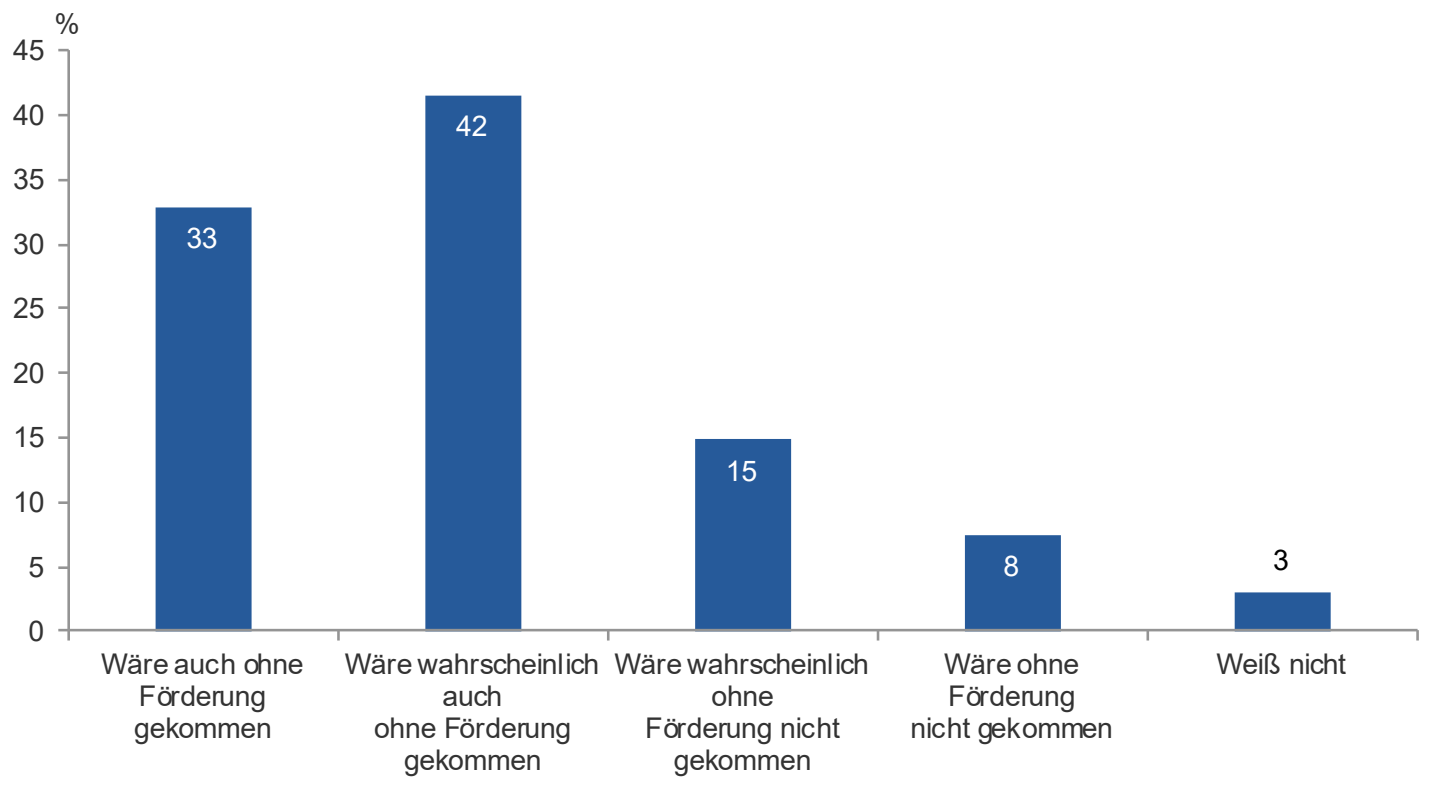

Quelle: Befragung Karriere-Grants Fördernehmerlnnen, KMU Forschung Austria; n=161

Das Antreten einer Stelle in Österreich wird so gut wie gar nicht von den Relocation Grants beeinflusst, für mehr als $90 \%$ der Befragten ist dies nicht der Fall. Nur für einige wenige Hochqualifizierte war der Relocation Grant ein entscheidender Grund, nach Österreich zu kommen. Rd. $5 \%$ der Befragten antworteten, dass sie ohne den Relocation Grant nicht oder wahrscheinlich nicht nach Österreich gekommen wären. Ähnlich wie beim Interview Grant, sind es häufiger ForscherInnen außerhalb Europas, die angeben, dass sie ohne die Förderung nicht nach Österreich gekommen wären. 
Grafik 67 | Wirkungen des Relocation Grants auf die Entscheidung, aus beruflichen Gründen nach Österreich umzuziehen, in Prozent

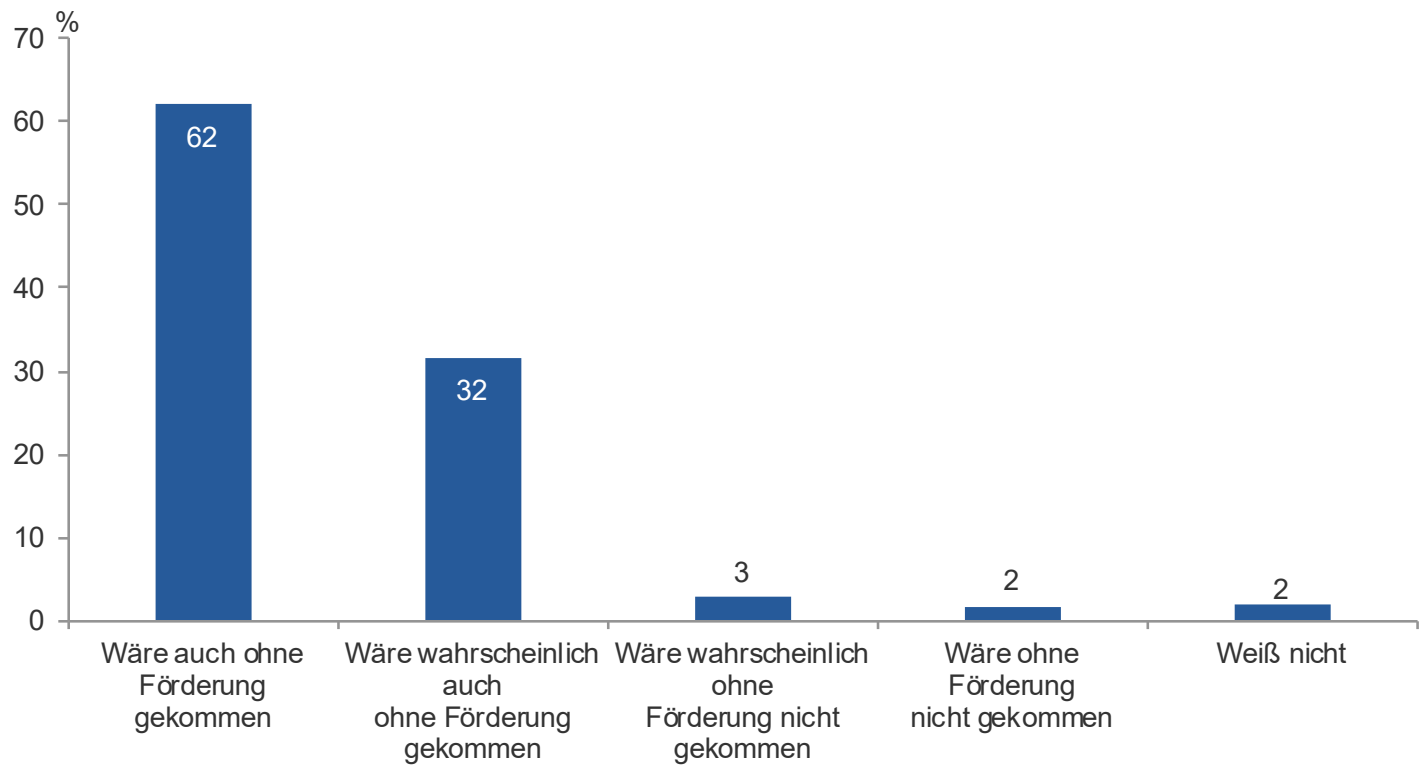

Rundungsdifferenzen nicht ausgeglichen

Quelle: Befragung Karriere-Grants Fördernehmerlnnen, KMU Forschung Austria; n=498

Beim Dual Career Grant kann die Förderung naturgemäß keinen Einfluss auf die Bereitschaft haben, nach Österreich umzuziehen, da es sich um die Partnerin bzw. den Partner handelt, der bereits einen Relocation Grant in Anspruch genommen hat. So antwortet auch niemand, sie/er wäre ohne Förderung nicht nach Österreich umgezogen.

Insgesamt muss festgehalten werden, dass die Karriere-Grants kaum eine Auswirkung auf die Entscheidung von EinzelforscherInnen haben, ob sie aus beruflichen Gründen nach Österreich umziehen. In nur (sehr) wenigen Fällen dürften die Karriere-Grants dazu beigetragen haben, dass Hochqualifizierte für ein Jobinterview nach Österreich kommen bzw. hier eine Stelle antreten.

Die Fördernehmerlnnen bewerten die Programmlinie sehr positiv, was sich auch am hohen Anteil an Weiterempfehlungen zeigt. Die große Mehrheit der Befragten hat bzw. wird Karriere-Grants weiterempfehlen, wie Grafik 68 zeigt. 
Grafik 68 | Weiterempfehlung von Karriere Grants an Kolleginnen und Kollegen, in Prozent

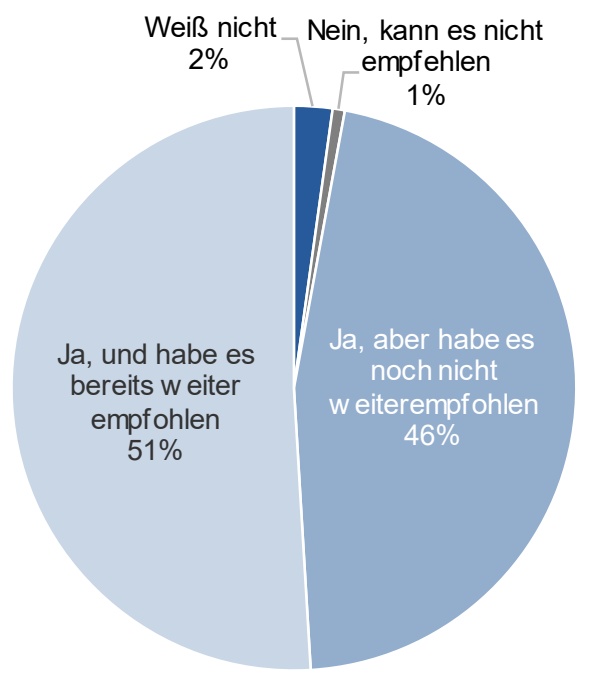

Quelle: Befragung Karriere-Grants FördernehmerInnen, KMU Forschung Austria; n=728

Aus Sicht der Befragten gibt es unterschiedliche Verbesserungsvorschläge. Allgemeine Verbesserungsvorschläge der Befragten zur Karriere-Grants Programmlinie beziehen sich am häufigsten auf die Bewerbung der Programmlinie und die Verbreitung von Informationen über KarriereGrants, in erster Linie bei den stellenausschreibenden Organisationen, aber auch bei für Einreisende wichtige Kontaktstellen wie beispielsweise österreichischen Botschaften (bzw. deren Website) und soziale Medien (z. B. Linkedln). Generell, aber vor allem bei den Relocation Grants bestanden auch Unsicherheiten bzgl. der anrechenbaren Kosten. Öfters genannt wurde beispielsweise, dass nicht bekannt war, dass auch Visa-Kosten anrechenbar sind. Bei den Dual Career FördernehmerInnen wurde vereinzelt der Wunsch geäußert, auch Reise- und Umzugskosten der Partnerin / des Partners als Kosten anrechnen zu können. Was die Förderhöhe betrifft, gaben einige Befragte aus weiter entfernten Gebieten (Asien, Amerika, Ozeanien) an, dass die Förderung nur einen Bruchteil der tatsächlichen Umzugskosten abdeckt, vor allem wenn eine Forscherin bzw. ein Forscher mitsamt der Familie umzieht. 


\section{7 | Die österreichische Jobbörse für Forschung, Ent- wicklung und Innovation}

Die FFG Jobbörse ist ein frei und kostenlos zugängliches Online-Service, das in Österreich verfügbare Stellen in Forschung, Entwicklung und Innovation listet. Die Jobbörse gibt es seit 2011 und dient dazu, StellenanbieterInnen und Stellensuchende im Bereich Forschung, Technologie und Innovation zusammenzubringen. Sie listet sowohl Jobs als auch Praktikums-, Masterarbeitsund Dissertationsstellen. Grundlage der Datenbank bilden die veröffentlichten Ausschreibungen von Universitäten, Fachhochschulen und außeruniversitären Forschungseinrichtungen, welche automatisch in die Jobbörse eingespielt werden. Ergänzt wird dies durch eine Suche nach Ausschreibungen auf den Websites registrierter Unternehmen ${ }^{8}$. Zusätzlich werden auch Jobbörsen (z. B. karriere.at) und Websites von Personaldienstleistern (z. B. hays.at) nach relevanten Stellenausschreibungen durchsucht. Es werden nur Stellen gelistet, die bestimmten Kriterien entsprechen: Die Stelle muss aktuell verfügbar sein, der Dienststandort muss in Österreich sein, und die Stelle muss einen Bezug zu Forschung, Entwicklung und Innovation haben. Gelistet werden Stellen, die direkt in der Forschung angesiedelt sind, und Stellen im Umfeld von F\&E (z. B. im Technologietransfer, Projektmanagement oder der Forschungsförderung $)^{9}$. Die Jobbörse beinhaltet sowohl deutsch- als auch englischsprachige Ausschreibungen.

Für den Zeitraum 1. Quartal 2013 bis 1.Quartal 2019, für den vergleichbare Daten vorliegen (Ausnahme: 3.Quartal 2016), zeigt sich eine Zunahme der gelisteten Jobs von 1.281 (1.Quartal 2013) auf 2.331 (1.Quartal 2019). Die Anzahl der Websiteaufrufe der Jobbörse variiert relativ stark, tendenziell haben die Seitenaufrufe aber eher abgenommen. Dasselbe lässt sich für die Zahl der WebsitebesucherInnen (Unique Visitors) beobachten, auch hier nahm die Anzahl tendenziell vor allem seit 2016 eher ab. Die Entwicklung seit 2019 ist schwierig einzuschätzen, da es im 2. Quartal 2019 zu einer Umstellung der Erhebungsmethode kam, die zu einer geringeren Zahl erfasster Unique Visitors (ca. -40 \%) führte. Auf Basis der Angaben der FFG wurde die Anzahl der Unique Visitors daher geschätzt, um sie für den gesamten Zeitraum 2013 bis 2019 besser vergleichbar machen zu können. Im Zeitraum von 2013 bis 2018 wurden pro Quartal durchschnittlich 5.220 Unique Visitors bzw. für 2013 bis 2019 unter Einbezug der neu berechneten Werte 5.313 Unique Visitors gezählt (das 3. Quartal 2016 blieb hierbei unberücksichtigt). Im 4. Quartal 2019 wurde der Zielwert von 6.000 BesucherInnen (geschätzter Wert) erreicht.

\footnotetext{
8 Zum Startzeitpunkt der Jobbörse wurde von der FFG gemeinsam mit dem damaligen Crawling-Anbieter ein Grundstock von ca. 500 Websites erstellt. Dieser Grundstock wurde seither durch Websites von Unternehmen ergänzt, die sich für die Jobbörse registriert haben.

${ }^{9}$ Quelle: https://www.ffg.at/jobboerse/faq , abgerufen am 14.10.2020
} 
Grafik 69 | Entwicklung der Anzahl veröffentlichter Jobs, der Websiteaufrufe (page impressions) und der WebsitebesucherInnen (unique visitors) der FFG Jobbörse, 2013-2019

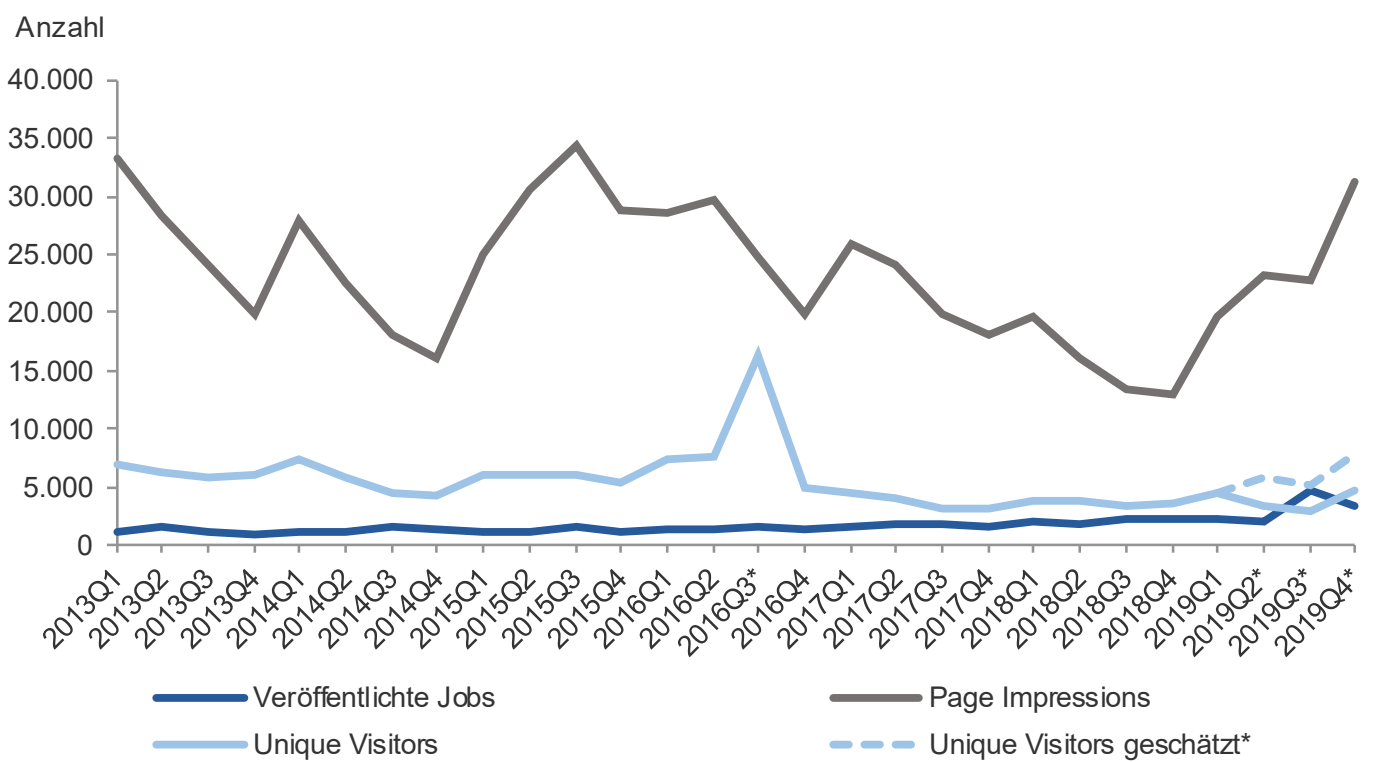

*Das 3. Quartal 2016 ist aufgrund einer anderen Zählmethode nicht mit den anderen Quartalen vergleichbar. Für das 2. das 3. und 4. Quartal 2019 wurden auf Basis der Angaben der FFG zwecks Vergleichbarkeit neue Werte für die Unique Visitors geschätzt.

Quelle: FFG; Darstellung und Berechnung KMU Forschung Austria

Die meisten ausgeschriebenen Stellen zwischen 2011 und 2019 entfielen auf das Bundesland Wien, dahinter folgt die Steiermark und Oberösterreich. Für Wien liegen die jeweiligen Anteile gemessen an allen veröffentlichten Jobs in Österreich innerhalb eines Quartals zwischen $29 \%$ (4. Quartal 2015) und $42 \%$ (4.Quartal 2018), für die Steiermark zwischen rd. $17 \%$ (4.Quartal 2013) und 32 \% (2.Quartal 2016) und für Oberösterreich zwischen 8 \% (4. Quartal 2013) und $16 \%$ (3.Quartal 2017). Die Anteile der Stellenausschreibungen, die auf die Bundesländer Niederösterreich, Salzburg, Tirol, Kärnten und Vorarlberg entfallen, befinden sich im einstelligen bzw. niedrigen zweistelligen Bereich, wenn man sie im Zeitverlauf von 2011 bis 2019 betrachtet. Das Burgenland weist nur einen sehr geringen Anteil an Jobveröffentlichungen auf, in jedem Quartal liegt dieser Wert unter $1 \%$ aller Veröffentlichungen. Es zeigen sich keine größeren Verschiebungen oder Änderungen in den Anteilen, die auf das jeweilige Bundesland entfallen. Auch wenn sich teils größere Schwankungen im Zeitverlauf zeigen, in jedem Quartal entfällt mehr als die Hälfte der Jobveröffentlichungen auf die beiden Bundesländer Steiermark und Wien.

Der überwiegende Anteil der in der Jobbörse gelisteten Veröffentlichung sind F\&E-Jobs, Ausschreibungen für Abschlussarbeiten (max. $20 \%$ ) und Praktika (max. $9 \%$ ) finden sich deutlich weniger, wie in Grafik $70^{10}$ dargestellt.

\footnotetext{
${ }^{10}$ Vergleichbare Daten sind erst für den Zeitraum ab dem 4.Quartal 2013 verfügbar.
} 
Grafik 70 | Verteilung der Art der Veröffentlichungen in der Jobbörse, nach Quartal, 3. Quartal 2014 bis 4. Quartal 2019

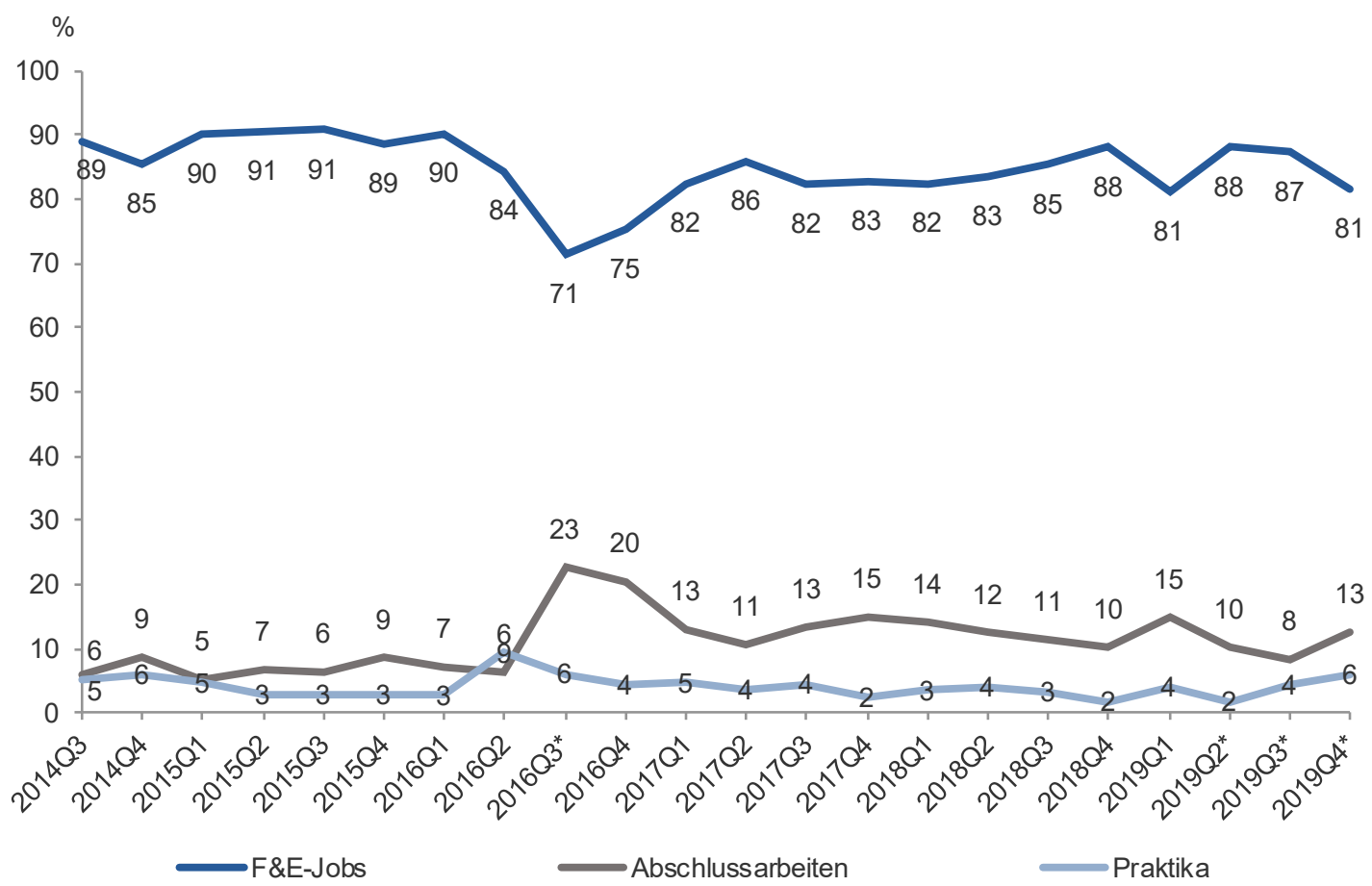

*Das 3. Quartal 2016 ist aufgrund einer anderen Zählmethode nicht mit den anderen Quartalen vergleichbar. Ab dem 2. Quartal 2019 erfolgte eine Umstellung der Erhebungsmethode.

Quelle: FFG, Darstellung KMU Forschung Austria

Was die ausschreibenden Organisationen betrifft, liegen für einen Vergleich nach Organisationstyp nur vergleichbare Daten für den Zeitraum von 01.07.2019 bis 24.06.2020 vor. Die in Grafik 71 dargestellten Werte sind daher als Momentaufnahme für diesen Zeitraum zu betrachten. Es zeigt sich, dass die Stellen in Unternehmen nicht nur die am häufigsten gelisteten sind, sondern auch deutlich über jenen an Universtäten, außeruniversitären Forschungseinrichtungen und Fachhochschulen insgesamt liegen. 
Grafik 71 | Anzahl der Veröffentlichungen in der Jobbörse nach Organisationstyp, 01.07.2019 bis 24.06.2020

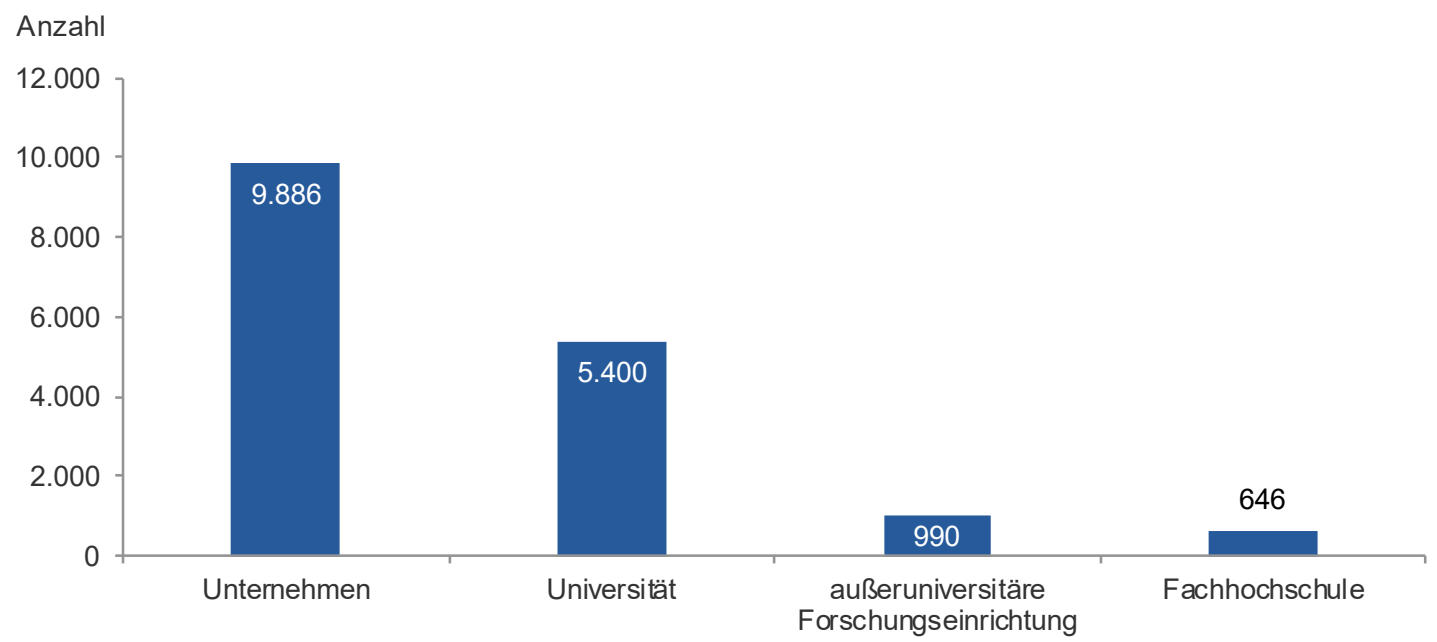

Quelle: FFG, Darstellung KMU Forschung Austria

Auch für das in den Veröffentlichungen genannte Zeitausmaß der Stellen (Voll- oder Teilzeit) und die Sprache der Stellenausschreibung liegen nur für den Zeitraum 01.07.2019 bis 09.07.2020 Zahlen vor, wobei beachtet werden muss, dass bei der Zählung des Zeitausmaßes Ausschreibungen zweifach gezählt werden können, wenn in der Ausschreibung sowohl Voll- als auch Teilzeit als Option angegeben sind. So gab es im genannten Zeitraum rd. 15.000 Veröffentlichungen, in denen eine Vollzeitstelle ausgeschrieben war, und rd. 3.300 Veröffentlichungen, in denen eine Teilzeitstelle ausgeschrieben war. Rd. 13.800 Ausschreibungen waren in deutscher Sprache, und rd. 3.800 Ausschreibungen in englischer Sprache verfasst.

Betrachtet man die Wissenschaftsdisziplinen, für die Veröffentlichungen in der Jobbörse zu finden sind, zeigt sich folgendes Bild: Der überwiegende Anteil der Veröffentlichungen kann den technischen Wissenschaften zugeordnet werden. Auf die Naturwissenschaften entfallen je nach Quartal zwischen $15 \%$ und $27 \%$, auf die Sozial- und Wirtschaftswissenschaften $5 \%$ bis $10 \%$, auf die Medizin $2 \%$ bis $8 \%$ und auf die Geistes- und Kulturwissenschaften $1 \%$ bis $5 \%$. 
Grafik 72 | Verteilung der Veröffentlichungen in der Jobbörse nach Wissenschaftsdisziplin, in Prozent der Stellenausschreibungen

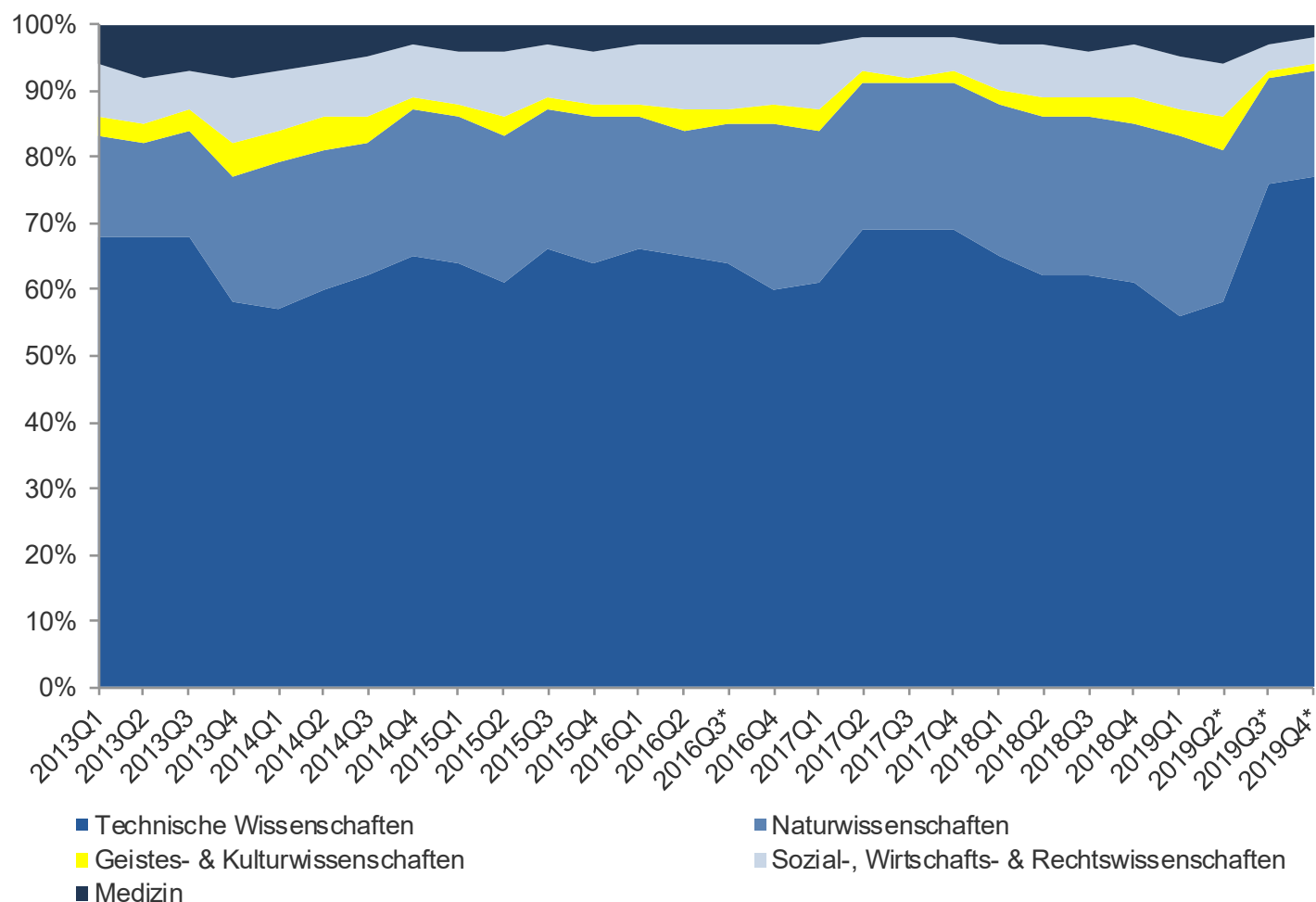

*Das 3. Quartal 2016 ist aufgrund einer anderen Zählmethode nicht mit den anderen Quartalen vergleichbar. Ab dem 2. Quartal 2019 erfolgte eine Umstellung der Erhebungsmethode.

Quelle: FFG, Darstellung KMU Forschung Austria

Abschließend ist festzuhalten, dass der angestrebte Zielwert an Besucherlnnen nicht erreicht werden konnte, die Zunahme an Ausschreibungen im Beobachtungszeitraum aber auf eine gestiegene Nutzung durch Organisationen hindeutet. Ein weiteres Kriterium, um die Jobbörse zu beurteilen, ist der Grad der Abdeckung aller veröffentlichten Stellen in diesem Bereich in Österreich und ihre Reichweite. Da die Jobbörse neben den Websites der Organisationen auch frei zugängliche Jobportale durchsucht, kann von einer guten Abdeckung ausgegangen werden. Es kann aber weder die Abdeckung noch die Reichweite der Jobbörse abschließend bewertet werden, da hierzu Vergleichsdaten fehlen. Zudem kann auf Basis der verfügbaren Informationen nicht geklärt werden, ob die Jobbörse sowohl für Suchende als auch Jobanbieterlnnen einen Zusatznutzen gegenüber anderen Dienstleistungsangeboten bringt. 


\section{8 | Begleitmaßnahmen}

Im Rahmen von Talente wurden vier Begleitmaßnahmen umgesetzt: Prämierungsveranstaltungen in der Programmlinie Praktika für Schülerinnen und Schüler, Netzwerkveranstaltungen und eine Infoveranstaltung im Rahmen von Talente regional, Infoveranstaltungen für FEMtech Forschungsprojekte und FEMtech Netzwerktreffen.

Im Rahmen der SchülerInnenpraktika finden jährlich Prämierungsveranstaltungen der 20 Schülerlnnen mit den besten Praktikum-Reports (entspricht ca. $10 \%$ der eingereichten Reports) statt. Von 2011 bis zur ersten Hälfte 2020 fanden somit insgesamt 10 Prämierungsveranstaltungen statt, acht davon in der Urania Wien, eine in der Sky Stage TechGate Vienna und eine im Haus der Forschung in Wien. Zu den Prämierungsveranstaltungen wurden PraktikantInnen, Begleitpersonen und BetreuerInnen eingeladen. Es meldeten sich im Durchschnitt 61 Teilnehmerlnnen an, von sieben Prämierungsveranstaltungen gibt es Daten zur Teilnehmerlnnenzahl, diese liegt durchschnittlich bei ca. 58 TeilnehmerInnen. Es zeigt sich, dass nicht nur die prämierten SchülerInnen Interesse an der Veranstaltung zeigen, sondern auch ihr Umfeld bzw. die FördernehmerInnen. Die Prämierungsveranstaltungen dienen nicht der Kundlnnenakquise bzw. -beratung, sondern haben zum Ziel, engagierte Schülerlnnen vor den Vorhang zu holen. Dies kann mit einer derartigen Veranstaltung erreicht werden, zumal die Fotos der Prämierungsfeier als auch die Namen der prämierten SchülerInnen sowie der Organisationen, in der diese Praktika absolviert wurden, online auf der Website der FFG einsehbar sind. Es stellt sich die Frage, ob diese Prämierungen bzw. deren Fotos auch Grundlage von weiteren Promotionsaktivitäten bei der direkten Ansprache von Unternehmen sein könnten, da Unternehmen dadurch auch Öffentlichkeitswirkung erlangen.

Im Rahmen von Talente regional wurden insgesamt sechs Netzwerkveranstaltungen und eine Infoveranstaltung abgehalten, die sechs Netzwerkveranstaltungen in der Urania Wien, die Infoveranstaltung im Haus der Forschung in Wien. Für diese Veranstaltungen meldeten sich im Durchschnitt ca. 85 Personen an. Für fünf Veranstaltungen liegen auch Werte über die ungefähre Anzahl an TeilnehmerInnen vor: im Durchschnitt nahmen rd. 71 Personen teil. Im Rahmen dieser Veranstaltungen wurde zum einen die jeweils aktuelle Ausschreibung von Talente regional vorgestellt, als auch zu bestimmten Schwerpunktthemen des BMK und darüberhinausgehend (Impuls-)Vorträge gehalten, die Möglichkeit zum Erfahrungsaustausch geboten sowie 2011 und 2019 Best Practice Beispiele präsentiert und 2011 ein World Café veranstaltet. Aufgrund des Vergleichs der Anzahl der Teilnehmenden und der Einreichungen bei Talente regional kann von einer guten Abdeckung bei den InteressentInnen ausgegangen werden. Da es sich bei den einreichenden Organisationen großteils um keine typische FFG-Klientel handelt sowie die Projektanforderungen als herausfordernd beschrieben werden, kommt aus Sicht der EvaluatorInnen der Präsentation von Good Practice Beispielen und der Möglichkeit des Erfahrungsaustauschs im Rahmen solcher Informationsveranstaltungen große Bedeutung zu. 
Für die Programmlinie FEMtech Forschungsprojekte wurden bis einschließlich $2019^{11}$ zwei Infoveranstaltungen (2017 und 2018) im Haus der Forschung abgehalten. Im Rahmen dieser Veranstaltungen wurden die jeweils aktuelle Ausschreibung sowie einige FEMtech Forschungsprojekte von den Fördernehmerlnnen vorgestellt und den Teilnehmenden die Möglichkeit gegeben, Fragen zu stellen. Im Jahr 2017 nahmen ungefähr 100 Personen, im Jahr 2018 ca. 70 Personen an der Veranstaltung teil. Wenn man diese Zahlen der Anzahl der Einreichungen gegenüberstellt (2017: 54, 2018: 41), so kann davon ausgegangen werden, dass die Infoveranstaltungen von den InteressentInnen gut besucht werden. Besonders zielführend scheint die Präsentation von bereits geförderten FEMtech Forschungsprojekten, da diese zeigen können, was es in der Praxis bedeutet, den Genderfokus in den Mittelpunkt der Forschung zu rücken und welcher Mehrwert sich daraus ergibt. Dadurch können die Infoveranstaltungen auch für bereits bestehende FFG-Klientel einen großen Nutzen haben, was hier von besonderer Relevanz ist.

Von 2011 bis 2019 wurden insgesamt 18 FEMtech Netzwerktreffen abgehalten, davon 10 in Wien, und jeweils eines in Klagenfurt, Wiener Neustadt, Innsbruck, Eisenstadt, Salzburg, Graz, Linz und Dornbirn. Durchschnittlich meldeten sich 98 Personen für die Netzwerktreffen an. Zahlen zu den Teilnehmerlnnen stehen nur für 10 Netzwerktreffen zur Verfügung, die durchschnittliche Teilnehmerlnnenzahl betrug hier 58 Personen, wobei die Netzwerktreffen in Wien deutlich mehr Personen besuchten als in den Bundesländern ( $r d .75$ vs. rd. 40) ${ }^{12}$. Neben Impulsvorträgen und Podiumsdiskussionen gibt es Raum für den informellen Austausch. Die Präsentationen bzw. Zusammenfassungen der Diskussionen in den Netzwerktreffen können online über die FEMtech Website (https://www.femtech.at/) $)^{13}$ abgerufen werden. Die Netzwerktreffen decken ein breites und relevantes Themenfeld ab und reichen von der Thematisierung von Diversität und ihrem Nutzen (z. B. "Chancengleichheit schafft Wettbewerbsvorteile“, „Nutzen durch Vielfalt") über Work-Life-Balance (z. B. „Führung in Teilzeit“, „Väterkarenz“), Karriereentwicklung („Bilder im Kopf - Karrieren - Arbeitswelt“, „Mentoring - Bewährtes neu gedacht“) über die Auswirkungen allgemeiner Trends auf die Chancengleichheit (z. B. „Digitalisierung macht alles anders. Auch die Chancengleichheit"). Zielgruppe sind Personen, die die Rahmenbedingungen für Frauen im naturwissenschaftlich-technischen Bereich verbessern wollen, und es nehmen auch vorwiegend Personen an den Treffen teil, die sich mit Chancengleichheit in Forschung, Naturwissenschaft und Technik beschäftigen. Dem Charakter eines Netzwerktreffens für themenaffine Personen entsprechend wird das Netzwerken innerhalb der mit dem Thema befassten Personen unterstützt. Für eine breite Wirkung in die Forschungscommunity müssten zusätzliche Maßnahmen (z. B. bei Veranstaltungen im restlichen FFG-Portfolio) umgesetzt werden. Am Infoschalter der FFG gibt es die Möglichkeit der Beratung zum Förderschwerpunkt Talente, die KundInnenakquise steht aber bei der derzeitigen Ausgestaltung der Netzwerktreffen nicht im Vordergrund.

\footnotetext{
${ }^{11}$ Die Infoveranstaltung zur 7. Ausschreibung in 2020 wurde aufgrund der Corona-Maßnahmen erstmals online abgehalten.

${ }^{12}$ Die genaue Teilnehmerlnnenzahl war nicht zu ermitteln, da es sich um frei zugängliche Veranstaltung handelte.

${ }^{13}$ FEMtech ist eine Initiative des BMK für Aktivitäten zur Bewusstseinsbildung, zur Sensibilisierung, zur Sichtbarmachung sowie zur Förderung der Karrieren von Frauen in Forschung und Technologie. Sie steht in keinem direkten Zusammenhang mit den Programmlinien FEMtech im Programm Talente.
} 


\section{7 | Gesamtbetrachtung des Förderschwer- punkts}

\subsection{Gesamtstruktur}

Folgende Grafik zeigt die Verteilung der geförderten Organisationen / Einzelfoscherlnnen sowie die Verteilung der Projektbeteiligungen der Organisationen / EinzelforscherInnen in Talente von 2011 bis Juni 2020 anhand der Klassifikation in den FFG Daten ${ }^{14}$. Ein gutes Drittel der FördernehmerInnen sind Unternehmen (36\%), jede Zehnte eine Hochschule und $4 \%$ außeruniversitäre Forschungseinrichtungen. Der hohe Anteil beim geförderten Organisationstyp Sonstige (49\%) ist auf die hohe Anzahl an Einzelforscherlnnen in der Programmlinie Karriere-Grants zurückzuführen.

Hingegen werden EinzelforscherInnen nur einmal bzw. zweimal im Rahmen der Programmlinie Karriere-Grants gefördert, was sich im geringeren Anteil bei den Projektbeteiligungen niederschlägt (17\%). Außeruniversitäre Forschungseinrichtungen (27\%) und Hochschulen (18\%) haben hier vergleichsweise mehr Projektbeteiligungen, da diese aufgrund ihrer Struktur (Expertise in einer oder mehreren Wissenschaftsdisziplinen) und ihres Fokus auf Forschung, Entwicklung und Innovation in der Regel häufiger an geförderten Projekten teilnehmen als Unternehmen.

Grafik 73 | Verteilung der geförderten Organisationen/EinzelforscherInnen und Projektbeteiligungen in Talente, nach Organisationstyp in Prozent

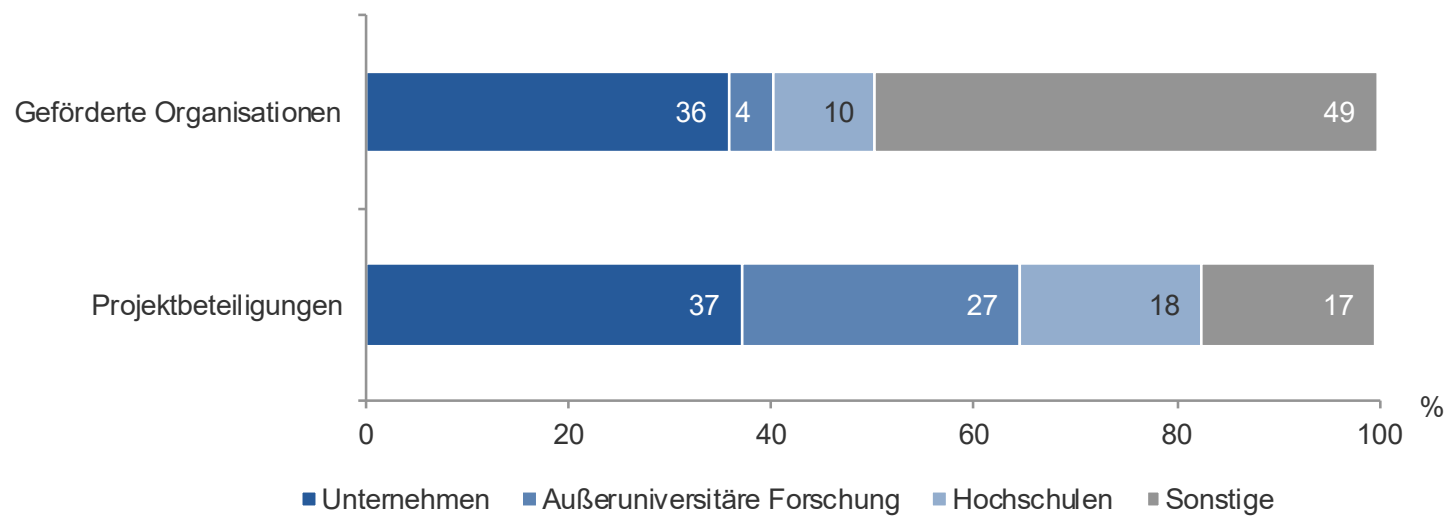

Quelle: FFG, Berechnung und Darstellung KMU Forschung Austria; Geförderte Organisationen/Einzelforscherlnnen:

\footnotetext{
${ }^{14}$ Die FFG klassifiziert Organisationen nach den Kategorien: Forschungseinrichtungen (das sind in erster Linie außeruniversitäre Forschungseinrichtungen), Hochschulen (Universitäten und Fachhochschulen), Intermediäre, Sonstige (Gebietskörperschaften und Non-Profit-Organisationen, aber auch EinzelforscherInnen) und Unternehmen. Es ist zu beachten, dass universitäre Hochschulinstitute als eigene Organisationseinheit gezählt werden, weshalb auf Hochschulen höhere Anteile entfallen, als wenn man die Hochschule als Organisationseinheit definieren würde.
} 
Um eine Einschätzung über die finanziellen Anteile der jeweiligen Programmlinien an Talente zu erhalten, wurden alle genehmigten Förderbarwerte in den Programmlinien von 2011 bis 2019 aufsummiert und ins Verhältnis zu den gesamten genehmigten Förderbarwerten in Talente gesetzt. Das Ergebnis hierzu ist in Grafik 74 dargestellt. Insgesamt wurden im Zeitraum 2011 bis 2019 Fördermittel in der Höhe von rd. € 65 Mio genehmigt ${ }^{15}$. Beinahe ein Drittel dieser Summe entfällt auf die Programmlinie FEMtech Praktika für Studentinnen, jeweils etwa ein Fünftel auf Talente Praktika für Schülerinnen und Schüler, FEMtech Forschungsprojekte und Talente regional. Die restlichen rd. $8 \%$ an genehmigten Förderbarwerten verteilen sich auf Karriere-Grants, FEMtech Karriere und FEMtech Karriere-Check für KMU sowie die Programmlinie FEMtech Dissertationen, die seit dem Jahr 2014 als eigenständiges FFG-Programm „Forschungspartnerschaften - industrienahe Dissertationen“ implementiert ist.

Grafik 74 | Verteilung der Summe der genehmigten Förderbarwerte nach Programmlinie, in Prozent des Gesamtbarwerts, 2011-2019

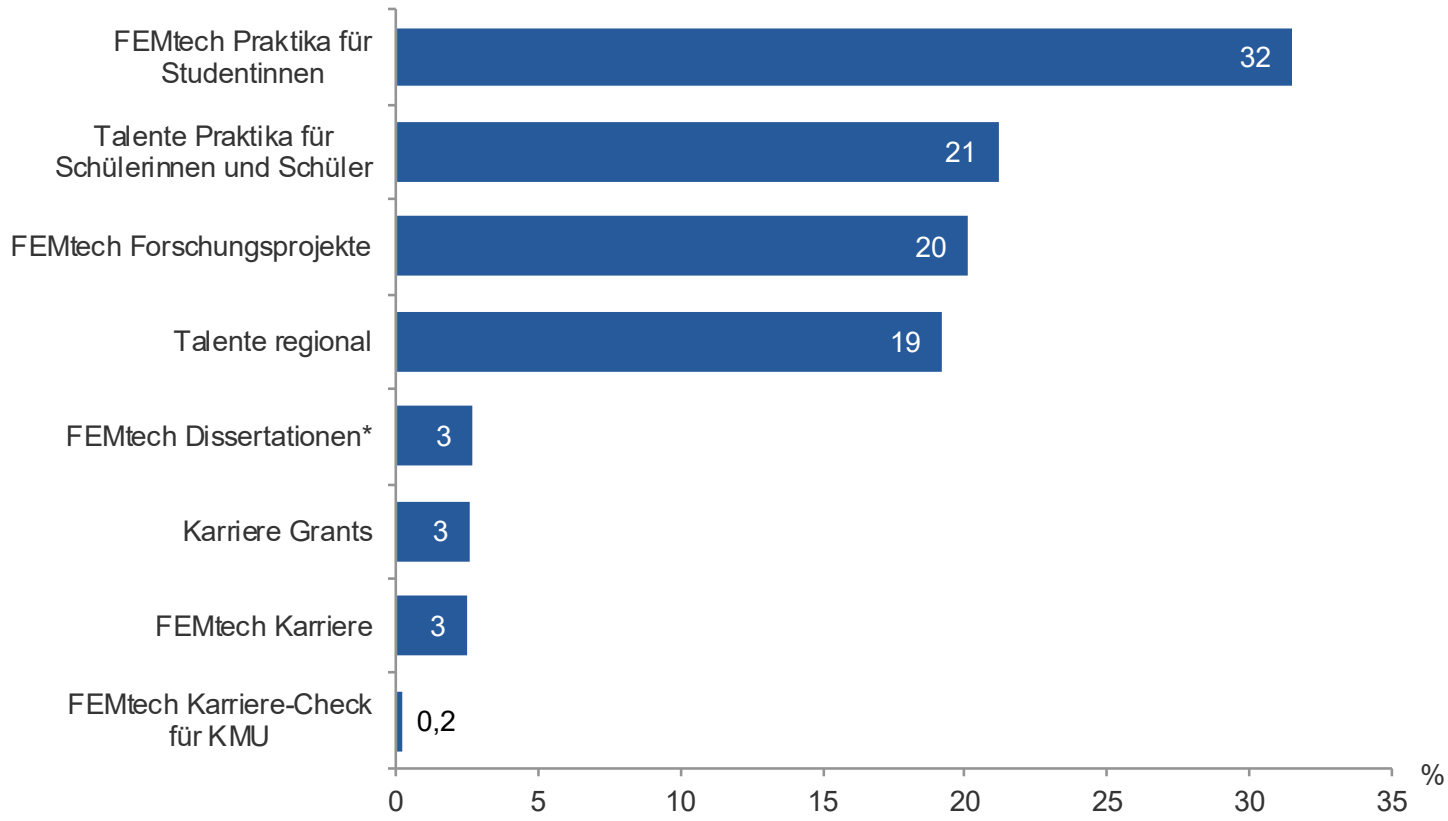

*FEMtech Dissertationen nur bis 2013

Quelle: FFG, Berechnung und Darstellung KMU Forschung Austria.

Grafik 75 zeigt die Verteilung der erstmals geförderten Organisation. Von allen im Rahmen von Talente erstmals geförderten Organisationen entfallen 58 \% auf die Programmlinie Talente Praktika für SchülerInnen. Rd. ein Fünftel der Organisationen finden über die Programmlinie FEMtech Praktika für Studentinnen und 16 \% über Talente regional ihren erstmaligen Zugang zum Programm. Sehr ähnlich verteilen sich die Organisationen, die im Rahmen ihrer Teilnahme an Talente überhaupt das erste Mal von der FFG gefördert wurden. Hier sind die Anteile der erstmals Geförderten im Vergleich zu allen in Talente erstmals Geförderten etwas niedriger bei Talente

${ }^{15}$ Dies ist nicht gleichzusetzen mit der Summe der tatsächlich ausbezahlten Fördermittel in diesem Zeitraum. 
Praktika für SchülerInnen und den FEMtech Praktika für Studentinnen und etwas höher bei Talente regional (was bedeutet, dass hier ein vergleichsweise geringerer Anteil an Organisationen bereits vor Talente einmal von der FFG gefördert wurde). Insgesamt gesehen verteilen sich die Erstförderungen somit zum überwiegenden Teil auf die drei Programmlinien Talente Praktika für SchülerInnen, FEMtech Praktika für Studentinnen und Talente regional.

Grafik 75 | Verteilung der erstmals geförderten Organisationen in Talente (ohne Karriere-Grants) sowie der Organisationen die erstmals durch ihre Teilnahme an Talente von der FFG gefördert wurden, nach Talente-Programmlinien, in Prozent

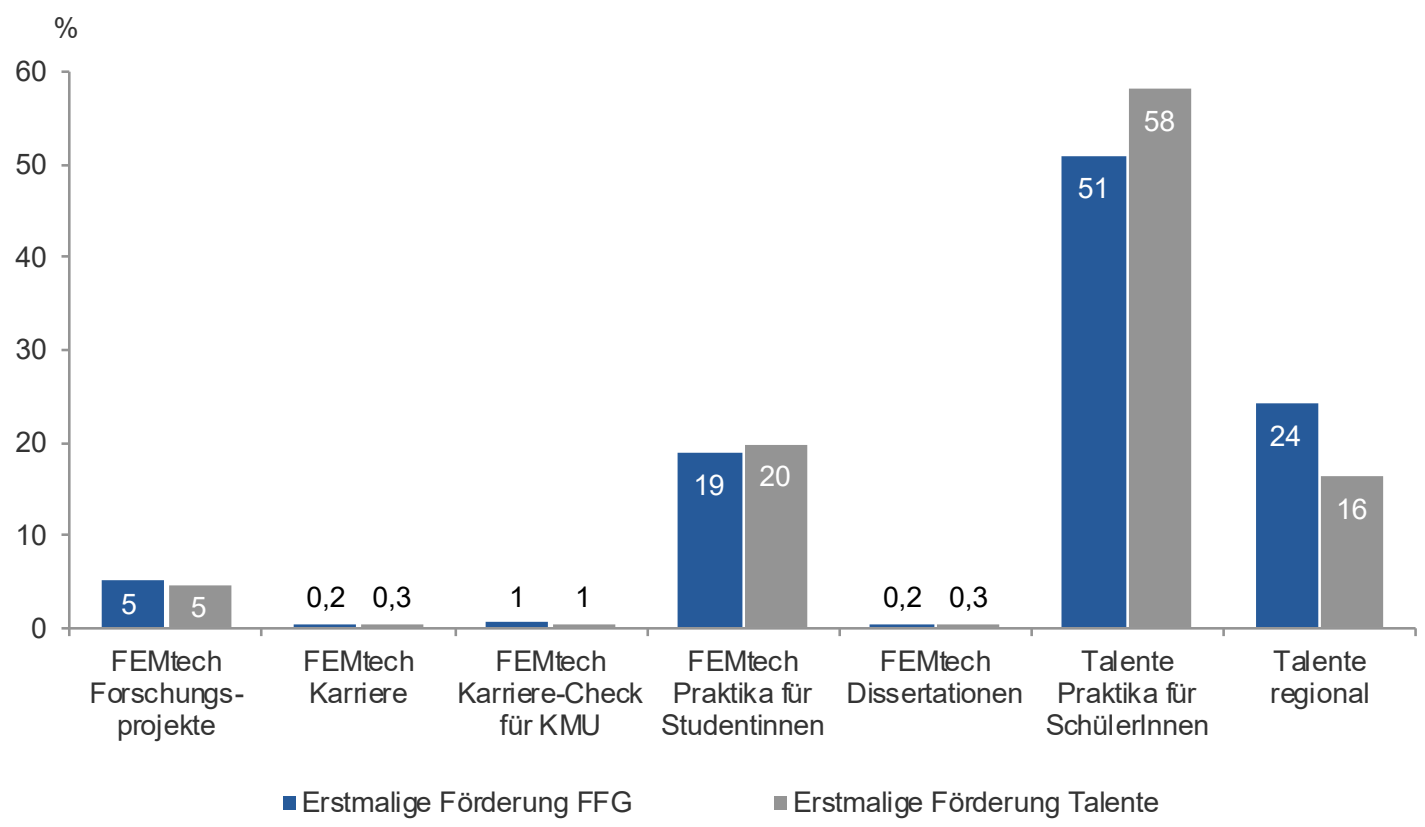

Quelle: FFG, Berechnung und Darstellung KMU Forschung Austria; erstmalige Förderung FFG $n=647$; erstmalige Förderung Talente $n=1.964$, Werte gerundet

Diese Ergebnisse sind insofern plausibel, als dass in der Programmlinie Talente regional auch Organisationen teilnehmen, die ansonsten üblicherweise nicht für Forschungsförderprogramme in Frage kommen und nicht zur typischen „FFG-Klientel“ zählen. Da viele geförderte Praktikumsplätze auf große Forschungseinrichtungen und Großunternehmen entfallen, ist es auch wahrscheinlicher, dass diese bereits an einem anderen FFG Förderprogramm teilgenommen haben.

Grafik 76 zeigt, wie hoch der Anteil der in Talente teilnehmenden Organisationen ist, die in einer, zwei, drei, vier oder fünf Programmlinien von Talente aktiv waren. Rd. $79 \%$ der Organisationen waren nur in einer, ca. $18 \%$ in zwei und etwas über $3 \%$ waren in mehr als zwei Programmlinien aktiv. 
Grafik 76 | Verteilung der geförderten Organisationen nach ihrer Teilnahme in unterschiedlichen Talente (ohne Karriere-Grants) Programmlinien*, in Prozent

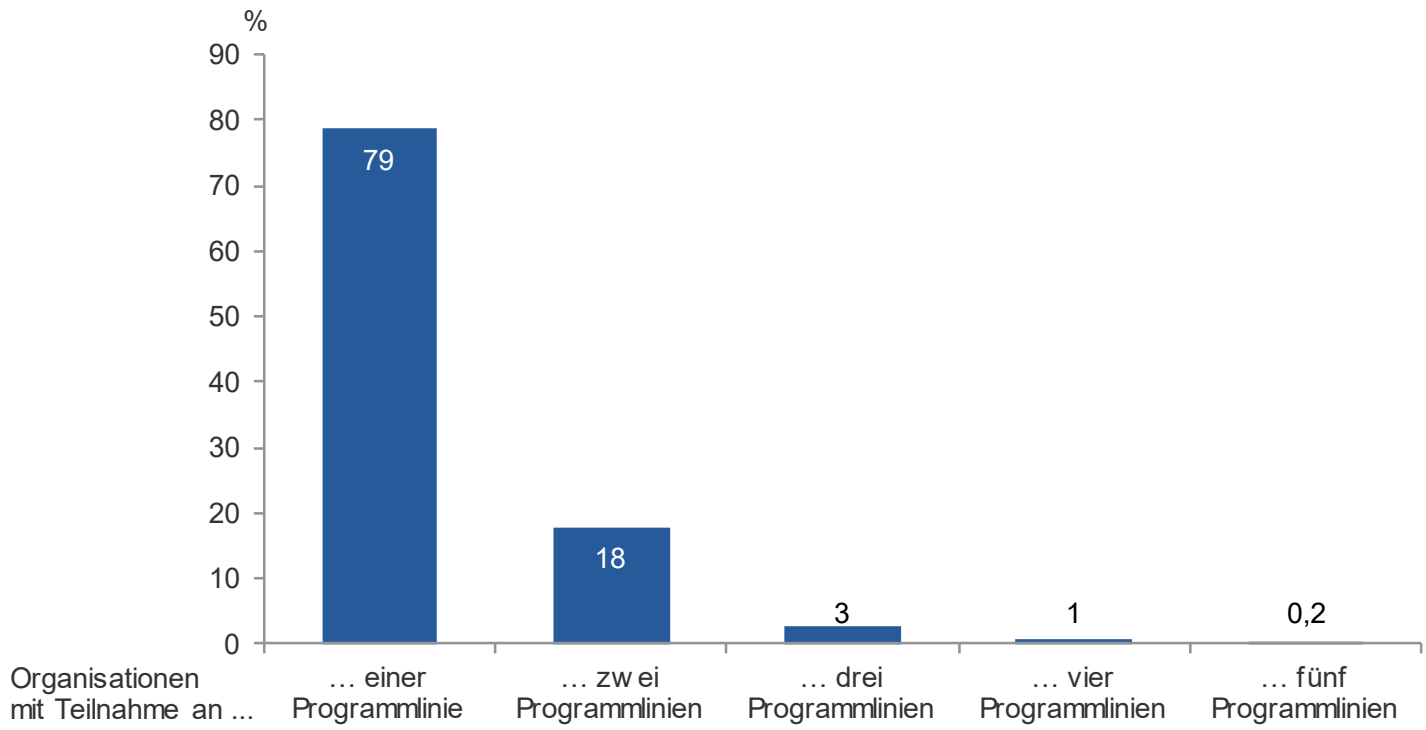

*berücksichtigt wurden Teilnahmen in sechs Programmlinien FEMtech Forschungsprojekte, FEMtech Karriere-Check für KMU, FEMtech Karriere, Talente regional, FEMtech Praktika für Studentinnen und Talente Praktika für SchülerInnen Quelle: FFG, Berechnung und Darstellung KMU Forschung Austria; n=1977, Werte gerundet

In Grafik 77 werden die Anteile der in Talente (ohne Karriere-Grants) geförderten Organisationen und ihre Teilnahmen in anderen Förderbereichen der FFG dargestellt. Das Förderportfolio der FFG kann übergeordneten Bereichen zugeordnet werden, wobei in der folgenden Darstellung die Basisprogramme, Strukturprogramme und thematischen Programme näher betrachtet werden. Im Vergleich der geförderten Organisationen in Talente (ohne Karriere-Grants) und ihrer Teilnahme an anderen Förderbereichen der FFG zeigt sich, dass rd. $36 \%$ der in Talente geförderten Organisationen nur Förderungen im Bereich der Strukturprogramme (zu dem auch das Programm Talente zählt) in Anspruch genommen hat. Immerhin 28 \% der geförderten Organisationen nahmen sowohl in Struktur-, Basisprogrammen als auch thematischen Programmen teil. Insgesamt nimmt bzw. nahm die Mehrheit der geförderten Organisationen auch an anderen Förderprogrammen im Portfolio der FFG teil. 
Grafik 77 | Überblick über andere von Talente-Teilnehmern in Anspruch genommene Förderungen aus den FFG Programmen, in Prozent

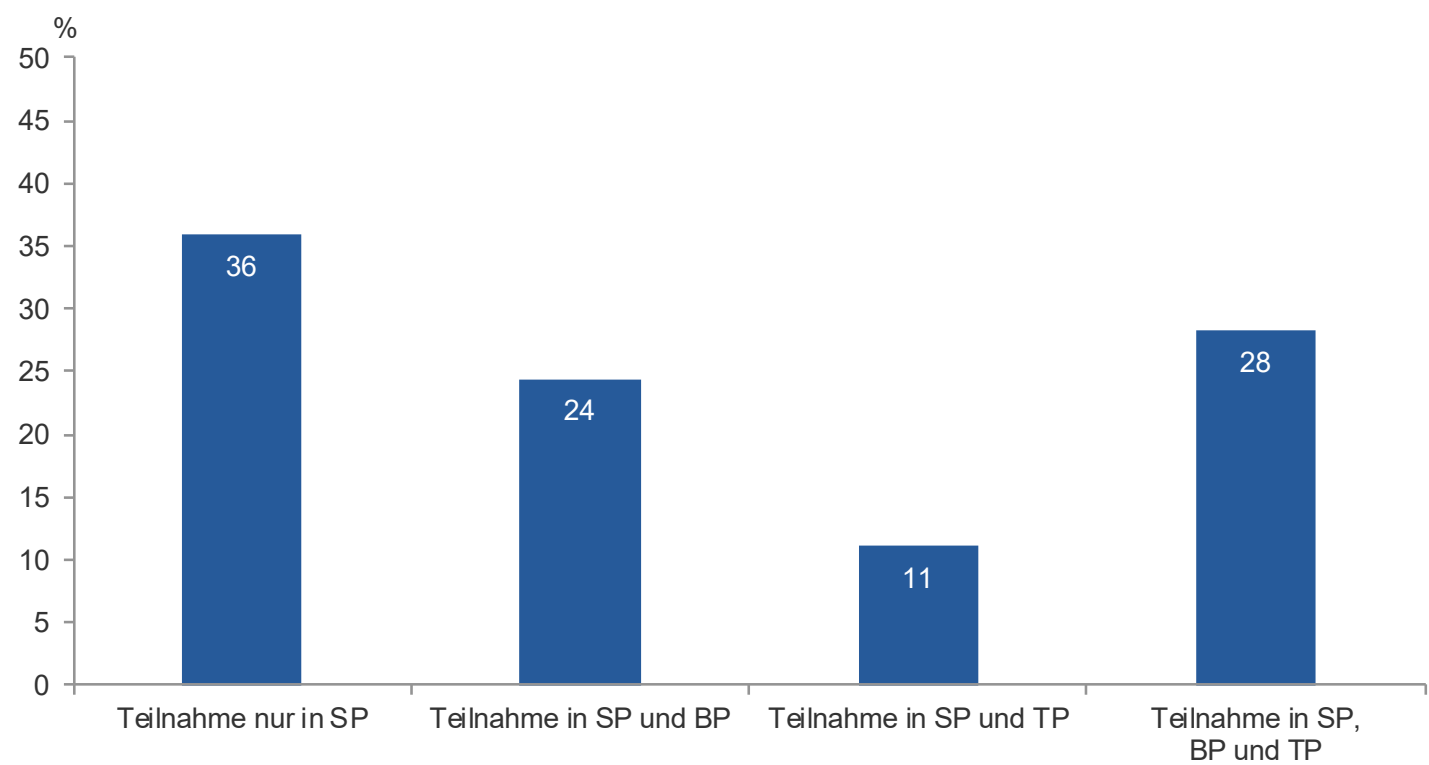

SP: Strukturprogramme, BP: Basisprogramme, TP: Thematische Programme

Quelle: FFG, Berechnung und Darstellung KMU Forschung Austria, $n=1.998$; Werte gerundet 


\section{2 | Zielerreichung}

Nachfolgend soll untersucht werden, inwiefern das Programm seine Zielsetzungen erreichen konnte. Basis dafür ist das Programmdokument ${ }^{16}$ mit der Laufzeit bis 31.12.2020 und die darin gelisteten quantitativen Indikatoren und Zielwerte für das Programm Talente, die den operativen Zielen zugeordnet wurden. Ergänzt werden diese durch weitere Indikatoren (in den Tabellen 8 bis 10 grau markiert), die geeignet scheinen, den Zielerreichungsgrad zu messen und entweder im Rahmen des Monitorings erhoben werden oder aufgrund der Evaluierung vorliegen.

Bevor der Zielerreichungsgrad der einzelnen operationalen Ziele diskutiert wird, sei auf einige Herausforderungen im diesem Zusammenhang hingewiesen, die die Zielformulierung, die Definition geeigneter Indikatoren und die Aussagekraft der Indikatoren im Programmdokument und deren Einschränkungen im Zusammenhang mit einem Humanpotentialprogramm wie Talente betreffen.

- Die formulierten Zielsetzungen adressieren persönliche Einstellungen, vorherrschende Rollenmuster und gesellschaftliche Strukturen. Sie sind daher auf der Outcome- und Impactebene verortet und nur schwer operationalisierbar. Hinzu kommen die begrenzten Einflussmöglichkeiten eines einzelnen Förderprogramms, wenn es um nichts weniger als die Änderung von vorherrschenden Strukturen und Rahmenbedingungen geht.

- Die Indikatoren im Programmdokument sind Outputindikatoren und allesamt messbar, allerdings messen sie nur sehr bedingt die Wirkungen des Programms.

- Indikatoren, die die Wirkungen des Programms besser erfassen, sind qualitativer Natur und daher nur sehr bedingt messbar zu machen (z. B. gestiegenes Interesse an Naturwissenschaft/Technik bei $x \%$ der Praktikumsteilnehmerlnnen)

Nachfolgende Tabellen 8 bis 10 geben einen Überblick über die Ziele, Instrumente, Indikatoren und den Zielerreichungsgrad je Interventionsfeld.

\section{Interventionsfeld Talente entdecken: Nachwuchs}

Dem Ziel „Interesse von Kindern und Jugendlichen (insbesondere Mädchen) an FTI steigern“ können mehrere Indikatoren im Programmdokument zugeordnet werden, die sich auf den Output der Praktika für SchülerInnen beziehen. Ersichtlich wird, dass die Zielwerte sowohl hinsichtlich der Anzahl der Praktika als auch der Anteile weiblicher Praktikantinnen und SchülerInnen aus nicht-technischen Schulen erreicht werden. Als zusätzlicher Indikator könnte das gestiegene Interesse an naturwissenschaftlich-technischen Berufen der PraktikantInnen herangezogen werden. Das Interesse wird regelmäßig im Feedback-Fragebogen erhoben und die Ergebnisse legen eine hohe Zielerreichung nahe. Ein zusätzlicher Outputindikator könnte die Anzahl der eingebundenen Kinder in Talente regional-Projekten sein, wobei hier v. a. Kinder, die direkt in die Projektaktivitäten eingebunden sind, entscheidend sind und weniger die, die indirekt über Kooperationszuschüsse erreicht werden.

${ }^{16}$ GZ 621.120/0002-III//2/2018 
Dem Ziel „FTI-Unternehmen und Forschungseinrichtungen zur Entdeckung und Förderung von zukünftigen Talenten animieren und gewinnen" können zum einen wieder der Outputindikator vergebene Praktika zugeordnet werden, wobei hier eine Differenzierung nach Praktika in Unternehmen, Universitäten und Forschungseinrichtungen und die Festlegung entsprechender Zielwerte (Anteile) empfohlen wird. Zudem kann das regelmäßig im Feedback-Fragebogen erhobene Interesse an aktiver Nachwuchsförderung als qualitativer Indikator dienen und zeigt, dass das Interesse durch das Anbieten der Praktika steigt. Im Rahmen des Instruments Talente regional kann wiederum die Anzahl der Projekte als Outputindikator dienen. Zudem könnte die Anzahl der eingebundenen Unternehmen und Forschungseinrichtungen Auskunft über die Reichweite des Instruments und damit der potentiell zu beeinflussender Organisationen geben.

Das Ziel „Vernetzung von (vor-)schulischen Bildungseinrichtungen und PartnerInnen aus Wirtschaft und Forschung initiieren" wird durch Talente regional adressiert. Hier kann wiederum die Anzahl der Projekte als Outputindikator dienen. Daneben könnte die Ausschöpfung der Kooperationszuschüsse betrachtet werden, die auch erhoben wird. Zudem kann die Nachhaltigkeit der Kooperationen untersucht werden. Das letzte Review legt hier einen hohen Zielerreichungsgrad nahe. Allerdings kann dieser Indikator nur im Rahmen von Reviews / Evaluierungen erhoben werden.

\section{Interventionsfeld Talente nützen: Chancengleichheit schaffen}

Das Ziel „Mädchen für eine naturwissenschaftlich-technische Ausbildung bzw. Frauen für eine naturwissenschaftlich-technische Berufsentscheidung motivieren" ist durch die FEMtech Praktika für Studentinnen abgedeckt. Der entsprechende Indikator ist laut Programmdokument die Anzahl der eingereichten Praktika. Der Zielwert wird mit mehr als 300 geförderten Praktika pro Jahr seit 2014 überschritten. Der Indikator sollte auf geförderte oder genehmigte Praktika geändert und der Zielwert angepasst werden. Als zusätzlicher Indikator kann das gestiegene Interesse der Praktikantinnen an einer Tätigkeit in Forschung, Naturwissenschaft und Technik dienen, der auch regelmäßig im Rahmen des Feedbacks erhoben wird. Dieser zeigt eine Interessenssteigerung bei mehr als zwei Drittel der Teilnehmerinnen.

„Faire Rahmenbedingungen für Frauen und Männer in FTI-Unternehmen schaffen“ ist als Ziel dem Instrument FEMtech Karriere zuzuordnen. Indikatoren laut Programmdokument sind die eingereichten bzw. geförderten Projekte. Der entsprechende Zielwert wurde bis auf ein (Einreichung) bzw. zwei Jahre (Förderung) verfehlt. Bezüglich FEMtech Karriere-Check konnte der Zielwert zu den geförderten Projekten in einem Jahr erreicht werden, im anderen Jahr wurde er verfehlt. Zur Beurteilung des Instruments wurden zwei weitere qualitative Indikatoren herangezogen. Die gestiegene Akzeptanz und Awareness für das Thema Gleichstellung sowie die Installierung einer/s Gleichstellungsbeauftragten in den geförderten Organisationen. Hier kann von einer Zielerreichung ausgegangen werden. Der erste Indikator könnte nur im Rahmen eines Reviews / einer Evaluierung erhoben werden, letzter in Rahmen der Berichtslegung der Fördernehmerlnnen. 
Das Ziel „Erhöhung des Anteils von Frauen auf allen Hierarchieebenen sowie Erhöhung ihrer Karrierechancen in den geförderten Unternehmen" ist in Hinblick auf die Erhöhung des Frauenanteils gut operationalisierbar. Als Indikatoren böten sich z. B. an: Weiterbeschäftigungsanteile der Studentinnen nach Praktikumsende, der regelmäßig im Feedback-Fragebogen erhoben wird. Die Festsetzung realistischer Zielwerte sind Voraussetzung für die Validität. Im Rahmen von FEMtech Karriere könnte der Frauenanteil der geförderten Organisationen nach Hierarchiestufen zu Projektbeginn mit dem Frauenanteil nach Projektende bzw. nach mehreren Jahren gegenübergestellt werden. Dies muss allerdings im Rahmen eines Reviews bzw. einer Evaluierung erhoben werden. Derzeit kann das Ziel nur sehr bedingt erreicht werden.

„Die Initiierung von FTI-Vorhaben mit genderrelevanten Inhalten“ wird über die Förderung von FEMtech Forschungsprojekten realisiert. Auch hier kommt ein Outputindikator zum Einsatz. Der Zielwert der eingereichten Projekte konnte ab 2012 erreicht werden, bei den geförderten Projekten nicht. Da die Initiierung wohl in erster Linie über durchgeführte Projekte erfolgt, ist der Indikator Anzahl geförderte Projekte deutlich aussagekräftiger.

\section{Interventionsfeld Talente finden: Forscherinnen und Forscher}

Das Ziel „ForscherInnen aus dem In- und Ausland für die anwendungsorientierte Forschung in Österreich gewinnen" kann in Hinblick auf ForscherInnen aus dem Ausland dem Instrument Karriere-Grants zugeordnet werden. Hierfür wird der Indikator Anzahl der eingereichten und vergebenen Karriere-Grants im Programmdokument verwendet. Die Zielerreichung ist in Hinblick auf den Indikator gegeben, allerdings stellt sich die Frage nach der Validität. Eine Differenzierung nach Granttyp ist empfehlenswert, wobei in erster Linie der Relocation Grant von Bedeutung ist. Wichtiger noch scheint aber der Beitrag der Förderung, $d \mathrm{~h}$. inwiefern die Förderung dazu beiträgt, dass ForscherInnen nach Österreich kommen. Im Zuge der Evaluierung wurde erhoben, wie hoch der Anteil an den FördernehmerInnen des Relocation Grant ist, die ohne Förderung nicht nach Österreich gekommen wären - dieser ist minimal. Der Indikator könnte bei Antragsstellung abgefragt werden.

Die Jobbörse kann zum Ziel „Forscherlnnen an die F\&E-Unternehmen heranzuführen und zu binden" beitragen. Der dazugehörige Indikator der Anzahl der Nutzerlnnen ist nicht sehr aussagekräftig. Der Zielwert wurde nicht erreicht. Als weiterer Indikator für dieses Ziel bietet sich die Anzahl der vergebenen Interview-Grants an, die Auskunft darüber geben, ob ForscherInnen aus dem Ausland an österreichischen Unternehmen und Forschungseinrichtungen herangeführt wurden. 
Tabelle 8 | Zielerreichung Interventionsfeld Talente entdecken - Nachwuchs

\begin{tabular}{|c|c|c|c|c|c|c|}
\hline Ziel & Instrument & $\begin{array}{l}\text { Indikator und Wirkungs- } \\
\text { ebene }\end{array}$ & Zielwert & Ergebnis & Zielerreichung & Anmerkungen \\
\hline \multirow{5}{*}{$\begin{array}{l}\text { Interesse von Kindern } \\
\text { und Jugendlichen (insbe- } \\
\text { sondere von Mädchen } \\
\text { und jungen Frauen) an } \\
\text { FTI steigern und ihren na- } \\
\text { turwissenschaftlich-tech- } \\
\text { nischen Bezug durch die } \\
\text { aktive Einbindung in Pro- } \\
\text { jekte vertiefen. }\end{array}$} & \multirow{4}{*}{$\begin{array}{l}\text { Praktika für Schü- } \\
\text { lerInnen }\end{array}$} & $\begin{array}{l}\text { Anzahl eingereichte bzw. } \\
\text { vergebene Praktika } \\
\text { (Output) }\end{array}$ & $\begin{array}{l}1.000 \text { bis } 1.500 \text { Praktika } \\
\text { pro Ausschreibung nach } \\
\text { Maßgabe der verfügbaren } \\
\text { Mittel }\end{array}$ & $\begin{array}{l}\text { Je nach Ausschreibung } \\
\text { mind. } 1.270 \text { bis max. } \\
1.710\end{array}$ & $\mathrm{Ja}$ & \\
\hline & & $\begin{array}{l}\text { Anzahl der Mädchen } \\
\text { (Output) }\end{array}$ & $\begin{array}{l}\text { Erhöhung des Mädchen- } \\
\text { anteils (2011-2014 durch- } \\
\text { schnittlich } 35 \%)\end{array}$ & $\begin{array}{l}\text { Mädchenanteil steigt } \\
\text { kontinuierlich von 32\% } \\
2011 \text { auf } 49 \% 2019\end{array}$ & $\mathrm{Ja}$ & $\begin{array}{l}\text { Quotenregelung wirk- } \\
\text { sam }\end{array}$ \\
\hline & & $\begin{array}{l}\text { Art der Schulen } \\
\text { (Output) }\end{array}$ & $\begin{array}{l}\text { Erhöhung des Anteils von } \\
\text { nicht-technischen Schulen }\end{array}$ & $\begin{array}{l}\text { Anteil der AHS steigt } \\
\text { kontinuierlich von 28\%- } \\
29 \%(2011-2014) \text { auf } \\
53 \% 2019\end{array}$ & $\mathrm{Ja}$ & $\begin{array}{l}\text { Quotenregelung wirk- } \\
\text { sam }\end{array}$ \\
\hline & & $\begin{array}{l}\text { Interesse der SchülerInnen } \\
\text { für Naturwissenschaft/Tech- } \\
\text { nik } \\
\text { (Outcome) }\end{array}$ & $\begin{array}{l}\text { Substanzielle Steigerung } \\
\text { des Interesse der Schüle- } \\
\text { rInnen (qualitativer Indika- } \\
\text { tor, Zielwert definieren) }\end{array}$ & $\begin{array}{l}\text { Gestiegenes Interesse } \\
\text { an naturwissenschaft- } \\
\text { lich-technischen Beru- } \\
\text { fen bei } 57 \%-73 \% 2011 \\
-2019 \\
\text { (Feedback Schülerln- } \\
\text { nen) }\end{array}$ & $\begin{array}{l}\text { In Abhängigkeit } \\
\text { von Zielwert } \\
\text { Qualitative Ein- } \\
\text { schätzung: ja, }\end{array}$ & $\begin{array}{l}\text { Indikator wird regel- } \\
\text { mäßig über Feed- } \\
\text { back-Fragebögen er- } \\
\text { hoben, daher Monito- } \\
\text { ring möglich }\end{array}$ \\
\hline & Talente regional & $\begin{array}{l}\text { Anzahl der erreichten Kin- } \\
\text { der und Jugendlichen pro } \\
\text { Projekt } \\
\text { (Output) }\end{array}$ & $\begin{array}{l}\text { Einbindung möglichst vie- } \\
\text { ler Kinder und Jugendliche } \\
\text { in die Talente regional Pro- } \\
\text { jekte (Zielwert zu definie- } \\
\text { ren) }\end{array}$ & $\begin{array}{l}\text { Zwischen rd. } 280 \text { und rd. } \\
450 \text { Kinder und Jugend- } \\
\text { liche pro Projekt } 2011 \text { - } \\
2016 \text { erreicht }\end{array}$ & $\begin{array}{l}\text { In Abhängigkeit } \\
\text { von Zielwert }\end{array}$ & $\begin{array}{l}\text { Einbindung in Projek- } \\
\text { taktivitäten entschei- } \\
\text { dend (nicht Einbin- } \\
\text { dung über Kooperati- } \\
\text { onszuschüsse) }\end{array}$ \\
\hline
\end{tabular}




\begin{tabular}{|c|c|c|c|c|c|c|}
\hline Ziel & Instrument & $\begin{array}{l}\text { Indikator und Wirkungs- } \\
\text { ebene }\end{array}$ & Zielwert & Ergebnis & Zielerreichung & Anmerkungen \\
\hline \multirow{4}{*}{$\begin{array}{l}\text { FTI-Unternehmen und } \\
\text { Forschungseinrichtun- } \\
\text { gen zur Entdeckung } \\
\text { und Förderung von zu- } \\
\text { künftigen Talenten ani- } \\
\text { mieren und gewinnen. }\end{array}$} & \multirow{2}{*}{$\begin{array}{l}\text { Praktika für Schü- } \\
\text { lerlnnen }\end{array}$} & $\begin{array}{l}\text { Anzahl eingereichte bzw. } \\
\text { vergebene Praktika } \\
\text { (Output) }\end{array}$ & $\begin{array}{l}1.000 \text { bis } 1.500 \text { Praktika pro } \\
\text { Ausschreibung nach Maß- } \\
\text { gabe der verfügbaren Mittel }\end{array}$ & $\begin{array}{l}\text { Je nach Ausschreibung } \\
\text { mind. } 1.270 \text { bis max. } 1.710\end{array}$ & Ja & $\begin{array}{l}\text { Differenzierung nach } \\
\text { Praktika in Unterneh- } \\
\text { men / Universitäten / } \\
\text { Forschungseinrich- } \\
\text { tungen empfohlen }\end{array}$ \\
\hline & & $\begin{array}{l}\text { Interesse der Unterneh- } \\
\text { men an aktiver Nach- } \\
\text { wuchsförderung (Out- } \\
\text { come) }\end{array}$ & $\begin{array}{l}\text { Interesse der Unternehmen } \\
\text { an aktiver Nachwuchsförde- } \\
\text { rung steigern (qualitativer In- } \\
\text { dikator, Zielwert definieren) }\end{array}$ & $\begin{array}{l}\text { Gestiegenes Interesse an } \\
\text { der aktiven Nachwuchsför- } \\
\text { derung bei } 73 \%-81 \% 2011 \\
\text { - } 2019 \text { (Feedback Förder- } \\
\text { nehmerInnen) }\end{array}$ & $\begin{array}{l}\text { In Abhängigkeit } \\
\text { von Zielwert } \\
\text { Qualitative Ein- } \\
\text { schätzung: ja, }\end{array}$ & $\begin{array}{l}\text { Indikator wird regel- } \\
\text { mäßig über Feed- } \\
\text { back-Fragebögen er- } \\
\text { hoben, daher Monito- } \\
\text { ring möglich }\end{array}$ \\
\hline & \multirow{2}{*}{ Talente regional } & $\begin{array}{l}\text { Anzahl eingereichter bzw. } \\
\text { geförderter Projekte von } \\
\text { Talente regional } \\
\text { (Output) }\end{array}$ & $\begin{array}{l}\text { 5-20 eingereichte bzw. 11-15 } \\
\text { geförderte Projekte pro Aus- } \\
\text { schreibung }\end{array}$ & $\begin{array}{l}34-81 \text { eingereichte Pro- } \\
\text { jekte } 2011-2019 \\
11-24 \text { geförderte Projekte } \\
2011-2019\end{array}$ & ja & \\
\hline & & $\begin{array}{l}\text { Eingebundene Unterneh- } \\
\text { men und Forschungsein- } \\
\text { richtungen } \\
\text { (Output) }\end{array}$ & $\begin{array}{l}\text { Anzahl eingebundener Unter- } \\
\text { nehmen und Forschungsein- } \\
\text { richtungen erhöhen } \\
\text { (Zielwert zu definieren) }\end{array}$ & $\begin{array}{l}\text { 23-53 Unternehmen, } \\
\text { 6-20 Forschungseinrichtun- } \\
\text { gen und 13-34 Hochschu- } \\
\text { len }\end{array}$ & $\begin{array}{l}\text { In Abhängigkeit } \\
\text { von Zielwert }\end{array}$ & Monitoring möglich \\
\hline \multirow{3}{*}{$\begin{array}{l}\text { Vernetzung von (vor- } \\
\text { )schulischen Bildungs- } \\
\text { einrichtungen und Part- } \\
\text { nerlnnen aus Wirt- } \\
\text { schaft und Forschung } \\
\text { initiieren }\end{array}$} & \multirow{3}{*}{ Talente regional } & $\begin{array}{l}\text { Anzahl eingereichter bzw. } \\
\text { geförderter Projekte von } \\
\text { Talente regional } \\
\text { (Output) }\end{array}$ & $\begin{array}{l}\text { 5-20 eingereichte bzw. 11-15 } \\
\text { geförderte Projekte pro Aus- } \\
\text { schreibung }\end{array}$ & $\begin{array}{l}34-81 \text { eingereichte Pro- } \\
\text { jekte } 2011-2019 \\
11-24 \text { geförderte Projekte } \\
2011-2019\end{array}$ & ja & $\begin{array}{l}\text { Bedingt geeignet als } \\
\text { Indikator für Vernet- } \\
\text { zung }\end{array}$ \\
\hline & & $\begin{array}{l}\text { Ausschöpfung der Ko- } \\
\text { operationszuschüsse zur } \\
\text { Einbindung } 10 \text { weiterer } \\
\text { Bildungseinrichtungen } \\
\text { (Output) }\end{array}$ & $\begin{array}{l}\text { Ausschöpfung der Kooperati- } \\
\text { onszuschüsse } \\
\text { (Zielwert zu definieren) }\end{array}$ & $\begin{array}{l}78 \%-100 \% \text { der Kooperati- } \\
\text { onszuschüsse } 2011-2016 \\
\text { ausgeschöpft }\end{array}$ & $\begin{array}{l}\text { In Abhängigkeit } \\
\text { von Ausschöp- } \\
\text { fung hoch }\end{array}$ & \\
\hline & & $\begin{array}{l}\text { Nachhaltigkeit der Koope- } \\
\text { rationen } \\
\text { (Outcome) }\end{array}$ & $\begin{array}{l}\text { Kooperationen mit einzelnen } \\
\text { Organisationen auch nach } \\
\text { Projektende erhalten (quali- } \\
\text { tativer Indikator oder Zielwert } \\
\text { definieren) }\end{array}$ & $\begin{array}{l}72 \% \text { nachhaltige Kooperati- } \\
\text { onen mit einzelnen Organi- } \\
\text { sationen (ibw-Befragung } \\
\text { der Projektteilnehmerlnnen } \\
\text { bei Review 2018) }\end{array}$ & $\begin{array}{l}\text { In Abhängigkeit } \\
\text { von Zielwert } \\
\text { Qualitative Ein- } \\
\text { schätzung: ja }\end{array}$ & $\begin{array}{l}\text { nur im Rahmen einer } \\
\text { Befragung zu erhe- } \\
\text { ben, kein regelmäßi- } \\
\text { ges Monitoring mög- } \\
\text { lich }\end{array}$ \\
\hline
\end{tabular}


Tabelle 9 | Zielerreichung Interventionsfeld Talente nützen - Chancengleichheit

\begin{tabular}{|c|c|c|c|c|c|c|}
\hline Ziele & Instrument & $\begin{array}{l}\text { Indikator und Wirkungs- } \\
\text { ebene }\end{array}$ & Zielwert & Ergebnis & Zielerreichung & Anmerkungen \\
\hline \multirow{2}{*}{$\begin{array}{l}\text { Mädchen für eine natur- } \\
\text { wissenschaftlich-techni- } \\
\text { sche Ausbildung bzw. } \\
\text { Frauen für eine naturwis- } \\
\text { senschaftlich-technische } \\
\text { Berufsentscheidung mo- } \\
\text { tivieren }\end{array}$} & \multirow{2}{*}{$\begin{array}{l}\text { FEMtech Praktika } \\
\text { für Studentinnen }\end{array}$} & $\begin{array}{l}\text { Anzahl der eingereichten } \\
\text { FEMtech Praktika für Stu- } \\
\text { dentinnen } \\
\text { (Output) }\end{array}$ & $\begin{array}{l}200-300 \text { pro Ausschrei- } \\
\text { bung nach Maßgabe der } \\
\text { verfügbaren Mittel }\end{array}$ & $\begin{array}{l}\text { Ab } 2014 \text { wurden pro Aus- } \\
\text { schreibung mehr als } 300 \\
\text { Förderungen vergeben }\end{array}$ & ja & $\begin{array}{l}\text { Anpassung: geneh- } \\
\text { migte oder geförderte } \\
\text { Prakika } \\
\text { Eventuell Zielwert er- } \\
\text { höhen }\end{array}$ \\
\hline & & $\begin{array}{l}\text { Interesse an Tätigkeit in } \\
\text { Forschung, Naturwissen- } \\
\text { schaft und Technik } \\
\text { (Outcome) }\end{array}$ & $\begin{array}{l}\text { Interesse der Studentin- } \\
\text { nen steigern } \\
\text { (qualitativer Indikator, } \\
\text { Zielwert zu definieren) }\end{array}$ & $\begin{array}{l}\text { Gestiegenes Interesse an } \\
\text { Naturwissenschaften bei } \\
69 \%-75 \% 2011-2019\end{array}$ & $\begin{array}{l}\text { In Abhängigkeit } \\
\text { von Zielwert } \\
\text { Qualitative Ein- } \\
\text { schätzung: ja, }\end{array}$ & $\begin{array}{l}\text { Indikator wird regel- } \\
\text { mäßig über Feed- } \\
\text { back-Fragebögen er- } \\
\text { hoben, daher Monito- } \\
\text { ring möglich }\end{array}$ \\
\hline \multirow{4}{*}{$\begin{array}{l}\text { Faire Rahmenbedingun- } \\
\text { gen für Frauen und Män- } \\
\text { ner in FTI-Unternehmen } \\
\text { schaffen }\end{array}$} & FEMtech Karriere & $\begin{array}{l}\text { Anzahl der eingereichten } \\
\text { bzw. geförderten FEMtech } \\
\text { Karriere Projekte } \\
\text { (Output) }\end{array}$ & $\begin{array}{l}\text { 10-15 eingereichte bzw. } \\
5-10 \text { geförderte Projekte } \\
\text { pro Jahr }\end{array}$ & $\begin{array}{l}\text { 6-12 Einreichungen (Ge- } \\
\text { wünschte Anzahl nur } \\
2016) \\
1-10 \quad \text { Projekte (Ge- } \\
\text { wünschte Anzahl nur } \\
2016 \text { und 2018) }\end{array}$ & $\begin{array}{l}\text { Teilweise } \\
\text { Einreichungen nur } \\
2016, \\
\text { Geförderte nur } \\
2016 \text { / } 2018\end{array}$ & \\
\hline & $\begin{array}{l}\text { FEMtech Karriere- } \\
\text { Check für KMU }\end{array}$ & $\begin{array}{l}\text { Anzahl der geförderten } \\
\text { FEMtech Karriere-Check } \\
\text { für KMU Projekte } \\
\text { (Output) }\end{array}$ & $\begin{array}{l}\text { 10-20 geförderte Projekte } \\
\text { pro Kalenderjahr }\end{array}$ & $\begin{array}{l}\text { 2015: } 6 \text { Projekte } \\
\text { 2016: } 11 \text { Projekte }\end{array}$ & teilweise & \\
\hline & $\begin{array}{l}\text { FEMtech Karriere } \\
\text { (Outcome) }\end{array}$ & $\begin{array}{l}\text { Akzeptanz und Awareness } \\
\text { für das Thema Gleichstel- } \\
\text { lung }\end{array}$ & $\begin{array}{l}\text { Gestiegene Akzeptanz } \\
\text { und Awareness für das } \\
\text { Thema Gleichstellung } \\
\text { (qualitativer Indikator) }\end{array}$ & $\begin{array}{l}\text { Erhöhte Akzeptanz bei } 16 \\
\text { von } 17 \text { Befragten laut Be- } \\
\text { fragung der KMU For- } \\
\text { schung Austria }\end{array}$ & $\begin{array}{l}\text { Qualitative Ein- } \\
\text { schätzung: Ja }\end{array}$ & $\begin{array}{l}\text { nur im Rahmen einer } \\
\text { Befragung oder in In- } \\
\text { terviews zu erheben, } \\
\text { kein regelmäßiges } \\
\text { Monitoring möglich }\end{array}$ \\
\hline & $\begin{array}{l}\text { FEMtech Karriere } \\
\text { (Outcome) }\end{array}$ & $\begin{array}{l}\text { Nachhaltige Berücksichti- } \\
\text { gung von Gender } \\
\text { Mainstreaming }\end{array}$ & $\begin{array}{l}\text { Installierung einer } \\
\text { Gendervertrauensperson } \\
\text { oder einer/s Gleichstel- } \\
\text { lungsbeauftragten } \\
\text { Zielwert zu definieren) }\end{array}$ & $\begin{array}{l}11 \text { von } 17 \text { Befragten in- } \\
\text { stallierten eine Gender- } \\
\text { vertrauensperson oder } \\
\text { ein/e Gleichstellungsbe- } \\
\text { aufte/n laut Befragung der } \\
\text { KMU Forschung Austria }\end{array}$ & $\begin{array}{l}\text { Qualitative Ein- } \\
\text { schätzung: Ja }\end{array}$ & $\begin{array}{l}\text { kann Rahmen der } \\
\text { Projektendberichte } \\
\text { erhoben werden }\end{array}$ \\
\hline
\end{tabular}




\begin{tabular}{|c|c|c|c|c|c|c|}
\hline Ziele & Instrument & $\begin{array}{l}\text { Indikator und Wirkungs- } \\
\text { ebene }\end{array}$ & Zielwert & Ergebnis & Zielerreichung & Anmerkungen \\
\hline \multirow{2}{*}{$\begin{array}{l}\text { Erhöhung des Anteils } \\
\text { von Frauen auf allen Hie- } \\
\text { rarchieebenen sowie Er- } \\
\text { höhung ihrer Karrierech- } \\
\text { ancen in den geförderten } \\
\text { Unternehmen }\end{array}$} & $\begin{array}{l}\text { FEMtech Praktika } \\
\text { für Studentinnen }\end{array}$ & $\begin{array}{l}\text { Weiterbeschäftigung der } \\
\text { Studentinnen nach Prakti- } \\
\text { kumsende } \\
\text { (Outcome) }\end{array}$ & $\begin{array}{l}\text { Anteil der Weiterbeschäf- } \\
\text { tigung der Studentinnen } \\
\text { in den geförderten Unter- } \\
\text { nehmen (Zielwert zu defi- } \\
\text { nieren) }\end{array}$ & $\begin{array}{l}\text { Weiterbeschäftigung laut } \\
\text { Fördernehmerlnnen zwi- } \\
\text { schen } 7 \% \text { und } 23 \% 2011 \\
-2019 \\
\text { Dienstverhältnis fortset- } \\
\text { zen laut Studentinnen } \\
\text { zwischen 14\% und } 28 \% \\
2011-2019\end{array}$ & $\begin{array}{l}\text { In Abhängigkeit } \\
\text { von Zielwert }\end{array}$ & $\begin{array}{l}\text { Indikator wird regel- } \\
\text { mäßig über Feed- } \\
\text { back-Fragebögen er- } \\
\text { hoben, daher Monito- } \\
\text { ring möglich }\end{array}$ \\
\hline & FEMtech Karriere & $\begin{array}{l}\text { Erhöhung des Frauenan- } \\
\text { teils insgesamt und in Füh- } \\
\text { rungspositionen } \\
\text { (Outcome) }\end{array}$ & $\begin{array}{l}\text { Steigerung des Frauen- } \\
\text { anteils in den geförderten } \\
\text { Unternehmen insgesamt } \\
\text { und in Führungspositio- } \\
\text { nen (Zielwerte zu definie- } \\
\text { ren) }\end{array}$ & $\begin{array}{l}4 \text { von } 10 \text { Unternehmen } \\
\text { mit tatsächlichen Steige- } \\
\text { rungen der Frauenanteile } \\
\text { laut Befragung der Pro- } \\
\text { jektteilnehmerInnen }\end{array}$ & $\begin{array}{l}\text { In Abhängigkeit } \\
\text { von Zielwert, } \\
\text { qualitative Ein- } \\
\text { schätzung eher } \\
\text { nein }\end{array}$ & $\begin{array}{l}\text { nur im Rahmen einer } \\
\text { Befragung oder in In- } \\
\text { terviews zu erheben, } \\
\text { kein regelmäßiges } \\
\text { Monitoring möglich }\end{array}$ \\
\hline $\begin{array}{l}\text { Initiierung von FTI-Vor- } \\
\text { haben mit genderrele- } \\
\text { vanten Inhalten }\end{array}$ & $\begin{array}{l}\text { FEMtech For- } \\
\text { schungsprojekte }\end{array}$ & $\begin{array}{l}\text { Anzahl der eingereichten } \\
\text { bzw. geförderten FEMtech } \\
\text { Forschungsprojekte } \\
\text { (Output) }\end{array}$ & $\begin{array}{l}20-30 \text { eingereichte bzw. } \\
10-12 \text { geförderte Projekte } \\
\text { pro Ausschreibung }\end{array}$ & $\begin{array}{l}\text { Einreichungen ab } 2012 \\
\text { überplanmäßig erfüllt (35- } \\
54 \text { Einreichungen) } \\
\text { Geförderte Projekte pro } \\
\text { Ausschreibung: } 8 \text { bis } 9 \\
\text { Projekte }\end{array}$ & $\begin{array}{l}\text { teilweise } \\
\text { Ja in Bezug auf } \\
\text { Einreichungen, } \\
\text { nein in Bezug auf } \\
\text { geförderte Pro- } \\
\text { jekte }\end{array}$ & $\begin{array}{l}\text { Eine Initiierung der } \\
\text { nicht geförderten } \\
\text { FEMtech For- } \\
\text { schungsprojekte ist } \\
\text { fraglich. } \\
\text { Zentral sind die geför- } \\
\text { derten Projekte. }\end{array}$ \\
\hline
\end{tabular}


Tabelle 10 | Zielerreichung Interventionsfeld Talente nützen - Forscherinnen und Forscher

\begin{tabular}{|c|c|c|c|c|c|c|}
\hline Ziele & Instrument & $\begin{array}{l}\text { Indikator und Wirkungs- } \\
\text { ebene }\end{array}$ & Zielwert & Ergebnis & Zielerreichung & Anmerkung \\
\hline \multirow{2}{*}{$\begin{array}{l}\text { ForscherInnen aus } \\
\text { dem In- und Ausland } \\
\text { für die anwendungsori- } \\
\text { entierte Forschung in } \\
\text { Österreich gewinnen }\end{array}$} & \multirow[t]{2}{*}{ Karriere Grants } & $\begin{array}{l}\text { Anzahl der eingereichten } \\
\text { bzw. vergebenen Karriere- } \\
\text { Grants } \\
\text { (Output) }\end{array}$ & $\begin{array}{l}\text { Erhöhung der vergebe- } \\
\text { nen Karriere-Grants pro } \\
\text { Kalenderjahr (2012-2014 } \\
\text { durchschnittlich } 180 \text { Kar- } \\
\text { riere-Grants/Kalender- } \\
\text { jahr) }\end{array}$ & $\begin{array}{l}\text { von } 2012 \text { bis } 2014 \text { wur- } \\
\text { den durchschnittlich } 171 \\
\text { Karriere-Grants verge- } \\
\text { ben; für den Zeitraum } \\
2011 \text { bis } 2019 \text { waren es } \\
\text { durchschnittlich } 211\end{array}$ & ja & $\begin{array}{l}\text { Zeigt, dass Forsche- } \\
\text { rlnnen aus dem } \\
\text { Ausland gewonnen } \\
\text { wurden (bei Reloca- } \\
\text { tion Grant). } \\
\text { Zumindest Differen- } \\
\text { zierung nach } \\
\text { Granttyp empfohlen. }\end{array}$ \\
\hline & & $\begin{array}{l}\text { Anteil der Relocation } \\
\text { Grants Fördernehmerln- } \\
\text { nen, die ohne Förderung } \\
\text { nicht gekommen wären } \\
\text { (Outcome) }\end{array}$ & Zielwert zu definieren & $\begin{array}{l}\text { 5\% der befragten Reloca- } \\
\text { tion Grant Fördernehme- } \\
\text { rInnen wären wahrschein- } \\
\text { lich nicht oder nicht nach } \\
\text { Österreich gekommen; }\end{array}$ & $\begin{array}{l}\text { Qualitative Ein- } \\
\text { schätzung: nein }\end{array}$ & $\begin{array}{l}\text { Zeigt, inwiefern die } \\
\text { Förderung aus- } \\
\text { schlaggebend war, } \\
\text { um nach Österreich } \\
\text { zu kommen } \\
\text { Monitoring bei An- } \\
\text { tragstellung möglich }\end{array}$ \\
\hline \multirow{2}{*}{$\begin{array}{l}\text { Forscherlnnen an die } \\
\text { F\&E-Unternehmen } \\
\text { heranführen und bin- } \\
\text { den }\end{array}$} & Jobbörse & $\begin{array}{l}\text { Anzahl Nutzerlnnen der } \\
\text { Jobbörse (Output) }\end{array}$ & $\begin{array}{l}\text { Konstanthaltung von } \\
\text { durchschnittlich } 6000 \text { Uni- } \\
\text { que Visitors pro Quartal }\end{array}$ & $\begin{array}{l}\text { Im Zeitraum } 2013 \text { bis } \\
2018 \text { beträgt die Anzahl } \\
\text { der Unique Visitors pro } \\
\text { Quartal durchschnittlich } \\
5.220 \text { (ohne } 3 . \text { Quartal } \\
2016 \text { ) }\end{array}$ & nein & $\begin{array}{l}\text { Die Nutzung der } \\
\text { Jobbörse ist wenig } \\
\text { geeignet zu zeigen, } \\
\text { inwiefern Forsche- } \\
\text { rlnnen tatsächlich } \\
\text { an Unternehmen } \\
\text { herangeführt wer- } \\
\text { den. }\end{array}$ \\
\hline & Karriere-Grants & $\begin{array}{l}\text { Anzahl vergebene Inter- } \\
\text { view Grants } \\
\text { (Output) }\end{array}$ & Zielwert zu definieren & $\begin{array}{l}\text { für den Zeitraum 2011- } \\
2019 \text { wurden durch- } \\
\text { schnittlich } 80 \text { Interview } \\
\text { Grants vergeben }\end{array}$ & $\begin{array}{l}\text { Abhängig } \\
\text { Zielwert }\end{array}$ & $\begin{array}{l}\text { Indikator, inwiefern } \\
\text { ForscherInnen aus } \\
\text { dem Ausland an } \\
\text { F\&E-Unternehmen } \\
\text { herangeführt wur- } \\
\text { den }\end{array}$ \\
\hline $\begin{array}{l}\text { Vernetzung und Inter- } \\
\text { nationalisierung von } \\
\text { Forscherlnnen }\end{array}$ & $\begin{array}{l}\text { Kein Instrument zu- } \\
\text { ordenbar }\end{array}$ & & & & & \\
\hline
\end{tabular}




\section{8 | Schlussfolgerungen}

Das Programm Talente zielt auf die Unterstützung von Menschen in der angewandten Forschung über den gesamten Karriereverlauf und ist aufgrund der sehr weit gefassten Zielgruppe, die potentielle ForscherInnen und damit alle Kinder und Jugendlichen miteinschließt, sehr breit aufgestellt. Es adressiert neuralgische Phasen in der Karriere (z. B. Berufseinstieg) bzw. versucht durch Interventionen diese zu beeinflussen (Bildungsentscheidungen, Berufswahl). Zudem zielt das Programm auf Strukturveränderungen, die den Boden für eine bessere Ausschöpfung des Humanpotentials im anwendungsorientierten, naturwissenschaftlich-technischen FTI-Bereich aufbereiten sollen. Als Humanpotentialprogramm stellt es im FFG-Portfolio nicht nur eine Ausnahme dar, sondern verbessert indirekt und langfristig gesehen über die Unterstützung von Forscherlnnen auch die Wirkung anderer Forschungsförderungsprogramme, in denen diese ForscherInnen ihre Talente in unterschiedlichen Projekten einsetzen. Lessons learnt für die einzelnen Instrumente sind wie folgt:

\section{Praktika für Schülerinnen und Schüler}

Die Praktika für Schülerinnen und Schüler können als erfolgreiches Instrument angesehen werden. Sie tragen zur Steigerung des Interesses der PraktikantInnen an Naturwissenschaft/Technik bei und werden von den befragten ExpertInnen als spannender Einstieg in naturwissenschaftliche und technische Karrieren gesehen. Auch bei den PraktikumsanbieterInnen wird das Engagement in Sachen Nachwuchsförderung positiv beeinflusst. Die Quotenregelung (50\% PraktikantInnen aus AHS, wenn mehrere Praktika angeboten werden) hat dazu geführt, dass nicht nur mehr als die Hälfte der PraktikantInnen aus AHS stammen, sondern auch der Mädchenanteil auf fast $50 \%$ gestiegen ist, und sollte unbedingt beibehalten werden. AHS-SchülerInnen absolvieren zwar wesentlich häufiger ihre Praktika in Forschungseinrichtungen und speziell in Universitäten als in Unternehmen, dies wird jedoch als wenig problematisch eingestuft, da hier die Praktika den Anstoß für die Bildungsentscheidung nach der Schule geben können, und weniger einen direkten Einstieg ins Berufsleben darstellen. Die Programmlinie zeigt neben diesen positiven Wirkungen - trotz ihrer Kleinteiligkeit - auch ein relativ ausgewogenes Verhältnis zwischen eingesetzter Fördersumme und benötigten Ressourcen für die Abwicklung; damit sollte diese Programmlinie weiter einen fixen Platz im Talente-Portfolio einnehmen.

Praktika werden eher von interessierten und engagierten SchülerInnen mit einem unterstützenden sozialen Umfeld in Anspruch genommen. Wenn dieses fehlt, müssten vor allem Lehrkräfte und Schulen aktiv in die Vermarktung der Praktikumsplätze einbezogen werden. Der derzeitige Multiplikatoreffekt der Schulen ist aber gering: nur 4 \% der Schulen nahmen im Jahr 2019 mehr als 10 Praktikumsplätze in Anspruch. Hier besteht also noch ein relativ hohes Potential, das bei erfolgreicher Umsetzung eine entsprechend höhere Dotierung der Programmlinie nach sich ziehen sollte. 
Kontrovers wird die Tatsache gesehen, dass im Rahmen von Talente Pflichtpraktika der BHS gefördert werden. ExpertInnen halten dies für kontraproduktiv, ebenso wie die Tatsache, HTLSchülerInnen zu fördern, die ja schon eine Ausbildungsentscheidung in Richtung Technik gemacht haben. Ein Ausschluss aller Pflichtpraktika würde aber auch Schülerlnnen der nicht-technischen BHS treffen, ein Ausschluss der HTL würde zudem dazu führen, dass das Interesse der Unternehmen an den Praktika stark sinkt und ihnen die Möglichkeit nimmt, im Rahmen geförderter Praktika zukünftige MitarbeiterInnen, die direkt nach der Schule einsetzbar sind, kennenzulernen.

\section{Talente regional}

Auch Talente regional kann als erfolgreich bezeichnet werden, was nicht zuletzt an der steigenden Anzahl von eingereichten Projekten sichtbar wird. Auch zeigt das Review, dass durch den realitätsnahen Einblick in die Tätigkeit von TechnikerInnen und ForscherInnen das Interesse, wenn nicht sogar Begeisterung der Kinder und Jugendlichen für Naturwissenschaft und Technik geweckt wird. Die beteiligten PädagogInnen profitieren vom Kennenlernen neuer pädagogischer Konzepte und innovativer Unterrichtsmethoden und die Schulen von einer Stärkung des Stellenwerts von Naturwissenschaft und Technik sowie von der Verbesserung ihrer technischen Infrastruktur. Auch entstehen nachhaltige regionale Kooperationen zwischen Bildung, Wissenschaft und Wirtschaft. Zu diesen positiven Effekten kommt, dass die Umsetzung der Programmlinie im Verhältnis ausgegebene Fördersumme zu administrativen Kosten als durchaus ausgewogen beurteilt werden kann, insbesondere da in diesem Fall viele Erstfördernehmerlnnen betreut werden.

Dem entgegen stehen ein hoher organisatorischer Aufwand der FördernehmerInnen und das wenig förderliche schulische Setting. Hinzu kommt der Innovationsanspruch im Zusammenspiel mit der Vorgabe nach Geschlechterausgewogenheit und der Integration von Kindern und Jugendlichen unabhängig ihrer sozialen oder geographischen Herkunft. Hier könnte eine stärkere Flexibilisierung des Instruments Abhilfe schaffen. Beispiele dafür sind die Zulassung einer noch stärkeren Fokussierung auf bestimmte Altersstufen (z. B. nur Vorschul- und Volksschulkinder, nur Sekundarstufe I) sowie eine Aufweichung des Innovationsanspruchs dahingehend, dass erprobte Projekte in adaptierter Form in einer anderen Region, mit anderen Unternehmen oder für andere Altersgruppen für eine neuerliche Förderung zugelassen werden. Dies könnte auch der Nachhaltigkeit der Projekte zuträglich sein, weil erprobte Konzepte neuerlich zur Anwendung kommen.

Da das Instrument insgesamt als erfolgreich eingestuft werden kann und die Nachfrage äußerst hoch ist, könnten mit einer jährlichen Ausschreibung mit entsprechender Mittelerhöhung und der damit größeren Zahl an geförderten Projekten mehr Bildungseinrichtungen und Kinder / Jugendliche erreicht werden. Damit könnten zum einen mehr Regionen von den positiven Effekten profitieren und zum anderen eine größere Kontinuität bei den Interventionen sichergestellt werden beides förderliche Faktoren in Hinblick auf strukturelle Veränderungen. 


\section{FEMtech Praktika für Studentinnen}

Die Praktika für Studentinnen sind am „,begehrtesten“ im Rahmen des Talente-Instrumentenportfolios. Sie stärken das Interesse der Studentinnen am Themenfeld und an einer Tätigkeit in Naturwissenschaft und Technik und bieten eine realistische Sicht auf die beruflichen Möglichkeiten. Auch die Fördernehmerlnnen profitieren davon, künftige Mitarbeiterinnen im Berufsalltag kennenzulernen. Zu diesen positiven Effekten kommt, dass FEMtech Praktika für Studentinnen hinsichtlich des Verhältnisses von Fördervolumen zu administrativem Aufwand am effizientesten ist. Hier kommt zum Tragen, dass es sich für die Fördernehmerlnnen um ein einfach zu handhabendes Instrument handelt und sich der Beratungsaufwand in Grenzen hält.

Einziger Wermutstropfen im Zusammenhang mit dieser Programmlinie ist die schnelle Ausschöpfung der Fördermittel und die meist vorzeitige Schließung der Ausschreibung. Hier sind in den meisten Fällen Unternehmen benachteiligt, weil Forschungseinrichtungen meist gleich zu Beginn mehrere Förderungsansuchen für Praktikantinnen stellen. Damit kommt es auch zu einer vermehrten Konzentration der Praktikumsplätze bei einigen Organisationen, meist Forschungseinrichtungen. Damit mehr Organisationen von der Förderung profitieren können, das Instrument breiter gestreut wird und um die Praktikumsstellen in Unternehmen zu forcieren, wird eine Deckelung der geförderten Praktikumsstellen pro Organisation vorgeschlagen. Denkbar sind z. B. 20 bis 30 Praktikumsplätze pro Organisation. Zudem könnte eine Ausschreibung in zwei zeitlich unabhängigen Phasen, wie sie im Jahr 2019 einmal erfolgte, zu einer weiteren Streuung bei den Fördernehmerlnnen führen, womit die Praktika noch besser an den Bedarf der Fördernehmerlnnen angepasst werden könnten.

\section{FEMtech Karriere und FEMtech Karriere-Check für KMU}

Im Rahmen von FEMtech Karriere beschäftigen sich Organisationen gezielt mit dem Thema Gleichstellung und bei den Geförderten kommt es zu einer gestiegenen Akzeptanz und Awareness für das Thema und dessen nachhaltiger Verankerung, einer Veränderung der Unternehmenskultur, zu einer Verbesserung der Arbeitsbedingungen und der Work-Life-Balance sowie zu erfolgreichen Qualifizierungs- und Karriereplanungsaktivitäten von Frauen. Somit werden hier in den geförderten Organisationen faire Rahmenbedingungen für Frauen und Männer geschaffen.

Allerdings wird das Instrument wenig nachgefragt und die Antragstellerlnnen haben meist schon eine erhöhte Sensibilität für die Thematik. Daher bleiben die Wirkungen auf die - wenigen - teilnehmenden Organisationen beschränkt. Diese bescheidenen Wirkungen müssen zudem einem relativ hohen Beratungsaufwand gegenübergestellt werden; die administrativen Kosten entsprechen jedoch in etwa dem Fördervolumen der Programmlinie und diese wird damit von der FFG relativ effizient umgesetzt.

Um wirkliche strukturelle Änderungen der Rahmenbedingungen für mehr Chancengleichheit in Naturwissenschaft und Technik zu schaffen, müssten FEMtech Karriere Projekte in wesentlich mehr Unternehmen und Forschungseinrichtungen durchgeführt werden, um eine kritische Masse an "gegenderten“ Organisationen und in weiterer Folge Spillover-Effekte zu erreichen. Die größte Herausforderung ist daher, mehr Unternehmen und Forschungseinrichtungen für die Programmlinie zu gewinnen. Dafür müssten breite Mobilisierungsmaßnahmen sorgen, die weit über die 
„Talente-Klientel“ hinausgehen und die typischen FFG-Fördernehmerlnnen umfassen. Dies bedeutet aber auch eine entsprechende substantielle Erhöhung der finanziellen Mittel für PR-Maßnahmen und Zielgruppenmobilisierung für ein Instrument, das erst mittel- bis langfristig seine Wirkung entfaltet. Selbst wenn diese Mittel nicht bereitgestellt werden, sollten in jedem Fall Good Practice Beispiele und Erfolgsgeschichten bereits geförderter Projekte allen FFG-Kundlnnen breitenwirksamer zugänglich gemacht werden. Zudem sollten mögliche Synergieeffekte mit der Initiative FEMtech des BMK zur Zielgruppen-Mobilisierung geprüft werden.

Daneben schlagen ExpertInnen eine Namensänderung vor. „FEMtech Karriere“ impliziere, dass das Programm nur Frauen helfen soll, Karriere zu machen. Im Fokus des Instruments steht aber die Veränderung der Unternehmenskultur, damit die Organisation sich für die Zukunft rüstet und im Kampf um Fachkräfte einen Wettbewerbsvorteil erzielt, da es faire und gute Arbeitsbedingungen für alle schafft. Der Name sollte dies widerspiegeln und könnte dafür sorgen, dass die Attraktivität des Instruments steigt. Zudem sollte die Möglichkeit forciert werden, im Rahmen des Projekts einen Gender Equality Plan zu entwickeln, der zunehmend bei (internationalen) Förderprogrammen gefordert werden wird bzw. eine gute Referenz darstellt (z. B. Horizon Europe).

Für NeueinsteigerInnen sind die Anforderungen oft zu komplex, der FEMtech Karriere-Check für KMU als EinsteigerInneninstrument wurde nach zwei Jahren wieder eingestellt, obwohl im zweiten Ausschreibungsjahr die Zielgröße an geförderten Projekten erreicht wurde und auch die meisten Karriere-Check FördernehmerInnen im Anschluss ein Karriere-Projekt durchgeführt haben. Daher sollte eine Neueinführung eines „EinsteigerInneninstruments“ diskutiert werden, um genderunerfahrene Organisationen an das Thema heranzuführen. Da es oftmals sehr lange dauert, bis ein FEMtech Karriere Projekt eingereicht werden kann und bereits ein gewisses Maß an Genderexpertise in der Organisation vorhanden sein muss, um einen Antrag (auch aufgrund der vorgegebenen Modulstruktur) stellen zu können bzw. externe Beratung im Vorfeld dafür benötigt wird, könnte ein niederschwelliges Format genau hier ansetzen. Organisationen, die eine grundsätzliche Bereitschaft zu einem Karriere-Projekt haben, aber nicht das notwendige Know-how mitbringen, könnten mit einer Programmlinie für EinsteigerInnen diese Lücke schließen und sich für ein Karriere-Projekt „fit“ machen.

\section{FEMtech Forschungsprojekte}

FEMtech Forschungsprojekte ist sowohl innerhalb Österreichs als auch international gesehen eine einzigartige Programmlinie und genießt innerhalb der Genderforschungscommunity mittlerweile auch über Österreich hinausgehend ein Ansehen. Es besteht auch hohe Nachfrage: nur etwa ein Fünftel bis ein Viertel der eingereichten Projekte wird gefördert. Mittlerweile müssen aufgrund der hohen Nachfrage auch qualitativ sehr hochwertige Projekte abgelehnt werden. Da sich die Antragstellung aus Sicht der förderwerbenden Organisationen als sehr aufwändig darstellt, ist daher zu überlegen, inwiefern ein zweistufiges Antragsverfahren hier sinnvoll umsetzbar ist. Eine mögliche Benachteiligung von Organisationen mit wenig Antragserfahrung kann aber auch in einem zweistufigen Verfahren nicht ausgeschlossen werden. In jedem Fall würde eine jährliche Ausschreibung und die damit verbundene Mittelausweitung dazu führen, dass mehr Forschungsprojekte mit einem Genderfokus gefördert werden könnten und damit eine gewisse Prä- 
senz des Themas unterstützt wird. Für diese Mittelausweitung spricht auch, dass FEMtech Forschungsprojekte im Verhältnis zur Fördersumme relativ wenig administrative Ressourcen innerhalb des Talente-Teams bindet.

Da die Programmlinie den Anspruch erhebt, durch die Einbindung der Genderdimension auch die Qualität von (zu entwickelnden) Produkten zu erhöhen, sollten die Projektergebnisse und Projektmanagementprozesse in den geförderten Projekten für den Bereich Forschung, Entwicklung und Innovation insgesamt von Interesse sein. Daher kann die aus vorhergehenden Analysen aus den Jahren 2014 (Heckl et al.) und 2016 (Wroblewski) geäußerte Empfehlung einer Forcierung der Disseminationsaktivitäten nochmals wiederholt werden. Um eine breitenwirksamere Vermarktung wirtschaftlich erfolgreicher Projekte und eine Reflexion des Forschungs- und Entwicklungsprozesses sowie strukturelle Veränderungen in Organisationen und der Forschungsund Entwicklungsarbeit zu bewirken, muss die Dissemination über das Programm Talente bzw. die Programmlinie hinausgehen und auch andere Veranstaltungen und thematische Plattformen nützen. Hier wird eine verstärkte Vernetzung mit anderen Programmen in der FFG angeregt, um geeignete Disseminationsmöglichkeiten anzuregen.

Eine andere Möglichkeit wäre, im Rahmen anderer Programme der Forschungsförderung den Bereich Gender stärker zu integrieren, beispielsweise über eine höhere Gewichtung der Genderkriterien im Rahmen von Bewertungsverfahren oder der Reservierung eines Teils der Fördermittel für Projekte mit explizitem Genderfokus in den unterschiedlichen Programmen.

\section{Karriere-Grants}

Insgesamt betrachtet zeigten sich die meisten geförderten Einzelforscherlnnen sehr zufrieden mit der Förderung, und auch die gestiegenen Antragszahlen weisen auf eine entsprechende Nachfrage hin. Allerdings ist die Wirkung der Förderung als äußerst gering einzuschätzen. Der Mitnahmeeffekt ist sehr hoch, nur für einen sehr geringen Anteil der Geförderten ist die Förderung im Rahmen der Relocation Grants ein entscheidender Grund, nach Österreich umzuziehen und auch die Interview Grants scheinen in den meisten Fällen ein "Goodie“ zu sein, da sich hier auch immer mehr Möglichkeiten bieten, Vorstellungsgespräche online oder im Rahmen von Recruiting-Veranstaltungen durchzuführen. Zudem kommt der im Verhältnis zur Fördersumme sehr hohe administrative Aufwand, der darin begründet liegt, dass hier zum einen Einzelpersonen gefördert werden, zum anderen eine Vielzahl an förderbaren Kostenkategorien vorliegen, die eine Prüfung der von den AntragstellerInnen übermittelten Abrechnungsunterlagen nach sich ziehen (Rechnungen von Reisen, Hotels, Umzugsfirma, Deutschkurse etc.). Daher muss das Instrument grundsätzlich in Frage gestellt werden, zumal die Gewinnung von Forscherlnnen aus dem Ausland primär von der Attraktivität des Forschungsstandorts und den Rahmenbedingungen abhängt und ein finanzieller Beitrag für Interview- oder Umzugskosten keine Rolle spielt. Die Maßnahme einzustellen wird auch vor dem Hintergrund der Überzeichnung anderer erfolgreicher Programmlinien innerhalb von Talente empfohlen.

Sollte am Instrument dennoch festgehalten werden, gibt es eine Reihe potentieller Verbesserungsmöglichkeiten bzw. Aspekte der Förderung, die diskussionswürdig erscheinen: Einige FördernehmerInnen bemerken, dass es für sie auf Basis der verfügbaren Informationen nicht klar war, welche Kosten für den Zuschuss anrechenbar waren (z. B. Visa-Kosten). Eine Auflistung 
anhand häufig angerechneter Kosten könnte hier hilfreich sein. Auch bei den anrechenbaren Kosten wünschen sich einige Befragte Änderungen ebenso wie bei den Fördervoraussetzungen. Beispiel ist hier die Voraussetzung eines Abschlusses auf Master-Niveau von Dual Career Antragstellerlnnen. Es stellt sich insbesondere beim Dual Career Grant die Frage, worauf die Förderung abzielt: Soll dadurch der gemeinsame Umzug einer Relocation Grant Fördernehmerin bzw. eines Relocation Grant Fördernehmers unterstützt werden, ist diese Einschränkung nicht nachvollziehbar. Wenn tatsächlich nur hochqualifizierte PartnerInnen von Dual Career Grant gefördert werden, könnten diese auch für einen Relocation Grant in Frage kommen. Für die Entscheidung, ob man gemeinsam mit der Partnerin oder dem Partner umzieht, scheint der Bezug der Förderung ohnehin keine Rolle zu spielen, wie die Antworten aus der Befragung zeigen.

Jedenfalls angedacht werden sollte eine Einschränkung der Zielgruppe des Relocation Grants auf Forscherlnnen in geographisch weiter entfernt liegenden Regionen. Im Zuge dessen sollte dann auch die maximale Förderhöhe angepasst, d. h. erhöht bzw. entsprechend gestaffelt werden. Die Höhe des Zuschusses kann in einigen Fällen ausreichen, die Umzugskosten zur Gänze zu decken, in anderen Fällen (vor allem bei Umzügen aus geographisch weiter entfernten Regionen und bei Umzügen mit der Familie) decken sie nur einen Bruchteil der Kosten. Auch hier sollte nachjustiert und hinterfragt werden, wer die Zielgruppe eigentlich ist und ob eine Staffelung der Zuschusshöhe nicht sinnvoll wäre, z. B. nach Herkunftsregion, Familienstand, Einkommen oder ähnlichen Kriterien.

\section{Die österreichische Jobbörse für Forschung, Entwicklung und Innovation}

Die Jobbörse ist ein Service, das die Suche nach (hoch-)qualifizierten Fachkräften durch Unternehmen, Hochschulen und außeruniversitären Forschungseinrichtungen und die Suche nach Stellen im Bereich Forschung, Technologie und Innovation durch Fachkräfte erleichtern soll. Der angestrebte Zielwert an BesucherInnen konnte nicht erreicht werden, die Zunahme an Ausschreibungen im Beobachtungszeitraum deutet aber auf eine gestiegene Nutzung durch Organisationen hin. Wichtig ist hierbei eine möglichst umfangreiche Abdeckung aller veröffentlichten Stellen in diesem Bereich in Österreich und eine möglichst gute Reichweite der FFG Jobbörse. Sowohl die Abdeckung als auch die Reichweite können nicht abschließend bewertet werden, da hierzu Vergleichsdaten fehlen. Da die Jobbörse neben den Websites der Organisationen auch Jobportale durchsucht (sofern sich frei zugänglich sind), kann von einer guten Abdeckung ausgegangen werden.

Im Rahmen dieser Evaluierung konnte auf Basis der verfügbaren Informationen auch nicht abschließend geklärt werden, ob die Jobbörse sowohl für Suchende als auch Jobanbieterlnnen einen Zusatznutzen bringt, der von anderen Dienstleistungsangeboten nicht bereits abgedeckt wird. Es wird daher empfohlen, mittels UserInnenbefragung zu eruieren, ob die Jobbörse einen entsprechenden Nutzen entfaltet. Dazu könnten zum einen die registrierten Unternehmen befragt werden, deren Websites regelmäßig nach Ausschreibungen gescreent werden, sowie die BesucherInnen der Jobbörse nach Nutzung der Seite, beispielsweise mittels einer Onsite-Befragung. 


\section{Begleitmaßnahmen}

Im Förderschwerpunkt werden Begleitmaßnahmen umgesetzt. Im Rahmen der Prämierungsveranstaltungen in der Programmlinie SchülerInnenpraktika werden die 20 SchülerInnen mit den besten Praktikum-Reports prämiert. Somit können besonders engagierte PraktikantInnen ausgezeichnet werden. Die Fotos der Prämierungsfeier sind auf der FFG-Website verfügbar. Die Praktikum-Reports könnten - wenn der Veröffentlichung zugestimmt wird - ebenfalls online gestellt werden, um den Interessierten (SchülerInnen wie Organisationen) zu zeigen, wie vielfältig solche Praktika ausgestaltet sein können. Diese könnten auch zur Informationsstreuung und Kundlnnenakquise genützt werden.

Die Netzwerkveranstaltungen im Rahmen von Talente regional, die gut angenommen werden, dienen der Vorstellung der Förderung und dem Erfahrungsaustausch, es werden aber auch Impulsvorträge gehalten. Zur Stärkung des Formats wird empfohlen, die Vorstellung von Good Practice Beispielen und die Möglichkeit, sich mit erfahrenen Fördernehmerlnnen auszutauschen, noch stärker in den Fokus der Veranstaltung zu rücken. Eine weitere Möglichkeit des Erfahrungsaustauschs ist ein Online-Format, um eventuell regional verteilt zusätzlichen Organisationen eine Teilnahme zu ermöglichen.

Die bisher zwei Infoveranstaltungen für FEMtech Forschungsprojekte hatten regen Zulauf, obwohl hier kaum EinsteigerInnen gefördert werden, was für das hohe Informationsbedürfnis der potentiellen FördernehmerInnen spricht. Corona-bedingt fand die Infoveranstaltung zur 7. Ausschreibung online statt. Es wird angeregt, ein solches Online-Format weiterhin (zusätzlich) anzubieten, um einerseits mehr InteressentInnen die Möglichkeit zu bieten, auf diesem Wege Informationen zu sammeln und andererseits mehr FEMtech Forschungsprojekte vorstellen zu können, da diese am besten illustrieren, wie ein Genderfokus die Forschung bereichern kann.

Die FEMtech Netzwerktreffen werden veranstaltet, um über Neuigkeiten aus dem Förderschwerpunkt zu informieren und zeigen aktuelle FEMtech Themen auf. Es gibt die Möglichkeit zum informellen Austausch und zur Förderberatung durch die FFG. Allerdings nehmen vorwiegend Personen an den Treffen teil, die sich mit Chancengleichheit in Forschung, Naturwissenschaft und Technik beschäftigen. Damit kann keine breite Streuung der Thematik in die Forschungscommunity erwartet werden. Um das Thema Chancengleichheit in der Forschung auf die allgemeine Agenda zu setzen und zur breiteren Mobilisierung von AntragstellerInnen kann über eine Öffnung des Formats, von der Einladungspolitik bis zur Streuung der Dokumentation, versucht werden, den InteressentInnenkreis zu erweitern. Dann könnten die Treffen auch besser zur Dissemination der Information über die Förderformate, insbesondere über FEMtech Karriere, genützt werden. 


\section{Gesamtfazit}

Auf der Grundlage der detaillierten Betrachtung der einzelnen Programmlinien sowie des Gesamtprogramms lassen sich auch Schlussfolgerungen für das Programm als Ganzes ziehen. Die Breite des Programms ist ein wichtiges Charakteristikum, birgt aber die Gefahr der Zielüberfrachtung sowie einer mangelnden Fokussierung vor dem Hintergrund begrenzter Ressourcen. Insbesondere das Interventionsfeld "Talente finden" bedarf aus Sicht des Evaluierungsteams einer kritischen Analyse. So scheinen die Instrumente nur teilweise zur Zielerreichung geeignet und die Wirkungen sind äußerst gering bzw. nicht umfassend zu beurteilen. Es wird daher empfohlen, dieses Interventionsfeld grundlegend zu hinterfragen und zu diskutieren, ob entweder Ziele und dazu passende Instrumente neu aufgesetzt werden oder aber das Interventionsfeld zugunsten der anderen aufgegeben wird und sich Talente auf die Themen Nachwuchsförderung und Chancengleichheit fokussiert. Mit diesem Fokus könnten die Ressourcen konzentrierter eingesetzt werden, das Programm müsste allerdings Abstriche in Hinblick auf seinen umfassenden Ansatz machen. Die Jobbörse könnte bei einer etwaigen Aufgabe der Karriere-Grants und des Interventionsfelds "Talente finden“ als unterstützendes Instrument begriffen und entsprechend vermarktet werden.

Essentiell für ein "nicht-klassisches“ Forschungsförderungsprogramm ist die Mobilisierung der Zielgruppen. Zum einen sollen in hohem Maße FFG-EinsteigerInnen für das Programm gewonnen werden, zum anderen gilt es, typische FFG-Klientel für Instrumente zu gewinnen, die eher mittel- bis langfristige Wirkungen entfalten und oftmals nicht direkt sichtbar sind. Der Informationsstreuung kommt daher große Bedeutung zu. Dies spiegelt sich zurzeit nicht in den administrativen Kosten für Mobilisierung und Veranstaltungen wider, die nur einen Bruchteil des administrativen Budgets beanspruchen. Zur Förderung der Breitenwirksamkeit des Programms sollten daher die Mobilisierungsmaßnahmen ausgeweitet werden.

Der Breitenwirksamkeit steht auch entgegen, dass das Programm in seiner Gesamtheit und unter dem Namen „Talente“ noch immer nicht als solches bekannt ist, im Gegensatz zu den einzelnen Programmlinien bzw. Interventionsfeldern (z. B. FEMtech), die für viele Fördernehmerlnnen und für die meisten Expertinnen und Experten ein Begriff sind. Hier steht zur Diskussion, das „Branding“ zur verstärken, wobei die Bezeichnung der Programmlinien den Programmnamen enthalten sollten. Auch ist bei der Namensgebung der Programmlinien darauf Bedacht zu nehmen, dass dieser die Förderung adäquat beschreibt (siehe FEMtech Karriere). Wenn das Programm zur Marke wird und an Bekanntheit gewinnt, könnte dies zur Unterstützung seiner Mission, der Entwicklung von Humanpotential für die angewandte Forschung, beitragen. Das Rebranding des gesamten Programms, durch das die Zugehörigkeit aller Programmlinien zum Programm deutlich sichtbar wird, sollte durch einen öffentlichkeitswirksamen Relaunch begleitet werden.

Abschließend muss festgehalten werden, dass ein Programm wie Talente, das an den Strukturen und Rahmenbedingungen ansetzt und nichts weniger als Veränderungen von tradierten Einstellungen und Rollen bewirken will, bei begrenzten Mitteln immer nur einen kleinen Beitrag leisten kann. Die Wichtigkeit der Förderung des Humanpotentials muss auch innerhalb der Forschungscommunity wirklich „ankommen“ und mehr als ein Lippenbekenntnis darstellen. Denn die Zahlen 
sind v. a. in Hinblick auf die Repräsentanz von Frauen im Forschungssektor - und hier insbesondere im industriellen Sektor - nach wie vor ernüchternd. Die She Figures 2018 (European Commission 2019) zeigen, dass in Österreich nur rd. 17 \% der ForscherInnen in Unternehmen Frauen sind. Und auch aus dem Wirkungsmonitoring der FFG Förderungen 2019 (Nindl / Kaufmann 2020) ist ersichtlich, dass Frauen in FFG-geförderten Projekten weiterhin unterrepräsentiert sind und der Anteil der Projektleiterinnen bei rd. $10 \%$ liegt und zuletzt sogar gesunken ist. Daher bedarf es einer Aufwertung und eines Mainstreaming des Themas innerhalb der FFG und des BMK. Die Themen Humanressourcen und Chancengleichheit müssen als Querschnittsthemen begriffen werden und entsprechende Berücksichtigung in den anderen Programmen finden sowie deren Informationskanäle auch für das Programm Talente genützt werden. 


\section{9 | Anhang}

\section{1 | Fallstudien FEMtech Karriere}

\subsection{1 | Fallstudie Akaryon $\mathrm{GmbH}$}

\section{Organisation}

Die Akaryon Gmbh ist seit 1999 im Bereich Unternehmensberatung und Informationstechnologie tätig und bietet Webtools schwerpunktmäßig im Bereich Umweltinformatik an. D.h. es werden Softwareangebote und Tools entwickelt, die dabei helfen, Nachhaltigkeit möglichst einfach in den Unternehmensalltag zu integrieren, wie z. B. Energiewende-Rechner, $\mathrm{CO}_{2}$-Fußabdruckrechner, CSR-Management- und Nachhaltigkeits-Reporting-Tools. Außerdem bietet Akaryon angewandte Nachhaltigkeitsforschung an, um umweltfreundliches Handeln zu fördern und unterstützt Unternehmen, NGOs, Plattformen und diverse Institutionen bei der Abwicklung von Projektförderungen.

Bei Akaryon sind 14 MitarbeiterInnen (September 2020) tätig, wobei das Geschlechterverhältnis ziemlich ausgeglichen ist. Die Arbeitssituation im Unternehmen ist insofern ungewöhnlich, weil zu $100 \%$ im Home-Office gearbeitet wird. Die MitarbeiterInnen kommen nur wenige Male im Jahr zu Firmenmeetings oder Kundlnnenterminen persönlich zusammen. Um einen Überblick über die Aufgaben und Tätigkeitsbereiche zu bewahren, wird ein firmeninternes Ticketsystem eingesetzt. Im Unternehmen gibt es sehr flache Hierarchien, dennoch sind die Verantwortlichkeiten klar geregelt. Die MitarbeiterInnen schätzen die flexiblen Arbeitszeiten, die es Ihnen ermöglichen, sich noch anderen Tätigkeiten, wie Betreuungs- und Pflegeaufgaben, Aus- und Weiterbildungsaktivitäten etc., zu widmen. Sie erleben ihre Arbeit als sehr effizient und empfinden den hohen Dokumentationsgrad aufgrund der vorwiegend schriftlichen Kommentierung als Vorteil.

\section{Ziele und Motivation}

Akaryon hat sich im Jahr 2016 an den FFG Ausschreibungen für den FEMtech Karriere-Check für KMU sowie im Anschluss für ein FEMtech Karriere Projekt beteiligt. Das Unternehmen hatte zuvor gewisse Berührungspunkte zum Gender-Thema und war mit den Förderangeboten der FFG sehr vertraut. Anstoß für eine Beteiligung war der Wunsch, sich umfassender und konzentrierter mit Gender, Gleichstellung und Chancengleichheit auf Ebene der Geschäftsführung und der Mitarbeiterlnnen zu befassen und eine systematischere und gendersensible Weiterbildung und Karriereplanung zu implementieren.

Neben der Durchführung einer Genderanalyse der Arbeitsprozesse im Unternehmen im Rahmen des Karriere-Checks standen darauf aufbauend die aktive Verankerung von Gender-Aspekten, Frauenförderung, Chancengleichheit und Gleichstellung im Unternehmen sowie die entsprechende Positionierung von Akaryon als nachhaltiges, gendersensibles, die Karriereplanung gezielt unterstützendes Unternehmen im Rahmen des FEMtech Karriere-Projekts im Vordergrund. 


\section{Projektinhalte}

Das Projekt „Karriere-Check@akaryon“ wurde im Zeitraum 07/2017 bis 04/2018 umgesetzt. Dabei wurde eine umfassende Genderanalyse des Unternehmens erstellt sowie Entwicklungspotentiale und Maßnahmenempfehlungen zur Verbesserung der Chancengleichheit, zur Gleichstellung von Frauen und Männern und zur Erhöhung des Frauenanteils in Führungspositionen abgeleitet. Für Akaryon stellte die Erstanalyse im Rahmen des FEMtech Karriere-Checks für KMU eine wichtige Grundlage für das FEMtech Karriere-Projekt dar. Diese grundlegende Analysephase wird als unabdingbare Voraussetzung für weitere Aktivitäten gesehen und hätte sonst in das FEMtech Karriere-Projekt integriert werden müssen.

Auf der Analyse der Gendersituation im Unternehmen aufbauend wurde das FEMtech-Karriere Projekt „fair-career@akaryon an der Schnittstelle zwischen IT und Nachhaltigkeit“ im Zeitraum 06/2019 bis 03/2020 durchgeführt. Zum Wissens- und Kompetenzaufbau im Bereich Gender standen Workshops und Trainings im Vordergrund. Zur Karriereentwicklung wurde ein bedarfsorientierter Weiterbildungsplan für alle MitarbeiterInnen erstellt und zusätzlich zu den jährlich stattfindenden MitarbeiterInnen-Gespräche eine systematischere und gendersensible Karriereplanung implementiert. Auch die Entwicklung diverser Arbeitstools, wie Guidelines für MitarbeiterInnen-Gespräche, Gender- und Diversity-Guidelines, Start- und Wiedereinstiegspläne für neue bzw. karenzierte MitarbeiterInnen, und gendersensibler Stellenausschreibungen für Forschung und Innovation erfolgte im Zuge des Projekts. Darüber hinaus wurde im Rahmen einer neuen Kommunikationsstrategie die Präsentation des Unternehmens durch Kompetenz-Visitenkarten des Unternehmens und die Überarbeitung der Website weiter optimiert.

Ein Projektschwerpunkt stellte die vergleichende Analyse von Monitoring-Systemen zu Chancengleichheit und Frauenförderung dar. Dazu wurde eine Studie erstellt und die Erkenntnisse im Rahmen des Nachhaltigkeitsreporting-Tools „ESG-Cockpit“ als ein eigenes Modul "Chancengerechtigkeit und Frauenförderung" eingebaut. Als zweiter Projektschwerpunkt stand das Thema Home-Office aufgrund der 20-jährigen Erfahrung in diesem Bereich im Fokus. Die Ergebnisse aus dieser Analyse wurden im Rahmen der Studie „Digitale Arbeitswelten \& Chancengerechtigkeit aus Sicht eines Technologieunternehmens" zusammengeführt.

\section{Ergebnisse und Wirkungen}

Das FEMtech Karriere-Projekt ermöglichte es Akaryon, konzentriert am Gender-Thema zu arbeiten. Damit konnten sowohl intern als auch extern positive Effekte erzielt werden. Intern wurde das Selbstbewusstsein der MitarbeiterInnen durch klarere Rollen und persönliche Karrierebilder gestärkt und die Teamidentität gesteigert. Die MitarbeiterInnen nutzen weiterhin die entwickelten Produkte, wie die Gender-Leitfäden, und sie haben eine durchgängige Linie für gendergerechte Formulierungen entwickelt. Ebenso wurden Weiterbildungspläne, -aktivitäten und Karriereplanungen in den regulären Arbeitsalltag integriert. 
Extern wird Akaryon nun verstärkt als Vorreiterbetrieb wahrgenommen, was sich in zahlreichen Einladungen zu Vorträgen, Podiumsdiskussionen, Medienberichten und Einladungen zu Projektteilnahmen, zeigte. Das Unternehmen stand sogar im Finale des Wert!Geschätzt-Wettbewerbs. ${ }^{17}$ Außerdem konnten mit Hilfe der Projektaktivitäten fünf neue MitarbeiterInnen ins Team aufgenommen werden, wenngleich noch bedauert wird, dass bisher keine neue weibliche Programmiererin gefunden werden konnte.

\section{Lessons learnt und Ausblick}

Die Förderung ermöglichte Akaryon, sich intensiver mit dem Gender-Thema auseinanderzusetzen. Ohne finanzielle Unterstützung wäre es nicht möglich gewesen, so umfangreiche Projektaktivitäten zu setzen und die notwendigen Zeitressourcen aufzuwenden. Das Thema bleibt bei Akaryon weiterhin auf der Agenda und die implementierten Aktivitäten werden fortgeführt. Die gendergerechte Kommunikation kommt im regulären Kundlnnenkontakt zum Einsatz, wird aber individuell entsprechend der Kundlnnenpräferenzen angepasst. Akayron ist sich in diesem Zusammenhang seiner Vorreiterrolle auf diesem Gebiet bewusst, und versucht sowohl im Kundlnnenkontakt als auch bei der Öffentlichkeitsarbeit, Bewusstsein für das Gender-Thema zu schaffen. Auch bei Produktentwicklungen und Programmierungen werden Genderaspekte stets mitbeachtet. Das Unternehmen ist in seiner täglichen Arbeit bemüht, dieses Thema weiter in die Öffentlichkeit zu tragen.

Akaryon kann sich auch vorstellen, mittelfristig in rd. zwei bis drei Jahren ein weiteres FEMtech Karriere Projekt einzureichen, damit das Gender-Thema weiterhin auf der Tagesordnung bleibt und neue, größere Vorhaben in diesem Bereich realisiert werden können.

\subsection{2 | Fallstudie BEST - Bioenergy and Sustainable Technologies GmbH}

\section{Organisation}

BEST - Bioenergy and Sustainable Technologies GmbH ist ein K1-Kompetenzzentrum des COMET-Programms, das 2003 als Austrian Bioenergy Centre GmbH (ABC) gegründet wurde. Das Kompetenzzentrum ist in der Forschung, Entwicklung und Demonstration im Bereich der thermischen und biotechnologischen Nutzung von Biomasse, der biobasierten Ökonomie und zukunftsfähigen Energiesystemen tätig. Der Hauptsitz des Kompetenzzentrums ist in Graz, je ein Standort für spezielle Forschungsaufgaben befindet sich in Wien und in Wieselburg, zwei weitere Forschungsstätten sind in Wien-Simmering und Tulln angesiedelt. Organisatorisch ist BEST in fünf Areas, die verschiedene Kompetenzbereiche abbilden, organisiert.

Bei BEST arbeiten mit Stand Ende Juni 202095 Personen, davon 29 Frauen und 66 Männer. Der Anteil an Forscherinnen liegt mit 24 \% über dem Durchschnitt der Bioenergiebranche. Das Arbeits- und Gesprächsklima im Kompetenzzentrum wird als sehr offen und angenehm erlebt.

\footnotetext{
${ }^{17}$ Es handelt sich um einen Landespreis für familienfreundlichste Betriebe der Steiermark: https://www.sfg.at/gruendenund-starten/familienfreundlichste-betriebe
} 
Das Personal konnte die Rahmenbedingungen seiner Arbeit in den letzten Jahren aktiv mitgestalten. Neben einem Gleitzeitmodell gibt es auch die Möglichkeit, im Home-Office zu arbeiten. Die Forschungsarbeiten, die sich teilweise über mehrere Jahre erstrecken, lassen in bestimmten Bereichen eine zeitliche Flexibilität der MitarbeiterInnen zu, bei experimentellen Arbeiten in den Versuchshallen ist jedoch eine gewisse Anwesenheit erforderlich. Das Kompetenzzentrum unterstützt das familiäre Zusammenleben, indem es die Work-Life-Balance gezielt fördert.

\section{Ziele und Motivation}

Seit der Gründung des Zentrums werden Maßnahmen gesetzt, um die Gleichstellung der MitarbeiterInnen zu fördern. Es wurde bereits in den Jahren 2007/2008 ein FEMtech fFORTE Projekt durchgeführt und im Rahmen der FFG Programme „FEMtech Praktika für Studentinnen“ und „FEMtech Karrierewege" wurden in den letzten 10 Jahren insgesamt 78 Praktika für Studentinnen abgewickelt. BEST hat sich bei FEMtech Karriere mit drei Projekten in den Jahren 2015, 2016 und 2019 erfolgreich beteiligt.

Das erste FEMtech Karriere Projekt „Gendergizing - Energizing Gender Mainstreaming in Research and Technology" wurde von 04/2016 bis 03/2017 umgesetzt. Ziel des Projekts war, die Gleichstellung von Frauen und Männern in Forschung und Technik zu fördern und Gender Mainstreaming in der standortübergreifenden Unternehmensstrategie von BEST zu verankern.

Von 04/2018 bis 03/2019 folgte das FEMtech Karriere Projekt „FeMentoring - Mentoring für Frauen in Forschung und Technologie“. Dieses zielte darauf ab, junge Forscherinnen zu fördern und sie auf zukünftige Führungspositionen vorzubereiten. Auch die Genderkompetenz im Unternehmen auszubauen und nachhaltig zu verankern waren Zielsetzungen dieses Projekts.

Das von 03/2020 bis 10/2021 laufende Projekt „Equality Advanced - Gleichstellung in Unternehmensstrukturen und Prozessen abbilden" widmet sich gezielt der mittel- bis langfristigen Erhöhung des Frauenanteils in Führungspositionen. Aufgrund der Einschränkungen in Zusammenhang mit der Corona-Pandemie wurde die Laufzeit kostenneutral um ein halbes Jahr verlängert, um die Aktivitäten nicht nur online, sondern auch in physischer Präsenz durchführen zu können.

\section{Projektinhalte}

Im Rahmen des ersten FEMtech Karriere Projekts „Gendergizing“ stand der Aufbau von Genderkompetenz bei Führungskräften und Stabsstellen sowie die Installierung einer unternehmensinternen Gleichstellungsbeauftragen im Vordergrund. Die Gleichstellung von Frauen und Männern im beruflichen Alltag wurde durch die Entwicklung von Karrieremodellen inklusive transparentem Gehaltsschema und die Integration von Gleichstellungsgrundsätzen in der Betriebsvereinbarung gefördert. Zur Unterstützung der Karriereentwicklung wurden Leitfäden für Mitarbeiterlnnen- und Entwicklungsgespräche sowie Weiterbildungskonzepte erarbeitet und diese in die jährliche Routine integriert. Um die Leistungen von Frauen in Forschung, Technologie und Innovation sowie die erfolgreiche Vereinbarkeit von Beruf und Familie bei BEST sichtbar zu machen, wurden Role Models auf der Homepage sowie in einer Imagebroschüre dargestellt. Auch eine Intranet-Seite zum Thema Gleichstellung wurde eingerichtet, die laufend über Aktivitäten in diesem Bereich informiert. 
Im zweiten FEMtech Karriere Projekt „FeMentoring“ wurde die Genderkompetenz im Unternehmen durch gezielte Schulungsmaßnahmen von Führungskräften und der Gleichstellungsbeauftragten erweitert und nachhaltig in das Unternehmen integriert. Außerdem wurde ein zentrumsweites Mentoring-Programm für wissenschaftliche Mitarbeiterinnen konzipiert und als verbindliche Personalentwicklungsmaßnahme zur Karriereförderung aufgenommen. Im Anschluss an die Projektlaufzeit erfolgte der erste Mentoring-Zyklus mit weiblichen Senior Researchern. Darüber hinaus wurde ein Einführungskonzept für neue MitarbeiterInnen mit entsprechenden Unterlagen erarbeitet, eine Checkliste für gendergerechte Stellenausschreibungen und ein Online-Bewerbungstool für effizientere und anonymisierte Bewerbungsprozesse entworfen.

Im Rahmen des aktuell laufenden FEMtech Karriere Projekts „Equality Advanced“ wird zur Erhöhung des Frauenanteils in Führungspositionen eine umfassende Detailanalyse der Situation im Zentrum mittels der 4 R-Methode (Repräsentation, Ressourcen, Realität und Rechte von Frauen und Männern) in den einzelnen Abteilungen erarbeitet. Auf dieser umfassenden Analyse aufbauend und durch entsprechende Sensibilisierung der Führungskräfte im Rahmen von Workshops werden konkrete Maßnahmen auf Prozessebene abgeleitet und umgesetzt. Darüber hinaus wird ein umfangreiches Work-Life-Konzept entwickelt, das unterschiedliche Lebens- und Arbeitsweisen adressiert, sowie ein Gesundheitskonzept entworfen, um nachhaltige Schritte in der betrieblichen Gesundheitsförderung zu setzen.

\section{Ergebnisse und Wirkungen}

Im Rahmen des ersten FEMtech Karriere Projekts "Gendergizing“ wurde als besonders nachhaltige Maßnahme eine Gleichstellungsbeauftragte installiert. Damit wird das Gender-Thema präsent gehalten, da sie sich explizit dafür verantwortlich fühlt und sich sehr engagiert für Gleichstellungsfragen einsetzt. Die Projekte ermöglichten ihr den vertieften Erwerb von Kompetenzen in diesem Bereich, wodurch ihre Position weiter gefestigt und in weiterer Folge das Bewusstsein vieler MitarbeiterInnen für diese Thematik geschärft wurde. Wenn nun bei Projektanträgen Fragen zur Gleichstellung und Gender-Diversität zu beantworten sind, wird die Gleichstellungsbeauftragte aktiv miteingebunden. Auch bei Stellenausschreibungen wird verstärkt auf gendersensible Formulierungen geachtet, um gezielt Frauen anzusprechen. Dies hat eine größere Anzahl an Bewerbungen von Frauen und die Anstellung von jungen Forscherinnen zur Folge.

Ein weiteres nachhaltiges Instrument ist das Karrieremodell mit dem transparenten Gehaltsschema, das bei den Mitarbeiterlnnen auf sehr positive Resonanz gestoßen ist. Gehaltsunterschiede und -erhöhungen sind dank eines Punkteschemas für alle nachvollziehbar. Dadurch ist die Zufriedenheit mit den Karriereoptionen und finanziellen Möglichkeiten gestiegen, da im Rahmen der MitarbeiterInnengespräche konkret festgelegt wird, wie Gehaltserhöhungen erreicht werden können. Auch die bereits entwickelten Produkte, wie die Gesprächsleitfäden, die Mappe zur Einführung neuer MitarbeiterInnen oder das Online-Bewerbungstool, sind weiterhin laufend im Einsatz und werden als sehr hilfreiche Unterstützung erlebt. Für das Mentoring-Programm, das im Rahmen des zweiten FEMtech Karriere Projekts entwickelt und erprobt wurde, werden breitere Einsatzmöglichkeiten überlegt, damit Frauen und Männer gleichermaßen davon profitieren können. Auch die Möglichkeit, mit den Führungskräften in Workshops gezielt am Gender-Thema weiter zu arbeiten, wird als wichtige und nachhaltige Maßnahme gesehen. Daher ist im Rahmen 
des aktuellen Projekts wieder eine Workshop-Reihe für Führungskräfte mit starken reflexiven Elementen vorgesehen, die in Präsenzform im April/Mai 2021 abgehalten werden soll.

\section{Lessons learnt}

Die FEMtech Karriere Projekte haben geholfen, das Gender-Thema bei BEST nachhaltig zu verankern und die Gleichstellung im Unternehmen weiter voranzutreiben. Insbesondere die Etablierung der Position der Gleichstellungsbeauftragten und deren Engagement stell kontinuierliche weitere Aktivitäten in diesem Bereich sicher. Ohne die Förderungen hätten nicht so umfangreiche Projektaktivitäten im Forschungszentrum realisiert werden können, da die Ressourcen dafür nicht verfügbar gewesen wären. Auch eine Beteiligung an weiteren FEMtech Karriere Projekten wird nach einer gewissen Pause begrüßt. Nach einer Evaluierung der laufenden Aktivitäten könnten Ansatzpunkte für weitere Maßnahmen erhoben werden. Generell wird der Umgang mit dem Gender-Thema und die Förderung der Gleichstellung von Frauen und Männern als Prozess gesehen, der auch nach Abschluss der Projekte weiterfortgesetzt werden muss. Dafür ist jedoch nicht nur das Engagement des Forschungszentrums ausschlaggebend, sondern auch allgemeine gesellschaftliche Rahmenbedingungen, wie die Regelung der Betreuungsaufgaben. Gerade in ländlicheren Regionen, wie am Standort Wieselburg, stellt die Kinderbetreuung am Nachmittag nämlich oft noch eine Herausforderung dar.

\subsection{3 | Fallstudie - Güssing Energy Technologies (GET)}

\section{Zur Organisation}

Güssing Energy Technologies (GET) ist ein unabhängiges Non-Profit Forschungsinstitut, das Grundlagen- und Auftragsforschung zur Nutzung erneuerbarer Energieträger und der Entwicklung neuer Prozesse, Verfahren und innovativer Systemkombinationen betreibt. Die Forschungsbereiche konzentrieren sich auf das thermische Kühlen, den effektiven Einsatz von Fernwärme, biologische Treibstoffe, die Analyse von Kraft-Wärme-Kopplungen sowie von Haustechniksystemen. Mit seinen umsetzungsorientierten Forschungsaktivitäten fungiert Güssing Energy Technologies als Schnittstelle zwischen Wissenschaft und Privatwirtschaft. An Dienstleistungen werden die Unterstützung bei der Erstellung von Energiekonzepten für Industrien und Gemeinden und beim Industrieanlagen- und Siedlungswasserbau angeboten. Darüber hinaus ist das Institut um die Dissemination seines Wissens im Zuge von Forschungsprojekten bzw. Weiterbildungsinitiativen des Clusters Güssing bemüht. Zwei der elf MitarbeiterInnen (zehn Männer, eine Frau) sind auch als Vortragende an der Technischen Universität Wien sowie an Fachhochschulen im Burgenland und in Salzburg tätig.

Um mehr weibliche Arbeitskräfte zu gewinnen, werden gezielt Praktikantinnen gesucht und in die Forschungsaktivitäten eingebunden. Der Arbeitsort Güssing ist als strukturschwache Region jedoch für Nachwuchskräfte weniger attraktiv, auch wenn das Institut über angenehme Rahmenbedingungen, wie flexible Arbeitszeiten und ein transparentes Gehaltsschema verfügt. Auch die Work-Life Balance wird von den Mitarbeiterlnnen gut bewertet. 


\section{Ziele und Motivation}

Das Forschungsinstitut hat den Antrag zu einem FEMtech Karriere Projekt eingebracht, um endlich von langer Hand geplante Tätigkeiten zur Verbesserung der Gleichstellung durchführen zu können. Teilziele davon waren es kurz- bis mittelfristig den Frauenanteil im Unternehmen zu erhöhen, Gender Mainstreaming nachhaltig zu verankern und eine Vorreiterrolle in diesem Bereich im Rahmen seiner Netzwerke (z. B. ACR) einzunehmen.

Aufgrund des hohen Beratungsbedarfs bei der Antragverfassung für das FEMtech Karriere Projekt hat die FFG empfohlen, zuerst den FEMtech Karriere-Check für KMU durchzuführen. Zielsetzung der Genderanalyse war es, eine vertiefte Auseinandersetzung mit dem Thema Chancengleichheit zu ermöglichen und das Genderwissen im Institut professionell aufzubauen. So konnte denn auch in der Folge ein Antrag für FEMtech Karriere verfasst werden. Im Zuge des nachfolgenden FEMtech Karriere Projekts galt es, die Genderkompetenz zu erweitern, sich als attraktiver Arbeitgeber und Arbeitsort für ForscherInnen zu positionieren und den Bereich Diversityforschung innerhalb des Instituts zu stärken.

\section{Projektinhalte}

Um mehr Gender Know-how zu generieren, wurde zunächst der GET Gender Check im Zeitraum von 02/2016 bis 01/2017 durchgeführt. Auf Basis einer Befragung aller Mitarbeiterlnnen und einer umfangreichen Dokumentanalyse wurden im Rahmen von Workshops konkrete Maßnahmen zur stärkeren Verankerung von Gender Mainstreaming definiert, die im anschließenden FEMtech Karriere Projekt aufgegriffen werden sollten. Diese betrafen die stärkere Berücksichtigung der Genderperspektive in Projekten, die Integration einer gendergerechten Sprache sowie die Optimierung des Leitbilds und des Außenauftritts der Organisation nach gendersensiblen Kriterien. Der FEMtech Karriere-Check für KMU stellte für Güssing Energy Technologies eine wichtige, richtungsweisende Hilfestellung für den folgenden Projektantrag dar. Außerdem wurde auf Basis dieses Projekts eine Gender- und Diversitybeauftragte installiert, die nachhaltig als Ansprechpartnerin für genderrelevante Themen fungiert.

Das nachfolgende FEMtech Karriere Projekt fand von 05/2017 bis 05/2018 statt. Zu Beginn des GET Gender II Projekts erfolgte der Kompetenzaufbau im Rahmen von Workshops. Das Wissen aller Mitarbeiterlnnen wurde durch blended learning erweitert und eine gemeinsame Lernplattform als Wissensbasis aufgebaut (die Trainingsplattform https://train.get.ac.at/ ist noch immer in Betrieb und wird seitdem zu $100 \%$ aus Eigenmitteln des Instituts finanziert). Die Genderbeauftragte konnte mit Hilfe von Coaching-Aktivitäten ihre Rolle als Genderbeauftragte stärken und ihre Kompetenzen in diesem Bereich erweitern. Um Gender Mainstreaming dauerhaft in die Firmenphilosophie zu verankern, wurde gemeinsam mit allen Mitarbeiterlnnen im Rahmen eines Workshops ein gendersensibles Leitbild entwickelt. Auch das Thema gendergerechte Sprache wurde aufgearbeitet und die Entscheidung für eine einheitliche Variante am Institut getroffen.

Darüber hinaus erfolgte die Überarbeitung des Entwurfes einer neuen Homepage (2020 musste aus technischen Gründen leider wieder die vorherige Version online gestellt werden) und der PRMaterialien (wie z. B. Firmenbroschüren) in Bezug auf Gendersensibilität und die weitere Optimierung des Außenauftritts. Um den Umgang mit dem Thema zu erleichtern, wurden Check- 
Listen bzw. Richtlinien für eine gendersensible Mediengestaltung und die Berücksichtigung von Gender Mainstreaming in Projekten erstellt. Die Integration der Genderthematik in die technischen Forschungsprojekte wurde als herausfordernd wahrgenommen und anhand von konkreten Praxisbeispielen zu übersetzen versucht. Die entwickelten Check-Listen helfen nun dabei, Genderthemen bei Forschungsprojekten zu berücksichtigen und sie automatisch in den Projektentstehungsprozessen mitzudenken. Seitdem sind die dafür notwendigen Tätigkeiten bei neuen Projektplänen fixer Bestandteil des Arbeitspaketes Projektmanagement.

Schließlich erfolgte eine umfassende Analyse der Vortragstätigkeiten eines Mitarbeiters. Sämtliche Unterlagen und Skripten für die Fachhochschule wurden in Hinblick auf Gendersensibilität analysiert und in Bezug auf gendergerechte Sprache angepasst. Somit konnte Gender auch in der Lehre stärker und nachhaltig etabliert werden. Die Tätigkeiten an den Fachhochschulen werden von den MitarbeiterInnen auch gezielt genützt, um Studentinnen als Praktikantinnen anzuwerben, um so eventuell weitere weibliche Forschungskräfte für das Institut zu gewinnen. Der Frauenanteil bei Praktika liegt im Schnitt der Jahre 2009 bis 2020 daher bei $36 \%$, was im Vergleich zum branchenüblichen Schnitt von $17 \%$ eine wesentliche Verbesserung darstellt.

\section{Ergebnisse und Wirkungen}

Haupteffekte des FEMtech Karriere-Checks für KMU und des anschließenden FEMtech Karriere Projekts waren die Sensibilisierung und Bewusstseinsbildung aller Mitarbeiterlnnen in Bezug auf Geschlechtergleichheit und -unterschiede. Dank der Projektaktivitäten und der Workshops hat das Thema bei allen MitarbeiterInnen einen höheren Stellenwert bekommen. Auch die Zusammenarbeit im Team hat sich durch das gemeinschaftliche Arbeiten verbessert. Der Freiraum, sich zahlreiche Stunden mit einem neuen, nicht-technischen Thema zu beschäftigen, hat vielmehr wesentlich zum Teambuilding beigetragen.

Ein weiterer nachhaltiger Effekt ergibt sich daraus, dass Gender verstärkt als Querschnittsmaterie in die technischen Forschungsprojekte integriert wurde. Das Gender-Know-how hat sich auch in Hinblick auf weitere Einreichungen für FEMtech Forschungsprojekte und Laura Bassi Ausschreibungen gelohnt. Ohne die Genderkompetenz, die mit Hilfe von FEMtech Karriere aufgebaut werden konnte, hätten die InstitutsmitarbeiterInnen mangels seriösen Nachweises der Kompetenz Abstand von einer Einreichung bei diesen Ausschreibungen genommen. Der Wissenserwerb und das gestärkte Selbstbewusstsein hat eine Teilnahme an diesen Ausschreibungen erst ermöglicht. Auch auf die Projektgestaltung wirkt sich die stärkere Berücksichtigung der Gender- und Diversityperspektive vorteilhaft aus.

Darüber hinaus trägt die Installation der Gender- und Diversitybeauftragten zu einer nachhaltigen Verankerung des Themas bei, da sie trotz harten Wettbewerbes sehr um weitere Aktivitäten in diesem Bereich bemüht ist und den Austausch mit Unternehmen und Netzwerkaktivitäten forciert. PartnerInneninstitute werden beispielsweise im Rahmen von Konferenzen, wie dem neu ins Leben gerufenen Gender-Zirkel des ACR, über die Thematik und mögliche Werkzeuge informiert, um diese auch verstärkt für Gender Mainstreaming Aktivitäten zu gewinnen. 


\section{Lessons learnt und Ausblick}

Die im Rahmen des FEMtech Karriere Projekts entwickelten Produkte und Unterlagen werden weiterhin genutzt und zur Inspiration für weitere Aktivitäten verwendet. Gender Mainstreaming ist im Institut und bei den Forschungsprojekten nachhaltig verankert. Ein weiteres FEMtech KarriereProjekt mit dem Fokus, die Themen Gender und Work-Life-Balance am Institut noch stärker voranzutreiben ist denkbar.

\subsection{4 | Fallstudie im-plan-tat Raumplanungs-GmbH \& Co KG}

\section{Organisation}

Die im-plan-tat Raumplanungs GmbH \& Co KG, die in Niederösterreich angesiedelt ist, widmet sich der Mobilitäts- und Regionalplanung. Im Detail bietet sie Planungen und Beratungen im Bereich der Elektromobilität, der Tourismus- und Freizeitinfrastruktur, GIS-gestützte räumliche Analysen, Stadt- und Ortsplanungen an und unterstützt Unternehmen beim Ressourcen-, Umweltund Fuhrparkmanagement.

Das Planungsbüro im-plan-tat wurde 2002 von vier Gesellschafterlnnen gegründet, die weiterhin als GeschäftsführerInnen im Unternehmen tätig sind. Darüber hinaus arbeiten per Stand Oktober 20205 MitarbeiterInnen im Unternehmen. Insgesamt liegt der Frauenanteil bei 56 \%. Im Jahr 2020 ist das Team etwas geschrumpft, es wird aber schon daran gearbeitet, die Belegschaft wieder aufzustocken. Im Oktober 2020 wurde bereits eine neue Mitarbeiterin aufgenommen und für 2021 ist weiterer Personalzuwachs geplant. Dabei wird besonders auf das gute Arbeitsklima im Unternehmen hingewiesen und auf die persönliche Situation der Bewerberlnnen eingegangen. Die Flexibilität und die Rücksichtnahme auf individuelle Bedürfnisse aufgrund von Kinderbetreuung, Weiterbildung und Pflege werden als essentielle Teile der Unternehmenskultur beschrieben. Gerade in Zusammenhang mit den Maßnahmen zur Eindämmung der Corona-Pandemie erweisen sich flexible Arbeitszeiten als besonders wichtig, aber auch digitale Kommunikationsmittel kommen verstärkt zum Einsatz. Regelmäßige Arbeitstreffen finden jedoch unter Einhaltung entsprechender Schutzmaßnahmen weiterhin statt, um auch einen direkten, persönlichen Austausch zu ermöglichen.

\section{Ziele und Motivation}

Das Unternehmen hat sich bereits im Jahr 2011 an einem FEMtech Karriere Projekt beteiligt, da es bewusst junge, engagierte Frauen fördern wollte. Das Projekt namens „WoGoTo - Women going to the top“, zielte darauf ab, das Team für das Thema Gender zu sensibilisieren, die Mitarbeiterinnen im männlich dominierten Berufsumfeld zu stärken, eine Teamleiterin zu etablieren und eine bessere Work-Life-Balance zu ermöglichen. Durch die Projektaktivitäten ist es u. a. gelungen, eine Mitarbeiterin als Teamleiterin aufzubauen. Das Folgeprojekt im Jahr 2015 namens „WoSuTo - Women succeeding at the top“ zielte darauf aufbauend ab, Frauen dabei zu unterstützen, sich in führenden Positionen langfristig halten zu können und keine karrieretechnischen Einbußen durch Karenzzeiten und Kinderbetreuungspflichten hinnehmen zu müssen. In diesem Zusammenhang wurde auch die nachhaltige Weiterentwicklung zu einem langfristig stabilen, gemischten Team - unter Beibehaltung des branchenuntypisch hohen Frauenanteils - angestrebt. 


\section{Projektinhalte}

Das erste FEMtech Karriere Projekt „WoGoTo - Women going to the top“ wurde von 03/2012 bis 03/ 2013 umgesetzt. Im Rahmen von Reflexionsworkshops und Coaching-Aktivitäten erfolgte der Aufbau einer Mitarbeiterin als Teamleiterin und stellvertretenden Geschäftsführerin. Außerdem wurde ein sehr flexibles Arbeitszeitmodell installiert, das gesamte Team hinsichtlich der Genderthematik sensibilisiert und in ihrer Zusammengehörigkeit gestärkt. Die Mitarbeiterinnen konnten auch ihr Selbstbewusstsein in ihrer Rolle als Technikerinnen steigern.

Darauf aufbauend wurde im zweiten FEMtech Karriere Projekt „WoSuTo-Women succeeding at the top“ im Zeitraum von 07/2015 bis 12/2016 an der nachhaltigen Verankerung der Genderkompetenz im Team gearbeitet und eine Mitarbeiterin zu einer internen Genderbeauftragten ausgebildet. Im Rahmen eines gendersensiblen Stimmtrainings wurden die Mitarbeiterinnen gecoacht, wie sie mit einem positiven Einsatz ihrer Stimme ihre Präsenz und ihr Durchsetzungsvermögen in der männlich dominierten Branche noch weiter erhöhen können. Gerade in ihrem Betätigungsfeld mit vielen männlichen EntscheidungsträgerInnen ist es für die Frauen immer noch schwierig, sich zu behaupten und ihre Kompetenz unter Beweis zu stellen. Weitere Themenschwerpunkte stellten die Verbesserung der Vereinbarung von Familie und Beruf bzw. von Weiterbildung und Beruf dar. Im Rahmen von verschiedenen Workshops wurden Maßnahmen und Tools zur Optimierung der Kommunikationsflüsse erarbeitet, damit die aus der Karenz zurückgekehrten MitarbeiterInnen trotz Stundenreduktion als ProjektleiterInnen weiterarbeiten und ihre Positionen als Team- oder Projektleiterlnnen festigen können.

\section{Ergebnisse und Wirkungen}

Die Hauptziele der beiden FEMtech-Karriere Projekte, nämlich einerseits verstärkt Frauen für Leitungsfunktionen zu gewinnen und diese andererseits trotz Auszeiten für Familie und Weiterbildung langfristig zu erhalten, wurden erreicht. Beim im-plan-tat Raumplanungsbüro hat sich ein Team mit einem hohen Frauenanteil, auch in den Leitungspositionen etabliert. Um dies zu ermöglichen, wurde ein System der Zusammenarbeit in Zweierteams installiert. Dieses ermöglichte flexibel auf die veränderten Lebensrealitäten der MitarbeiterInnen zu reagieren und hat sich beim regen Wechsel im Team aufgrund von Karenzen bewährt.

Um den Arbeitsalltag aller MitarbeiterInnen zu erleichtern und die Abstimmung der Tätigkeiten trotz sich verändernder Teamzusammensetzungen und Arbeitsmodelle möglichst effizient zu gestalten, wurden verschiedene neue Kommunikationsinstrumente eingeführt. Ein Beispiel dafür ist das Instrument der „Stehung“, d. h. im zweiwöchigen Rhythmus steht das Team für 15 Minuten zusammen und hält auf einem Whiteboard die aktuellen Projekte inklusive der in den folgenden zwei Wochen zu erledigenden Arbeiten und Zuständigkeiten fest. Eine weitere neue Maßnahme, die eingeführt wurde, ist der „Kommunikationsraum“, welcher für Vier-Augen-Gespräche auf neutralem Boden genutzt werden kann. Auch die Jour-fix Termine wurden durch Einführung eines "Quick-Checks“ zur Festlegung der Gesprächsinhalte effizienter gestaltet. Darüber hinaus wurden die regelmäßigen MitarbeiterInnengespräche wieder aufgenommen und Leitfäden dafür entwickelt. Als weitere Kommunikationsformate waren Jahresworkshops und Kreativtreffs geplant. 


\section{Lessons learnt und Ausblick}

Die FEMtech Karriere-Projekte bewirkten eine stärkere Implementierung des Gender Mainstreamings sowohl innerhalb des Unternehmens als auch beim Auftritt nach außen. Das Thema ist innerhalb des Teams auf großes Interesse gestoßen, daher werden weiterhin regelmäßige Briefings und Informationen zum Thema in die regelmäßigen Jour fix-Termine eingebaut. An der Erhaltung der eingeführten Kommunikationsformen und der guten Gesprächskultur wird kontinuierlich weitergearbeitet. Auch in Zusammenhang mit den erforderlichen Maßnahmen zur Eindämmung der Corona-Pandemie war der regelmäßige Teamaustausch sehr hilfreich.

Insbesondere aber kommt Gender Mainstreaming verstärkt in der Außenkommunikation, d. h. bei neuen Projekten und der Kommunikation mit Kundlnnen und GeschäftspartnerInnen zum Tragen. Das betrifft jedoch nicht nur eine gendergerechte Sprechweise - gerade deren Einsatz ist oft behutsam zu wählen, um männliche EntscheidungsträgerInnen in der Branche nicht vor den Kopf zu stoßen, sondern in erster Linie, um die Anliegen von Frauen und Familien in planerische Entscheidungen miteinzubringen. Dafür war auch hilfreich, dass die Mitarbeiterinnen durch die Workshop- und Coachingaktivitäten gestärkt wurden und sie lernten, sich in männlichen Entscheidungsgremien besser zu behaupten. Für die Platzierung von Genderthemen in der planerischen Arbeitspraxis ist es wichtig, eine gewisse Sensibilität mitzubringen und diese über die fachliche Ebene zu platzieren.

Mit Hilfe der FEMtech Karriere Projekte konnten die MitarbeiterInnen bei der Bewältigung der Phase der gleichzeitigen Familiengründung und Karriereentwicklung unterstützt werden. Insbesondere den Frauen im Team wurden nach der Rückkehr aus Karenzzeiten ermöglicht, ihre bisherigen Positionen einzunehmen und die Entwicklung ihrer Karriere weiter voranzutreiben. Auch in Zukunft stellen private Auszeiten und zeitliche Einschränkungen der Arbeitszeit kein Hindernis dar, Leitungsfunktionen im Unternehmen zu übernehmen.

Das im-plan-tat Planungsbüro arbeitet an diesen Themen weiter, wobei vor allem auf die Erhaltung der guten Gesprächskultur und der flexiblen Abstimmung des Teams Wert gelegt wird. Aber auch das Thema Gesundheit wird verstärkt aufgegriffen. Weiterer Handlungsbedarf wird in Hinblick auf den gendergerechten Auftritt des Unternehmens gesehen. Die Sensibilität für die Geschlechtergerechtigkeit ist im Unternehmen vorhanden, manchmal fehlen jedoch im regulären Arbeitsalltag die Zeitressourcen, um weitere Aktivitäten umzusetzen. Im Moment wird aktuell kein Bedarf für ein weiteres FEMtech Karriere Projekt gesehen. Es wird aber nicht ausgeschlossen, dass ein solches in Zukunft wieder einmal von Interesse sein könnte. Die Projekte werden als besonders hilfreich wahrgenommen, weil sie den Unternehmen den Rahmen und die Struktur geben, sich mit dem Thema Chancengleichheit auseinanderzusetzen. 


\subsection{5 | Fallstudie - NOUS Wissensmanagement GmbH}

\section{Zur Organisation}

Die NOUS Wissensmanagement GmbH ist im Bereich App Development, Mobile Guides und Projekten der digitalen Transformation tätig. Das Unternehmen wurde 2006 gegründet und verfügt neben dem Standort in Wien mittlerweile über zwei internationale Niederlassungen in den USA und den Vereinigten Arabischen Emiraten. Als Organisationsform liegt eine Wabenstruktur vor, d. h. es gibt vier Teams, die in sehr flachen Hierarchien zusammenarbeiten. Außer dem Team Office mit den Bereichen Geschäftsführung, Human Resources, Buchhaltung und Finanzen bestehen die übrigen Teams jeweils aus Projektmanagement und Softwareentwicklung.

Bei NOUS sind 202035 MitarbeiterInnen beschäftigt, der Frauenanteil liegt bei $30 \%$. Vor allem über die Einstellung von Praktikantinnen gelingt es immer wieder, Mitarbeiterinnen für das Unternehmen zu gewinnen. Auch der Anteil der Teilzeitbeschäftigten ist bei NOUS mit $75 \%$ bei den Frauen und $57 \%$ bei den Männern sehr hoch. Das Team ist sehr jung, innovativ und offen. Viele Arbeitskräfte widmen sich neben der Arbeit noch Betreuungspflichten oder einem Studium und können ihre Arbeitsaufgaben zeitlich sehr flexibel erledigen. Das Unternehmen ist laufend bemüht, neue Modelle zu erproben, um die Arbeit noch flexibler zu machen.

\section{Ziele und Motivation}

NOUS wurde durch Mundpropaganda aus ihrem Netzwerk auf die Förderung im Rahmen von FEMtech Karriere aufmerksam. Aufgrund von Empfehlungen sich auch in diesem Bereich stärker zu engagieren, wurde der FEMtech Karriere-Check für KMU in Angriff genommen. Dieses „genderfair"-Projekt diente einer Bestandsaufnahme mit Maßnahmenableitung mit dem langfristigen Ziel, mehr Mädchen und Frauen in technischen Berufsfeldern in das Unternehmen zu bringen. Darauf aufbauend wurde das FEMtech Karriere Projekt „NOUS FEMomenal“ realisiert, das auch das Ziel verfolgte, den Anteil der Mitarbeiterinnen zu erhöhen. Zudem sollte das Bewusstsein für die Gleichstellung im Unternehmen verankert und standardisiert werden. Zudem erhoffte man sich durch die gelebte Gleichstellung im Unternehmen im Wettbewerb um potenzielle MitarbeiterInnen Vorteile. Außerdem sollte die zeitgerechte Fertigstellung von Projekten durch ein zu entwickelndes innovatives Bonus-Zeit-Modell gefördert werden.

\section{Projektinhalte}

Die NOUS Wissensmanagement $\mathrm{GmbH}$ führte im Zeitraum von 06/2018 bis 05/2019 einen FEMtech Karriere-Check für KMU namens "genderfair" durch. Dabei erfolgte eine umfassende Genderanalyse mit Hilfe der 4-R Methode, bei der Repräsentation, Ressourcen, Realitäten sowie Rechte/Regelungen im Unternehmen umfassend analysiert und darauf aufbauend Maßnahmen abgeleitet wurden. Handlungsbedarf wurde im Bereich des Recruiting hinsichtlich der Transparenz sowie im Bereich der Vereinbarkeit von Familie und Beruf von Seiten der MitarbeiterInnen geortet. Aus Sicht des Unternehmens wurde die zeitgerechte Fertigstellung von Softwareprojekten als problematisch wahrgenommen, die auf die mangelnde Flexibilität der Teilzeitkräfte zurückgeführt wurde. 
Diese Themenkreise wurden im Rahmen des nachfolgenden FEMtech Karriere Projekts „NOUS FEMomenal" im Zeitraum von 04/2019 bis 12/2020 aufgegriffen und weiterbearbeitet. Die ursprüngliche Projektlaufzeit, die bis 06/2020 vorgesehen war, wurde aufgrund der Corona-Pandemie um 6 Monate verlängert. Den Schwerpunkt des Projekts sollte die Entwicklung eines innovativen Bonus-Zeit-Modell bilden, um die Zeitproblematik bei der Projektfertigstellung zu entschärfen. Es war angedacht, Teilzeit-MitarbeiterInnen über ein Bonussystem zu motivieren, zeitweise mehr zu arbeiten, um Projekte besser rechtzeitig fertigstellen zu können. Eine genaue Analyse der Arbeitssituation und eine Befragung der Mitarbeiterlnnen ergab jedoch, dass die Probleme bei der Fertigstellung nicht auf Vollzeit- oder Teilzeitanstellungen zurückzuführen sind, sondern von vielen individuelleren Faktoren abhängig sind.

Daher wurde nun versucht, die Prozesse und Strukturen für alle Mitarbeiterlnnen unabhängig von einer Vollzeit- oder Teilzeitanstellungen zu optimieren. Dazu wurde ein Modell für flexibles Arbeiten entwickelt, das genau definiert, wie die Kommunikationsprozesse ablaufen müssen, um diese Flexibilität zu ermöglichen. In diesem Zusammenhang wurden auch die Rollen und Verantwortlichkeiten der MitarbeiterInnen genauer definiert, das Thema der Verantwortungsübernahme im Rahmen eines Workshops thematisiert und schließlich den einzelnen Arbeitskräften mehr Verantwortung übertragen.

Auch das Thema Home-Office wurde schon vor der Ausbreitung des Corona-Virus in den Arbeitsalltag von NOUS integriert. Dazu wurde die Möglichkeit geschaffen, dass Meetings nicht persönlich, sondern auch online besucht werden können. Die Besprechungen werden generell ausschließlich am Vormittag abgehalten, um Personen mit Betreuungspflichten eine ungehinderte Teilnahme zu ermöglichen. Die Kommunikationsrichtlinien wurden entsprechend erweitert und für die Zeit nach der Corona-Pandemie wurde ein Home-Office Modell entwickelt, das für jeden/e Mitarbeiterln ein gewisses, frei einteilbares Kontingent an Home-Office Tagen vorsieht.

Darüber hinaus wurden zum Aufbau von Genderkompetenz Workshops durchgeführt und individuelles Coaching und Mentoring für die MitarbeiterInnen angeboten. Das Coaching ermöglichte den MitarbeiterInnen, für sie persönlich relevante Themen aufzugreifen. Darüber hinaus fanden auch Gruppenveranstaltungen zu Themen wie Teamleitung oder den Umgang mit Konflikten statt.

Um verstärkt Frauen als Mitarbeiterinnen zu gewinnen, möchte NOUS vor allem mit den flexiblen Arbeitsmöglichkeiten punkten und diese gezielt bewerben. Dazu wird ein ansprechendes Printmaterial erstellt, das auf Karrieremessen oder in Shared Offices zur MitarbeiterInnensuche zum Einsatz kommt. Auch im Bereich der Öffentlichkeitsarbeit werden noch Artikel erstellt und auf Websites Informationen zu den Themen Frauen in der Technik, Work-Life Balance und flexibles Arbeiten verbreitet.

\section{Ergebnisse und Wirkungen}

NOUS hat flexibles Arbeiten und die Möglichkeiten der Online-Kommunikation und des HomeOffice durch die Entwicklung von Prozess- und Kommunikationsmodellen standardmäßig in den Arbeitsalltag integriert. Da sich das Unternehmen so strukturiert und umfassend mit diesen Arbeitsweisen auseinandergesetzt hat, war es im Zuge der Corona-Pandemie bereits gut darauf 
vorbereitet. Das flexible Arbeitsangebot kommt vor allem Frauen entgegen. Daher ist geplant, dieses in Zukunft noch stärker zu bewerben, um weitere weibliche Arbeitskräfte für das Unternehmen zu gewinnen.

Der Frauenanteil im Unternehmen hat sich im Zuge der Projektaktivitäten bereits leicht erhöht. Im Rahmen des Karriere-Checks für KMU wurde ein Frauenanteil von $26 \%$ erhoben, mittlerweile liegt dieser bei $30 \%$. Im Unternehmen sind auch einige weibliche IT-Fachkräfte tätig.

Die bereits beschäftigten MitarbeiterInnen konnten vor allem von den Coaching-, Mentoring- und Schulungsangeboten profitieren. Ihre Rollen und Verantwortlichkeiten in der Projektarbeit wurden geschärft und damit die Organisationsstruktur noch weiter optimiert.

\section{Lessons learnt und Ausblick}

Die implementierten Prozess- und Kommunikationsrichtlinien, die genau definiert und festgelegt wurden, sichern die nachhaltige Umsetzung der flexiblen Arbeitsweisen. Durch die Projektaktivitäten ist flexibles Arbeiten ganz normal geworden. Auch bei der Geschäftsführung ist die Akzeptanz dafür maßgeblich gestiegen. Das Übertragen von mehr Verantwortung an die MitarbeiterInnen wirkte sich ebenfalls positiv auf die Projektarbeit aus.

In Zukunft werden weiterhin Bemühungen gesetzt, um mehr weibliche Arbeitskräfte mit Hilfe der flexiblen Arbeitsangebote zu gewinnen. Auch die Durchführung eines weiteren FEMtech Karriere Projekts wäre vorstellbar. Vorteil dieser Förderung ist, dass sie eine gewisse Verpflichtung von der Geschäftsführung erfordert, sich intensiver diesem Thema anzunehmen. Auch die Schwerpunktsetzung auf das Thema der Gleichberechtigung anstelle der ausschließlichen Frauenförderung wird vorteilhaft wahrgenommen, da so eine breitere Themenpalette aufgegriffen werden kann.

\subsection{6 | Fallstudie - RISC Software GmbH}

\section{Zur Organisation}

Die RISC Software GmbH ist ein national und international tätiges Forschungs- und Entwicklungsunternehmen, das zu $80 \%$ im Eigentum der Johannes Kepler Universität Linz sowie zu $20 \%$ im Eigentum der Upper Austrian Research $\mathrm{GmbH}$ des Landes Oberösterreich steht. Die RISC Software GmbH wurde als anwendungsorientierter Teil des RISC Instituts im Jahr 1992 gegründet. Insgesamt ist das Unternehmen von einer Dualität von Grundlagenforschung (RISC Institut) und Anwendung (RISC Software $\mathrm{GmbH}$ ) geprägt, wobei die Entwicklung individueller Softwarelösungen für Unternehmen, Medizin und Industrie im Vordergrund steht. Im Unternehmen sind 56 Männer und 23 Frauen tätig, die Frauenquote liegt damit bei $30 \%$.

Die Rahmenbedingungen und Arbeitszeiten im Unternehmen werden von den Mitarbeiterlnnen sehr geschätzt, da sie ganz flexibel ohne jegliche Kernzeiten arbeiten können. Auch verschiedene Teilzeitmodelle, die von Frauen wie Männern in Anspruch genommen werden, und Home-OfficeMöglichkeiten werden angeboten. 


\section{Ziele und Motivation}

Die Geschäftsführung der RISC Software GmbH ist seit Jahren bemüht, gezielt Frauen in der Technik zu unterstützen und die Gleichstellung der MitarbeiterInnen im Unternehmen zu fördern. Um den Frauenanteil im Unternehmen langfristig zu erhöhen und sich verstärkt als attraktive/r Arbeitgeberln für Frauen in der Technik zu positionieren, wurde bereits im Jahr 2009 die FEMtech Karriere Förderung aufgegriffen. Das Projekt FUTURISC, das im Zeitraum von 03/2011 bis 10/2012 umgesetzt wurde, half bei der Vermittlung von Genderwissen und unterstützte die Aufnahme von weiblichen Mitarbeiterinnen. Auch in den folgenden Jahren wurden verstärkt Frauen als Technikerinnen und Forscherinnen beschäftigt. Darauf aufbauend zielte das FEMtech Karriere Projekt FemPowerED@RISC im Jahr 2016 darauf ab, das vorhandene Potential der Mitarbeiterinnen zu stärken und sie auf die Tätigkeit als Projektleiterinnen vorzubereiten. Zusätzlich galt es, die Außenwahrnehmung des Unternehmens zu optimieren, die Förderung von Frauen für Außenstehende sichtbar zu machen und den Einstieg neuer MitarbeiterInnen in das Unternehmen zu erleichtern.

\section{Projektinhalte}

Das FEMtech Karriere Projekt FemPowerED@RISC, das im Zeitraum von 10/2017 bis 09/2018 abgewickelt wurde, unterstützte gezielt Frauen in der Technik. Um Genderkompetenz im Unternehmen aufzubauen, wurden Workshops zu Gender Mainstreaming, gendersensibler Kommunikation und zur Auseinandersetzung mit dem Thema Frauen in der Technik veranstaltet. Darüber hinaus fanden verschiedene Weiterbildungsmaßnahmen statt, um ausgewählte Mitarbeiterinnen auf Führungsrollen im Unternehmen vorzubereiten. Vor allem Mitarbeiterinnen aus dem Bereich Softwareentwicklung sollten nach Ende des Projekts schrittweise Projektleitungsfunktionen übernehmen. Dazu wurden externe und interne Trainings zu Führungskompetenzen, Konfliktmanagement, F\&E-Antragsabwicklung, Projektmanagement und (Projekt-)Controlling abgehalten. Die Workshops empfanden die Teilnehmerinnen als sehr hilfreich, wobei das Führungskräftetraining als besonderes Highlight beschrieben wurde, da es neben der Vorbereitung auf eine Führungsrolle auch viel Inspiration für die Persönlichkeitsentwicklung gab. Die angehenden Projektleiterinnen konnten Kommunikations- und Konfliktlösetechniken erlernen, die heute noch zur praktischen Anwendung kommen.

Im Bereich der Öffentlichkeitsarbeit und zur Entwicklung konkreter Rollenvorbilder für Frauen in Technik, wurden schriftliche und/oder Video-Interviews mit Mitarbeiterinnen durchgeführt und über diverse Kanäle (Webseite, Social Media, Presse) veröffentlicht. Es erfolgte die Entwicklung einer speziellen Landingpage auf der Webseite, welche die Maßnahmen zur Frauenförderung innerhalb der RISC Software GmbH beschreibt und somit besonders Frauen ermutigt, sich zu bewerben. Auch Vorträge an Bildungseinrichtungen wurden abgehalten, um die Vorbildwirkung zu verstärken und gezielt Frauen anzusprechen.

Um neuen MitarbeiterInnen den Einstieg in die RISC Software GmbH zu erleichtern, wurde ein Mentoring-Curriculum für diese Zielgruppe entwickelt. Neue MitarbeiterInnen erhalten seither eine MitarbeiterInnenmappe mit einer Übersicht der wichtigsten internen Prozesse und werden in den ersten Wochen von organisatorischen und fachlichen MentorInnen betreut und über alle Aufgaben und Zuständigkeiten informiert. Bei der Entwicklung des Mentoring-Konzepts wurden 
auch die Berufsbezeichnungen überarbeitet und Checklisten mit allen Schritten für einen systematischen Personaleintritt erstellt. Das Mentoring hat bei den neuen MitarbeiterInnen großen Anklang gefunden und soll daher noch weiterentwickelt werden.

\section{Ergebnisse und Wirkungen}

Der RISC Software GmbH war es wichtig, die Gender-Maßnahmen unternehmensweit zu verankern und das Genderbewusstsein bei allen Mitarbeiterlnnen aufzubauen und nicht einzelne Gendervertrauenspersonen in diesem Bereich zu qualifizieren. Das Gender-Thema soll somit durch MitarbeiterInnen aus möglichst vielen organisatorischen Bereichen mitgetragen werden. Diese können von vielfältigen neuen Produkten, wie neuen Coaching-Formaten sowie dem Mentoring-Programm für neue MitarbeiterInnen profitieren, die weiterhin laufend zur Anwendung kommen. Auch die Außenwahrnehmung hat sich weiter verbessert, da schon gezielte Anfragen eintrafen, die Aktivitäten des Unternehmens zu präsentieren, um mehr Frauen für die Technik zu gewinnen.

Das FEMtech Karriere Projekt hat außerdem maßgeblich zur Steigerung des Frauenanteils in Führungspositionen beigetragen. Aufgrund der vielfältigen Schulungsaktivitäten haben vier Mitarbeiterinnen erfolgreich den Schritt in das Projektmanagement gewagt und wurden zu Projektleiterinnen befördert. Diese profitierten vor allem von den Weiterbildungsangeboten und machen heute noch von den erlernten Kommunikations- und Projektmanagementtechniken im Arbeitsalltag Gebrauch. Die gezielte Karriereförderung von Frauen und die Ausrichtung der Workshops auf Mitarbeiterinnen führte jedoch auch dazu, dass sich die Männer im Team etwas diskriminiert fühlten.

FemPowerED@RISC intensivierte auch die Vernetzung der Mitarbeiterinnen. Im Zuge des Projekts wurden interne Jour fixes aller Frauen eingeführt. Die firmeninternen Treffen der Mitarbeiterinnen haben das Frauenteam stark zusammengeschweißt. Diese Austauschmöglichkeit alle 23 Monate wird von der Geschäftsleitung unterstützt und weiterhin beibehalten.

\section{Lessons learnt und Ausblick}

Die Förderung von Frauen in der Technik und die langfristige Steigerung des Frauenanteils im Unternehmen bleibt weiterhin auf der Tagesordnung der RISC Software GmbH. Die erfolgreiche Karriereförderung von Frauen wird weiterhin gezielt unterstützt. Dazu wurde in Folge ein transparentes Karriereschema mit den verschiedenen Positionen und Gehaltsstufen erstellt. Auch die kontinuierliche Weiterbildung wird im Rahmen eines eigenen Weiterbildungsprogramms forciert, damit die MitarbeiterInnen immer am neuesten Stand der Technik sind.

Es wird auch überlegt, noch ein weiteres FEMtech Karriere Projekt einzureichen. Ein paar MitarbeiterInnen engagieren sich sehr für dieses Thema und wollen weiterhin in diesem Bereich aktiv bleiben. Bei dem zukünftigen Projekt sollen jedoch Frauen und Männer gleichermaßen angesprochen und eingebunden werden, um keine weitere Diskriminierung zu erzeugen. 


\section{2 | Verzeichnisse}

\section{Grafikverzeichnis}

Grafik 1 | Zufriedenheit der befragten Interview Grants EmpfängerInnen mit der Förderung bzw. Abwicklung der Förderung, in Prozent

Grafik 2 | Zufriedenheit der befragten Relocation Grants EmpfängerInnen mit der Förderung bzw.

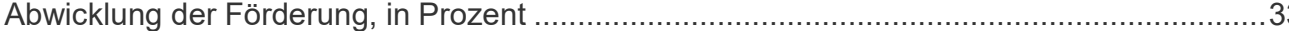

Grafik 3 | Zufriedenheit der befragten Dual Career Grants EmpfängerInnen mit der Förderung bzw.

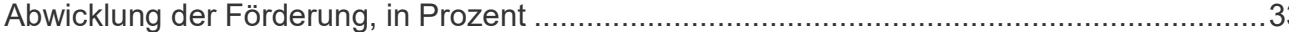

Grafik 4 | Zufriedenheit mit verschiedenen Aspekten der Förderung und Förderabwicklung von FEMtech Karriere und des FEMtech Karriere-Checks für KMU, Anzahl der befragten Organisationen

Grafik 5 | Anzahl der geförderten Praktika für Schülerinnen und Schüler, Ausschreibungen 2011-2019

Grafik 6 | Wissenschaftsdisziplinen und Themenfelder der geförderten Praktika für Schülerinnen und Schüler Ausschreibungen 2011-2019, in Prozent

Grafik 7 | Geförderte Praktika für Schülerinnen und Schüler nach Bundesländern, Ausschreibungen 2011-2019, in Prozent.

Grafik 8 | Praktikumsplätze nach Organisationsarten, Ausschreibungen 2011-2019, in Prozent...............39

Grafik 9 | Anzahl der Praktika pro Organisation im Jahr 2019 und Anteile der Top-10 AnbieterInnen an allen Praktikumsplätzen, Ausschreibungen 2011 - 2019, in Prozent.............................40

Grafik 10 | Praktikumsnutzung in den Schulen: Anteil der involvierten Schulen 2019 und Anteil der SchülerInnen nach der Anzahl der Praktika, Ausschreibungen 2011-2019, in Prozent

Grafik 11 | Nutzung der Praktikumsbörse, Ausschreibungen 2012-2019, in Prozent.

Grafik 12 | PraktikumsteilnehmerInnen nach Geschlecht, Ausschreibungen 2011-2019, Anteile in Prozent...........43

Grafik 13 | Besuchte Schulen der PraktikantInnen, Ausschreibungen 2011-2019, in Prozent.....

Grafik 14 | Anteile der PraktikantInnen aus den verschiedenen Schultypen nach Organisationsarten, Ausschreibungen 2015-2019, in Prozent.

Grafik 15 | Beurteilung des Nutzens der Praktika für SchülerInnen für die Fördernehmerlnnen, Ausschreibungen 2011-2019, in Prozent (Mehrfachnennungen möglich)

Grafik 16 | Beurteilung der Praktika durch die Schülerlnnen, Ausschreibungen 2011-2019, in Prozent

Grafik 17 | Bewertung der Tätigkeiten und Einschätzung des Interesses an naturwissenschaftlichen Berufen durch die Schülerlnnen, Ausschreibungen 2011-2019, in Prozent

Grafik 18 | Beteiligung an den Ausschreibungen 2011-2019, Anzahl der Einreichungen und der

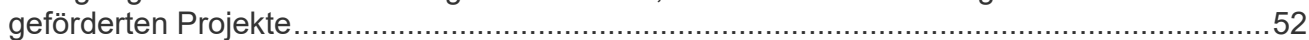

Grafik 19 | Themenfelder der Projekte nach Ausschreibung 2011-2019, in Prozent..............................53

Grafik 20 | Verteilung der FördernehmerInnen, Ausschreibungen 2011-2019, in Prozent..........................55

Grafik 21 | Organisationsform und Bundesland der Konsortialführung, Ausschreibungen 2011-2019, in Prozent

Grafik 22 | Anzahl der Kooperationszuschüsse, Ausschreibungen 2011 - 2016 .................................57

Grafik 23 | Durchschnittliche Anzahl der involvierten Kinder/Jugendlichen pro Projekt, Ausschreibungen 2011-2016

Grafik 24 | Anzahl der geförderten FEMtech Praktika für Studentinnen nach Ausschreibung, 2011-2019

Grafik 25 | Wissenschaftsdisziplinen und Themenfelder der geförderten FEMtech Praktika für Studentinnen, Ausschreibungen 2011-2019, in Prozent. 
Grafik 26 | Dauer der geförderten Praktika, Ausschreibungen 2011-2019, in Prozent

Grafik 27 | Geförderte Praktika für Studentinnen nach Bundesländern, Ausschreibungen 2011-2019, in Prozent

Grafik 28 | Praktika nach Organisationsart, Ausschreibungen 2011-2019, in Prozent....

Grafik 29 | Anzahl der Praktika nach Organisationstyp, Ausschreibungen 2017, 2018 und 2019, in Prozent.

Grafik 30 | Durchschnittliche Semesteranzahl bei den geförderten Praktika, Ausschreibungen 2011-2019

Grafik 31 | Wichtige Informationsquellen über die Möglichkeit eines förderbaren Praktikums für die Praktikantinnen, Ausschreibungen 2011-2018 in Prozent (Mehrfachnennungen möglich).....

Grafik 32 | Beurteilung des Nutzens der Praktika für Studentinnen für die FördernehmerInnen, Ausschreibungen 2011-2018, in Prozent (Mehrfachnennungen möglich).

Grafik 33 | Beurteilung der Praktika durch die Studentinnen, Ausschreibungen 2011-2018, in Prozent.

Grafik 34 | Gründe und Wirkungen der Praktika für die Studentinnen, Ausschreibung 2018 in Prozent (Mehrfachnennungen möglich).

Grafik 35 | Einschätzung des Interesses an einer Tätigkeit in Forschung, Naturwissenschaft und Technik durch die Studentinnen, Ausschreibungen 2011-2018, in Prozent

Grafik 36 | Weiterbeschäftigung der Praktikantinnen, Ausschreibungen 2011-2018, in Prozent .75

Grafik 37 | Hauptzielsetzungen der FEMtech Karriere Projekte, Anzahl der befragten Organisationen (Mehrfachnennungen möglich).

Grafik 38 | Entwicklung der Anzahl der FEMtech Karriere und der FEMtech Karriere-Check Projekte, Ausschreibungen 2011-2019.

Grafik 39 | Anzahl der Art der beteiligten Organisationen und ihrer Projekte, Ausschreibungen 2011-2019

Grafik 40 | Aufmerksamkeit auf die Programmlinie und Initiative zur Teilnahme, Anzahl der befragten Organisationen

Grafik 41 | Durchführung der FEMtech Karriere Projekte und des FEMtech Karriere-Checks für KMU ohne Förderung, Anzahl der befragten Organisationen

Grafik 42 | Herausforderungen bei der Umsetzung der FEMtech Karriere Projekte bzw. des FEMtech Karriere-Checks für KMU, Anzahl der befragten Organisationen.

Grafik 43 | Erzielung folgender Wirkungen durch die FEMtech Karriere Projekte, Anzahl der befragten Organisationen.

Grafik 44 | Steigerung der Akzeptanz und Awareness für das Thema Gleichstellung im Unternehmen, Anzahl der befragten Organisationen....

Grafik 45 | Einsatz von im Rahmen von FEMtech Karriere entwickelten Produkten und Tools, Anzahl der befragten Organisationen.

Grafik 46 | Art der durchgeführten oder geplanten Öffentlichkeitsarbeit im Rahmen von FEMtech Karriere bzw. des FEMtech Karriere-Checks für KMU, Anzahl der befragten Organisationen 93

Grafik 47 | Wichtige Maßnahmen zur Gewinnung von mehr Frauen für Forschung und Technik,

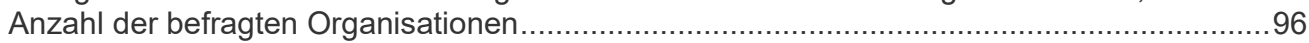

Grafik 48 | Anzahl eingereichter Projekte und Anteil genehmigter Projekte nach Ausschreibungsjahr.......98

Grafik 49 | Anzahl der Beteiligungen an FEMtech Forschungsprojekten nach Organisationstyp, 2011-2018/19

Grafik 50 | Anzahl der Projektbeteiligungen nach Bundesland, 2011-2018 .

Grafik 51 | Anzahl der Projektbeteiligungen pro Organisation, 2011-2018, nach Organisationstyp ..........101

Grafik 52 | Verteilung der geförderten FEMtech Forschungsprojekte nach Themen, 2011-2018 
Grafik 53 | Anteil der erstmals in Talente geförderten Organisationen an allen in FEMtech Forschungsprojekte teilnehmenden Organisationen nach Ausschreibungsjahr, in Prozent

Grafik 54 | Informationsquelle der Geförderten, in Prozent (Mehrfachnennungen möglich)

Grafik 55 | Anzahl Einreichungen und genehmigte Anträge in Karriere-Grants insgesamt, nach Jahr des Eingangsdatums 2011-2020

Grafik 56 | Verteilung des geförderten Karriere Grant Typs und des Geschlechts der EinzelforscherInnen, nach Ausschreibungsjahr 2011-2019, in Prozent

Grafik 57 | Anzahl der Anträge nach Aufenthaltsort der Antragstellerlnnen, nach Kalenderjahr 2012-2019

Grafik 58 | Anzahl der Stellenanbieterlnnen nach Bundesland, 2011-2019....

Grafik 59 | Verteilung der Befragten nach Grant-Art, in Prozent

Grafik 60 | Gründe, warum Befragte keinen Interview Grant vor dem Relocation Grant in Anspruch genommen haben, in Prozent .....

Grafik 61 | Gründe von Interview-Grant Fördernehmerlnnen, warum die angebotene Stelle in Österreich nicht angenommen wurde (Mehrfachantworten möglich)

Grafik 62 | In Anspruch genommene Kosten der Relocation Grants FördernehmerInnen (Mehrfachnennungen möglich)

Grafik 63 | In Anspruch genommene Kosten der Dual Career Grants Fördernehmerlnnen, in Prozent (Mehrfachnennungen möglich)

Grafik 64 | Anteil der Befragten, ob sie alleine, gemeinsam mit dem/der Partnerln und/oder ihren Kindern nach Österreich umgezogen sind, in Prozent (Mehrfachnennungen möglich)

Grafik 65 | Verteilung der Antworten der Relocation Grants FördernehmerInnen auf die Frage, ob ihr/e Partnerln einen Dual Career Grant in Anspruch genommen hat/plant in Anspruch zu nehmen und Gründe falls dies nicht der Fall war/ist, in Prozent....

Grafik 66 | Wirkungen des Interview Grants auf die Entscheidung, für ein Job Interview nach Österreich zu kommen, in Prozent

Grafik 67 | Wirkungen des Relocation Grants auf die Entscheidung, aus beruflichen Gründen nach Österreich umzuziehen, in Prozent.

Grafik 68 | Weiterempfehlung von Karriere Grants an Kolleginnen und Kollegen, in Prozent.

Grafik 69 | Entwicklung der Anzahl veröffentlichter Jobs, der Websiteaufrufe (page impressions) und der WebsitebesucherInnen (unique visitors) der FFG Jobbörse, 2013-2019.....

Grafik 70 | Verteilung der Art der Veröffentlichungen in der Jobbörse, nach Quartal, 3. Quartal 2014 bis 4. Quartal 2019

Grafik 71 | Anzahl der Veröffentlichungen in der Jobbörse nach Organisationstyp, 01.07.2019 bis 24.06.2020

Grafik 72 | Verteilung der Veröffentlichungen in der Jobbörse nach Wissenschaftsdisziplin, in Prozent der Stellenausschreibungen

Grafik 73 | Verteilung der geförderten Organisationen/Einzelforscherlnnen und Projektbeteiligungen in Talente, nach Organisationstyp in Prozent

Grafik 74 | Verteilung der Summe der genehmigten Förderbarwerte nach Programmlinie, in Prozent des Gesamtbarwerts, 2011-2019.

Grafik 75 | Verteilung der erstmals geförderten Organisationen in Talente (ohne Karriere-Grants) sowie der Organisationen die erstmals durch ihre Teilnahme an Talente von der FFG gefördert wurden, nach Talente-Programmlinien, in Prozent.....

Grafik 76 | Verteilung der geförderten Organisationen nach ihrer Teilnahme in unterschiedlichen Talente (ohne Karriere-Grants) Programmlinien*, in Prozent.

Grafik 77 | Überblick über andere von Talente-Teilnehmern in Anspruch genommene Förderungen aus den FFG Programmen, in Prozent. 


\section{Tabellenverzeichnis}

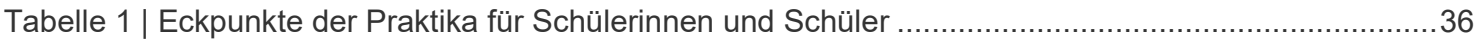

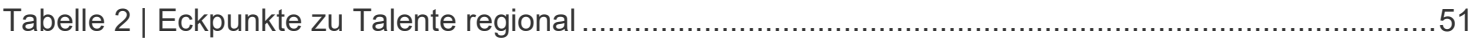

Tabelle 3 | Eckpunkte zu FEMtech Praktika für Studentinnen ..........................................................63

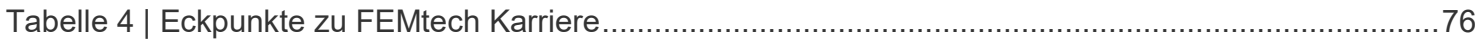

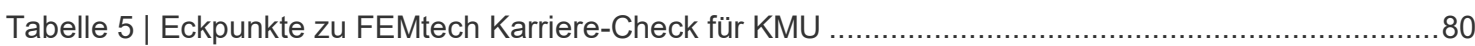

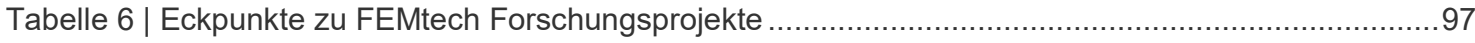

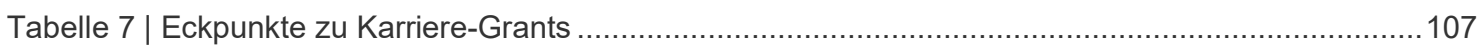

Tabelle 8 | Zielerreichung Interventionsfeld Talente entdecken - Nachwuchs ...................................136

Tabelle 9 | Zielerreichung Interventionsfeld Talente nützen - Chancengleichheit ................................138

Tabelle 10 | Zielerreichung Interventionsfeld Talente nützen - Forscherinnen und Forscher ..................140

\section{Literaturverzeichnis}

European Commision (2019): She Figures 2018

Gruber, Benjamin / Schmid, Kurt (2018): Review Talente regional, Endbericht. Institut für Bildungsforschung der Wirtschaft (ibw)

Heckl, E. / Wolf, Laurenz (2014): Zwischenevaluierung des Förderschwerpunkts Talente, Endbericht. KMU Forschung Austria

Nindl, E. / Kaufmann, P. (2020): Wirkungsmonitoring der FFG Förderungen 2019, Endbericht. KMU Forschung Austria

Wroblewski, A. (2016): Gender in Forschungsinhalten. Review der FEMtech Forschungsprojekte 2008 bis 2014. Institut für Höhere Studien (IHS) 

KMU Forschung Austria

Austrian Institute for SME Research

www.kmuforschung.ac.at 